\title{
Diophantine approximation as Cosmic Censor for Kerr-AdS black holes
}

\author{
Christoph Kehle ${ }^{1,2}$
}

Received: 2 August 2020 / Accepted: 24 September 2021 /

Published online: 24 November 2021

(C) The Author(s) 2021

\begin{abstract}
The purpose of this paper is to show an unexpected connection between Diophantine approximation and the behavior of waves on black hole interiors with negative cosmological constant $\Lambda<0$ and explore the consequences of this for the Strong Cosmic Censorship conjecture in general relativity. We study linear scalar perturbations $\psi$ of Kerr-AdS solving $\square_{g} \psi-\frac{2}{3} \Lambda \psi=0$ with reflecting boundary conditions imposed at infinity. Understanding the behavior of $\psi$ at the Cauchy horizon corresponds to a linear analog of the problem of Strong Cosmic Censorship. Our main result shows that if the dimensionless black hole parameters mass $\mathfrak{m}=M \sqrt{-\Lambda}$ and angular momentum $\mathfrak{a}=a \sqrt{-\Lambda}$ satisfy a certain non-Diophantine condition, then perturbations $\psi$ arising from generic smooth initial data blow up $|\psi| \rightarrow+\infty$ at the Cauchy horizon. The proof crucially relies on a novel resonance phenomenon between stable trapping on the black hole exterior and the poles of the interior scattering operator that gives rise to a small divisors problem. Our result is in stark contrast to the result on Reissner-Nordström-AdS (Kehle in Commun Math Phys 376(1):145-200, 2020) as well as to previous work on the analogous problem for $\Lambda \geq 0$-in both cases such linear scalar perturbations were shown to remain bounded. As a result of the non-Diophantine condition,
\end{abstract}

Christoph Kehle

christoph.kehle@eth-its.ethz.ch

1 Department of Pure Mathematics and Mathematical Statistics, University of Cambridge, Wilberforce Road, Cambridge CB3 0WB, UK

2 Institute for Theoretical Studies, ETH Zürich, Clausiusstrasse 47, 8092 Zürich, Switzerland 
the set of parameters $\mathfrak{m}, \mathfrak{a}$ for which we show blow-up forms a Baire-generic but Lebesgue-exceptional subset of all parameters below the Hawking-Reall bound. On the other hand, we conjecture that for a set of parameters $\mathfrak{m}, \mathfrak{a}$ which is Baire-exceptional but Lebesgue-generic, all linear scalar perturbations remain bounded at the Cauchy horizon $|\psi| \leq C$. This suggests that the validity of the $C^{0}$-formulation of Strong Cosmic Censorship for $\Lambda<0$ may change in a spectacular way according to the notion of genericity imposed.

\section{Contents}

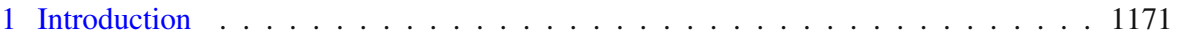

Outline of the introduction . . . . . . . . . . . . . . . . . . . . 1173

1.1 Strong Cosmic Censorship: Conjectures 1-4 . . . . . . . . . . . . . . . . . 1173

1.2 Exterior: log-decay, quasi(normal) modes and semi-classical analysis . . . . . 1182

1.3 Interior: scattering from event to Cauchy horizon . . . . . . . . . . . . . 1186

1.4 Small divisors and relation to Diophantine approximation . . . . . . . . . . . . 1187

1.5 Conjecture 5 and Conjecture 6 replace Conjecture 3 and Conjecture 4 for Kerr-AdS 1191

1.6 Theorem 1: Conjecture 5 is true . . . . . . . . . . . . . . . . . . . . . . . . . 1194

1.7 Outlook on Conjecture 6 . . . . . . . . . . . . . . . . . . . . . . . . . . 1194

1.8 Turning the heuristics of Section 1.4 into a proof of Theorem 1 . . . . . . . . . 1194

1.9 Outline of the paper . . . . . . . . . . . . . . . . . . . . . . . . . 1199

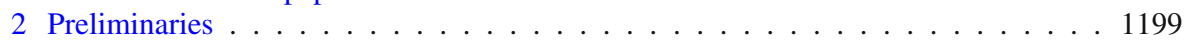

2.1 Fractal measures and dimensions . . . . . . . . . . . . . . . . . . . . . . . . 1199

2.2 Kerr-AdS spacetime . . . . . . . . . . . . . . . . . . . . . . . 1201

2.3 Conventions . . . . . . . . . . . . . . . . . . . . . . . . 1205

2.4 Norms and energies . . . . . . . . . . . . . . . . . . . . 1206

2.5 Well-posedness and log-decay on the exterior region . . . . . . . . . . . . . 1207

2.6 Separation of variables: radial o.d.e., angular o.d.e. and coupling constants $\lambda_{m \ell}(a \omega) \quad 1210$

3 The angular o.d.e. . . . . . . . . . . . . . . . . . . . 1211

3.1 Angular potential $W_{1}$ at interior scattering poles in semi-classical limit . . . . 1212

3.2 Angular eigenvalues at interior scattering poles with $\lambda_{m_{i} \ell_{i}}=\tilde{\lambda}_{0} m_{i}^{2}+O(1) \quad \ldots 1215$

3.3 Bounds on $\partial_{\xi} \lambda_{m \ell}$ and $\partial_{\xi} S_{m \ell}$ near interior scattering poles . . . . . . . . . . 1219

3.4 Semi-classical resolvent estimates near interior scattering poles . . . . . . . . . 1225

4 The radial o.d.e. on the exterior . . . . . . . . . . . . . . . . . . 1245

4.1 Resonance: Radial o.d.e. at interior scattering poles allows for stable trapping . 1245

4.2 Fundamental pairs of solutions associated to trapping . . . . . . . . . . . 1249

5 The non-Diophantine condition . . . . . . . . . . . . . 1256

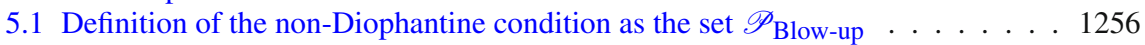

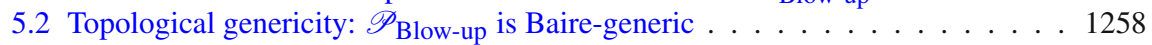

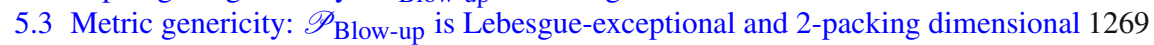

6 Construction of the initial data . . . . . . . . . . . . . . . . . . . 1270

7 Exterior analysis: from the initial data to the event horizon . . . . . . . . . . . . 1275

7.1 Cut-off in time and inhomogeneous equation . . . . . . . . . . . . . . . . . 1275

7.2 Estimates for the inhomogeneous radial o.d.e. . . . . . . . . . . . . . . . . 1277

7.3 Representation formula for $\psi$ at the event horizon . . . . . . . . . . . . . . 1283

8 Interior analysis: Estimates on radial o.d.e. and interior scattering poles . . . . . . . 1285

8.1 Radial o.d.e. on the interior: fixed frequency scattering . . . . . . . . . . . . 1285

8.2 Scattering poles: representation formula for $\psi$ on the interior . . . . . . . . . . 1298

9 Proof of Theorem 1: Small divisors lead to blow-up . . . . . . . . . . . . . 1301

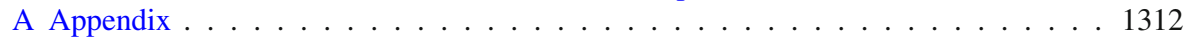


A.1 Airy functions . . . . . . . . . . . . . . . . . . . . . . . . . 1312

A.2 Parabolic cylinder functions . . . . . . . . . . . . . . . . . . . . . . . . . 1314

References . . . . . . . . . . . . . . . . . . . . . 1316

\section{Introduction}

The Kerr-Anti-de Sitter (Kerr-AdS) black hole spacetimes $(\mathcal{M}, g)$ constitute a 2-parameter family of solutions to the celebrated Einstein equations

$$
\operatorname{Ric}_{\mu \nu}(g)-\frac{1}{2} \operatorname{R} g_{\mu \nu}+\Lambda g_{\mu \nu}=8 \pi T_{\mu \nu}
$$

in vacuum $\left(T_{\mu \nu}=0\right)$ and with negative cosmological constant $\Lambda<0$. The family (see already (2.14) for the metric) is parameterized by the black hole mass $M>0$, and specific angular momentum $a \neq 0$. The Kerr-AdS black holes posses a smooth Cauchy horizon beyond which the spacetime has infinitely many smooth extensions - thus violating determinism. Regular Cauchy horizons are thought, however, to be generically unstable, which is the content of the Strong Cosmic Censorship conjecture due to Roger Penrose [99]. Its strongest formulation, the $C^{0}$-formulation [14] (see already Conjecture 1), states that for generic initial data for (1.1), the metric cannot be continuously extended beyond a Cauchy horizon, in this sense saving determinism within classical general relativity. Unfortunately, for $\Lambda=0$ and $\Lambda>0$, this formulation was disproved by Dafermos-Luk [24]. However, a weaker formulation put forward by Christodoulou is still expected to be true (see already Conjecture 2). Refer to Sect. 1.1 for a more detailed discussion. For $\Lambda<0$, the question of the validity of the $C^{0}$-formulation of Strong Cosmic Censorship has until today remained open.

Motivated by the above, we study linear scalar perturbations $\psi$ of subextremal Kerr-AdS black holes solving the conformal scalar wave equation

$$
\square_{g} \psi-\frac{2}{3} \Lambda \psi=0
$$

which arise from smooth and compactly supported initial data posed on a spacelike hypersurface and which satisfy reflecting boundary conditions at infinity. We further assume that the black hole parameters satisfy the HawkingReall bound [52], see already (2.8). One can view (1.2) as a linear scalar analog of (1.1), and so the linear scalar analog of the $C^{0}$-formulation of Strong Cosmic Censorship is the statement that for generic black hole parameters, linear scalar perturbations $\psi$, arising from generic initial data for (1.2), fail to be continuous at the Cauchy horizon (see already Conjecture 3). 
Our main result Theorem 1 shows that if the dimensionless Kerr-AdS parameters mass $\mathfrak{m}=M \sqrt{-\Lambda}$ and angular momentum $\mathfrak{a}=a \sqrt{-\Lambda}$ satisfy a certain non-Diophantine condition, then linear scalar perturbations $\psi$ solving (1.2) and arising from generic initial data blow up

$$
|\psi| \rightarrow+\infty
$$

at the Cauchy horizon. We show that the set of such parameters is Baire-generic (but Lebesgue-exceptional).

Hence, our main result provides an - unexpected - positive resolution of the linear scalar analog of the $C^{0}$-formulation of the Strong Cosmic Censorship conjecture for $\Lambda<0$, provided that the genericity of the set of parameters is taken in the Baire-generic sense.

Theorem 1 is in sharp contrast to the result on Reissner-Nordström-AdS black holes [70] and to previous work on Strong Cosmic Censorship for $\Lambda \geq 0$-in both cases such perturbations $\psi$ were shown to remain bounded and to extend continuously across the Cauchy horizon.

The instability result (1.3) of Theorem 1 is not associated to superradiance (since the parameters satisfy the Hawking-Reall bound) and, more surprisingly, is also not a consequence of the well-known blue-shift instability [98] at the Cauchy horizon. Instead, Theorem 1 is a manifestation of the occurrence of small divisors originating from a new resonance phenomenon between, on the one hand, high frequencies associated to stable trapping on the exterior $[63,65]$ and, on the other hand, the poles of the interior scattering operator which are characteristic frequencies with respect to the Killing generator of the Cauchy horizon [72]. For this, it is fundamental that Kerr-AdS is rotating, as it is only in this case that stably trapped high frequency waves can, at the same time, be characteristic frequency waves with respect to the Killing generator of the Cauchy horizon. If now $\mathfrak{m}, \mathfrak{a}$ satisfy the non-Diophantine condition, then the resonance will be sufficiently strong (and the occurring divisors will be sufficiently small) so as to cause the instability (1.3).

Thus, in the case $\Lambda<0$, surprisingly, Diophantine approximation may turn out to be the elusive "Cosmic Censor" which Penrose was searching for in order to protect determinism in general relativity [99].

The story, however, has yet another level of complexity. We also conjecture that, if the dimensionless black hole parameters $\mathfrak{m}=M \sqrt{-\Lambda}$ and $\mathfrak{a}=a \sqrt{-\Lambda}$ satisfy a Diophantine condition, then linear scalar perturbations $\psi$ remain bounded $|\psi| \leq C$ at the Cauchy horizon. This would then hold for Lebesgue-generic but Baire-exceptional black hole parameters. If true, this would provide a negative resolution of the linear scalar analog of the $C^{0}$-formulation of Strong Cosmic Censorship provided that genericity of the parameters is now taken in the Lebesgue-generic sense. 
Returning to the fully nonlinear $C^{0}$-formulation of Strong Cosmic Censorship, the black hole parameters are themselves dynamic in evolution under (1.1). Thus, the above competing notions of genericity for the parameters may now be reflected in different formulations of the genericity assumption imposed on initial data in the statement of the conjecture. This could mean that the validity of Strong Cosmic Censorship is not only sensitive to the regularity of the extension but may also become highly sensitive to the precise notion of genericity imposed on the initial data.

\section{Outline of the introduction}

We begin in Sect. 1.1 with a presentation of the $C^{0}$-formulation (Conjecture 1) and Christodoulou's reformulation (Conjecture 2) of the Strong Cosmic Censorship conjecture. We also introduce their respective linear scalar analogs Conjecture 3 and Conjecture 4 and review the relevant previous work and difficulties for $\Lambda \geq 0$ and $\Lambda<0$. Then, turning to the Kerr-AdS case $(\Lambda<0)$, we will first outline in Sect. 1.2 the behavior of linear scalar perturbations on the black hole exterior before we focus on the interior in Sect. 1.3, see Fig. 1. In Sect. 1.4 we put both insights together and we will see, at least on a heuristic level, how small divisors and Diophantine approximation arise. This will lead to a new expectation that transcends Conjecture 3 and Conjecture 4 and which we formulate in Sect. 1.5 as Conjecture 5 and Conjecture 6. In Sect. 1.6 we state our main result Theorem 1, which resolves Conjecture 5 in the affirmative. Then, in Sect. 1.7 we give an outlook on Conjecture 6. In Sect. 1.8 we describe how we turn our heuristics of Sect. 1.4 into a proof of Theorem 1. Finally, we give a brief outline of the paper in Sect. 1.9.

\subsection{Strong Cosmic Censorship: Conjectures 1-4}

Recall from our previous discussion that our main motivation for studying linear perturbations on black hole interiors is to shed light on one of the most fundamental problems in general relativity: the existence of smooth Cauchy horizons.

In general, a Cauchy horizon $\mathcal{C H}$ defines the boundary beyond which initial data on a spacelike hypersurface (together with boundary conditions at infinity in the asymptotically AdS case) no longer uniquely determine the spacetime as a solution of the Einstein equations (1.1). The Kerr(-de Sitter or -Antide Sitter) black holes share the property that they indeed posses a smooth Cauchy horizon $\mathrm{CH}$ in their interiors. In particular, these spacetimes admit infinitely many smooth extensions beyond their Cauchy horizons solving (1.1), and in this sense violating determinism and the predictability of the theory. 
Fig. 1 (I): Exterior propagation, (II): Interior propagation

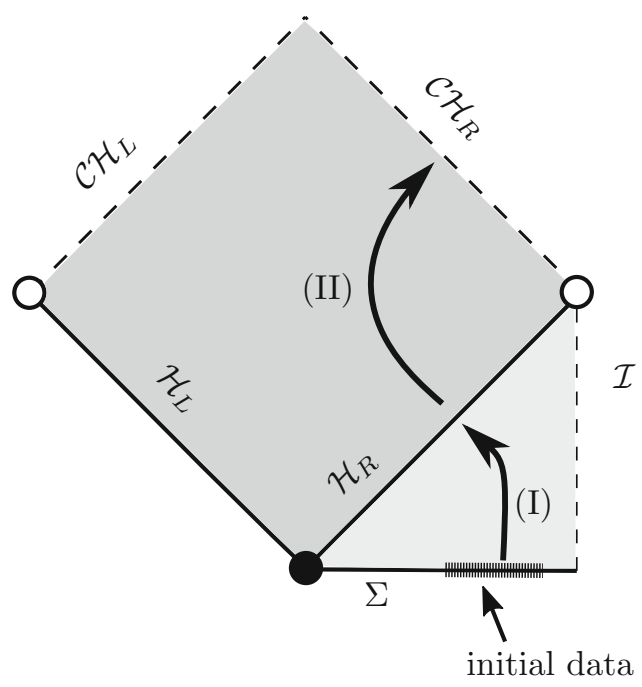

From a PDE point of view, this corresponds to a lack of global uniqueness for (1.1). However, the existence of regular Cauchy horizons is conjectured to be an artifact of the high degree of symmetry in those explicit spacetimes and generically it is expected that some sort of singularity ought to form at or before a Cauchy horizon. The original mechanism which was invoked to support this expectation is a blue-shift instability associated to Cauchy horizons [98]. The emergence of such a singularity at or before a Cauchy horizon is paradoxically "good" because-if sufficiently strong-it can be argued that this restores determinism, as the fate of any observer approaching the singularity, though bleak, is uniquely determined. Making this precise gives rise to various formulations of what is known as the Strong Cosmic Censorship (SCC) conjecture [16,99].

We begin with the $C^{0}$-formulation of the SCC conjecture which can be seen as the strongest, most desirable, inextendibility statement in this context.

Conjecture 1 ( $C^{0}$-formulation of Strong Cosmic Censorship). For generic compact, asymptotically flat or asymptotically Anti-de Sitter vacuum initial data, the maximal Cauchy development of (1.1) is inextendible as a Lorentzian manifold with $C^{0}$ metric.

This formulation is related to the statement that observers are torn apart by infinite tidal deformations before they have the chance to cross a Cauchy horizon [24,95].

Surprisingly, the $C^{0}$-formulation (Conjecture 1) was recently proved to be false for both cases $\Lambda=0$ and $\Lambda>0$ [24] (see discussion later). The reason is that it turns out that the blue-shift instability is not sufficiently strong to destroy the metric itself, only derivatives of the metric. However, the following weaker, 
yet still well-motivated, formulation introduced by Christodoulou in [16] is still expected to hold true (though for the $\Lambda>0$ case see the discussion later).

Conjecture 2 (Christodoulou's reformulation of Strong Cosmic Censorship). For generic compact, asymptotically flat or asymptotically Anti-de Sitter vacuum initial data, the maximal Cauchy development of (1.1) is inextendible as a Lorentzian manifold with $C^{0}$ metric and locally square integrable Christoffel symbols.

Unlike the $C^{0}$-formulation in Conjecture 1, the statement of Conjecture 2 does not guarantee the complete destruction of observers approaching Cauchy horizons. However, it restores determinism in the sense that even just weak solutions must break down at Cauchy horizons. Nonetheless, one may remain uneasy as to whether the standard notion of weak solution to (1.1) is finally the correct one $[78,82,97]$. In this sense it is a pity that Conjecture 1 turned out to be false in the $\Lambda \geq 0$ cases, as it would have provided a much more definitive resolution of the spirit of the Strong Cosmic Censorship conjecture. Hence, it is of interest to know whether the situation is better in the $\Lambda<0$ case!

\section{Linear scalar analog of the Strong Cosmic Censorship conjecture}

The aforementioned formulations of SCC have linear scalar analogs on the level of (1.2). Indeed, under the identification $\psi \sim g$, the linear scalar wave equation (1.2) can be seen as a naive linearization of the Einstein equations (1.1) after neglecting the nonlinearities and the tensorial structure. Moreover, many phenomena and difficulties for the full Einstein equations (1.1) are already present at the level of (1.2).

The linear scalar analog of Conjecture 1 in a neighborhood of Kerr and Kerr-(Anti-)de Sitter corresponds to the statement that for generic black hole parameters, linear scalar perturbations $\psi$ arising from generic data on a spacelike hypersurface solving (1.2) blow up in amplitude at the Cauchy horizon.

Conjecture 3 (Linear scalar analog of the $C^{0}$-formulation of SCC (Conjecture 1)). For generic Kerr-(dS/AdS) black hole parameters, linear scalar perturbations $\bar{\psi}$ solving (1.2), arising from generic initial data, blow up in amplitude

$$
|\psi| \rightarrow+\infty
$$

at the Cauchy horizon.

The reformulation due to Christodoulou (Conjecture 2) finds its linear scalar analog in the $H_{\text {loc }}^{1}$ blow up of $\psi$ at the Cauchy horizon in view of the identification $\partial \psi \sim \Gamma$. 
Conjecture 4 (Linear scalar analog of Christodoulou's reformulation of SCC (Conjecture 2)). For generic Kerr-(dS/AdS) black hole parameters, linear

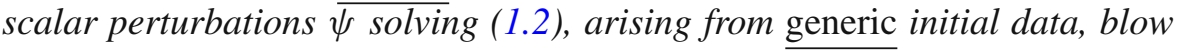
up in local energy

$$
\|\psi\|_{H_{\mathrm{loc}}^{1}}=+\infty
$$

at the Cauchy horizon.

The word generic appears twice in the above formulations, both in the context of the parameters and in the context of the perturbation. This is because in the fully nonlinear Conjecture 1 and Conjecture 2, the background parameters are themselves dynamic in evolution under (1.1) and thus both would be encompassed in the genericity of the initial data.

Genericity of the black hole parameters. As we will show in the present paper, for the Kerr-AdS case, the validity of Conjecture 3 and Conjecture 4 will depend in a crucial way on the notion of genericity (Baire-generic or Lebesgue-generic) imposed on the parameters. This will eventually lead us to refine the above statements of Conjecture 3 and Conjecture 4 (see already Sect. 1.5).

Genericity of the initial data. We will assume that the initial data lie in the class of smooth functions of compact support. Regarding genericity within that class, note that just finding one single solution for which the blow-up statement is true already yields a natural notion of genericity. Indeed, since (1.2) is linear, it would then follow that data for which the arising solution does not blow up satisfy a co-dimension 1 property (see already Remark 1.1) and thus, would be exceptional. It is this notion of genericity of the initial data which we will consider later in Sect. 1.5. Note that we will also consider a more refined notion of genericity of initial data in Remark 1.5.

In the above discussion, one may also consider the Reissner-Nordström(dS/AdS) spacetimes (see e.g. [69]) which are spherically symmetric electrovacuum solutions to (1.1). Reissner-Nordström(-dS/AdS) spacetimes are often studied as a toy model for Kerr(-dS/AdS) and the above Conjecture 3 and Conjecture 4 can also be formulated replacing Kerr(-dS/AdS) with ReissnerNordström(-dS/AdS).

Before we bring our discussion of SCC to asymptotically AdS black holes $(\Lambda<0)$, we will first review the state of the art of the SCC conjecture for the cases $\Lambda=0$ and $\Lambda>0$.

$\operatorname{SCC}$ for $\Lambda=0$ and $\Lambda>0$

Linear level. The definitive negative resolution of the fully nonlinear Conjecture 1 in [24] for both $\Lambda=0$ and $\Lambda>0$ was preceded by the negative 
resolution of the linear Conjecture 3 in $[41,42,54]$ for $\Lambda=0$ and in $[18,56]$ for $\Lambda>0$. It was shown that solutions of (1.2) arising from regular and localized data on a spacelike hypersurface remain continuous and uniformly bounded $|\psi| \leq C$ at the Cauchy horizon for all subextremal Kerr black hole interiors $(\Lambda=0)$, and very slowly rotating subextremal Kerr-dS black hole interiorshence disproving Conjecture 3 for $\Lambda=0$ and $\Lambda>0$. (For the extremal case see $[45,46]$ and for the Schwarzschild case see [39].) The key ingredient in showing boundedness at the Cauchy horizon is a sufficiently fast decay (polynomial with rate $v^{-p}$ with $p>1$ for $\Lambda=0$ and exponential for $\Lambda>0$ ) of linear scalar perturbations along the event horizon. Using suitable energy estimates associated to the red-shift vector field introduced in [25] and the vector field $S=|u|^{p} \partial_{u}+|v|^{p} \partial_{v}$, this decay is then propagated into the black hole all the way up to the Cauchy horizon $\mathcal{C H}$, where it is sufficient to conclude uniform boundedness. We remark already that this method manifestly fails for asymptotically AdS black holes, where linear scalar perturbations decay merely at a logarithmic rate along the event horizon $[63,65]$.

While Conjecture 3 is false for $\Lambda=0$, as remarked above, at least the weaker formulation Conjecture 4 holds true: It was proved that the (non-degenerate) local energy at the Cauchy horizon blows up, $\|\psi\|_{H_{\text {loc }}^{1}}=+\infty$, for a generic set of solutions $\psi$ on Reissner-Nordström [79] and Kerr [26] black holes in the full subextremal range of parameters. A similar blow-up behavior was obtained for Kerr in [83] assuming lower bounds (which were shown later in [55] to indeed hold generically) on the energy decay rate of a solution along the event horizon. These results thus also support the validity of the fully nonlinear Conjecture 2 for $\Lambda=0$.

On the other hand, in the $\Lambda>0$ case, the exponential convergence of perturbations along the event horizon of a Kerr-de Sitter black hole is in direct competition with the exponential blue-shift instability near the Cauchy horizon. Thus, the question of the validity of Conjecture 4 becomes even more subtle for $\Lambda>0$ and has received lots of attention in the recent literature. We refer to the conjecture in [22], the survey article [103], the recent results $[18,27-30]$ and the works $[58,59]$ taking also quantum effects into account.

Another related result, which will turn out to be important for the paper at hand, is proved in work of the author and Shlapentokh-Rothman [72]: The main theorem establishes a finite energy scattering theory for solutions of (1.2) on the interior of Reissner-Nordström. In this scattering theory, a linear isomorphism between the degenerate energy spaces (associated to the Killing field $T$ ) corresponding to the event and Cauchy horizon is established. The problem reduces to showing uniform bounds for the transmission and reflection coefficients $\mathfrak{T}(\omega, \ell)$ and $\mathfrak{R}(\omega, \ell)$ for fixed frequency solutions. Formally, for an incoming wave at the right event horizon $\mathcal{H}_{R}$, the transmission and reflection coefficients correspond to the amount of $T$-energy scattered to the left and right 
Cauchy horizon $\mathcal{C H}_{L}$ and $\mathcal{C H}_{R}$, respectively. Indeed, the theory also carries over to non-zero cosmological constant $\Lambda \neq 0$ except for the characteristic frequency $(\omega=0)$ associated to $T$, thought of now as the generator of the Cauchy horizon. (Note that these results are compatible with the blow-up of the local energy at the Cauchy horizon [79] because of the degeneracy of the $T$-energy.) These insights will turn out to be important for the interior analysis of the present paper, see already Sect. 1.3.

Nonlinear level. Turning to the nonlinear problem of (1.1), Dafermos-Luk proved the full nonlinear $C^{0}$-stability of the Kerr Cauchy horizon in [24]. Their work definitively disproves Conjecture 1 for $\Lambda=0$ (subject only to the completion of a proof of the nonlinear stability of the Kerr exterior). Mutatis mutandis, their proof of $C^{0}$-stability also applies to Kerr-de Sitter Cauchy horizons, where the exterior has been shown to be nonlinearly stable in the very slowly rotating case [57]. This unconditionally disproves Conjecture 1 for $\Lambda>0$.

Nonlinear inextendibility results at Cauchy horizons have been proved only in spherical symmetry: For the Einstein-Maxwell-scalar field system, the Cauchy horizon is shown to be $C^{2}$ unstable $[21,80,81]$ for a generic set of spherically symmetric initial data. See also the pioneering work in $[95,100]$ and the more general results on the Einstein-Maxwell-charged scalar field system in [71,112-114]. This proves the $C^{2}$-formulation of SCC, and by very recent work [108], the $C^{0,1}$-formulation (but not yet Conjecture 2) in spherical symmetry. For work in the $\Lambda>0$ case see $[19,20]$. The question of any type of nonlinear instability of the Cauchy horizon without symmetry assumptions and the validity of Conjecture 2 (even restricted to a neighborhood of Kerr) have yet to be understood.

We shall also mention that for $T^{3}$-Gowdy spacetimes the $C^{2}$-formulation of SCC has been shown in $[106,107]$. Further, in the context of Bianchi systems [34] (which can be formulated as finite dimensional dynamical systems [53, $115]$ ), a $C^{2}$-formulation of SCC has been shown for generic data in $[104,105]$ for Bianchi A and in [102] for Bianchi B systems. In the dynamical system formulation, Baire-genericity has been crucial to the argument, see e.g. [102, Sect. 1.4].

\section{SCC for asymptotically AdS spacetimes $\Lambda<0$}

As we shall see in the present paper, the situation for asymptotically AdS black holes with $\Lambda<0$ will turn out to be radically different.

First, in view of the lack of global hyperbolicity of asymptotically AdS spacetimes, one needs to specify additional boundary conditions at infinity (at $\mathcal{I})$ to guarantee well-posedness of $(1.1)$ and $(1.2)$, see $[40,44,49,61,118]$. The most natural in this context are reflecting (Dirichlet) boundary conditions [44]. 
Fig. 2 Penrose diagram of the maximal Cauchy development of Kerr-AdS data on a spacelike surface $\Sigma$ with Dirichlet (reflecting) boundary conditions prescribed on null infinity $\mathcal{I}$

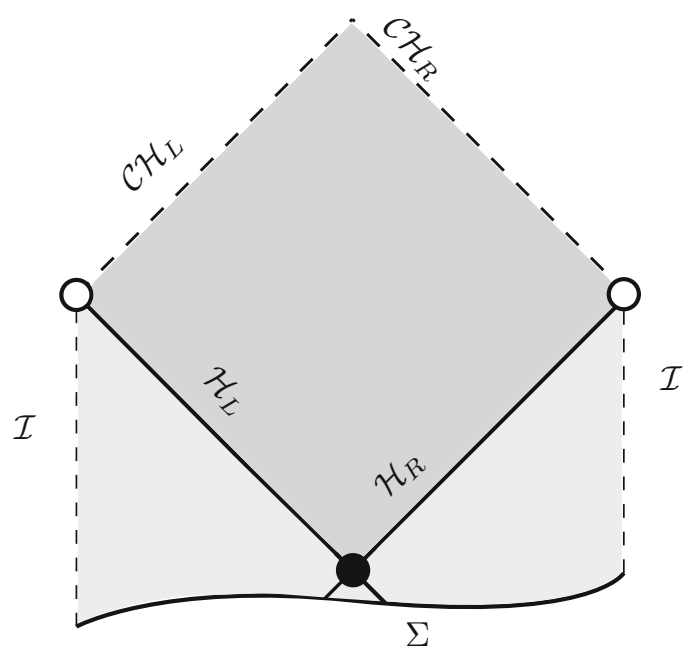

In what follows we will assume such Dirichlet boundary conditions. (Refer to Sects. 1.5 and 1.6 later for remarks on more general boundary conditions.)

We first discuss linear scalar perturbations solving (1.2) arising from data posed on a spacelike hypersurface on asymptotically AdS black holes. In contrast to $\Lambda \geq 0$, where linear scalar perturbations $\psi$ decay at a polynomial $(\Lambda=0)$ and exponential $(\Lambda>0)$ rate, linear scalar perturbations $\psi$ of KerrAdS (and Reissner-Nordström-AdS) decay merely at a logarithmic rate on the exterior as proved in $[63,65] .{ }^{1}$ The origin of this slow decay is a stable trapping phenomenon of high-frequency waves traveling along stably trapped null geodesics which repeatedly bounce off null infinity $\mathcal{I}$. (Contrast this with the work $[9,31,96]$ in $2+1$ dimensions and refer to [10] for a discussion of the Ori model for Reissner-Nordström-AdS.) For 5D asymptotically flat black holes, a similar log-decay result was shown in [4], which also relies on the existence of stably trapped null geodesics.

With the logarithmic decay on the exterior in hand, we first recall from the discussion above that in the $\Lambda \geq 0$ cases Conjecture 3 is false (and, in fact, so is the fully nonlinear Conjecture 1), yet at least in the $\Lambda=0$ case Conjecture 4 is true (and, hopefully, Conjecture 2 as well). Indeed, our methods in principle also show Conjecture 4 for $\Lambda<0$. However, in view of the slower decay in the case $\Lambda<0$, one might suspect a stronger instability at the Cauchy horizon in this case. This raises the attractive possibility that Conjecture 1 and Conjecture 3 might actually be true for $\Lambda<0$, which would give a more

\footnotetext{
1 Recall that we restrict our attention to Kerr black holes below the Hawking-Reall bound [52] as otherwise growing modes are shown to exist [33].
} 
definitive resolution to the issue of Strong Cosmic Censorship than the weaker Conjecture 2 and Conjecture 4.

For the Reissner-Nordström-AdS spacetime, which is often considered as a toy model of Kerr-AdS, this question was first taken up in [70]. For that case, however, the hopes expressed in the above paragraph were not fulfilled! It was shown in [70] that, despite the slow decay on the exterior, all linear scalar perturbations $\psi$ on Reissner-Nordström-AdS (in the full subextremal range) remain uniformly bounded, $|\psi| \leq C$, on the interior and extend continuously across the Cauchy horizon. Thus, the Reissner-Nordström analog of Conjecture 3 is false. To understand the additional phenomenon which was exploited to prove boundedness, let us decompose a linear scalar perturbation $\psi$ into frequencies $\omega, m, \ell$ associated to the separation of variables. On the exterior, it is the high frequency part (i.e. $|\omega|,|m|, \ell$ large) of $\psi$ which is exposed to stable trapping and decays slowly, whereas the low frequency part $(|\omega|,|m|, \ell$ small) decays superpolynomially. In the interior, however, the main obstruction to boundedness is the interior scattering pole which is located at the characteristic frequency $\omega=0$ with respect to $T$, now thought of as the Killing generator of the Cauchy horizon. (Refer also to the discussion in [72, Sect. 3.6].) Thus, for Reissner-Nordström-AdS, the slowly decaying part of $\psi$ is decoupled in frequency space from the part susceptible to the interior scattering pole at $\omega=0$. (See already Fig. 6.) The above result on ReissnerNordström-AdS may suggest that, just as in the cases of $\Lambda \geq 0$, Conjecture 3 is false for $\Lambda<0$, albeit for different reasons.

The present paper on Kerr-AdS, however, provides an unexpected positive resolution of Conjecture 3 for $\Lambda<0$. We show in Theorem 1 that there

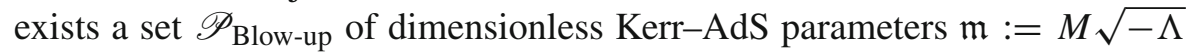
and $\mathfrak{a}:=a \sqrt{-\Lambda}$ which is Baire-generic but Lebesgue-exceptional, such that on all Kerr-AdS black hole whose parameters lie in $\mathscr{P}_{\text {Blow-up, generic linear }}$ scalar perturbations $\psi$ blow up $|\psi| \rightarrow+\infty$ at the Cauchy horizon. Thus, our main result Theorem 1 shows that Conjecture 3 is true if Baire-genericity is imposed on the Kerr-AdS parameters.

This set of parameters is defined through a non-Diophantine condition. This condition arises from small divisors originating from a resonance phenomenon between, on the one hand, specific high frequencies associated to stable trapping on the exterior and, on the other hand, poles of the interior scattering operator which are characteristic frequencies with respect to the Killing generator of the Cauchy horizon. This resonance phenomenon is possible because the characteristic frequencies of the Cauchy horizon are now the frequencies

$$
\omega-\omega \_m=0,
$$


where

$$
\omega_{-}=\frac{a\left(1+a^{2} \Lambda / 3\right)}{r_{-}^{2} a^{2}}
$$

is the frequency at which the Cauchy horizon rotates. In contrast to ReissnerNordström, (1.6) can now be satisfied for frequencies $|\omega|,|m|, \ell$ which are large. It is not all high frequencies, however, but only specific high frequencies, so-called quasimode (on the real axis) or quasinormal mode (in the complex plane) frequencies $\left(\omega_{n}, m_{n}, \ell_{n}\right)_{n \in \mathbb{N}}$ which are responsible for the slow decay on the exterior. (See already Sect. 1.2.) This resonance phenomenon will lead to small divisors of the form $\frac{1}{\omega_{n}-\omega_{-} m_{n}}$. Now, if the specific quasinormal mode frequencies approximate the characteristic frequencies $\omega=\omega_{-} m$ sufficiently well, i.e. if $\left|\omega_{n}-\omega_{-} m_{n}\right|$ is sufficiently small for infinitely many $n \in \mathbb{N}$, then we will show that generic linear scalar perturbations $\psi$ of Kerr-AdS blow up $|\psi| \rightarrow \infty$ at the Cauchy horizon. This naturally leads to a non-Diophantine condition on the black hole parameters $\mathfrak{m}, \mathfrak{a}$ which, as we will show, holds true for a set of parameters $\mathfrak{m}, \mathfrak{a}$ which is Baire-generic but Lebesgue-exceptional.

The above result is not the last word on Conjecture 3 on Kerr-AdS black holes. We also complement our main result with the conjecture that if the parameters $\mathfrak{m}, \mathfrak{a}$ satisfy a complementary Diophantine condition, then the resonance phenomenon outlined above is "weak" and linear scalar perturbations $\psi$ remain bounded $|\psi| \leq C$ at the Cauchy horizon. This would then hold for black hole parameters which lie in a set $\mathscr{P}_{\text {Bounded }}$ which is Baire-exceptional but Lebesgue-generic. Thus, we expect Conjecture 3 to be false if Lebesguegenericity is imposed on the Kerr-AdS parameters.

Since the parameters are dynamic in the full nonlinear (1.1), this suggests that for $\Lambda<0$ the validity of the $C^{0}$-formulation of Strong Cosmic Censorship (Conjecture 1) may change in a spectacular way according to the notion of genericity imposed.

Instability of asymptotically AdS spacetimes? If we accept to interpret the above results as supporting Conjecture 1, they leave determinism in better shape for $\Lambda<0$ compared to the $\Lambda \geq 0$ cases. However, turning to the fully nonlinear dynamics governed by (1.1), there is yet another scenario which could happen. While Minkowski space $(\Lambda=0)$ and de Sitter space $(\Lambda>0)$ have been proved to be nonlinearly stable [17,43], Anti-de Sitter space $(\Lambda<$ 0 ) is expected to be nonlinearly unstable with Dirichlet conditions imposed at infinity. This was recently proved by Moschidis [84-87] for appropriate matter models. See also the original conjecture in [23] and the numerical results in [11]. Similarly, for Kerr-AdS (or Reissner-Nordström-AdS), the slow logarithmic decay on the linear level proved in [65] could in fact give rise to nonlinear instabilities in the exterior. (Note that in contrast, nonlinear 
stability for spherically symmetric perturbations of Schwarzschild-AdS was shown for Einstein-Klein-Gordon systems [64].) If indeed the exterior of Kerr-AdS was nonlinearly unstable, the linear analysis on the level of (1.2) could not serve as a model for (1.1) and the question of the validity of Strong Cosmic Censorship would be thrown even more open!

\subsection{Exterior: log-decay, quasi(normal) modes and semi-classical analysis}

We recall the result of Holzegel-Smulevici $[63,65]$ that linear scalar perturbations $\psi$ solving (1.2) decay at a sharp inverse logarithmic rate

$$
|\psi| \leq \frac{C}{\log (t)}
$$

on the Kerr-AdS exterior. (For smooth initial data, the decay in (1.8) can be slightly improved to $|\psi| \leq \frac{C_{n}}{\log ^{n}(t)}$ for $n \in \mathbb{N}$.) The reason for the slow decay is the stable trapping phenomenon near infinity discussed earlier. One manifestation of this phenomenon is the existence of so-called quasimodes and quasinormal modes which are "converging exponentially fast" to the real axis. Note already that in the proof of Theorem 1 we will work with quasimode frequencies but we will not make use of a quasinormal mode construction or decomposition. However, quasinormal modes provide perhaps the simplest route to obtain some intuition-paired with the interior analysis in Sect. 1.3for how the relation to Diophantine approximation arises. Our discussion of quasi(normal) modes starts with the property that (1.2) is formally separable [13].

Separation of Variables. With the fixed-frequency ansatz

$$
\psi=\frac{u(r)}{\sqrt{r^{2}+a^{2}}} S_{m \ell}(a \omega, \cos \theta) e^{i m \phi} e^{-i \omega t},
$$

the wave equation (1.2) reduces to a coupled system of o.d.e's (see already (2.43)). The radial o.d.e. reads

$$
-u^{\prime \prime}\left(r^{*}\right)+\tilde{V}\left(r^{*}, \omega, \lambda_{m \ell}\right) u=0
$$

for a rescaled radial variable $r^{*} \in\left(-\infty, \frac{\pi}{2} l\right)$ with $r^{*}\left(r=r_{+}\right)=-\infty, r^{*}(r=$ $+\infty)=\frac{\pi}{2} l$. The radial o.d.e (1.10) couples to the angular o.d.e. through the potential $\tilde{V}$ which depends on the eigenvalues $\lambda_{m \ell}(a \omega)$ of the angular o.d.e.

$$
P(a \omega) S_{m \ell}(a \omega, \cos \theta)=\lambda_{m \ell}(a \omega) S_{m \ell}(a \omega, \cos \theta),
$$


where $P(a \omega)$ is a self-adjoint Sturm-Liouville operator. The radial o.d.e. (1.10) is equipped with suitable boundary conditions at $r^{*}=-\infty$ and $r^{*}=\frac{\pi}{2} l$ which stem from imposing regularity for $\psi$ at the event horizon and Dirichlet boundary conditions at infinity. This leads to the concept of a mode solution $\psi$ of (1.2) defined to be of the form (1.9) such that $u$ solves (1.10) and $S_{m \ell}$ solves (1.11) with the appropriate boundary conditions imposed. If such a solution $\psi$ were to exist for $\omega \in \mathbb{R}$, this would correspond to a time-periodic solution. Such solutions are however incompatible with the fact that all admissible solutions decay. Nevertheless, there exist "almost solutions" which are time-periodic. This leads us to the concept of

Quasimodes. In [65] it was shown that there exists a set of real frequencies $\left(\omega_{n}, m_{n}=0, \ell_{n}\right)_{n \in \mathbb{N}}$ such that the corresponding functions $\psi_{n}$ "almost" solve (1.2) in the sense that they satisfy $\square_{g} \psi_{n}+\frac{2}{3} \Lambda \psi_{n}=F_{n}$ with $\left|F_{n}\right| \lesssim \exp \left(-c \ell_{n}\right)$. These almost-solutions are called quasimodes and their existence actually implies that the logarithmic decay of [63] is sharp as shown in [65]. These quasimode frequencies are equivalently characterized by the condition that the Wronskian $\mathfrak{W}\left[u_{\mathcal{H}^{+}}, u_{\infty}\right]$ of solutions $u_{\mathcal{H}^{+}}, u_{\infty}$ of (1.10) adapted to the boundary conditions satisfies

$$
\left|\mathfrak{W}\left[u_{\mathcal{H}^{+}}, u_{\infty}\right]\left(\omega_{n}, m_{n}, \ell_{n}\right)\right| \lesssim e^{-c \ell_{n}}
$$

The reason why there exist such quasimodes is that in the high frequency limit, the potential in (1.10) admits a region of stable trapping, see already Fig. 3. Alternatively and intimately related to the above, the existence of quasimodes can be seen as a consequence of the existence of stably trapped null geodesics on the exterior of asymptotically AdS black holes.

Quasinormal modes. The Wronskian $\mathfrak{W}\left[u_{\mathcal{H}^{+}}, u_{\infty}\right]$ has no real zeros, $\mathfrak{W}\left[u_{\mathcal{H}^{+}}, u_{\infty}\right] \neq 0$, however, it might very well have zeros in the lower halfplane with $\operatorname{Im}(\omega)<0$. These zeros correspond to so-called quasinormal modes i.e. solutions of the form (1.9) which decay in time at an exponential rate. Note that quasinormal modes do not have finite energy on $\{t=$ const. $\}$ slices (in particular they have infinite energy on $\Sigma_{0}=\{t=0\}$ ). However, they have finite energy for $\left\{t^{*}=\right.$ const. $\}$-slices, where $t^{*}$ is a suitable time coordinate which extends regularly to the event horizon $\mathcal{H}_{R}$, see already (2.25). For a more precise definition, construction and a more detailed discussion of quasinormal modes in general we refer to [47]. Turning back to Kerr-AdS, we note that the bound (1.12) implies the existence of zeros of $\mathfrak{W}\left[u_{\mathcal{H}^{+}}, u_{\infty}\right]$ exponentially close to the real axis as shown in [48], see also [119]. More precisely, it was shown that there exist axisymmetric quasinormal modes with frequencies $m=0$ and $(\omega, \ell)=\left(\omega_{n}, \ell_{n}\right)_{n \in \mathbb{N}}$ satisfying

$$
c \ell_{n} \leq\left|\operatorname{Re}\left(\omega_{n}\right)\right| \leq C \ell_{n},
$$




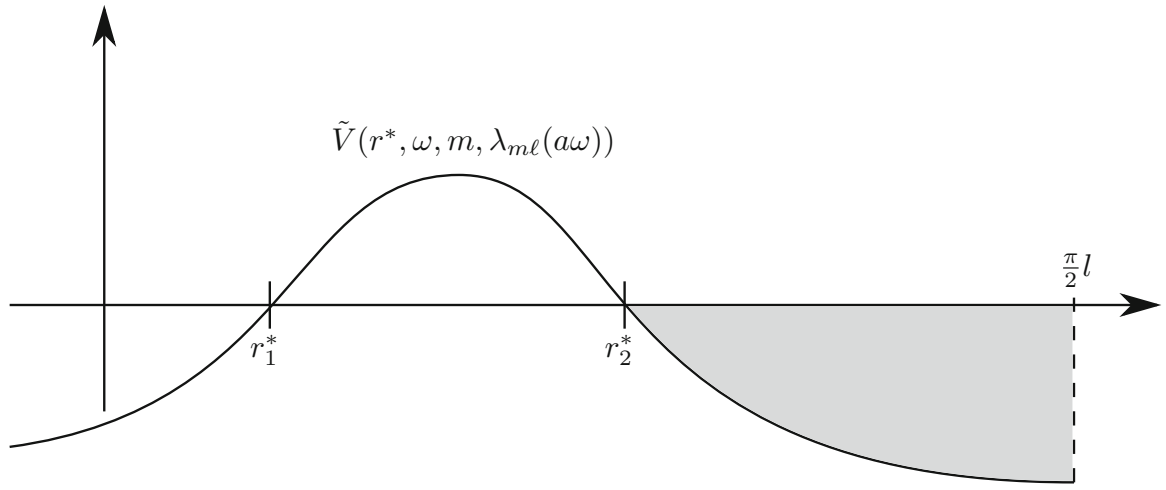

Fig. 3 Potential $\tilde{V}$ with frequency $\omega, m, \ell$ for which we expect quasimodes. The gray area is a suitable projection of the phase space volume

$$
0<-\operatorname{Im}\left(\omega_{n}\right) \leq C \exp \left(-c \ell_{n}\right)
$$

While the previous results were proved in axisymmetry to simplify the analysis, in principle, they also extend to non-axisymmetric solutions as remarked in [48].

Semi-classical heuristics for distribution of quasimodes and quasinormal modes. We first turn to the heuristic distribution of the quasimode frequencies in the semi-classical (high frequency) limit. For large $|m|, m \in \mathbb{Z}, \ell \geq|m|$, we expect a quasimode with frequencies $m, \ell, \omega$ to exist, if the potential $\tilde{V}\left(r^{*}, \omega, m, \lambda_{m \ell}(a \omega)\right)$ appearing in the radial o.d.e. (1.10) satisfies (see Fig. 3)

- $\tilde{V}\left(r^{*}, \omega, m, \lambda_{m \ell}(a \omega)\right)>0$ for $r_{1}^{*}<r^{*}<r_{2}^{*}$,

- $\tilde{V}\left(r^{*}, \omega, m, \lambda_{m \ell}(a \omega)<0\right.$ for $r_{2}^{*}<r^{*} \leq \frac{\pi}{2} l$.

Note that the conditions above are satisfied for a range of $\omega$ of the form $c \ell<|\omega|<C \ell$. In addition, for a quasimode to exist, the potential has to satisfy the Bohr-Sommerfeld quantization condition (see e.g. [76, Chapter VII]). In our case this means that the phase space volume

$$
\frac{1}{2 \pi} \operatorname{vol}\left\{\left(r^{*}, \xi\right): \xi^{2}+\tilde{V}\left(r^{*}, \omega, m, \lambda_{m \ell}(a \omega)\right)<0, r^{*}>r_{2}^{*}\right\}
$$

should be an integer multiple modulo the Maslov index up to an exponentially small error.

Thus, at least heuristically, we expect that for given but large $|m|, \ell \geq$ $|m|$, there exist $N(m, \ell) \sim \ell$ intervals of quasimodes with midpoint $\omega \sim \ell$ and length $e^{-c \ell}$. While quasimode frequencies are defined through an open condition (c.f. (1.12)), quasinormal mode frequencies will be discrete and in an exponentially small neighborhood of quasimodes. Thus, we expect the 


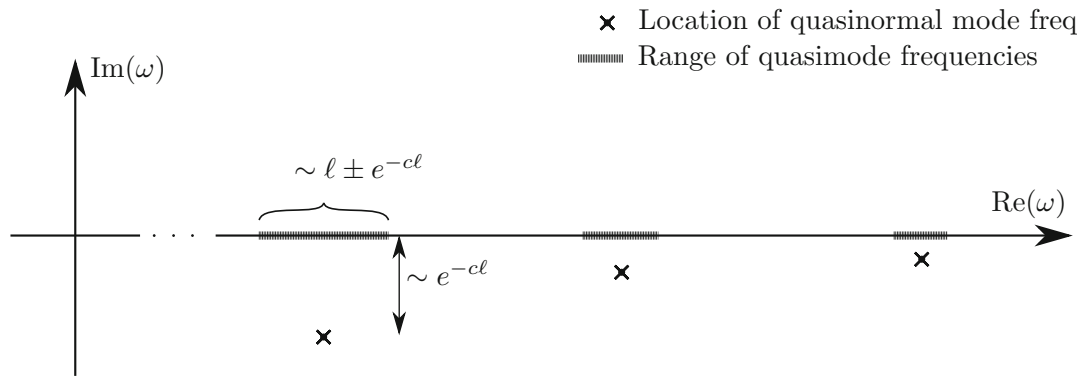

Fig. 4 Quasimodes and quasinormal modes frequencies for large $|m| \sim \ell$

quasinormal mode frequencies to be distributed as

$$
\begin{aligned}
& c \ell \leq\left|\operatorname{Re}\left(\omega_{m \ell n}\right)\right| \leq C \ell, \\
& 0<-\operatorname{Im}\left(\omega_{m \ell n}\right) \leq C \exp (-c \ell) .
\end{aligned}
$$

Refer to Fig. 4 for a visualization of the expected distribution of quasimodes and quasinormal modes.

For our heuristic analysis we will now consider a solution $\psi$ of (1.2) which consists of an infinite sum of weighted quasinormal modes (Warning: A general solution cannot be written as a sum of quasinormal modes.)

$$
\begin{aligned}
& \psi(r, t, \theta, \phi)=\sum_{m \in \mathbb{Z}} \sum_{\ell \geq|m|} \sum_{n=1}^{N(m, \ell)} \tilde{a}(m, \ell, n) \frac{u\left(r, \omega_{m \ell n}, m, \ell\right)}{\sqrt{r^{2}+a^{2}}} e^{-i \omega_{m \ell n} t} \\
& S_{m \ell}\left(a \omega_{m \ell n}, \cos \theta\right) e^{i m \phi},
\end{aligned}
$$

where we require that the weights $\tilde{a}(m, \ell, n)$ have superpolynomial decay. This ensures that the initial data (posed on a $\left\{t^{*}=\right.$ const. $\}$-slice) are smooth where we assume that each individual quasinormal mode is suitably normalized. ${ }^{2}$ Restricting this solution $\psi$ to the event horizon yields

$$
\begin{gathered}
\psi\left\lceil_{\mathcal{H}}\left(v, \theta, \tilde{\phi}_{+}\right)=\sum_{m \in \mathbb{Z}} \sum_{\ell \geq|m|} \sum_{n=1}^{N(m, \ell)} a(m, \ell, n) e^{-i \omega_{m \ell n} v}\right. \\
S_{m \ell}\left(a \omega_{m \ell n}, \cos \theta\right) e^{i m \tilde{\phi}_{+}}
\end{gathered}
$$

for new coefficients $a(m, \ell, n)$ which satisfy $|a(m, \ell, n)| \sim \mid \tilde{a}(m, \ell, n)$ $u\left(r_{+}, \omega_{m \ell n}, m, \ell\right) \mid$. Now, note that the radial part of the quasinormal mode

\footnotetext{
2 By a domain of dependence argument one can then produce a solution arising from smooth data on $\Sigma_{0}$.
} 
Fig. 5 Interior scattering $\mathfrak{S}_{\mathcal{H}_{R} \rightarrow \mathcal{C H}_{R}}$ from event horizon $\mathcal{H}_{R}$ to Cauchy horizon $\mathrm{CH}_{R}$

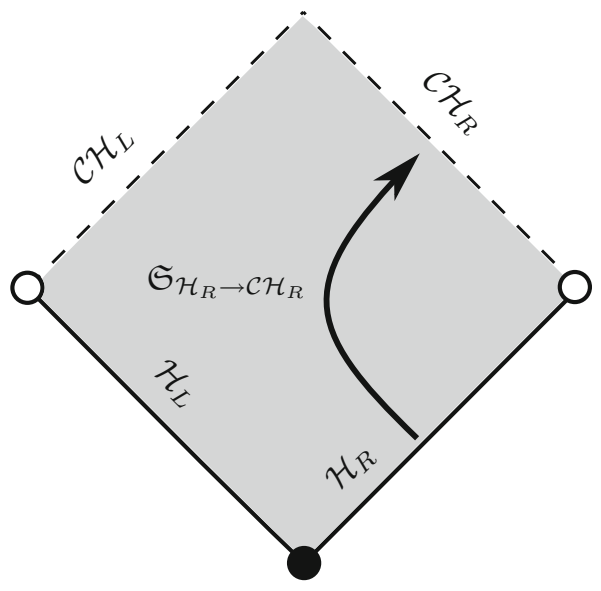

$\left|u\left(r, \omega_{m \ell n}, m, \ell\right)\right|$ will be localized in the region of stable trapping, i.e. in the region $\left\{r^{*} \geq r_{2}^{*}\right\}$ of Fig. 3. From semi-classical heuristics, we expect that only an exponentially damped proportion "tunnels" from the region of stable trapping through the barrier to event horizon at $r=r_{+}$. More precisely, the damping factor of the exponent of $\left|u\left(r_{+}, \omega_{m \ell n}, m, \ell\right)\right|$ is expected to be proportional to

$$
\int_{r_{1}^{*}}^{r_{2}^{*}} \sqrt{\tilde{V}\left(r^{*}, \omega_{R}, m, \lambda_{m \ell}\left(a \omega_{R}\right)\right)} \mathrm{d} r^{*} \sim \ell .
$$

Now, for any choice of superpolynomially decaying (or polynomially decaying) weights $\tilde{a}(m, \ell, n)$, the new coefficients $a(m, \ell, n)$ decay exponentially

$$
|a(m, \ell, n)| \lesssim \exp (-C \ell) .
$$

Thus, choosing coefficients $\tilde{a}(m, \ell, n)$ now corresponds to choosing coefficients $a(m, \ell, n)$ satisfying (1.20) and vice versa. In view of this, instead of choosing $\tilde{a}(m, \ell, n)$, we will go forward in our heuristic discussion by choosing coefficients $a(m, \ell, n)$ satisfying (1.20). The goal is to choose such coefficients such that $\psi$ blows up at the Cauchy horizon!

\subsection{Interior: scattering from event to Cauchy horizon}

We now turn to the interior problem. We will view some aspects of the propagation of $\psi$ from the event horizon to the Cauchy horizon as a scattering problem as visualized in Fig. 5. We refer to [72] for a detailed discussion of the scattering problem on black hole interiors. Unlike in [72], we will not develop a full scattering theory for Kerr-AdS, but rather make use of a key insight from 
[72] adapted to our context. Recall from [72, Proposition 6.2] that on ReissnerNordström-AdS, the scattering operator $\mathfrak{S}_{\mathcal{H}_{R} \rightarrow \mathcal{C H}_{R}}$ in the interior has a pole at the frequency $\omega=0$, which is the characteristic frequency associated to the Killing generator of the Cauchy horizon $T$. In the present case for Kerr-AdS, it is the vector field $K_{-}:=T+\omega_{-} \Phi$ which generates the Cauchy horizon and thus the characteristic frequencies are those satisfying $\omega-\omega_{-} m=0$. For fixed frequency scattering, this means that the reflection coefficient $\Re$ (i.e. the fixed frequency scattering operator from $\mathcal{H}_{R}$ to $\mathcal{C H}_{R}$ ) has a pole at $\omega-\omega_{-} m=0$ such that $\Re$ is of the form

$$
\mathfrak{R}=\frac{\mathfrak{r}(\omega, m, \ell)}{\omega-\omega \_m},
$$

where $\mathfrak{r}\left(\omega=\omega_{-} m, m, \ell\right) \neq 0$.

There is a natural solution $\psi$ defined in the black hole interior by continuing each quasinormal mode appearing in (1.17) into the interior. This solution is again smooth across $\mathcal{H}_{R}$ and thus can be view as a solution arising from smooth data on a spacelike hypersurface which coincides with $\left\{t^{*}=0\right\}$ on the exterior. Let us assume for a moment that the fixed frequency scattering theory also carries over to complex frequencies and that we can analytically continue the reflection coefficient $\mathfrak{R}$ to the complex plane. We then expect that the continued solution $\psi$ at the Cauchy horizon can be obtained by multiplying each individual coefficient $\psi \Gamma_{\mathcal{H}}$ as in (1.18) with the reflection coefficient $\mathfrak{R}\left(\omega_{m \ell n}, m, \ell\right)$. Moreover, neglecting $\mathfrak{r}\left(\omega_{m \ell n}, m, \ell\right)$ which is expected to be suitably bounded from below and above, and taking the $L^{2}\left(\mathbb{S}^{2}\right)$-norm of the $\{u=$ const $\}$-spheres on the Cauchy horizon $\mathcal{C H}_{R}$, formally yields

$$
\| \psi\left\lceil\mathcal{C H} \|_{L^{2}\left(\mathbb{S}^{2}\right)}^{2} \sim \sum_{m \in \mathbb{Z}} \sum_{\ell \geq|m|} \sum_{n=1}^{N(m, \ell)} \frac{|a(m, \ell, n)|^{2}}{\left|\omega_{m \ell n}-\omega_{-} m\right|^{2}},\right.
$$

where we recall that $a(m, \ell, n)$ decay exponentially as in (1.20).

In order to resolve Conjecture 3, we have to determine whether for all coefficients $a(m, \ell, n)$ satisfying (1.20), the sum (1.22) remains uniformly bounded, or whether, for some choice of $a(m, \ell, n)$ satisfying (1.20), this sum is infinite. Before we address this issue in the next paragraph, we refer to Fig. 6 for an illustration of the main difference between the behavior of linear scalar perturbations on Reissner-Nordström-AdS and Kerr-AdS.

\subsection{Small divisors and relation to Diophantine approximation}

The convergence of (1.22) is an example par excellence of a small divisors problem. Indeed, if $\left|\omega_{m \ell n}-\omega_{-} m\right|$ is exponentially small in $m, \ell, n$, the sum in 

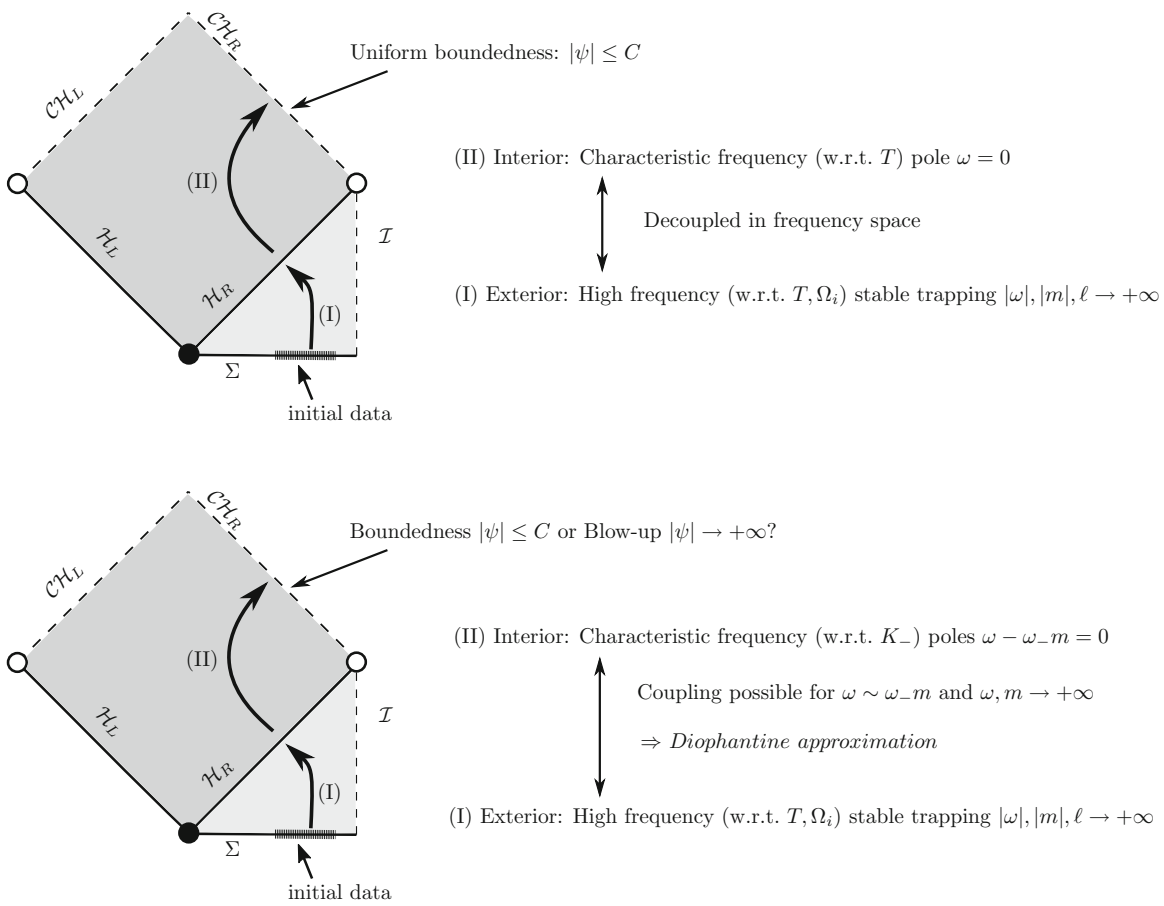

Fig. 6 Reissner-Nordström-AdS (top): High frequency stably trapped perturbations are decoupled in frequency space from interior scattering pole at characteristic frequency $\omega=0$ (w.r.t. $T$ ). Kerr-AdS (bottom): High frequency stably trapped perturbations couple in frequency space to interior scattering poles at characteristic frequency $\omega-\omega_{-} m=0$ (w.r.t. $K_{-}$)

(1.22) is infinite for suitable (in fact generic) $a(m, \ell, n)$ satisfying (1.20). More precisely, for the sum in (1.22) to be infinite for some choice of $a(m, \ell, n)$, in view of (1.20), it suffices that there exist infinitely many $(m, \ell, n)$ such that $\left|\omega_{m \ell n}-\omega_{-} m\right|$ decays exponentially. Thus, we conjecture blow-up if

$$
\left|\omega_{m \ell n}-\omega_{-} m\right| \leq c^{\prime} \exp (-C \ell) \text { for infinitely many admissible }(m, \ell, n),
$$

where $(m, \ell, n)$ are admissible if $m \in \mathbb{Z}, \ell \geq|m|, n=1, \ldots, N(m, \ell)$.

Conditions like (1.23) lie at the heart of Diophantine approximation. Indeed, semi-classical heuristics as in (1.16) suggest that $\operatorname{Re}\left(\omega_{m \ell n}\right)$ are uniformly distributed and we assume for a moment that $\operatorname{Re}\left(\omega_{m \ell n}\right)=\tilde{c}\left(\ell+\frac{n}{\ell}\right)$ for $n=0,1, \ldots, \ell$ for a constant $\tilde{c}=\tilde{c}(M, a, \Lambda)$ and that $\left|\operatorname{Im}\left(\omega_{m \ell n}\right)\right| \lesssim e^{-c \ell}$. For the sake of the purely heuristic argument assume also for a moment that the dimensionless constants $C$ as well as $c^{\prime} / \tilde{c}$ are actually $C=c^{\prime} / \tilde{c}=1$. Then, the ratio $r(\mathfrak{m}, \mathfrak{a}):=\frac{\omega_{-}}{\tilde{c}}$, which is dimensionless and only depends on 
the dimensionless black hole parameters $(\mathfrak{m}=M \sqrt{-\Lambda}, \mathfrak{a}=a \sqrt{-\Lambda})$, has to satisfy the non-Diophantine condition

$$
r(\mathfrak{m}, \mathfrak{a}) \in \mathscr{R}:=\left\{x \in \mathbb{R}:\left|\frac{\ell+\frac{n}{\ell}}{m}-x\right|<\exp (-\ell) \text { for } \infty \text {-many admissible }(m, \ell, n)\right\} .
$$

Thus, from our heuristic derivation, it is natural to conjecture that linear perturbations blow up at the Cauchy horizon of Kerr-AdS with mass $M=\mathfrak{m} / \sqrt{-\Lambda}$ and angular momentum $a=\mathfrak{a} / \sqrt{-\Lambda}$ if the ratio $r=r(\mathfrak{m}, \mathfrak{a})$ satisfies the nonDiophantine condition (1.24). At this point it worth emphasizing that the above arguments are merely heuristics and by no means can be turned into a proof easily. In particular, our proof does not use a quasinormal modes approach as the previous heuristics and the non-Diophantine condition (see already Sect. 5) is significantly more technical (refer also to the discussion later in Sect. 1.8).

The set $\mathscr{R} \mathrm{R}$ is Baire-generic and Lebesgue-exceptional. The set $\mathscr{R}$ can be written as a lim sup set as

$$
\mathscr{R}=\bigcap_{m_{0} \in \mathbb{N}} \bigcup_{|m| \geq m_{0}} \bigcup_{\ell \geq|m|} \bigcup_{0 \leq n \leq \ell}\left\{x \in \mathbb{R}:\left|\frac{\ell+\frac{n}{\ell}}{m}-x\right|<\exp (-\ell)\right\}
$$

It is a countable intersection of open and dense sets such that $\mathscr{R}$ is of second category in view of Baire's theorem [2]. Thus, the set $\mathscr{R}$ is generic from a topological point of view, which we refer to as Baire-generic. On the other hand, from a measure-theoretical point of view, the set $\mathscr{R}$ is exceptional. Indeed, an application of the Borel-Cantelli lemma shows that the Lebesgue measure of $\mathscr{R}$ vanishes. This is the easy part of the famous theorem by Khintchine [73] stating that for a decreasing function $\phi$, the set

$$
W[\phi]:=\left\{x \in \mathbb{R}:\left|x-\frac{p}{q}\right|<\frac{\phi(q)}{q} \text { for } \infty \text {-many rationals } \frac{p}{q}\right\}
$$

has full Lebesgue measure if and only if the sum $\sum_{q} \phi(q)$ diverges. Thus, $\mathscr{R}$ is Lebesgue-exceptional.

More refined measure: The Hausdorff and packing measures. This naturally leads us to consider the more refined versions of measure, the so-called Hausdorff and packing measures $H^{f}, P^{f}$ together with their associated dimensions $\operatorname{dim}_{H}, \operatorname{dim}_{P}$ (see Sect. 2.1). The Hausdorff and packing measure generalize the Lebesgue measure to non-integers. In a certain sense, they can be considered to be dual to each-other: The Hausdorff measure approximates and measures sets by a most economical covering, whereas the packing measure packs as many disjoint balls with centers inside the set. While for all sufficiently nice sets these notions agree, they indeed turn out to give different results in our context. 
We first consider the Hausdorff dimension. A version of the Borell-Cantelli lemma (more precisely the Hausdorff-Cantelli lemma) and using the natural cover for $\mathscr{R}$ shows that the set $\mathscr{R}$ is of Hausdorff dimension zero. This again can be seen as a consequence of a theorem going back to Jarník [67] and Besicovitch [8] which states the set $W[\phi]$ as in (1.26) has Hausdorff measure

$$
H^{s}(W[\phi])= \begin{cases}0 & \text { if } \sum_{q} q^{1-s} \psi^{s}(q)<\infty, \\ +\infty & \text { if } \sum_{q} q^{1-s} \psi^{s}(q)=\infty\end{cases}
$$

for $s \in(0,1)$. However, measuring also logarithmic scales, i.e. considering the Hausdorff measure $H^{f}$ for $f=\log ^{t}(r)$ for some $t>0$, it follows that the set $\mathscr{R}$ is of logarithmic generalized Hausdorff dimension. On the other hand, using the dual notion of packing dimension, it turns out that $\mathscr{R}$ has full packing dimension, a consequence of the fact that it is a set of second category (Baire-generic) [36].

Summary of properties of $\mathscr{R} \mathrm{R}$. To summarize, we obtain that

- $\mathscr{R}$ is Baire-generic,

- $\mathscr{R}$ is Lebesgue-exceptional,

- $\mathscr{R}$ has zero Hausdorff dimension $\operatorname{dim}_{H}(\mathscr{R})=0$,

- $\mathscr{R}$ is of logarithmic generalized Hausdorff dimension,

- $\mathscr{R}$ has full packing dimension $\operatorname{dim}_{P}(\mathscr{R})=1$.

The above heuristics will enter in our revised conjectures, Conjecture 5 and Conjecture 6, which transcend Conjecture 3 and Conjecture 4 for $\Lambda<0$. Before we turn to that in Sect. 1.5, we briefly discuss other aspects of PDEs and dynamical systems for which Diophantine approximation plays a crucial role.

Small divisors problems and Diophantine approximation in dynamical systems and PDEs. Most prominently, Diophantine approximation and the small divisors problem are intimately tied to the problem of the stability of the solar system [89] and more generally, the stability of Hamiltonian systems in classical mechanics. This stability problem was partially resolved with the celebrated KAM theorem $[1,74,88]$ which roughly states that Lebesgue-generic perturbations of integrable Hamiltonian systems lead to quasiperiodic orbits. The small divisors problem and Diophantine approximation are ubiquitous in modern mathematics and arise naturally in many other aspects of PDEs and dynamical systems. We refer to $[37,90]$ for a connection to wave equations with periodic boundary conditions and to the more general results in [50] as well as the monograph [101]. There is also a vast recent literature on the construction of (quasi-)periodic orbits to nonlinear wave equations; we refer to $[3,6,117]$, the overview article [5] and the monograph [7] and references therein for further details. Similar results have been obtained for the 
Schrödinger equation on the torus in $[35,68,75,116]$. Further applications of Diophantine approximation include the characterization of homeomorphisms on $\mathbb{S}^{1}$ by the Diophantine properties of their rotation numbers or analyzing the Lyapunov stability of vector fields, see the discussion in [77].

\subsection{Conjecture 5 and Conjecture 6 replace Conjecture 3 and Conjecture 4 for Kerr-AdS}

With the above heuristics in hand, we now transcend Conjecture 3 and Conjecture 4 for subextremal Kerr-AdS black holes with parameters below the Hawking-Reall bound in terms of the following two conjectures. We denote the set of all such parameters with $\mathscr{P}$, see already (2.9).

Conjecture 5 There exists a set $\mathscr{P}$ Blow-up $\subset \mathscr{P}$ of dimensionless Kerr-AdS parameters mass $\mathfrak{m}=M \sqrt{-\Lambda}$ and angular momentum $\mathfrak{a}=a \sqrt{-\Lambda}$ with the following properties

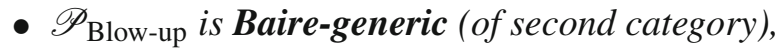

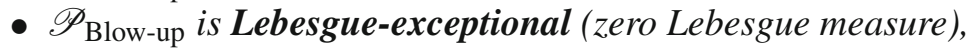

and such that for every Kerr-AdS black hole with mass $M=\mathfrak{m} / \sqrt{-\Lambda}$ and spe-

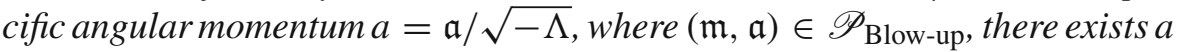
solution $\psi$ to (1.2), which arises from smooth and compactly supported initial data $\left(\psi_{0}, \psi_{1}\right)$ on a suitable spacelike hypersurface with Dirichlet boundary conditions at infinity, and which blows up

$$
\|\psi\|_{L^{2}\left(\mathbb{S}^{2}\right)}(u, r) \underset{r \rightarrow r_{-}}{\longrightarrow}+\infty
$$

at the Cauchy horizon for every $u \in \mathbb{R}$.

Remark 1.1 If there exist initial data $\left(\psi_{0}, \psi_{1}\right)$ leading to a solution $\psi$ which blows up as in (1.28), this then shows that initial data $\left(\tilde{\psi}_{0}, \tilde{\psi}_{1}\right)$ for which the arising solution does not blow up are exceptional in the sense that they obey the following co-dimension 1 property: The solution arising from the perturbed data $\left(\tilde{\psi}_{0}+c \psi_{0}, \tilde{\psi}_{1}+c \psi_{1}\right)$ blows up for each $c \in \mathbb{R} \backslash\{0\}$. This is analogous to the notion of genericity used by Christodoulou in his proof of weak cosmic censorship for the spherically symmetric Einstein-scalar-field system [14,15]. Thus, Conjecture 5 gives a formulation of Conjecture 3 . We note already that we will actually formulate in Remark 1.5 another more refined genericity condition for the set of initial data leading to solutions which blow up as in (1.28).

Remark 1.2 Note that in Conjecture 5 we have replaced the statement of blowup in amplitude from Conjecture 3 with a statement about the blow-up of 
the $L^{2}\left(\mathbb{S}^{2}\right)$-norm on the sphere. Indeed, the blow-up of the $L^{2}\left(\mathbb{S}^{2}\right)$-norm in Conjecture 5, if true, implies that $\|\psi\|_{L^{\infty}\left(\mathbb{S}^{2}\right)}(u, r) \rightarrow+\infty$ as $r \rightarrow r_{-}$. In this sense, if Conjecture 5 is true, the amplitude also blows up. It is however an interesting and open question whether one may actually replace the $L^{\infty}\left(\mathbb{S}^{2}\right)$ blow-up statement in (1.28) with the pointwise blow-up

$$
\lim _{r \rightarrow r_{-}}\left|\psi\left(u, r, \theta, \phi_{-}^{*}\right)\right| \rightarrow+\infty
$$

for every $\left(\theta, \phi_{-}^{*}\right) \in \mathbb{S}^{2}$. One may even speculate about the geometry of the set of $\left(\theta, \phi_{-}^{*}\right) \in \mathbb{S}^{2}$ for which pointwise blow-up holds. It appears that ultimately one has to quantitatively understand the nodal domains associated to the generalized spheroidal harmonics $S_{m \ell}\left(a \omega_{-} m, \cos \theta\right)$ at the interior scattering poles.

Remark 1.3 Moreover, we conjecture that the set $\mathscr{P}_{\text {Blow-up }}$ has

- Hausdorff dimension $\operatorname{dim}_{H}\left(\mathscr{P}_{\text {Blow-up }}\right)=1$,

- generalized Hausdorff dimension $\operatorname{dim}_{g H}\left(\mathscr{P}_{\text {Blow-up }}\right)=1+\log$,

- full packing $\operatorname{dimension}_{\operatorname{dim}}\left(\mathscr{P}_{\text {Blow-up }}\right)=2$.

Moreover, in view of our discussion we additionally conjecture

Conjecture 6 (A) There exists a set $\mathscr{P}_{\text {Bounded }} \subset \mathscr{P}$ of dimensionless KerrAdS parameters mass $\mathfrak{m}=M \sqrt{-\Lambda}$ and angular momentum $\mathfrak{a}=a \sqrt{-\Lambda}$ with the following properties

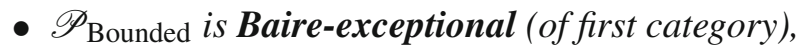

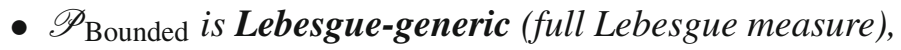

and such that for every Kerr-AdS black hole with mass $M=\mathfrak{m} / \sqrt{-\Lambda}$ and specific angular momentum $a=\mathfrak{a} / \sqrt{-\Lambda}$, where $(\mathfrak{m}, \mathfrak{a}) \in \mathscr{P}_{\text {Bounded, all }}$ solutions $\psi$ to (1.2), which arise from smooth and compactly supported initial data $\left(\psi_{0}, \psi_{1}\right)$ on a spacelike hypersurfaces with Dirichlet boundary conditions at infinity, remain uniformly bounded

$$
|\psi| \leq C(\mathfrak{m}, \mathfrak{a}) D\left(\psi_{0}, \psi_{1}\right)
$$

at the Cauchy horizon. Here, $D\left(\psi_{0}, \psi_{1}\right)$ is a (higher-order) energy of the initial data and $C(\mathfrak{m}, \mathfrak{a})$ is a constant depending on $\mathfrak{m}$ and $\mathfrak{a}$.

(B) For all Kerr-AdS black holes with parameters in $\mathscr{P}$, there exists a solution $\psi$ to (1.2), which arises from smooth and compactly supported initial data on a spacelike hypersurfaces with Dirichlet boundary conditions at infinity and which blows up in energy

$$
\|\psi\|_{H_{\mathrm{loc}}^{1}}=+\infty
$$


at the Cauchy horizon.

Remark 1.4 In view of Conjecture 5, we expect that the constant $C(\mathfrak{m}, \mathfrak{a})$ appearing in (1.30) to be unbounded on any open set of parameters in the sense that

$$
\sup _{(\mathfrak{m}, \mathfrak{a}) \in \mathcal{U} \cap \mathscr{P}_{\text {Bounded }}} C(\mathfrak{m}, \mathfrak{a})=+\infty
$$

for any non-empty open $\mathcal{U} \subset \mathscr{P}$.

More general boundary conditions and Klein-Gordon masses. The above conjectures are both stated for Dirichlet conditions at infinity. Neumann conditions are also natural to consider and indeed well-posedness was proved in $[66,118]$. For Neumann conditions we also expect the same behavior as for the case of Dirichlet boundary conditions. For other more general conditions, it may be the case that linear waves grow exponentially (as for suitable Robin boundary conditions [66]) or on the other hand even decay superpolynomially as is the case for purely outgoing conditions [62]. For even more general boundary conditions, even well-posedness may be open.

In this paper we have focused on scalar perturbations satisfying (1.2). In particular, the choice of the Klein-Gordon mass parameter $\mu=\frac{2}{3} \Lambda$ ("conformal coupling") is the most natural as it arises from the linear scalar analog of (1.1) and also remains regular at infinity. However, in certain situations it may also be interesting to consider more general Klein-Gordon masses $\mu$ satisfying the Breitenlohner-Friedman [12] bound $\mu>\frac{3}{4} \Lambda$. We also expect Conjecture 5 and Conjecture 6 to hold for Klein-Gordon masses for which the exterior is linearly stable, i.e. for $\mu>\frac{3}{4} \Lambda$ in the case of Dirichlet boundary conditions, and for $\frac{3}{4} \Lambda<\mu<\frac{5}{12} \Lambda$ together with additional assumptions in the case of Neumann boundary conditions [66].

Regularity of the initial data. We stated Conjecture 5 and Conjecture 6 for smooth $\left(C^{\infty}\right)$ initial data. One can also consider classes of initial data which are more regular (e.g. Gevrey or analytic) or less regular (e.g. Sobolev). From our heuristics, we expect that the analogs of Conjecture 5 and Conjecture 6 remain valid both for rougher data in some suitably weighted Sobolev space (see [63]) and more regular data of Gevrey regularity with index $\sigma>1$ and analytic data $(\sigma=1)$. Only in the exceptional and most regular case of initial data with Gevrey regularity $\sigma<1$ (note that this is more regular than analytic) in the angular direction $\partial_{\phi}$, we expect the analog of Conjecture 5 to break down. In particular, for axisymmetric data (or data supported only on finitely many azimuthal modes $m$ ), we expect the arising solution to remain uniformly bounded at the Cauchy horizon for all parameters in $\mathscr{P}$. 


\subsection{Theorem 1: Conjecture 5 is true}

Our main result is the following resolution of Conjecture 5.

\section{Theorem 1 Conjecture 5 is true.}

The proof of Theorem 1 will be given in Sect. 9 .

Remark 1.5 In the proof of Theorem 1 we will not only construct a single solution which blows up leading to genericity of initial data as in Remark 1.1 but we will actually obtain what is perhaps a more satisfying genericity condition on the initial data which are smooth and of compact support. We formulate this condition in Corollary 1 in Sect. 9.

Remark 1.6 We also prove in Sect. 9 the statement about the packing dimen-

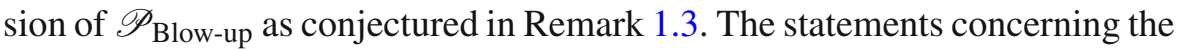
Hausdorff dimension, however, remain open.

Remark 1.7 In principle, our proof is expected to also apply to Neumann boundary conditions as well as to more general Klein-Gordon masses satisfying the Breitenlohner-Friedman bound [12] as discussed at the end of Sect. 1.5.

\subsection{Outlook on Conjecture 6}

We also expect that our methods provide a possible framework for the resolution of Conjecture 6.

First, note that the blow-up statement of Theorem 1 is strictly stronger than the $H_{\text {loc }}^{1}$ blow-up conjectured in Conjecture 6(B). Thus, Theorem 1 shows that Conjecture 6(B) is true for black hole parameters in the set $\mathscr{P}_{\text {Blow-up. For }}$ parameters not contained in $\mathscr{P}_{\text {Blow-up, we expect that a quasinormal mode }}$ which decays at a sufficiently slow exponential decay rate compared to the surface gravity of the Cauchy horizon will blow up in energy at the Cauchy horizon. This would show Conjecture 6(B). Towards Conjecture 6(A), we note that our proof, particularly formula (9.6) of Proposition 9.1, reveals the main obstruction for boundedness. Together with the methods used in [70] for the Reissner-Nordström-AdS case, this can serve as a starting point for a resolution of Conjecture 6(A).

\subsection{Turning the heuristics of Section 1.4 into a proof of Theorem 1}

We will now outline how we turn our heuristics of Sect. 1.4 into a proof of Conjecture 5, i.e. Theorem 1. 
We are interested in constructing a solution of (1.2), arising from smooth and compactly supported initial data, which blows up as in (1.28), if the dimensionless parameters $\mathfrak{m}, \mathfrak{a}$ satisfy a certain non-Diophantine condition.

We remark that unlike in our heuristic discussion, we will not make use of quasinormal modes and the frequency analysis will be purely based on the real axis with $\omega \in \mathbb{R}$. Indeed, our approach can be interpreted as replacing quasinormal modes with quasimodes. This will also manifest itself in the fact that the roles of $\frac{1}{\mathfrak{W}\left[u_{\mathcal{H}^{+}}, u_{\infty}\right](\omega, m, \ell)}$ and $\frac{1}{\omega-\omega_{-} m}$ will be changed: In the heuristic analysis, we considered the quasinormal mode frequencies $\omega_{m \ell n}$ which are (complex) roots of the Wronskian $\mathfrak{W}\left[u_{\mathcal{H}^{+}}, u_{\infty}\right]$ and the small divisors came from $\frac{1}{\omega_{m} \ell_{n}-\omega_{-} m}$. In the actual proof of Theorem 1, we will instead consider the real frequencies $\omega=\omega_{-} m$ (i.e. the roots of $\omega-\omega_{-} m=0$ ) and as we will see, the small divisors will then appear from the Wronskian evaluated at the characteristic frequency $\frac{1}{\mathfrak{W}\left[u_{\mathcal{H}^{+}}, u_{\infty}\right]\left(\omega=\omega_{-} m, m, \ell\right)}$. Note that the divisor $\left|\mathfrak{W}\left[u_{\mathcal{H}^{+}}, u_{\infty}\right]\left(\omega=\omega_{-} m, m, \ell\right)\right|$ is small exactly if there exists a quasimode with frequency $\left(\omega=\omega_{-} m, m, \ell\right)$. In view of the distribution of the quasimode frequencies discussed in Sect. 1.2, this will lead to a (generalized) non-Diophantine condition which we will address in more detail further below.

Initial data and exterior analysis (Sect. 6 and Sect. 7). We begin our discussion with our choice of initial data. In Sect. 6 we will carefully impose smooth and compactly supported initial data $\Psi_{0}, \Psi_{1} \in C_{c}^{\infty}\left(\Sigma_{0}\right)$ for (1.2) on the spacelike hypersurface $\Sigma_{0}=\{t=0\}$ which—with foresight-will be chosen to satisfy

$$
\left|G\left(\Psi_{0}, \Psi_{1}, m_{i}, \ell_{i}\right)\right| \gtrsim e^{-m_{i}^{\frac{1}{3}}}
$$

for suitable infinite sequences $m_{i}, \ell_{i}$, where

$$
\begin{aligned}
G\left(\Psi_{0}, \Psi_{1}, m, \ell\right):= & \int_{\Sigma_{0}} u_{\infty}\left(r, \omega=\omega_{-} m, m, \ell\right) \\
& \times S_{m \ell}\left(a \omega=a \omega_{-} m, \cos \theta\right) e^{-i m \phi} \\
& \times H\left(\Psi_{0}, \Psi_{1}\right)\left(\omega_{-} m, r, \theta, \phi\right) \operatorname{dvol}_{\Sigma_{0}},
\end{aligned}
$$

and

$H\left(\Psi_{0}, \Psi_{1}\right)(\omega, r, \theta, \phi)=\frac{\Sigma}{r^{2} \sqrt{r^{2}+a^{2}}}\left(-\sqrt{-g^{t t}} \Psi_{1}-i \omega g^{t t} \Psi_{0}+g^{t \phi} \partial_{\phi} \Psi_{0}\right)$.

We also recall that $u_{\infty}(r, \omega, m, \ell)$ is the solution to the radial o.d.e. adapted to the Dirichlet boundary condition at $\mathcal{I}$. 
Complementing the data with vanishing data on $\mathcal{H}_{L} \cup \mathcal{B}_{\mathcal{H}}$, the data $\Psi_{0}, \Psi_{1}$ define a solution on the black hole interior. Different from our heuristic discussion with quasinormal modes (i.e. fixed frequency solutions) in Sect. 1.4, in the present case, we do need to consider the analog of a "full" scattering operator $\mathfrak{S}_{\mathcal{H}_{R} \rightarrow \mathcal{C H}_{R}}$ from the event horizon to the Cauchy horizon which would be of the form

$$
\mathfrak{S}_{\mathcal{H}_{R} \rightarrow \mathcal{C H}_{R}}=\mathcal{F}_{\mathcal{C H}}^{-1} \circ \mathfrak{R}(\omega, m, \ell) \circ \mathcal{F}_{\mathcal{H}}=\mathcal{F}_{\mathcal{C H}}^{-1} \circ \frac{\mathfrak{r}(\omega, m, \ell)}{\omega-\omega_{-} m} \circ \mathcal{F}_{\mathcal{H}},
$$

where $\mathcal{F}_{\mathcal{H}}$ and $\mathcal{F}_{\mathcal{C H}}$ represent (generalized) Fourier transforms along the event and Cauchy horizon, respectively. Thus, from the exterior, we need to determine the generalized Fourier transform $\mathcal{F}_{\mathcal{H}}[\psi\lceil\mathcal{H}]$ along the event horizon. Such a characterization in terms of the chosen initial data from above is the content of Sect. 7. While in the actual proof (see already Proposition 7.1), we will use a suitably truncated generalized Fourier transform, we may formally think of $\mathcal{F}_{\mathcal{H}}[\psi\lceil\mathcal{H}]$ as having the form

$$
\begin{aligned}
\mathcal{F}_{\mathcal{H}}[\psi\lceil\mathcal{H}](\omega, m, \ell) & \\
\sim & \frac{\int_{\Sigma_{0}} u_{\infty}(r, \omega, m, \ell) S_{m \ell}(a \omega, \cos \theta) e^{-i m \phi} H\left(\Psi_{0}, \Psi_{1}\right)(\omega, r, \theta, \phi) \mathrm{dvol}_{\Sigma_{0}}}{\mathfrak{W}\left[u_{\mathcal{H}^{+}}, u_{\infty}\right](\omega, m, \ell)} .
\end{aligned}
$$

We already remark that a consequence of the smoothness of the initial data is that $G\left(\Psi_{0}, \Psi_{1}, m, \ell\right)$ decays superpolynomially in $m$ and $\ell$, cf. (6.26) in Sect. 6.

Interior analysis (Sects. 8, 9 and some of Sect. 3. Turning to the interior analysis, we recall from our heuristic discussion in Sect. 1.3 that the analog of the scattering operator (1.36) from the event to the Cauchy horizon has poles at the characteristic frequencies $\omega-\omega_{-} m=0$ with respect to $K_{-}$. In our heuristic discussion in Sect. 1.4 based on quasinormal modes and fixed frequency scattering, these poles formally lead to (1.22). In the actual proof, based on frequency analysis on the real axis, the scattering poles become evident in formula (9.6) stated in Proposition 9.1 which roughly translates to the statement that, as $r \rightarrow r_{-}$, we have

$$
\begin{aligned}
& \left\|\psi\left(u_{0}, r\right)\right\|_{L^{2}\left(\mathbb{S}^{2}\right)}^{2} \sim \sum_{m \ell}\left|\mathfrak{r}\left(\omega=\omega_{-} m, m, \ell\right)\right|^{2}\left|\mathcal{F}_{\mathcal{H}}[\psi \mid \mathcal{H}]\left(\omega=\omega_{-} m, m, \ell\right)\right|^{2} \\
& \quad+\operatorname{Err}(D)
\end{aligned}
$$

where $\operatorname{Err}(D)$ is uniformly bounded by an (higher order) energy of the initial data. (Note that in the actual statement of Proposition 9.1, the Fourier transform 


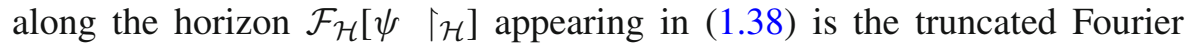
transform $a_{\mathcal{H}}^{R_{n}}\left(\omega=\omega_{-} m, m, \ell\right)=\mathcal{F}_{\mathcal{H}}\left[\psi\left\lceil_{\mathcal{H}} \nVdash_{v \leq R_{n}}\right]\right.$ for $R_{n}=2 r_{n}^{*}-u_{0}+\tilde{c}$. $)$

Both the proof and the use of (1.38) lie at the heart of the proof of Theorem 1. The proof of (1.38) is technical and combines physical space methods with techniques from harmonic analysis. One of the key technical steps (see Proposition 3.3 in Sect. 3) is a quantitative bound (see already (3.72)) on the derivative of the generalized spheroidal harmonics

$$
\sup _{\left|a \omega-a \omega_{-} m\right|<\frac{1}{m}}\left\|\partial_{\omega} S_{m \ell}(a \omega)\right\|_{L^{2}}^{2} \lesssim m
$$

near the interior scattering poles $\omega=\omega_{-} m$. The proof of (1.39) relies on uniform bounds (in $m, \ell$ and $\omega \approx \omega_{-} m$ ) on the resolvent of the associated singular Sturm-Liouville operator, see the discussion in Sect. 3.3. These bounds are shown by constructing and estimating the integral kernel of the resolvent using suitable approximations with parabolic cylinder functions and Airy functions. For solutions of the radial o.d.e. in the interior, the analogous resolvent bounds are shown in Sect. 8.1. Their proofs rely on semi-classical approximations and estimates on Volterra integral equations.

Further ingredients to control the error term $\operatorname{Err}(D)$ in (1.38) are uniform bounds on the transmission and reflection coefficients $\mathfrak{T}(\omega, m, \ell)$ and $\mathfrak{R}(\omega, m, \ell)$ for frequencies which are bounded away from the characteristic frequency $\omega=\omega_{-} m$.

Combining the exterior with the interior: Occurrence of small divisors and the proof of Theorem 1 Sect. 9 . We will now connect the exterior analysis to the interior. In particular, formally plugging (1.37) into (1.38) and noting that

$$
\mathcal{F}_{\mathcal{H}}\left[\psi\lceil\mathcal{H}]\left(\omega=\omega_{-} m, m, \ell\right) \sim \frac{G\left(\Psi_{0}, \Psi_{1}, m, \ell\right)}{\mathfrak{W}\left[u_{\mathcal{H}^{+}}, u_{\infty}\right]\left(\omega=\omega_{-} m, m, \ell\right)},\right.
$$

yields in the limit $r \rightarrow r_{-}$that

$$
\left\|\psi\left(u_{0}, r\right)\right\|_{L^{2}\left(\mathbb{S}^{2}\right)}^{2} \sim \sum_{m \ell} \frac{|m|^{2}\left|G\left(\Psi_{0}, \Psi_{1}, m, \ell\right)\right|^{2}}{\left|\mathfrak{W}\left[u_{\mathcal{H}^{+}}, u_{\infty}\right]\left(\omega=\omega_{-} m, m, \ell\right)\right|^{2}}+\operatorname{Err}(D),
$$

where we also used that the (renormalized) reflection coefficient satisfies $\left|\mathfrak{r}\left(\omega=\omega_{-} m, m, \ell\right)\right| \sim|m|$ which we will show in Lemma 8.7. Also recall from before that the error term $|\operatorname{Err}(D)|$ is shown to remain uniformly bounded as $r \rightarrow r_{-}$. Remark that in the actual proof we will not quite show (1.41) but rather obtain (9.43) which corresponds to (1.41) in a certain limiting sense. We also recall from the discussion of the exterior analysis that the 
term $|m|^{2}\left|G\left(\Psi_{0}, \Psi_{1}, m, \ell\right)\right|^{2}$ which appears in the sum of (1.41) as the numerator, decays superpolynomially in $m$ and $\ell$. Thus, at least formally, in order to show blow-up for (1.41), it is necessary that small divisors in (1.41) occur infinitely often, i.e. that the Wronskian evaluated at the interior scattering poles $\mathfrak{W}\left[u_{\mathcal{H}^{+}}, u_{\infty}\right]\left(\omega=\omega_{-} m, m, \ell\right)$ (cf. Sect. 1.2) decays (at least) superpolynomially for infinitely many $(m, \ell)$. In our proof, we will actually require from the black hole parameters $\mathfrak{m}, \mathfrak{a}$ that this Wronskian decays exponentially

$$
\left|\mathfrak{W}\left[u_{\mathcal{H}^{+}}, u_{\infty}\right]\left(\omega=\omega_{-} m, m, \ell\right)\right| \leq e^{-m^{\frac{1}{2}}} \text { for infinitely many }(m, \ell) .
$$

Before we address the validity of (1.42), we will assume for a moment that indeed the black hole parameters $\mathfrak{m}, \mathfrak{a}$ are such that (1.42) holds true. Then, explicitly choose that the subsequences $m_{i}$ and $\ell_{i}$ in (1.33) coincide with the infinite sequences which fulfill (1.42). Then, we formally obtain the blow-up result of Theorem 1 as

$$
\begin{aligned}
\lim _{r \rightarrow r_{-}}\left\|\psi\left(u_{0}, r\right)\right\|_{L^{2}\left(\mathbb{S}^{2}\right)}^{2} & \sim \sum_{i \in \mathbb{N}}\left|m_{i}\right|^{2} \frac{\left|G\left(\Psi_{0}, \Psi_{1}, m_{i}, \ell_{i}\right)\right|^{2}}{\left|\mathfrak{W}\left[u_{\mathcal{H}^{+}}, u_{\infty}\right]\left(\omega=\omega_{-} m_{i}, m_{i}, \ell_{i}\right)\right|^{2}} \\
& \sim \sum_{i \in \mathbb{N}}\left|m_{i}\right|^{2} \frac{\left.e^{-m_{i}^{\frac{1}{3}}}\right|^{2}}{\left|e^{-m_{i}^{\frac{1}{2}}}\right|^{2}}=+\infty
\end{aligned}
$$

Similarly to the remark before, in reality, (1.43) holds true only in a certain limiting sense, cf. (9.43)-(9.45) of Sect. 9. Already from (1.43) and (1.42) we obtain the following genericity condition

$$
\sum_{i \in \mathbb{N}}\left|m_{i} e^{m_{i}^{\frac{1}{2}}}\right|^{2}\left|G\left(\Psi_{0}, \Psi_{1}, m_{i}, \ell_{i}\right)\right|^{2}=+\infty
$$

on the initial data leading to blow-up. This will be formulated as Corollary 1 in Sect. 9.

The non-Diophantine condition and its relation to quasimodes (Sect. 4, Sect. 5, and some of Sect. 3. Finally, this leaves us to address the question of whether the small divisors in (1.41) actually appear infinitely often, more precisely, whether (1.42) holds true. The condition (1.42) constitutes a generalized non-Diophantine condition on the black hole parameters $\mathfrak{m}$, $\mathfrak{a}$ in view of its relation to the (discrete) Bohr-Sommerfeld quantization conditions from our heuristic discussion Sect. 1.2. In our actual proof, the non-Diophantine condition which we define in Definition 5.3 in Sect. 5 is more technical than (1.42), though (1.42) should be considered as its key property. We denote the set of dimensionless black hole parameters $\mathfrak{m}, \mathfrak{a}$ which satisfy the condition 


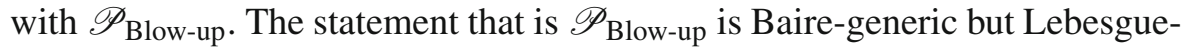
exceptional is the content of Sects. 5.2 and 5.3, respectively. Both proofs crucially rely on estimates developed in Sect. 4.

Connecting to the discussion of quasimodes before, we note that the nonDiophantine condition of (1.42) can be interpreted as the statement that there exist infinitely many quasimodes with frequency $\omega=\omega_{-} m$. This also implies that there exist infinitely many quasinormal modes with (complex) frequencies $\omega$ exponentially close to $\omega=\omega_{-} m$. However, note that quasimodes are more robust to perturbations in the sense that if $\omega, m, \ell$ are frequencies of a quasimode, there exists a (exponentially small) neighborhood of $\omega$ such that for each $\tilde{\omega}$ in that neighborhood, the frequencies $\tilde{\omega}, m, \ell$ would also describe a quasimode. It is also this robustness which is a key advantage of quasimodes over an approach based on quasinormal modes as in the heuristic discussion in Sect. 1.4.

\subsection{Outline of the paper}

In Sect. 2 we set up the Kerr-AdS spacetime, recall the well-posedness of (1.2) and the decay statement for solutions on the exterior. We also introduce Carter's separation of variables. Section 3 is devoted to the analysis of the angular o.d.e. In Sect. 4 we analyze the radial o.d.e. on the exterior and introduce suitable solutions of the radial o.d.e. associated to trapping at the interior scattering poles $\omega=\omega_{-} m$. With the estimates from Sect. 4 in hand, we define the set

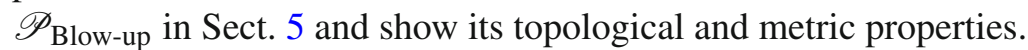

Then, for arbitrary but fixed parameters $\mathfrak{p} \in \mathscr{P}_{\text {Blow-up we define suitable }}$ compactly supported and smooth initial data in Sect. 6. In Sect. 7 we treat the exterior problem and conclude with a representation formula of the solution along the horizon in terms of the initial data. In Sect. 8 we first show suitable estimates for solutions of the radial o.d.e. in the interior before we finally conclude the paper with the proof of Theorem 1 in Sect. 9.

\section{Preliminaries}

\subsection{Fractal measures and dimensions}

\subsubsection{Hausdorff and Packing measures}

We begin by introducing the Hausdorff and packing measure. We refer to the monograph [36] for a more detailed discussion. For an increasing dimension function $f:[0, \infty) \rightarrow[0, \infty)$ we define the Hausdorff measure $H^{f}(A)$ of a set $A$ as 


$$
H^{f}(A):=\sup _{\delta>0} H_{\delta}^{f}(A)
$$

where

$H_{\delta}^{f}(A):=\inf \left\{\sum_{i=1}^{\infty} f\left(\operatorname{diam}\left(U_{i}\right)\right):\left\{U_{i}\right\}_{i=1}^{\infty}\right.$ countable cover of $\left.A, \operatorname{diam}\left(U_{i}\right) \leq \delta\right\}$.

If $f(r)=r^{s}$, we write $H^{s}=H^{r^{s}}$ and for $s \in \mathbb{N}$, the measure $H^{s}$ reduces to the Lebesgue measure up to some normalization. While the Hausdorff measure quantifies the size of a set by approximation it from outside via efficient coverings, we also recall the dual notation: The packing measure quantifies the size of sets by placing as many disjoint balls with centers contained in the set. Again, for a dimension function $f$, we define the pre-measure

$$
\begin{aligned}
P_{0}^{f}(A):= & \limsup _{\delta \rightarrow 0}\left\{\sum_{i=1}^{\infty} f\left(\operatorname{diam}\left(B_{i}\right)\right):\left\{B_{i}\right\}_{i=1}^{\infty}\right. \text { collection of closed, } \\
& \text { pairwise disjoint balls with } \left.\operatorname{diam}\left(B_{i}\right) \leq \delta \text { and centers in } A\right\}
\end{aligned}
$$

and finally the packing measure as

$$
P^{f}(A):=\inf \left\{\sum_{i=1}^{\infty} P_{0}^{f}\left(A_{i}\right): A \subset \bigcup_{i=1}^{\infty} A_{i}\right\}
$$

\subsubsection{Hausdorff and Packing dimensions}

For $f(r)=r^{s}$ Hausdorff and Packing dimensions $\operatorname{dim}_{H}$ and $\operatorname{dim}_{P}$ are now characterized as the jump value, where the respective measure jumps from 0 to $\infty$, more precisely

$$
\operatorname{dim}_{H}(A)=\sup \left\{s: H^{s}(A)=0\right\}, \quad \operatorname{dim}_{P}(A)=\sup \left\{s: P^{s}(A)=0\right\} .
$$

We also say that a set $A$ has generalized Hausdorff $\operatorname{dimension}^{\operatorname{dim}_{g H}(A)=}$ $s+\log$ if the jump appears for the dimension function $f(r)=r^{s} \log ^{t}(r)$ for some $t>0$. 


\subsection{Kerr-AdS spacetime}

\subsubsection{Parameter space}

We let the value of the cosmological constant $\Lambda<0$ be fixed throughout the paper. For convenience and as it is convention, we re-parametrize the cosmological constant by the AdS radius

$$
l:=\sqrt{\frac{-3}{\Lambda}}
$$

We consider Kerr-AdS black holes which are parameterized by their mass $M>0$ and their specific angular momentum $a \neq 0$. Moreover, without loss of generality we will only consider $a>0$ and require $0<a<l$ for the spacetime to be regular. For $M>0,0<a<l$, we consider the polynomial

$$
\Delta(r):=\left(a^{2}+r^{2}\right)\left(1+\frac{r^{2}}{l^{2}}\right)-2 M r .
$$

We are interested in spacetimes without naked singularities. To ensure this, we define a parameter tuple $(M, a) \in \mathbb{R}_{>0}^{2}$ to be non-degenerate if $0<a<l$ and $\Delta(r)$ defined in (2.7) has two real roots satisfying $0<r_{-}<r_{+}$. Finally, to exclude growing mode solutions (see [33]) we assume the Hawking-Reall (non-superradiant) bound

$$
r_{+}^{2}>a l
$$

This leads us to the definition of the dimensionless black hole parameter space

$$
\mathscr{P}:=\left\{(\mathfrak{m}, \mathfrak{a}) \in \mathbb{R}_{>0}^{2}:(M, a):=(\mathfrak{m} l / \sqrt{3}, \mathfrak{a} l / \sqrt{3}) \text { is non-degenerate and } r_{+}^{2}>a l\right\} .
$$

Note that in view of (2.6), we have $M=\mathfrak{m} / \sqrt{-\Lambda}=\mathfrak{m} l / \sqrt{3}$ and $a=$ $\mathfrak{a} / \sqrt{-\Lambda}=\mathfrak{a} l / \sqrt{3}$. On the parameter space $\mathscr{P}$, we will also use the global coordinates $(\vartheta, \mathfrak{a})$, where

$$
\vartheta=\vartheta(\mathfrak{a}, \mathfrak{m}):=\frac{1-a^{2} / l^{2}}{1+r_{-}^{2} / a^{2}}
$$

(Note that $\vartheta=a \omega_{-}$, where $\omega_{-}$is defined in (2.16) below.) Thus, for each $\mathfrak{a}$, there exists an interval $\left(\vartheta_{1}(\mathfrak{a}), \vartheta_{2}(\mathfrak{a})\right)$ and a smooth embedding 
$\left(\vartheta_{1}(\mathfrak{a}), \vartheta_{2}(\mathfrak{a})\right) \rightarrow \mathscr{P}, \vartheta \mapsto(\mathfrak{m}(\vartheta), \mathfrak{a})$ which also depends smoothly on $\mathfrak{a}$. We define the vector field $\Gamma$ on $\mathscr{P}$ by

$$
\Gamma:=\frac{\partial}{\partial \vartheta}
$$

in coordinates $(\vartheta, \mathfrak{a})$. We define $\Phi_{\tau}^{\Gamma}$ as the flow generated by $\Gamma$.

Finally, remark that $\mathscr{P}$ is a Baire space as a (non-empty) open subset of $\mathbb{R}^{2}$. In particular, this allows us to speak about the notion of Baire-exceptional and Baire-generic subsets. Recall that a subset is Baire-exceptional if it is a countable union of nowhere dense sets and a subset is called Baire-generic if it is a countable intersection of open and dense sets. Note that if a subset is Baire-generic then its complement is Baire-exceptional and vice versa. Finally, in a Baire space every Baire-generic subset is dense.

\subsubsection{Kerr-AdS spacetime}

Fixed manifold. We begin by constructing the Kerr-AdS spacetime. We define the exterior region $\mathcal{R}$ and the black hole interior $\mathcal{B}$ as smooth four dimensional manifolds diffeomorphic to $\mathbb{R}^{2} \times \mathbb{S}^{2}$. On $\mathcal{R}$ and on $\mathcal{B}$ we assume to have global (up to the well-known degeneracy on $\mathbb{S}^{2}$ ) coordinate charts

$$
\begin{aligned}
& \left(t_{\mathcal{R}}, r_{\mathcal{R}}, \theta_{\mathcal{R}}, \phi_{\mathcal{R}}\right) \in \mathbb{R} \times\left(r_{+}, \infty\right) \times \mathbb{S}^{2}, \\
& \left(t_{\mathcal{B}}, r_{\mathcal{B}}, \theta_{\mathcal{B}}, \phi_{\mathcal{B}}\right) \in \mathbb{R} \times\left(r_{-}, r_{+}\right) \times \mathbb{S}^{2} .
\end{aligned}
$$

These coordinates $(t, r, \phi, \theta)$ are called Boyer-Lindquist coordinates. If it is clear from the context which coordinates are being used, we will omit their subscripts throughout the chapter.

The Kerr-AdS metric. For $(\mathfrak{m}, \mathfrak{a}) \in \mathscr{P}$ and $M=\mathfrak{m} l / \sqrt{3}$ and $a=\mathfrak{a} l / \sqrt{3}$, we define the Kerr-AdS metric on $\mathcal{R}$ and $\mathcal{B}$ in terms of the Boyer-Lindquist coordinates as

$$
\begin{aligned}
g_{\text {KAdS }}:= & -\frac{\Delta-\Delta_{\theta} a^{2} \sin ^{2} \theta}{\Sigma} \mathrm{d} t \otimes \mathrm{d} t+\frac{\Sigma}{\Delta} \mathrm{d} r \otimes \mathrm{d} r+\frac{\Sigma}{\Delta_{\theta}} \mathrm{d} \theta \otimes \mathrm{d} \theta \\
& +\frac{\Delta_{\theta}\left(r^{2}+a^{2}\right)^{2}-\Delta a^{2} \sin ^{2} \theta}{\Xi^{2} \Sigma} \sin ^{2} \theta \mathrm{d} \phi \otimes \mathrm{d} \phi \\
& -\frac{\Delta_{\theta}\left(r^{2}+a^{2}\right)-\Delta}{\Xi \Sigma} a \sin ^{2} \theta(\mathrm{d} t \otimes \mathrm{d} \phi+\mathrm{d} \phi \otimes \mathrm{d} t),
\end{aligned}
$$

where

$$
\Sigma:=r^{2}+a^{2} \cos ^{2} \theta, \quad \Delta_{\theta}:=1-\frac{a^{2}}{l^{2}} \cos ^{2} \theta, \quad \Xi:=1-\frac{a^{2}}{l^{2}}
$$


and $\Delta$ is as in (2.7). We will also write $\Delta_{x}:=1-\frac{a^{2}}{l^{2}} x^{2}$ which arises from the substitution $x=\cos \theta$ in $\Delta_{\theta}$. We also define

$$
\omega_{+}:=\frac{a \Xi}{r_{+}^{2}+a^{2}}, \quad \omega_{-}:=\frac{a \Xi}{r_{-}^{2}+a^{2}}, \quad \omega_{r}:=\frac{a \Xi}{r^{2}+a^{2}} .
$$

Now, we time-orient the patches $\mathcal{R}$ and $\mathcal{B}$ with $-\nabla t_{\mathcal{R}}$ and $-\nabla r_{\mathcal{B}}$, respectively. We also note that $\partial_{t}$ and $\partial_{\phi}$ are Killing fields in each of the patches. The inverse metric reads

$$
\begin{aligned}
g_{\text {KAdS }}^{-1}= & \left(-\frac{\left(r^{2}+a^{2}\right)^{2}}{\Sigma \Delta}+\frac{a^{2} \sin ^{2} \theta}{\Sigma \Delta_{\theta}}\right) \partial_{t} \otimes \partial_{t}+\frac{\Delta}{\Sigma} \partial_{r} \otimes \partial_{r}+\frac{\Delta_{\theta}}{\Sigma} \partial_{\theta} \otimes \partial_{\theta} \\
& +\left(\frac{\Xi^{2}}{\Sigma \Delta_{\theta} \sin ^{2} \theta}-\frac{\Xi^{2} a^{2}}{\Sigma \Delta}\right) \partial_{\phi} \otimes \partial_{\phi} \\
& -\left(\frac{\Xi a\left(r^{2}+a^{2}\right)}{\Delta \Sigma}-\frac{a \Xi}{\Delta_{\theta} \Sigma}\right)\left(\partial_{t} \otimes \partial_{\phi}+\partial_{\phi} \otimes \partial_{t}\right)
\end{aligned}
$$

On $\mathcal{R}$ and $\mathcal{B}$, we define the tortoise coordinate $r^{*}(r)$ by

$$
\frac{\mathrm{d} r^{*}}{\mathrm{~d} r}(r):=\frac{r^{2}+a^{2}}{\Delta(r)}
$$

where $\Delta$ is as in (2.7). For definiteness we set $r^{*}(r=+\infty):=\frac{\pi}{2} l$ on $\mathcal{R}$ and $r^{*}\left(\frac{1}{2}\left(r_{+}+r_{-}\right)\right)=0$ on $\mathcal{B}$.

Eddington-Finkelstein-like coordinates. We also define Eddington-Finkelstein-like coordinates $\left(v, r, \theta, \tilde{\phi}_{+}\right)$in the exterior $\mathcal{R}$ as

$$
v(t, r):=t+r^{*} \chi_{v}(r), \quad \tilde{\phi}_{+}(\phi, r):=\phi+\omega_{+} r^{*}(r) \chi_{v}(r) \bmod 2 \pi,
$$

where $\chi_{v}(r)$ is a smooth monotone cut-off function with $\chi_{v}(r)=1$ for $r \leq$ $r_{+}+\eta$ and $\chi_{v}(r)=0$ for $r \geq r_{+}+2 \eta$ for some $\eta>0$ small enough such that $J^{+}\left(\left\{r>2 r_{+}\right\} \cap\left\{t_{\mathcal{R}}=0\right\}\right) \cap\{v=0\}=\varnothing^{3}$ and $\eta<\frac{r_{+}}{4}$. In these coordinates the spacetime $\left(\mathcal{R}, g_{\mathrm{KAdS}}\right)$ can be extended (see [63] for more details) to a time-oriented Lorentzian manifold ( $\left.\mathcal{D}, g_{\text {KAdS }}\right)$ defined as $\mathcal{D}:=$ $\left\{\left(r, v, \theta, \tilde{\phi}_{+}\right) \in\left(r_{-}, \infty\right) \times \mathbb{R} \times \mathbb{S}^{2}\right\}$. Moreover, the Lorentzian submanifold $\left(\mathcal{D} \cap\left\{r_{-}<r<r_{+}\right\}, g_{\mathrm{KAdS}}\right.$ ) coincides (up to time-orientation preserving isometry) with $\left(\mathcal{B}, g_{\mathrm{KAdS}}\right)$. We identify these regions and denote the (right) event horizon as $\mathcal{H}_{R}:=\left\{r=r_{+}\right\}$. The Killing null generator of the event

\footnotetext{
$\overline{3}$ Note that $\nabla v$ is not timelike everywhere on $\mathcal{R}$, in particular $g(\nabla v, \nabla v)=a^{2} \sin ^{2} \theta \Sigma^{-1} \Delta_{\theta}^{-1}$ for $r \in\left[r_{+}, r_{+}+\eta\right]$.
} 
horizon is

$$
K_{+}:=\partial_{v}+\omega_{+} \partial_{\tilde{\phi}_{+}} \cdot
$$

The Killing field $K_{+}$is called the Hawking vector field and is future-directed and timelike in $\mathcal{R}$, a consequence the Hawking-Reall bound $r_{+}>a l$.

To attach the (left) Cauchy horizon $\mathcal{C H}_{L}$ we introduce in $\mathcal{B}$ further coordinates $\left(v, r, \theta, \tilde{\phi}_{-}\right)$, as

$$
v=t+r^{*}, \quad \tilde{\phi}_{-}(\phi, r):=\phi+\omega_{-} r^{*} \bmod 2 \pi, \quad r=r, \quad \theta=\theta .
$$

In these coordinates, the Lorentzian manifold extends smoothly to $r=r_{-}$and the null hypersurface $\mathcal{C H} \mathcal{H}_{L}:=\left\{r=r_{-}\right\}$is the left Cauchy horizon with null generator

$$
K_{-}:=\partial_{v}+\omega_{-} \partial_{\tilde{\phi}_{-}} \cdot
$$

Note that $\partial_{v}=\partial_{t}$ and $\partial_{\tilde{\phi}_{-}}=\partial_{\phi}$ in $\mathcal{B}$.

To attach the left event horizon $\mathcal{H}_{L}$ we introduce new coordinates on $\mathcal{B}$ defined as $\left(u, r, \theta, \phi_{+}^{*}\right) \in \mathbb{R} \times\left(r_{-}, r_{+}\right) \times \mathbb{S}^{2}$ by

$$
u(t, r):=-t+r^{*}, \quad \phi_{+}^{*}:=\phi-\omega_{+} r^{*} \bmod 2 \pi, r=r, \theta=\theta
$$

on $\mathcal{B}$ and attach $\mathcal{H}_{L}$ as $\mathcal{H}_{L}=\left\{r=r_{+}\right\}$. Similarly, introduce $\left(u, r, \theta, \phi_{-}^{*}\right)$ as

$$
u(t, r):=-t+r^{*}, \quad \phi_{-}^{*}:=\phi-\omega_{-} r^{*} \bmod 2 \pi, r=r, \theta=\theta
$$

on $\mathcal{B}$ and attach the right Cauchy horizon $\mathcal{C H}_{R}$ as $\mathcal{C H}_{R}=\left\{r=r_{-}\right\}$in this coordinate system. Indeed, $K_{+}$and $K_{-}$extend to Killing vector fields expressed as $K_{+}:=-\partial_{u}+\omega_{+} \partial_{\phi_{+}^{*}}$ and $K_{-}:=-\partial_{u}+\omega_{-} \partial_{\phi_{-}^{*}}$. They are past directed Killing generators of $\mathcal{H}_{L}$ and $\mathcal{C H}_{R}$, respectively. Finally, we attach the past and future bifurcation spheres $\mathcal{B}_{\mathcal{H}}$ and $\mathcal{B}_{\mathcal{C H}}$. Formally, they are defined as $\mathcal{B}_{\mathcal{H}}:=\{v=-\infty\} \times\left\{r=r_{+}\right\} \times \mathbb{S}^{2}=\{u=-\infty\} \times\left\{r=r_{+}\right\} \times \mathbb{S}^{2}$ respectively in the coordinates systems $\left(v, r, \theta, \tilde{\phi}_{+}\right)$and $\left(u, r, \theta, \phi_{+}^{*}\right)$. Similarly, we have $\mathcal{B}_{\mathcal{C H}}:=\{v=+\infty\} \times\left\{r=r_{-}\right\} \times \mathbb{S}^{2}=\{u=+\infty\} \times\left\{r=r_{-}\right\} \times \mathbb{S}^{2}$. Finally, we define the Cauchy horizon $\mathcal{C H}:=\mathcal{C H}_{L} \cup \mathcal{C H}_{R} \cup \mathcal{B}_{\mathcal{C H}}$. This is standard and we refer to the preliminary section of [26] for more details. The metric $g_{\text {KAdS }}$ extends to a smooth Lorentzian metric on $\mathcal{B}_{\mathcal{H}}, \mathcal{B}_{\mathcal{C H}}$ and we define $\left(\mathcal{M}_{\mathrm{KAdS}}, g_{\mathrm{KAdS}}\right)$ as the Lorentzian manifold constructed above. Moreover, $T:=\partial_{t}$ and $\Phi:=\partial_{\phi}$ extend to smooth Killing vector fields on $\mathcal{M}_{\text {KAdS }}$ with $K_{+}=T+\omega_{+} \Phi$ and $K_{-}=T+\omega_{-} \Phi$. 
Kerr-AdS-star coordinates. On the exterior region $\mathcal{R}$ we define an additional system of coordinates $\left(t^{*}, r, \theta, \phi^{*}\right)$ from the Boyer-Lindquist coordinates through

$$
t^{*}:=t+A(r), r=r, \theta=\theta, \phi^{*}:=\phi+B(r)-\omega_{+}(t+A(r)),
$$

where $\frac{\mathrm{d} A}{\mathrm{~d} r}=\frac{2 M r}{\Delta\left(1+\frac{r^{2}}{l^{2}}\right)}$ and $\frac{\mathrm{d} B}{\mathrm{~d} r}=\frac{a \Xi}{\Delta}$ and $A=B=0$ at $r=+\infty$. As shown in [66, Sect. 5], these coordinates extend smoothly to the event horizon $\mathcal{H}_{R}$ and we call the coordinates $\left(t^{*}, r, \theta, \phi^{*}\right)$ covering $\mathcal{R} \cup \mathcal{H}_{R}$ Kerr-AdS-star coordinates. Note that the event horizon is characterized as $\mathcal{H}_{R}=\left\{r=r_{+}\right\}$ and that $K_{+}=\partial_{t^{*}}$ in these coordinates.

Foliations and Initial Hypersurface. We foliate the region $\mathcal{R} \cup \mathcal{H}_{R}$ with constant $t^{*}$ hypersurfaces $\Sigma_{t^{*}}$ which are spacelike and intersect the event horizon at $r=r_{+}$. For the initial data we will consider the axisymmetric spacelike hypersurface

$$
\Sigma_{0}:=\Sigma_{t=0}=\mathcal{R} \cap\left\{t_{\mathcal{R}}=0\right\}
$$

Note that $\Sigma_{0}$ does not contain the bifurcation sphere $\mathcal{B}_{\mathcal{H}}$. We will impose initial data on $\Sigma_{0} \cup \mathcal{B}_{\mathcal{H}} \cup \mathcal{H}_{L}$. We will choose the support of our initial data to lie in a compact subset $K \subset \Sigma_{0} \cap\left\{r \geq 2 r_{+}\right\}$. Thus, we assume vanishing data on $\mathcal{H}_{L} \cup \mathcal{B}_{\mathcal{H}}$. This will be made precise in Sect. 6 .

Boundary conditions. Note that the conformal boundary $\mathcal{I}$, expressed formally as $\{r=+\infty\}$, is timelike, as a consequence, ( $\left.\mathcal{M}_{\mathrm{KAdS}}, g_{\mathrm{KAdS}}\right)$ is not globally hyperbolic. Thus, in addition to Cauchy data for (1.2), we will also impose Dirichlet boundary conditions at $\mathcal{I}=\{r=+\infty\}$.

\subsection{Conventions}

If $X$ and $Y$ are two (typically non-negative) quantities, we use $X \lesssim Y$ of $Y \gtrsim X$ to denote that $X \leq C(M, a, l) Y$ for some constant $C(M, a, l)>0$ depending continuously on the black hole parameters $(M, a, l)$, unless explicitly stated otherwise. We also use $X=O(Y)$ for $|X| \lesssim Y$. We use $X \sim Y$ for $X \lesssim Y \lesssim X$ and if the constants appearing in $\lesssim, \gtrsim, \sim$ or $O$ depend on additional parameters $a_{i}$ we include those as subscripts, e.g. $X \lesssim a_{1} a_{2} Y$. Similarly, implicit constants in "sufficiently small" or "sufficiently large" may also depend continuously on $M, a, l$.

In Sect. 6 we will fix parameters $(\mathfrak{m}, \mathfrak{a}) \in \mathscr{P}_{\text {Blow-up }}$ and all constants appearing in $\lesssim$ and $\gtrsim$ throughout Sects. 6, 7, 8 will only depend on this particular choice and on $l>0$ as in (2.6). 
Further, we denote the total variation of a function $f: \mathbb{R} \rightarrow \mathbb{R}$ in the interval $(a, b)$ with $\mathcal{V}_{a, b}(f)$ defined as $\mathcal{V}_{a, b}(f):=\sup _{P} \sum_{i=1}^{n_{p}-1}\left|f\left(x_{i+1}\right)-f\left(x_{i}\right)\right|$, where the supremum runs over the set of all partitions of the given interval, see [94, Chapter 1, §11].

\subsection{Norms and energies}

To state the well-posedness result of (1.2) and the logarithmic decay result on the Kerr-AdS exterior, we define the following norms and energies in the exterior region $\mathcal{R} \cup \mathcal{H}_{R}$. These are based on the works [61,63,65], where more details can be found. In the region $\mathcal{R} \cup \mathcal{H}_{R}$ we let $\$$ and $\not$ be the induced metric and induced connection of the spheres $\mathbb{S}_{t^{*}, r}^{2}$ of constant $t^{*}$ and $r$. For a smooth function $\psi$ we denote $|\not \nabla \ldots \not \nabla \psi|^{2}=$ $\phi^{A A^{\prime}} \ldots g^{B B^{\prime}} \not_{A} \ldots \not_{B} \bar{\psi} \nabla_{A^{\prime}} \ldots \not_{B^{\prime}} \psi$. Now, we define energy densities in Kerr-AdS-star coordinates as

$$
\begin{aligned}
e_{1}[\psi]:= & \frac{1}{r^{2}}\left|\partial_{t^{*}} \psi\right|^{2}+r^{2}\left|\partial_{r} \psi\right|^{2}+|\not \nabla \psi|^{2}+|\psi|^{2}, \\
e_{2}[\psi]:= & e_{1}[\psi]+e_{1}\left[\partial_{t^{*}} \psi\right]+\sum_{i=1}^{3} e_{1}\left[\Omega_{i} \psi\right]+r^{4}\left|\partial_{r} \partial_{r} \psi\right|^{2} \\
& +r^{2}\left|\partial_{r} \not \psi\right|^{2}+|\not \varnothing \not \psi|^{2},
\end{aligned}
$$

and analogously for higher order energy densities. Here, $\left(\Omega_{i}\right)_{i=1,2,3}$ denote the angular momentum operators on the unit sphere in $\theta, \phi^{*}$ coordinates. We also define the energy norms on constant $t^{*}$ hypersurfaces as

$$
\begin{aligned}
\|\psi\|_{H_{\mathrm{AdS}}^{0, s}\left(\Sigma_{t^{*}}\right)}^{2} & =\int_{\Sigma_{t^{*}}} r^{s}|\psi|^{2} r^{2} \mathrm{~d} r \sin \theta \mathrm{d} \theta \mathrm{d} \phi^{*} \\
\|\psi\|_{H_{\mathrm{AdS}}^{1, s}\left(\Sigma_{t^{*}}\right)}^{2} & =\int_{\Sigma_{t^{*}}} r^{s}\left(r^{2}\left|\partial_{r} \psi\right|^{2}+|\not \nabla \psi|^{2}+|\psi|^{2}\right) r^{2} \mathrm{~d} r \sin \theta \mathrm{d} \theta \mathrm{d} \phi^{*} \\
\|\psi\|_{H_{\mathrm{AdS}}^{2, s}\left(\Sigma_{t^{*}}\right)}^{2} & =\|\psi\|_{H_{\mathrm{AdS}}^{1, s}\left(\Sigma_{t^{*}}\right)}^{2}+\int_{\Sigma_{t^{*}}} r^{s}\left(r^{4}\left|\partial_{r} \partial_{r} \psi\right|^{2}+r^{2}\left|\not \nabla \partial_{r} \psi\right|^{2}\right. \\
& \left.+|\not \nabla \not \nabla|^{2}\right) r^{2} \mathrm{~d} r \sin \theta \mathrm{d} \theta \mathrm{d} \phi^{*}
\end{aligned}
$$

We now denote the space $H_{\text {AdS }}^{k, s}\left(\Sigma_{t^{*}}\right)$ as the space of functions with $\nabla^{i} \psi \in$ $L_{l o c}^{2}\left(\Sigma_{t^{*}}\right)$ for $i=0, \ldots, k$ and such that $\|\psi\|_{H_{\mathrm{AdS}}^{k, s}\left(\Sigma_{t^{*}}\right)}^{2}<\infty$ and we denote with $C H_{\text {AdS }}^{k}$ the space of functions $\psi$ on $\mathcal{R} \cup \mathcal{H}_{R}$ such that $\psi \in$ 
$\bigcap_{q=0, \ldots, k} C^{q}\left(\mathbb{R}_{t^{*}} ; H_{\mathrm{AdS}}^{k-q, s_{q}}\left(\Sigma_{t^{*}}\right)\right)$, where $s_{k}=-2, s_{k-1}=0$ and $s_{j}=0$ for $j=0, \ldots, k-2$.

\subsection{Well-posedness and log-decay on the exterior region}

In the following we state well-posedness for (1.2) and decay solutions with Dirichlet boundary conditions. The following theorem is a summary of results by Holzegel, Smulevici and Warnick shown in $[60,61,63,65,66]$.

Theorem $2([60,61,63,65,66])$ Let the initial data $\Psi_{0}, \Psi_{1} \in C_{c}^{\infty}\left(\Sigma_{0}\right)$. Assume Dirichlet boundary conditions at $\mathcal{I}$ and vanishing incoming data on $\mathcal{H}_{L} \cup \mathcal{B}_{\mathcal{H}}$. Then, there exists a unique solution $\psi \in C^{\infty}\left(\mathcal{M}_{\mathrm{KAdS}} \backslash \mathcal{C H}\right)$ of $(1.2)$ such that $\left.\psi\right|_{\Sigma_{0}}=\Psi_{0},\left.n_{\Sigma_{0}} \psi\right|_{\Sigma_{0}}=\Psi_{1}, \psi \mid \mathcal{H}_{L} \cup \mathcal{B}_{\mathcal{H}}=0$. The solution satisfies $\psi\left\lceil i_{\mathcal{R} \cup \mathcal{H}_{R}} \in C H_{\text {AdS }}^{k}\right.$ for every $k \in \mathbb{N}$. We also have boundedness of the energy

$$
\int_{\Sigma_{t_{2}^{*}}} e_{1}[\psi] r^{2} \sin \theta \mathrm{d} r \mathrm{~d} \theta \mathrm{d} \phi^{*} \lesssim \int_{\Sigma_{t_{1}^{*}}} e_{1}[\psi] r^{2} \sin \theta \mathrm{d} r \mathrm{~d} \theta \mathrm{d} \phi^{*}
$$

for $t_{2}^{*} \geq t_{1}^{*} \geq 0$ as well as for all higher order energies. Further, the energy along the event horizon is bounded by the initial energy as

$$
\begin{array}{r}
\sum_{\substack{1 \leq i_{1}+i_{2} \leq k \\
i_{2} \geq 1}} \int_{\mathcal{H}_{R} \cap\left\{t^{*} \geq t_{0}^{*}\right\}}\left|\nabla^{i_{1}} K_{+}^{i_{2}} \psi\right|^{2} r^{2} \sin \theta \mathrm{d} v \mathrm{~d} \theta \mathrm{d} \tilde{\phi}_{+} \\
\lesssim k \int_{\Sigma_{t_{0}^{*}}} e_{k}[\psi] r^{2} \sin \theta \mathrm{d} r \mathrm{~d} \theta \mathrm{d} \phi .
\end{array}
$$

for any $t_{0}^{*} \geq 0$.

Moreover, the energy of $\psi$ decays

$$
\int_{\Sigma_{t^{*}}} e_{1}[\psi] r^{2} \sin \theta \mathrm{d} r \mathrm{~d} \theta \mathrm{d} \phi^{*} \lesssim \frac{1}{\left[\log \left(2+t^{*}\right)\right]^{2}} \int_{\Sigma_{t_{0}^{*}}} e_{2}[\psi] r^{2} \sin \theta \mathrm{d} r \mathrm{~d} \theta \mathrm{d} \phi
$$

for all $t^{*} \geq t_{0}^{*} \geq 0$ and similar estimates hold for all higher order energies. Similarly, by commuting and applications of the Sobolev embeddings, $\psi$ and all its derivatives also decay pointwise

$$
\sum_{0 \leq i_{1}+i_{2}+i_{3} \leq k}\left|\not^{i_{1}} \partial_{t^{*}}^{i_{2}} \partial_{r}^{i_{3}} \psi\right|^{2} \lesssim_{k} \frac{1}{\left[\log \left(2+t^{*}\right)\right]^{2}} \int_{\Sigma_{t_{0}^{*}}} e_{k+3}[\psi] r^{2} \sin \theta \mathrm{d} r \mathrm{~d} \theta \mathrm{d} \phi
$$


for $t^{*} \geq t_{0}^{*} \geq 0$.

By general theory (a local in time energy estimate) all norms on the righthand side of (2.32)-(2.35) are bounded in terms of a non-degenerate energy of $\psi$ on $\Sigma_{0}$, i.e. in terms of weighted Sobolev norms (of appropriate order) of $\Psi_{0}$ and $\Psi_{1}$ on $\Sigma_{0}$. In particular, since $\Psi_{0}, \Psi_{1}$ are smooth and compactly supported, all right-hand sides of (2.32)-(2.35) are finite.

It should be noted that (2.33) merely gives a bound on $\int_{\mathcal{H}_{R}}\left|K_{+} \psi\right|^{2}$ which does not control the full $L^{2}$-norm of $\psi$ along in the event horizon. However, one obtains control of the $L^{2}$-norm via an "inverse-commutation" argument relying on [119, Theorem 4.9].

Proposition 2.1 Let the initial data $\Psi_{0}, \Psi_{1} \in C_{c}^{\infty}\left(\Sigma_{0}\right)$. Assume Dirichlet boundary conditions at $\mathcal{I}$ and vanishing incoming data on $\mathcal{H}_{L} \cup \mathcal{B}_{\mathcal{H}}$. In view of Theorem 2 , denote by $\psi$ the unique solution with $\left.\psi\right|_{\Sigma_{0}}=\Psi_{0},\left.n_{\Sigma_{0}} \psi\right|_{\Sigma_{0}}=\Psi_{1}$, $\psi\left\lceil\mathcal{H}_{L} \cup \mathcal{B}_{\mathcal{H}}=0\right.$. Then,

$$
D_{\mathcal{H}_{R}}^{k}[\psi]:=\sum_{0 \leq i_{1}+i_{2} \leq k} \int_{\mathcal{H}_{R}}\left|\nabla^{i_{1}} K_{+}^{i_{2}} \psi\right|^{2} r^{2} \sin \theta \mathrm{d} v \mathrm{~d} \theta \mathrm{d} \tilde{\phi}_{+}<\infty
$$

for each $k \in \mathbb{N}$.

Proof By a local in time energy estimate it suffices to show that

$$
\sum_{0 \leq i_{1}+i_{2} \leq k} \int_{\mathcal{H}_{R} \cap\left\{t^{*} \geq 0\right\}}\left|\nabla^{i_{1}} K_{+}^{i_{2}} \psi\right|^{2} r^{2} \sin \theta \mathrm{d} v \mathrm{~d} \theta \mathrm{d} \tilde{\phi}_{+}<\infty .
$$

Further, we also have that the solution $\psi$ has finite energy on $\Sigma_{t_{0}^{*}}$ for $t_{0}^{*}:=0$ of all orders in the sense that

$$
\int_{\Sigma_{t_{0}^{*}}^{*}} e_{k}[\psi] r^{2} \sin \theta \mathrm{d} r \mathrm{~d} \theta \mathrm{d} \phi<\infty
$$

for every $k \in \mathbb{N}$. We denote $\left(\psi_{0}, \psi_{1}\right):=\left(\psi, K_{+} \psi\right)\left\lceil\Sigma_{t_{0}^{*}}\right.$.

In view of the above and (2.33) in Theorem 2, to obtain (2.36), it suffices to show that there exist data $\left(\tilde{\psi}, K_{+} \tilde{\psi}\right)\left\lceil\Sigma_{t_{0}^{*}}=\left(\tilde{\psi}_{0}, \tilde{\psi}_{1}\right)\right.$ for $(1.2)$ such that the arising solution $\tilde{\psi}$ satisfies $K_{+} \tilde{\psi}=\psi$ and moreover $\left(\tilde{\psi}_{0}, \tilde{\psi}_{1}\right)$ are sufficiently regular as to apply the (twisted) energy estimate associated to $K_{+}$. We set $\tilde{\psi}_{1}:=\psi_{0}$ and it remains to construct $\tilde{\psi}_{0}$ via inverting an elliptic operator to ensure $K_{+} \tilde{\psi}=\psi$.

To construct $\tilde{\psi}_{0}$ we will use [119, Theorem 4.9]. To apply it we briefly recall the theory developed in $[66,119]$. Note from [119, Lemma 5.2] and the Hawking-Recall bound (2.8) that the Kerr-AdS exterior to the future of $\Sigma_{t_{0}^{*}}$ 
is a globally stationary asymptotically Anti-de Sitter black hole spacetime in the sense of [119, Definition 2.6] with stationary vector field $K_{+}$. Thus, we can apply the general framework of [119]. Following [119] we write (1.2) as $L \psi=0$ for a strongly hyperbolic operator with $W=0, V=\frac{2}{l^{2}}$ as in [119, Definition 2.7], more precisely, $L:=A\left(\square_{g}+\frac{2}{l^{2}}\right)$ for $A=\frac{-1}{g\left(\nabla t^{*}, \nabla t^{*}\right)}$. As in $\left[119\right.$, p. 998] we decompose $L \tilde{\psi}=P_{2} \tilde{\psi}+P_{1} K_{+} \tilde{\psi}+K_{+} K_{+} \tilde{\psi}$, where $P_{1}$ is a differential operator of first order on $\Sigma_{t_{0}^{*}}$ and $P_{2}$ is a (degenerate) elliptic operator on $\Sigma_{t_{0}^{*}}$. We further recall the following natural norms from [119, p. 976]:

$$
\begin{aligned}
\|\tilde{\psi}\|_{\underline{L}^{2}\left(\Sigma_{t_{0}^{*}}\right)}^{2} & :=\int_{\Sigma_{t_{0}^{*}}}|\tilde{\psi}|^{2} r^{-1} \operatorname{dvol}_{\Sigma_{t_{0}^{*}}^{*}},\|\tilde{\psi}\|_{\underline{H}^{1}\left(\Sigma_{t_{0}^{*}}\right)}^{2} \\
& :=\int_{\Sigma_{t_{0}^{*}}}\left(|\tilde{\nabla} \tilde{\psi}|^{2}+|\tilde{\psi}|^{2} r^{-2}\right) r \operatorname{dvol}_{\Sigma_{t_{0}^{*}}},
\end{aligned}
$$

where $\tilde{\nabla}_{\mu} \tilde{\psi}:=r^{-1} \nabla_{\mu}(r \tilde{\psi}), \tilde{\nabla}_{\mu}^{\dagger} \tilde{\psi}:=-r \nabla_{\mu}\left(r^{-1} \tilde{\psi}\right)$ are the twisted derivatives and the norms in (2.39) are with respect to the induced metric on $\Sigma_{t_{0}^{*}}$. As in [119] we define respectively $\underline{L}^{2}\left(\Sigma_{t_{0}^{*}}\right)$ and $\underline{H}^{1}\left(\Sigma_{t_{0}^{*}}\right)$ as the completion of smooth functions on $\Sigma_{t_{0}^{*}}$ which are supported away from $\mathcal{I}$ in the norms $\|\cdot\|_{\underline{L}^{2}\left(\Sigma_{t_{0}^{*}}\right)}$ and $\|\cdot\|_{\underline{H}^{1}\left(\Sigma_{t_{0}^{*}}\right)}$, respectively.

In order to construct $\tilde{\psi}_{0}$ we need to invert $P_{2}$, more precisely, we need to solve $P_{2} \tilde{\psi}_{0}=-P_{1} \psi_{0}-\psi_{1}$, where we note that $P_{1}: \underline{H}^{1}\left(\Sigma_{t_{0}^{*}}\right) \rightarrow$ $\underline{L}^{2}\left(\Sigma_{t_{0}^{*}}\right)$ is a bounded operator (see [119, p. 1002]). In particular, we have $P_{1} \psi_{0}+\psi_{1} \in \underline{L}^{2}\left(\Sigma_{t_{0}^{*}}\right)$ in view of (2.38). We note that $P_{2}=\hat{L}_{0}$, where $\hat{L}_{0}=\hat{L}_{s=0}: \operatorname{dom}\left(\hat{L}_{s=0}\right) \rightarrow \underline{L}^{2}\left(\Sigma_{t_{0}^{*}}\right)$ is as in [119, eqn. (4.1), $\left.s=0\right]$. Now, we apply [119, Theorem 4.9] with $k=0$ and $s=0$. Indeed, $k=0$ and $s=0$ are valid because $w_{L}=0$ (recall $W=0$ and [119, Definition 3.7]). Since $0 \notin \Lambda_{\mathrm{QNF}}^{0}$ (no stationary solutions exist [119, Corollary 1.3]), we have from [119, Theorem 4.9] that the operator $P_{2}^{-1}: \underline{L}^{2}\left(\Sigma_{t_{0}^{*}}\right) \rightarrow \underline{H}^{1}\left(\Sigma_{t_{0}^{*}}\right)$ exists and is bounded. Hence, $\tilde{\psi}_{0}=-P_{2}^{-1}\left(P_{1} \psi_{0}+\psi_{1}\right) \in \underline{H}^{1}\left(\Sigma_{t_{0}^{*}}\right)$. (In fact, $\tilde{\psi}_{0}$ can be shown to be more regular which is however not needed for the proof.)

Finally, let $\tilde{\psi}$ be the unique solution to (1.2) arising from initial data $\left.\left(\tilde{\psi}, K_{+} \tilde{\psi}\right)\right|_{\Sigma_{t_{0}^{*}}}=\left(\tilde{\psi}_{0}, \tilde{\psi}_{1}\right)$. This is well-posed by [119, Theorem 2.3]. Then, by construction we have that $K_{+} \tilde{\psi}=\psi$. Now, by the twisted energy estimate for $\tilde{\psi}$ associated to $K_{+}$(see e.g. [66, Proposition 3] and [119, Theorem 3.4 (i), $\gamma=0]$ ) we have $\int_{\mathcal{H}_{R} \cap\left\{t^{*} \geq t_{0}^{*}\right\}}|\psi|^{2} r^{2} \mathrm{~d} v \sin \theta \mathrm{d} \theta \mathrm{d} \tilde{\phi}_{+} \lesssim$ $\int_{t^{*} \geq t_{0}^{*}}\left|K_{+} \tilde{\psi}\right|^{2} r_{+}^{2} \mathrm{~d} t^{*} \sin \theta \mathrm{d} \theta \mathrm{d} \phi^{*} \lesssim\left\|\tilde{\psi}_{0}\right\|_{\underline{H}^{1}\left(\Sigma_{t_{0}^{*}}\right)}+\left\|\tilde{\psi}_{1}\right\|_{\underline{L}^{2}\left(\Sigma_{t_{0}^{*}}\right)}<\infty$. This concludes the proof. 


\subsection{Separation of variables: radial o.d.e., angular o.d.e. and coupling constants $\lambda_{m \ell}(a \omega)$}

The wave equation (1.2) is formally separable [13] and we can consider pure mode solutions in the Boyer-Lindquist coordinates of the form

$$
\psi(t, r, \theta, \phi)=\frac{u(r)}{\sqrt{r^{2}+a^{2}}} e^{-i \omega t} S_{m \ell}(a \omega, \cos \theta) e^{i m \phi}, m \in \mathbb{Z}, \omega \in \mathbb{R}
$$

for two unknown functions $u(r)$ and $S_{m \ell}(a \omega, \cos \theta)$. Plugging this ansatz into (1.2) leads to a coupled system of o.d.e's. The angular o.d.e. is the eigenvalue equation of the operator $P(a \omega)$ which reads

$$
P(a \omega) S_{m \ell}(a \omega, \cos \theta)=\lambda_{m \ell}(a \omega) S_{m \ell}(a \omega, \cos \theta),
$$

where

$$
\begin{aligned}
P(\xi) f= & P_{m}(\xi) f=-\frac{1}{\sin \theta} \partial_{\theta}\left(\Delta_{\theta} \sin \theta \partial_{\theta} f\right) \\
& +\frac{\Xi^{2} m^{2}}{\Delta_{\theta} \sin ^{2} \theta} f-\Xi \xi^{2} \Delta_{\theta}^{-1} \cos ^{2} \theta f \\
& -2 m \xi \frac{\Xi}{\Delta_{\theta}} \frac{a^{2}}{l^{2}} \cos ^{2} \theta f+\frac{2}{l^{2}} a^{2} \sin ^{2} \theta f, \quad \xi \in \mathbb{R} .
\end{aligned}
$$

The operator (2.42) is realized as a self-adjoint operator on a suitable domain in $L^{2}((0, \pi) ; \sin \theta \mathrm{d} \theta)$. As a Sturm-Liouville operator, the spectrum of $P(a \omega)$ consists of simple eigenvalues $\lambda_{m \ell}(a \omega)$, where $\ell \in \mathbb{Z}_{\geq|m|}$ labels the eigenvalue in ascending order. The eigenvalues $\lambda_{m \ell}(a \omega)$ of $P(a \omega)$ couple the angular o.d.e. to the radial o.d.e.

$$
-u^{\prime \prime}+\left(V-\omega^{2}\right) u=0,
$$

where $^{\prime}:=\frac{\mathrm{d}}{\mathrm{d} r^{*}}$. We also use the notation $\tilde{V}:=V-\omega^{2}$, where the potential $V$ is given by

$$
V=V_{0}+V_{1}
$$

with purely radial part

$$
V_{1}:=\frac{-\Delta^{2} 3 r^{2}}{\left(r^{2}+a^{2}\right)^{4}}+\Delta \frac{5 \frac{r^{4}}{l^{2}}+3 r^{2}\left(1+\frac{a^{2}}{l^{2}}\right)-4 M r+a^{2}}{\left(r^{2}+a^{2}\right)^{3}}-\frac{2 \Delta}{l^{2}} \frac{1}{r^{2}+a^{2}}
$$


and frequency dependent part

$$
V_{0}:=\frac{\Delta\left(\lambda_{m \ell}(a \omega)+\omega^{2} a^{2}\right)-\Xi^{2} a^{2} m^{2}-2 m \omega a \Xi\left(\Delta-\left(r^{2}+a^{2}\right)\right)}{\left(r^{2}+a^{2}\right)^{2}} .
$$

We will be particularly interested in the case for which the frequency $\omega$ coincides with the interior scattering poles, i.e. $\omega=\omega_{-} m$. Moreover, in order to be in the regime of stable trapping on the exterior we also want $|\omega|$ and $|m|$ to be large. Hence, we will think of $\frac{1}{m}$ as a small semiclassical parameter. In particular, setting $\omega=\omega_{-} m$ in (2.43) and separating out the $m^{2}$ we obtain

$$
-u^{\prime \prime}+\left(m^{2} V_{\text {main }}+V_{1}\right) u=0,
$$

where $V_{1}$ is as in (2.45) and

$$
\begin{aligned}
V_{\text {main }} & :=\frac{V_{0}\left(\omega=\omega_{-} m\right)-\omega_{-}^{2} m^{2}}{m^{2}} \\
& =\frac{\Delta}{\left(r^{2}+a^{2}\right)^{2}}\left(\frac{\lambda_{m \ell}\left(a \omega_{-} m\right)}{m^{2}}+\omega_{-}^{2} a^{2}-2 a \omega_{-} \Xi\right)-\left(\omega_{-}-\omega_{r}\right)^{2} .
\end{aligned}
$$

In (2.48) we also used $\omega_{r}=\frac{a \Xi}{r^{2}+a^{2}}$ as defined in (2.16). We begin our analysis with the angular o.d.e. (2.41) in the following Sect. 3.

\section{The angular o.d.e.}

For the operator $P(\xi)$ as in (2.42) we change variables to $x=\cos \theta$. This is a unitary transformation and thus, the eigenvalues of $P(\xi)$ are equal to the eigenvalues of $P_{x}$ given by

$$
\begin{aligned}
P_{x}(\xi):= & -\frac{\mathrm{d}}{\mathrm{d} x}\left(\Delta_{x}\left(1-x^{2}\right) \frac{\mathrm{d}}{\mathrm{d} x} \cdot\right)+\frac{\Xi^{2} m^{2}}{\Delta_{x}\left(1-x^{2}\right)} \\
& -\Xi \xi^{2} \frac{x^{2}}{\Delta_{x}}-2 m \xi \frac{\Xi}{\Delta_{x}} \frac{a^{2}}{l^{2}} x^{2}+\frac{2}{l^{2}} a^{2}\left(1-x^{2}\right) .
\end{aligned}
$$

The Sturm-Liouville operator $P_{x}$ is realized as a self-adjoint operator acting on a domain $\mathcal{D} \subset L^{2}(-1,1)$ which can be explicitly characterized as

$$
\begin{aligned}
\mathcal{D}=\left\{f \in L^{2}(-1,1): f \in A C^{1}(-1,1), P_{x} f \in L^{2}(-1,1),\right. & \\
& \left.\lim _{x \rightarrow \pm 1}\left(1-x^{2}\right) f^{\prime}(x)=0 \text { if } m=0\right\},
\end{aligned}
$$


see e.g. [110, Chapter 10.3, Theorem 10.7] which, mutatis mutandis, also applies to (3.1) and (3.2). In (3.2), $A C^{1}(-1,1)$ denotes the space of differentiable functions with absolutely continuous derivative.

Having the same spectrum as $P$, the operator $P_{x}$ has eigenvalues $\left(\lambda_{m \ell}(\xi)\right)_{\ell \geq|m|}$ with corresponding real-analytic eigenfunctions $S_{m \ell}=S_{m \ell}(\xi, x)$ which satisfy

$$
P_{x} S_{m \ell}=\lambda_{m \ell} S_{m \ell} \quad \text { and } \quad\left\|S_{m \ell}(\xi)\right\|_{L^{2}(-1,1)}=1 .
$$

We note that for $\xi=a=0$, the eigenvalues $\left(\lambda_{m \ell}\right)_{\ell \geq|m|}$ reduce to the eigenvalues of the Laplacian on the sphere $\lambda_{m \ell}(a=\xi=0)=\ell(\ell+1)$. We also define the shifted eigenvalues

$$
\Lambda_{m \ell}(\xi):=\lambda_{m \ell}(\xi)+\xi^{2}
$$

A computation (see [63, Proof of Lemma 5.1]) shows that

$$
P_{x}(\xi)+\xi^{2}-\frac{2}{l^{2}} a^{2}\left(1-x^{2}\right) \geq \Xi^{2} P_{x}(\xi=0, a=0)
$$

in the sense of self-adjoint operators acting on $\mathcal{D} \subset L^{2}(-1,1)$. Hence,

$$
\Lambda_{m \ell}(\xi) \geq \Xi^{2} \ell(\ell+1) \geq \Xi^{2}|m|(|m|+1) .
$$

Having recalled basic properties of the angular problem we now focus on the interior scattering poles $\omega=\omega_{-} m$ for large $m$. In particular, we will only consider $m \neq 0$ for the rest of Sect. 3 .

\subsection{Angular potential $W_{1}$ at interior scattering poles in semi-classical limit}

In the current Sect. 3.1 and in the following Sect. 3.2 we will consider the operator

$$
\begin{aligned}
P_{\omega_{-}}:= & P_{x}\left(\xi=a m \omega_{-}\right) \\
= & -\frac{\mathrm{d}}{\mathrm{d} x}\left(\Delta_{x}\left(1-x^{2}\right) \frac{\mathrm{d}}{\mathrm{d} x} \cdot\right)+\frac{\Xi^{2} m^{2}}{\Delta_{x}\left(1-x^{2}\right)} \\
& -\Xi a^{2} m^{2} \omega_{-}^{2} \frac{x^{2}}{\Delta_{x}}-2 m^{2} a \omega_{-} \frac{\Xi}{\Delta_{x}} \frac{a^{2}}{l^{2}} x^{2}+\frac{2}{l^{2}} a^{2}\left(1-x^{2}\right)
\end{aligned}
$$


with corresponding eigenvalues $\lambda_{m \ell}:=\lambda_{m \ell}\left(a \omega_{-} m\right)$. We re-write the eigenvalue problem

$$
P_{\omega_{-}} f=\lambda f
$$

as

$$
\tilde{P}_{\omega_{-}} f=0,
$$

where

$$
\begin{aligned}
\tilde{P}_{\omega_{-}} & :=-\Delta_{x}\left(1-x^{2}\right) \frac{\mathrm{d}}{\mathrm{d} x}\left(\Delta_{x}\left(1-x^{2}\right) \frac{\mathrm{d}}{\mathrm{d} x} \cdot\right)+m^{2} W_{1}(x)+P_{\text {error }}, \\
P_{\text {error }} & :=\Delta_{x}\left(1-x^{2}\right) \frac{2}{l^{2}} a^{2}\left(1-x^{2}\right)
\end{aligned}
$$

and

$$
W_{1}:=\Xi^{2}-\left[\Xi a^{2} \omega_{-}^{2}+2 a \omega_{-} \Xi \frac{a^{2}}{l^{2}}\right] x^{2}\left(1-x^{2}\right)-\tilde{\lambda} \Delta_{x}\left(1-x^{2}\right),
$$

with

$$
\tilde{\lambda}:=\frac{\lambda}{m^{2}}
$$

In the semi-classical limit $m^{2} \rightarrow \infty$ we consider $P_{\text {error }}$ as a perturbation and $W_{1}$ determines the leading order terms of the eigenvalues and eigenfunctions. Consequently, our analysis focuses on $W_{1}$ which we analyze in the following lemma.

Lemma 3.1 Let $W_{1}$ be the angular potential defined in (3.12).

1. For $\tilde{\lambda}<\Xi^{2}$, we have $W_{1}>0$ for $x \in[0,1]$.

2. For $\tilde{\lambda}=\Xi^{2}$, we have $W_{1}>0$ on $(0,1]$ and $W_{1}(x=0)=0$.

3. For $\tilde{\lambda}>\Xi^{2}$, the potential $W_{1}$ has exactly one root in $x \in[0,1]$ and satisfies

$$
\frac{\mathrm{d} W_{1}}{\mathrm{~d} x} \gtrsim \tilde{\lambda} x
$$

for $x \in[0,1]$. We call this root $x_{0}$ which also satisfies $x_{0} \in(0,1)$.

Proof We start by expanding $W_{1}$ and obtain

$$
W_{1}(x)=\Xi^{2}-\tilde{\lambda}+a_{1} x^{2}+a_{2} x^{4}
$$


with

$$
\begin{aligned}
a_{1} & =\tilde{\lambda}\left(1+\frac{a^{2}}{l^{2}}\right)-\frac{a^{4}\left(a^{2}-l^{2}\right)^{2}\left(a^{2}+l^{2}+2 r_{-}^{2}\right)}{l^{6}\left(a^{2}+r_{-}^{2}\right)^{2}} \\
& =\Xi^{2}\left(1+\frac{a^{2}}{l^{2}}\right)-\frac{a^{4}\left(a^{2}-l^{2}\right)^{2}\left(a^{2}+l^{2}+2 r_{-}^{2}\right)}{l^{6}\left(a^{2}+r_{-}^{2}\right)^{2}}+\left(\tilde{\lambda}-\Xi^{2}\right)\left(1+\frac{a^{2}}{l^{2}}\right) \\
& =\frac{(a-l)^{2}(a+l)^{2}\left(2 a^{2} l^{2} r_{-}^{2}+\left(a^{2}+l^{2}\right) r_{-}^{4}\right)}{l^{6}\left(a^{2}+r_{-}^{2}\right)^{2}}+\left(\tilde{\lambda}-\Xi^{2}\right)\left(1+\frac{a^{2}}{l^{2}}\right)
\end{aligned}
$$

and

$$
a_{2}=\frac{a^{4}\left(a^{2}-l^{2}\right)^{2}\left(a^{2}+l^{2}+2 r_{-}^{2}\right)}{l^{6}\left(a^{2}+r_{-}^{2}\right)^{2}}-\frac{a^{2}}{l^{2}} \tilde{\lambda}
$$

We also note that

$$
W_{1}(x=0)=\Xi^{2}-\tilde{\lambda} .
$$

We now consider the case $\tilde{\lambda} \geq \Xi^{2}$ and remark that

$$
\frac{\mathrm{d} W_{1}}{\mathrm{~d} x}=2 a_{1} x+4 a_{2} x^{3} .
$$

We look at two cases now, $a_{2} \geq 0$ and $a_{2}<0$. If $a_{2} \geq 0$, then we directly infer that $\frac{\mathrm{d} W_{1}}{\mathrm{~d} x} \geq 2 a_{1} x$. If $a_{2}<0$, then we use that $x^{3}<x$ and estimate

$$
\frac{\mathrm{d} W_{1}}{\mathrm{~d} x}=2 a_{1} x+4 a_{2} x^{3} \geq\left(2 a_{1}+4 a_{2}\right) x .
$$

Now, a direct computation yields

$$
2 a_{1}+4 a_{2}=2 \Xi\left(\Xi \frac{a^{2}}{l^{2}} \frac{a^{2}+l^{2}+2 r_{-}^{2}}{\left(a^{2}+r_{-}^{2}\right)^{2}}+\tilde{\lambda}\right) \gtrsim \tilde{\lambda} .
$$

Note that this shows (3.14) for $x \in[0,1]$ and we conclude 3 . Together with (3.18), this also shows that $W_{1}(x)>0$ for $x \in(0,1]$ and $\tilde{\lambda}=\Xi^{2}$ such that we have 2 .

Finally for $\tilde{\lambda}<\Xi^{2}$, we have $W_{1}>0$ everywhere because for each fixed $x$, the function $\tilde{\lambda} \mapsto W_{1}(x)$ is strictly decreasing. 


\subsection{Angular eigenvalues at interior scattering poles with}

$$
\lambda_{m_{i} \ell_{i}}=\tilde{\lambda}_{0} m_{i}^{2}+O(1)
$$

For the proof of the Baire-genericity of the set $\mathscr{P}_{\text {Blow-up in Sect. } 5.2 \text { we will }}$ use that there exists a sequence of angular eigenvalues of the form $\lambda_{m_{i} \ell_{i}}=$ $\lambda_{m_{i} \ell_{i}}\left(\omega=\omega_{-} m\right)=\tilde{\lambda}_{0} m_{i}^{2}+O(1)$ at the interior scattering poles. To show this, we will use the following well-known result (Proposition 3.1) on the semi-classical distribution of eigenvalues. The proof of Proposition 3.1 relies on suitable connection formulas of Airy functions and can be found in [94, Chapter 13, §8-§9.1]. We also recall the definition $\mathcal{V}$ of the total variation as in Sect. 2.3.

Proposition 3.1 ([94, Chapter 13, §8.2-\$9.1]) Consider a parameter $\epsilon \epsilon$ $I_{0}:=\left[\epsilon_{0}, \epsilon_{1}\right]$. Let $f_{\epsilon}(x), g_{\epsilon}(x) \in C^{2}\left(\mathbb{R}_{x}\right)$ for all $\epsilon \in I_{0}$ where we assume that $f_{\epsilon}$ and $g_{\epsilon}$ depend continuously on $\epsilon$. Assume that $f_{\epsilon}(x) /\left[\left(x-\hat{x}_{0}(\epsilon)\right)(x-\right.$ $\left.\left.x_{0}(\epsilon)\right)\right]$ is positive and bounded away from zero uniformly for $\epsilon \in I_{0}$. In particular, assume that $f_{\epsilon}$ has two simple roots at $\hat{x}_{0}(\epsilon)<x_{0}(\epsilon)$ with $-\infty<\inf _{\epsilon} \hat{x}_{0}(\epsilon)$ and $\sup _{\epsilon} x_{0}(\epsilon)<+\infty$. Assume further that the roots do not coalesce, i.e. that there exists a $x_{1} \in \mathbb{R}$ with $\sup _{\epsilon} \hat{x}_{0}(\epsilon)<x_{1}<\inf _{\epsilon} x_{0}(\epsilon)$. Assume that for all $\epsilon \in I_{0}$ and for some $c>0$ sufficiently large, $\int_{c}^{x} \sqrt{f_{\epsilon}}$ diverges as $x \rightarrow+\infty$ and $\int_{x}^{-c} \sqrt{f_{\epsilon}}$ diverges as $x \rightarrow-\infty$.

To make the above statements quantitative, define the error-control functions

$$
\begin{aligned}
& H_{\epsilon}(x):=\int_{x_{0}(\epsilon)}^{x} \frac{1}{\left|f_{\epsilon}\right|^{\frac{1}{4}}} \frac{\mathrm{d}^{2}}{\mathrm{~d} y^{2}}\left(\frac{1}{\left|f_{\epsilon}\right|^{\frac{1}{4}}}\right)-\frac{g_{\epsilon}}{\left|f_{\epsilon}\right|^{\frac{1}{2}}}-\frac{5\left|f_{\epsilon}\right|^{2}}{16\left|\zeta_{\epsilon}\right|^{3}} \mathrm{~d} y, \\
& \hat{H}_{\epsilon}(x):=\int_{\hat{x}_{0}(\epsilon)}^{x} \frac{1}{\left|f_{\epsilon}\right|^{\frac{1}{4}}} \frac{\mathrm{d}^{2}}{\mathrm{~d} y^{2}}\left(\frac{1}{\left|f_{\epsilon}\right|^{\frac{1}{4}}}\right)-\frac{g_{\epsilon}}{\left|f_{\epsilon}\right|^{\frac{1}{2}}}-\frac{5\left|f_{\epsilon}\right|^{2}}{16\left|\hat{\zeta}_{\epsilon}\right|^{3}} \mathrm{~d} y
\end{aligned}
$$

for $\left|\zeta_{\epsilon}\right|^{3}:=\left|\frac{3}{2} \int_{x_{0}(\epsilon)}^{x} \sqrt{f_{\epsilon}} \mathrm{d} y\right|^{2},\left|\hat{\zeta}_{\epsilon}\right|^{3}:=\left|\frac{3}{2} \int_{\hat{x}_{0}(\epsilon)}^{x} \sqrt{f_{\epsilon}} \mathrm{d} y\right|^{2}$ and set

$$
\begin{aligned}
B\left(f_{\epsilon}, g_{\epsilon}\right)= & \left|\frac{f_{\epsilon}^{\prime}\left(x_{1}\right)}{f_{\epsilon}^{\frac{3}{2}}\left(x_{1}\right)}\right|+\left|\int_{x_{1}}^{x_{0}(\epsilon)} \sqrt{f_{\epsilon}}(x) \mathrm{d} x\right|^{-1}+\left|\int_{\hat{x}_{0}(\epsilon)}^{x_{1}} \sqrt{f_{\epsilon}}(x) \mathrm{d} x\right|^{-1} \\
& +\mathcal{V}_{x_{1},+\infty}\left(H_{\epsilon}\right)+\mathcal{V}_{-\infty, x_{1}}\left(\hat{H}_{\epsilon}\right) .
\end{aligned}
$$

Assume $B_{0}:=\sup _{\epsilon \in I_{0}} B\left(f_{\epsilon}, g_{\epsilon}\right)<+\infty$.

Then, for all $u$ sufficiently large and all $\epsilon \in I_{0}$, if the differential equation

$$
w^{\prime \prime}=\left(u^{2} f_{\epsilon}+g_{\epsilon}\right) w
$$


admits a bound state $w$ (i.e. a solution which is recessive at both ends $x \rightarrow$ $\pm \infty)$, then

$$
\frac{2}{\pi} \int_{\hat{x}_{0}(\epsilon)}^{x_{0}(\epsilon)} \sqrt{-f_{\epsilon}} \mathrm{d} x+\vartheta_{f_{\epsilon}, g_{\epsilon}, u, n}=\frac{2 n+1}{u}
$$

for some positive integer $n \in \mathbb{N}$ and for an error function $\vartheta_{f_{\epsilon}, g_{\epsilon}, u, n}$ which obeys $\left|\vartheta_{f_{\epsilon}, g_{\epsilon}, u, n}\right| \lesssim_{B_{0}} u^{-2}$. In particular, the implicit constant is independent of $\epsilon, u, n$.

Conversely, for all $u$ sufficiently large, there exists an error function $\vartheta_{f_{\epsilon}, g_{\epsilon}, u, n}$ which depends continuously on $\epsilon \in I_{0}$ and satisfies $\left|\vartheta_{f_{\epsilon}, g_{\epsilon}, u, n}\right| \lesssim B_{0}$ $u^{-2}$ such that (3.25) admits a bound state $w$ if there exist $a n \epsilon \in I_{0}$ and $a$ $n \in \mathbb{N}$ satisfying (3.26).

Proof The above result follows from [94, Chapter 13, §8.2] but for convenience of the reader we briefly outline the steps in [94, Chapter 13, §8.2] in our context. We begin by noting that our assumption $B_{0}<\infty$ shows that the error terms in (8.03) and (8.05) of [94, Chapter 13, §8.2] are controlled by $O_{B_{0}}\left(u^{-1}\right)$ uniformly for $u$ sufficiently large and $\epsilon \in I_{0}$. Following the argument of [94, Chapter 13, §8.2] we then conclude that for $u$ sufficiently large, (3.25) admits a bound state $w$ if and only if $\sin \left(u \int_{\hat{x}_{0}(\epsilon)}^{x_{0}(\epsilon)} \sqrt{-f_{\epsilon}} \mathrm{d} x-\frac{\pi}{2}\right)=\Theta\left(f_{\epsilon}, g_{\epsilon}, u\right)$, for some error function $\Theta$ which satisfies $\sup _{\epsilon \in I_{0}}\left|\Theta\left(f_{\epsilon}, g_{\epsilon}, u\right)\right| \lesssim_{B_{0}} u^{-1}$. By virtue of $f_{\epsilon}$ and $g_{\epsilon}$ depending continuously on $\epsilon$, we also obtain that $\Theta\left(f_{\epsilon}, g_{\epsilon}, u\right)$ depends continuously on $\epsilon$. Inverting sin around its zeros yields the claim.

With the above proposition in hand we proceed to the main proposition of this subsection, where we recall that we still consider the case $\omega=\omega_{-} m$.

Proposition 3.2 Let $\mathfrak{p}_{0} \in \mathscr{P}$ be arbitrary but fixed. Then, for almost every $\tilde{\lambda}_{0} \in\left(\Xi^{2}, \infty\right)$ (more precisely, for every $\tilde{\lambda}_{0} \in\left(\Xi^{2}, \infty\right) \backslash \mathcal{N}_{\mathfrak{p}_{0}}$ for some Lebesgue null set $\mathcal{N}_{\mathfrak{p}_{0}}$ ), there exists a strictly increasing sequence of natural numbers $\left(m_{i}\right)_{i \in \mathbb{N}}$ such that for every $i \in \mathbb{N}$, the operator $P_{\omega_{-}}$admits an eigenvalue $\lambda_{i}:=\lambda_{m_{i} \ell_{i}}=\lambda_{m_{i} \ell_{i}}\left(\omega=\omega_{-} m\right)$ satisfying

$$
\tilde{\lambda}_{i}:=\lambda_{i} m_{i}^{-2}=\tilde{\lambda}_{0}+\lambda_{\text {error }}^{(i)} m_{i}^{-2},
$$

where $\left|\lambda_{\text {error }}^{(i)}\right| \leq C\left(\tilde{\lambda}_{0}, \mathfrak{p}_{0}\right)$ as $m_{i} \rightarrow \infty$ for some constant $C\left(\tilde{\lambda}_{0}, \mathfrak{p}_{0}\right)>0$. Moreover, $m_{i} \leq \ell_{i} \leq m_{i}^{2}$.

Proof We consider the formulation of the angular o.d.e. in (3.9) and moreover change coordinates

$$
y(x)=\int_{0}^{x} \frac{1}{\Delta_{\tilde{x}}\left(1-\tilde{x}^{2}\right)} \mathrm{d} \tilde{x}
$$


such that

$$
\frac{\mathrm{d} x}{\mathrm{~d} y}=\Delta_{x}\left(1-x^{2}\right)
$$

This yields the equivalent eigenvalue problem

$$
-\frac{\mathrm{d}^{2}}{\mathrm{~d} y^{2}} g+\left(m^{2} W_{1}+P_{\text {error }}\right) g=0
$$

for $g$ in a dense domain of $L^{2}(\mathbb{R}, \mathrm{d} y)$. From Lmma 3.1 we have that $W_{1}$ has a unique positive root for $\tilde{\lambda}>\Xi^{2}$ which we denote with $y_{0}(\tilde{\lambda}):=y\left(x_{0}(\tilde{\lambda})\right)$. We also define

$$
\xi(\tilde{\lambda}):=\int_{-y_{0}(\tilde{\lambda})}^{y_{0}(\tilde{\lambda})} \sqrt{-W_{1}} \mathrm{~d} y
$$

where we recall that $W_{1}$ is symmetric around the origin. For the potential $W_{1}$, we have (e.g. [38, p. 118]) that $\xi:\left(\Xi^{2},+\infty\right) \rightarrow \mathbb{R}, \tilde{\lambda} \mapsto \xi(\tilde{\lambda})$ is a strictly increasing smooth (even real-analytic) function. Further note that

$$
\frac{\mathrm{d} \xi}{\mathrm{d} \tilde{\lambda}}=\int_{-y_{0}(\tilde{\lambda})}^{y_{0}(\tilde{\lambda})} \frac{\Delta_{x(y)}\left(1-x(y)^{2}\right)}{2 \sqrt{-W_{1}}} \mathrm{~d} y>0
$$

so by the inverse function theorem, $\xi$ has a smooth inverse.

By a standard result on Diophantine approximation (see e.g. [51, Corollary (ii) after Theorem 6.2]), we have that for each $x \in \mathbb{R}_{>0} \backslash \mathcal{N}$, where $\mathcal{N}$ is a Lebesgue null set, there exist sequences of natural numbers $\left(n_{i}\right)_{i \in \mathbb{N}}$ and $\left(m_{i}\right)_{i \in \mathbb{N}}$ with $n_{i+1}>n_{i}$ and $m_{i+1}>m_{i}$ such that

$$
\left|\frac{2}{\pi} x-\frac{2 n_{i}+1}{m_{i}}\right| \leq \frac{1}{m_{i}^{2}}
$$

for all $i \in \mathbb{N}$. Indeed, the assumptions of [51, Corollary (ii) after Theorem 6.2] are satisfied as $\sum_{m \in \mathbb{N}} \frac{1}{m}$ is divergent and $2 n+1=1(\bmod 2), m=0(\bmod 1)$, where $(1,2,0,1)$ is pairwise coprime, i.e. $(1,2,1,1)=1$ in the notation of [51, Corollary (ii) after Theorem 6.2]. Alternatively, the result also follows from [51, Theorem 6.6] after noting that the sum of the lower asymptotic densities $\underline{d}$ of the odd and the natural numbers exceeds 1 , i.e. $\underline{d}(\mathbb{N})+\underline{d}(2 \mathbb{N}+$ 1) $=1+\overline{\frac{1}{2}}>1$. 
Now, since $\xi$ has a smooth inverse, there exists a Lebesgue null set $\mathcal{N}_{\mathfrak{p}_{0}}:=$ $\xi^{-1}(\mathcal{N}) \subset\left(\Xi^{2}, \infty\right)$ such that for each $\tilde{\lambda}_{0} \in\left(\Xi^{2}, \infty\right) \backslash \mathcal{N}_{\mathfrak{p}_{0}}$ we have

$$
\left|\frac{2}{\pi} \xi\left(\tilde{\lambda}_{0}\right)-\frac{2 n_{i}+1}{m_{i}}\right| \leq \frac{1}{m_{i}^{2}}
$$

for a sequence of natural numbers $\left(n_{i}\right)_{i \in \mathbb{N}}$ and $\left(m_{i}\right)_{i \in \mathbb{N}}$ with $n_{i+1}>n_{i}$ and $m_{i+1}>m_{i}$.

Now, we will apply Proposition 3.1. For $\tilde{\lambda}_{0} \in\left(\Xi^{2}, \infty\right) \backslash \mathcal{N}_{\mathfrak{p}_{0}}$, choose a small neighborhood $\mathcal{U}_{\tilde{\lambda}_{0}}$ such that for all $\tilde{\lambda}$ in the closure of $U_{\tilde{\lambda}_{0}}$, we have $\tilde{\lambda}>\Xi^{2}$. We will now consider $\tilde{\lambda} \in \mathcal{U}_{\tilde{\lambda}_{0}}$ which will take the role of $\epsilon$ appearing in Proposition 3.1. We will now show that indeed the assumptions of Proposition 3.1 are satisfied. First, note that $W_{1}$ and $P_{\text {error }}$ are smooth for all $\tilde{\lambda} \in \mathcal{U}_{\tilde{\lambda}_{0}}$. Further, uniformly in $\mathcal{U}_{\tilde{\lambda}_{0}}, \int_{0}^{y} \sqrt{\left|W_{1}\right|} \mathrm{d} \tilde{y}$ diverges as $y \rightarrow \pm \infty$. Moreover, the potential $W_{1}$ has two simple roots which do not coalesce uniformly in $\mathcal{U}_{\tilde{\lambda}_{0}}$ in view of Lmma 3.1. In particular, this also shows that in a region $[0, c]$ for any fixed $c>0$ (in particular containing the right turning point), the total variation of $H_{\tilde{\lambda}}$ is bounded uniformly in $\mathcal{U}_{\tilde{\lambda}_{0}}$; analogously for $\hat{H}_{\tilde{\lambda}}$ in $[-c, 0]$. Remark from [94, Chapter 11, §3] that indeed (3.22) and (3.23) are by construction the quantitative versions of the qualitative statement of two non-coalescing roots. To show that $\mathcal{V}_{0,+\infty}\left(H_{\tilde{\lambda}}\right)$ and $\mathcal{V}_{-\infty, 0}\left(\hat{H}_{\tilde{\lambda}}\right)$ remain bounded at $\pm \infty$, respectively, we note that for $|y| \rightarrow \infty$, we have

$$
\frac{\Xi^{2}}{2} \leq W_{1} \leq \Xi^{2}, \quad \text { and } \quad\left|\frac{\mathrm{d} W_{1}}{\mathrm{~d} y}\right|,\left|\frac{\mathrm{d}^{2} W_{1}}{\mathrm{~d} y^{2}}\right| \lesssim \frac{\mathrm{d} x}{\mathrm{~d} y}
$$

as well as

$$
\left|P_{\text {error }}\right| \lesssim \frac{\mathrm{d} x}{\mathrm{~d} y}
$$

Inserting these bounds in (3.22) and (3.23) we obtain

$$
\mathcal{V}_{0,+\infty}\left(H_{\tilde{\lambda}}\right)+\mathcal{V}_{-\infty, 0}\left(\hat{H}_{\tilde{\lambda}}\right) \lesssim 1
$$

uniformly in $\mathcal{U}_{\tilde{\lambda}_{0}}$. The others bounds of (3.24) (uniformly in $\mathcal{U}_{\tilde{\lambda}_{0}}$ ) also follow directly from the previous estimates and we obtain $B_{0}=\sup _{\tilde{\lambda} \in \mathcal{U}_{\tilde{\lambda}_{0}}} \in$ $B\left(W_{1}, P_{\text {error }}\right) \lesssim 1$. Thus, from Proposition 3.1 we now conclude that the eigenvalues $\lambda=\tilde{\lambda} m^{2}$ for $\tilde{\lambda}$ in a neighborhood of $\tilde{\lambda}_{0}$ are characterized by

$$
\frac{2}{\pi} \xi(\tilde{\lambda})+\vartheta_{\tilde{\lambda}_{0}, m, n, B\left(W_{1}, P_{\text {error }}\right)}=\frac{2 n+1}{m}
$$


for $n \in \mathbb{N}$, where $\left|\vartheta_{\tilde{\lambda}_{0}, m, n, B\left(W_{1}, P_{\text {error }}\right)}\right| \lesssim \tilde{\lambda}_{0} m^{-2}$.

Now, for fixed $\tilde{\lambda}_{0} \in\left(\Xi^{2}, \infty\right) \backslash \mathcal{N}_{\mathfrak{p}_{0}}$, let the sequence $\left(m_{i}, n_{i}\right)_{i \in \mathbb{N}}$ as above be such that (3.34) holds. Then, we obtain associated eigenvalues from (3.38) which satisfy

$$
\begin{aligned}
\tilde{\lambda}_{i} & =\xi^{-1}\left(\frac{\pi}{2} \frac{2 n_{i}+1}{m_{i}}-\frac{\pi}{2} \vartheta_{\tilde{\lambda}_{0}, m_{i}, n_{i}, B\left(W_{1}, P_{\text {error }}\right)}\right) \\
& =\xi^{-1}\left(\xi\left(\tilde{\lambda}_{0}\right)+O_{\tilde{\lambda}_{0}}\left(m_{i}^{-2}\right)\right)=\tilde{\lambda}_{0}+O_{\tilde{\lambda}_{0}}\left(m_{i}^{-2}\right) .
\end{aligned}
$$

The last equality holds due Taylor's theorem and (3.32).

\subsection{Bounds on $\partial_{\xi} \lambda_{m \ell}$ and $\partial_{\xi} S_{m \ell}$ near interior scattering poles}

In the proof of Theorem 1 in Sect. 9 we will need to control the quantities $\partial_{\omega} \lambda_{m \ell}(a \omega)$ and $\partial_{\omega} S_{m \ell}(a \omega)$ near the interior scattering poles, i.e. for $\omega \approx \omega \_m$. We will choose our initial data in Sect. 6 to be supported on angular modes $m>0$ which are large and positive. Thus, for the rest of this subsection, we assume that $m>0$ and think of $1 / m$ as a semiclassical parameter. We first note that $\xi \mapsto S_{m \ell}(\xi, x)$ is smooth as $\xi$ is a smooth parameter of the angular o.d.e. (2.42) solved by $S_{m \ell}$. Now, a direct computation shows that

$$
\partial_{\xi} S_{m \ell}=\frac{\partial S_{m \ell}(\xi, x)}{\partial \xi}
$$

solves the inhomogeneous o.d.e.

$$
\left(P_{x}-\lambda_{m \ell}\right) \partial_{\xi} S_{m \ell}=\left(\partial_{\xi} P_{x}-\partial_{\xi} \lambda_{m \ell}\right) S_{m \ell}
$$

with Dirichlet boundary conditions at $x= \pm 1$, where

$$
\partial_{\xi} P_{x}=\frac{\partial P_{x}(\xi)}{\partial \xi}=-2 \Xi \xi \frac{x^{2}}{\Delta_{x}}-2 m \frac{\Xi}{\Delta_{x}} \frac{a^{2}}{l^{2}} x^{2} .
$$

We will first consider $\partial_{\xi} \lambda_{m \ell}$.

Lemma 3.2 The eigenvalues $\lambda_{m \ell}(\xi)$ of $P_{x}(\xi)$ as in (3.1) satisfy

$$
\left|\partial_{\xi} \lambda_{m \ell}(\xi)\right| \leq\left|\left\langle S_{m \ell}, \partial_{\xi} P_{x} S_{m \ell}\right\rangle_{L^{2}(-1,1)}\right|
$$

and thus,

$$
\sup _{\xi \in\left(a m \omega_{-}-\frac{1}{m}, a m \omega_{-}+\frac{1}{m}\right)}\left|\partial_{\xi} \lambda_{m \ell}(\xi)\right| \lesssim|m|
$$


Proof Taking the $L^{2}$-inner product of (3.41) with $S_{m \ell}$ and using that $P_{x}$ is self-adjoint, shows that

$$
\left\langle S_{m \ell},\left(\partial_{\xi} P_{x}-\partial_{\xi} \lambda_{m \ell}\right) S_{m \ell}\right\rangle_{L^{2}(-1,1)}=0
$$

from which we obtain

$$
\left|\partial_{\xi} \lambda\right| \leq\left|\left\langle S_{m \ell}, \partial_{\xi} P_{x} S_{m \ell}\right\rangle_{L^{2}(-1,1)}\right| \leq\left\|\partial_{\xi} P_{x}\right\|
$$

in view of $\left\langle S_{m \ell}, S_{m \ell}\right\rangle_{L^{2}(-1,1)}=1$. Here $\left\|\partial_{\xi} P_{x}\right\|$ denotes the operator norm which is equal to the $L^{\infty}$ norm as $\partial_{\xi} P_{x}$ is a multiplication operator (see (3.42)). Now, the claim follows from the fact that $\left\|\partial_{\xi} P_{x}\right\|_{L^{\infty}} \lesssim|\xi|+|m|$.

It is more difficult to obtain estimates for $\partial_{\xi} S_{m \ell}$ which we express as

$$
\partial_{\xi} S_{m \ell}=\operatorname{Res}\left(\lambda_{m \ell} ; P_{x}\right) \Pi_{S_{m \ell}}^{\perp} H,
$$

where

$$
H=\left(\partial_{\xi} P_{x}-\partial_{\xi} \lambda_{m \ell}\right) S_{m \ell}
$$

is the inhomogeneous term of (3.41), $\operatorname{Res}\left(\lambda ; P_{x}\right)$ is the resolvent and $\Pi_{S_{m \ell}}^{\perp}$ is the orthogonal projection on the orthogonal complement of $S_{m \ell}$. At this point we also remark that both $\partial_{\xi} S_{m \ell}$ and $H$ are orthogonal to $S_{m \ell}$ which follows from $\xi \mapsto\left\langle S_{m \ell}, S_{m \ell}\right\rangle_{L^{2}(-1,1)}=1$ and (3.45), respectively.

A possible way to control the resolvent operator $\operatorname{Res}\left(\lambda_{m \ell} ; P_{x}\right) \Pi_{S_{m \ell}}^{\perp}$ is to show lower bounds on the spectral gaps $\left|\lambda_{m, \ell}(a \omega)-\lambda_{m, \ell+1}(a \omega)\right|$ uniformly in $m, \ell \rightarrow \infty$ and $\omega \approx \omega_{-} m$. Our approach is based on an explicit construction of the resolvent kernel via suitable approximations with parabolic cylinder functions and Airy functions.

We begin by noting that from standard results on solutions to SturmLiouville problems, each eigenfunction $S_{m \ell}$ is either symmetric or antisymmetric around $x=0$. If $S_{m \ell}$ is antisymmetric around $x=0$ we have $S_{m \ell}(x=0)=0$, i.e. Dirichlet boundary conditions at $x=0$. Analogously, if $S_{m \ell}$ is symmetric, we have Neumann boundary conditions at $x=0$, i.e. $\frac{\mathrm{d}}{\mathrm{d} x} S_{m \ell}(x=0)=0$. Also note that $\partial_{\xi} S_{m \ell}$ inherits the symmetry properties of $S_{m \ell}$. Hence, the problem reduces to studying the interval $x \in[0,1)$ with Dirichlet/Neumann boundary conditions at $x=0$ and Dirichlet boundary conditions at $x=1$. In view of the above, $\partial_{\xi} S_{m \ell}$ will satisfy

$$
\partial_{\xi} S_{m \ell}(x=0, \xi)=0 \quad \text { or } \quad \frac{\mathrm{d}}{\mathrm{d} x} \partial_{\xi} S_{m \ell}(x=0, \xi)=0
$$


depending on $S_{m \ell}(x=0)=0$ or $\frac{\mathrm{d}}{\mathrm{d} x} S_{m \ell}(x=0)=0$, respectively, as well as

$$
\partial_{\xi} S_{m \ell}(x=1, \xi)=0 .
$$

In addition to satisfying the above boundary conditions, $\partial_{\xi} S_{m \ell}$ is also a solution of the inhomogenous o.d.e. (3.41) which we explicitly write out as

$$
\begin{aligned}
{[-} & \frac{\mathrm{d}}{\mathrm{d} x}\left(\Delta_{x}\left(1-x^{2}\right) \frac{\mathrm{d}}{\mathrm{d} x} \cdot\right)+\frac{\Xi^{2} m^{2}}{\Delta_{x}\left(1-x^{2}\right)} \\
& -\Xi m^{2} a^{2} \omega_{-}^{2} \frac{x^{2}}{\Delta_{x}}-2 m^{2} a \omega_{-} \frac{\Xi}{\Delta_{x}} \frac{a^{2}}{l^{2}} x^{2} \\
& \left.+\frac{2}{l^{2}} a^{2}\left(1-x^{2}\right)-\Xi\left(2 \epsilon a \omega_{-}+\epsilon^{2} m^{-2}\right) \frac{x^{2}}{\Delta_{x}}-2 \epsilon \frac{\Xi}{\Delta_{x}} \frac{a^{2}}{l^{2}} x^{2}-\lambda_{m \ell}\right] \partial_{\xi} S_{m \ell} \\
= & {\left[\partial_{\xi} \lambda_{m \ell}+2 \Xi\left(a m \omega_{-}+\frac{\epsilon}{m}\right) \frac{x^{2}}{\Delta_{x}}+2 m \frac{\Xi}{\Delta_{x}} \frac{a^{2}}{l^{2}} x^{2}\right] S_{m \ell}, }
\end{aligned}
$$

where $|\epsilon|<1$ is such that $\xi=a m \omega_{-}+\frac{\epsilon}{m}$. Moreover, $\partial_{\xi} S_{m \ell}$ and $H$ admit the same symmetries as $S_{m \ell}$ such that, both $H$ and $\partial_{\xi} S_{m \ell}$ are orthogonal to $S_{m \ell}$ in $L^{2}([0,1))$. Also recall that

$$
\left\langle S_{m \ell}, S_{m \ell}\right\rangle_{L^{2}(-1,1)}=\int_{-1}^{1} S_{m \ell}^{2} \mathrm{~d} x=1
$$

such that

$$
\int_{0}^{1} S_{m \ell}^{2} \mathrm{~d} x=\frac{1}{2}
$$

As in the proof of Proposition 3.2, we introduce the variable $y=y(x)$ through the conditions

$$
y(0)=0, \frac{\mathrm{d} y}{\mathrm{~d} x}=\frac{1}{\Delta_{x}\left(1-x^{2}\right)}
$$

as well as the associated Hilbert space $L^{2}([0, \infty), w(y) \mathrm{d} y)$, where

$$
w(y)=\Delta_{x(y)}\left(1-x(y)^{2}\right) .
$$

This can be computed explicitly as

$$
y(x)=\frac{1}{2 \Xi}\left(\log (1+x)-\log (1-x)+\frac{a}{l} \log \left(1-\frac{a}{l} x\right)-\frac{a}{l} \log \left(1+\frac{a}{l} x\right)\right) .
$$


Note that

$$
e^{2 \Xi y}=\frac{1+x}{1-x}\left(\frac{1-\frac{a}{l} x}{1+\frac{a}{l} x}\right)^{\frac{a}{l}}
$$

In this new variable, we define

$$
s_{1}(y):=S_{m \ell}(x(y)) \text { and } s_{p}(y):=\partial_{\xi} S_{m \ell}(x(y))
$$

such that

$$
\int_{0}^{\infty} s_{1}^{2}(y) \Delta_{x}\left(1-x^{2}(y)\right) \mathrm{d} y=\int_{0}^{1} S_{m \ell}^{2} \mathrm{~d} x=\frac{1}{2} .
$$

Then, we re-write (3.51) as

$$
\begin{aligned}
& -\frac{\mathrm{d}^{2}}{\mathrm{~d} y^{2}} s_{p}+m^{2}\left(\Xi^{2}-\left[\Xi a^{2} \omega_{-}^{2}+2 a \omega_{-} \Xi \frac{a^{2}}{l^{2}}\right] x^{2}\left(1-x^{2}\right)-\tilde{\lambda} \Delta_{x}\left(1-x^{2}\right)\right) s_{p} \\
& \quad+\Delta_{x}\left(1-x^{2}\right)\left(\frac{2}{l^{2}} a^{2}\left(1-x^{2}\right)-\Xi\left(2 \epsilon \omega_{-}+\epsilon^{2} m^{-2}\right) \frac{x^{2}}{\Delta_{x}}-2 \epsilon \frac{\Xi}{\Delta_{x}} \frac{a^{2}}{l^{2}} x^{2}\right) s_{p} \\
& =\Delta_{x}\left(1-x^{2}\right)\left[\partial_{\xi} \lambda+2 \Xi\left(a m \omega_{-}+\frac{\epsilon}{m}\right) \frac{x^{2}}{\Delta_{x}}+2 m \frac{\Xi}{\Delta_{x}} \frac{a^{2}}{l^{2}} x^{2}\right] s_{1} .
\end{aligned}
$$

We recall the definition of $W_{1}$ in (3.12) as

$$
\begin{aligned}
W_{1}(x(y))= & \Xi^{2}-\left[\Xi a^{2} \omega_{-}^{2}+2 a \omega_{-} \Xi \frac{a^{2}}{l^{2}}\right] x(y)^{2}\left(1-x(y)^{2}\right) \\
& -\tilde{\lambda} \Delta_{x(y)}\left(1-x(y)^{2}\right),
\end{aligned}
$$

and define

$$
\begin{aligned}
W_{2}(x(y)):= & w(y)\left(\frac{2}{l^{2}} a^{2}\left(1-x(y)^{2}\right)-\Xi\left(2 \epsilon a \omega_{-}+\epsilon^{2} m^{-2}\right) \frac{x(y)^{2}}{\Delta_{x(y)}}\right. \\
& \left.-2 \epsilon \frac{\Xi}{\Delta_{x(y)}} \frac{a^{2}}{l^{2}} x(y)^{2}\right)
\end{aligned}
$$

as well as

$$
F(x(y)):=w(y)\left(\partial_{\xi} \lambda+2 \Xi\left(a m \omega_{-}+\frac{\epsilon}{m}\right) \frac{x(y)^{2}}{\Delta_{(y)}}+2 m \frac{\Xi}{\Delta_{x(y)}} \frac{a^{2}}{l^{2}} x(y)^{2}\right) .
$$


Thus, (3.60) reads

$$
-\frac{\mathrm{d}^{2}}{\mathrm{~d} y^{2}} s_{p}+\left(m^{2} W_{1}+W_{2}\right) s_{p}=F s_{1}
$$

where we recall that $s_{p}$ satisfies Dirichlet/Neumann boundary conditions at $y=0$ and vanishes at $y=+\infty$. We also note that the previous orthogonality properties remain, i.e. both $s_{p}$ and $w^{-1} F s_{1}$ are orthogonal to $s_{1}$ in the Hilbert space $L^{2}([0, \infty), w(y) \mathrm{d} y)$.

In order to construct the resolvent operator, we will first state the existence of a further suitable solution $s_{2}$ to the homogeneous equation

$$
-\frac{\mathrm{d}^{2}}{\mathrm{~d} y^{2}} g+\left(m^{2} W_{1}+W_{2}\right) g=0
$$

which is linearly independent from $s_{1}$. This is the content of the following lemma which will be proved in Sect. 3.4.

Lemma 3.3 Let $m \in \mathbb{N}$ sufficiently large as in Sect. 3.4. For $\xi \in\left(a \omega_{-} m-\right.$ $\left.\frac{1}{m}, a \omega_{-} m+\frac{1}{m}\right)$, there exists a solution $s_{2}$ to (3.65) with $\mathfrak{W}\left(s_{1}, s_{2}\right)=1$. Moreover, $g_{p}$ defined as

$$
g_{p}(y):=s_{2}(y) \int_{y}^{\infty} s_{1}^{2}(\tilde{y}) F(\tilde{y}) \mathrm{d} \tilde{y}+s_{1}(y) \int_{0}^{y} s_{2}(\tilde{y}) s_{1}(\tilde{y}) F(\tilde{y}) \mathrm{d} \tilde{y}
$$

satisfies

$$
\left\|g_{p}\right\|_{L^{2}([0, \infty), w(y) \mathrm{d} y)}^{2}=\int_{0}^{\infty} g_{p}(y)^{2}\left(1-x(y)^{2}\right) \Delta_{x} \mathrm{~d} y \lesssim m .
$$

Proof This is proved in Sect. 3.4, more specifically the claim follows from Lemma 3.13 and Lemma 3.18.

With $s_{2}$ in hand we will now construct the integral kernel of the resolvent $\operatorname{Res}\left(\lambda_{m \ell} ; P_{x}\right) \Pi_{S_{m \ell}}^{\perp}$ in $y$-coordinates. More specifically, we show

Lemma 3.4 The solution $s_{p}(y)=\partial_{\xi} S_{m \ell}(x(y))=\operatorname{Res}\left(\lambda_{m \ell} ; P_{x}\right) \Pi_{S_{m \ell}}^{\perp}(H)(x(y))$ of (3.64) satisfies

$$
\begin{aligned}
s_{p}(y)= & g_{p}(y)+c_{p 1} s_{1}(y)=s_{2}(y) \int_{y}^{\infty} s_{1}^{2}(\tilde{y}) F(\tilde{y}) \mathrm{d} \tilde{y} \\
& +s_{1}(y)\left(\int_{0}^{y} s_{2}(\tilde{y}) s_{1}(\tilde{y}) F(\tilde{y}) \mathrm{d} \tilde{y}+c_{p 1}\right),
\end{aligned}
$$


for some constant $c_{p 1} \in \mathbb{R}$.

Proof Since $s_{p}$ is a solution of the inhomogeneous o.d.e. (3.64), it can be written (using $\mathfrak{W}\left(s_{1}, s_{2}\right)=1$ ) as

$$
\begin{aligned}
s_{p}(y)= & s_{2}(y)\left(\int_{y}^{\infty} s_{1}^{2}(\tilde{y}) F(\tilde{y}) \mathrm{d} \tilde{y}+c_{p 2}\right) \\
& +s_{1}(y)\left(\int_{0}^{y} s_{2}(\tilde{y}) s_{1}(\tilde{y}) F(\tilde{y}) \mathrm{d} \tilde{y}+c_{p 1}\right)
\end{aligned}
$$

for some constants $c_{p 1}, c_{p 2} \in \mathbb{R}$. It remains to show that $c_{p 2}=0$ and we consider the cases of Dirichlet/Neumann conditions of $s_{1}$ at $y=0$ independently.

First, assume that $s_{1}(y=0)=0$, then we also have that $s_{p}(y=0)=0$ (see (3.49)). Evaluating the right hand side of (3.69) at $y=0$ we obtain

$$
\begin{aligned}
& s_{2}(0)\left(\int_{0}^{\infty} s_{1}^{2}(\tilde{y}) F(\tilde{y}) \mathrm{d} \tilde{y}+c_{p 2}\right)+s_{1}(0)\left(\int_{0}^{0} s_{2}(\tilde{y}) s_{1}(\tilde{y}) F(\tilde{y}) \mathrm{d} \tilde{y}+c_{p 1}\right) \\
& =s_{2}(0)\left(\int_{0}^{\infty} s_{1}^{2}(\tilde{y}) F(\tilde{y}) \mathrm{d} \tilde{y}+c_{p 2}\right)=s_{2}(0) c_{p 2},
\end{aligned}
$$

where we have used that $s_{1}(y=0)=0$ and that $s_{1}$ is $L^{2}([0, \infty), w(y) \mathrm{d} y)$ orthogonal to $w^{-1} s_{1} F$. Moreover, from the Wronskian condition $\mathfrak{W}\left(s_{1}, s_{2}\right)=$ 1 we have that $s_{2}(y=0) \neq 0$. Thus, $c_{p 2}=0$ follows from $s_{p}(y=0)=0$.

Now, if $s_{1}$ satisfies the Neumann condition $\frac{\mathrm{d}}{\mathrm{d} y} s_{1}(y=0)=0$, then so does $s_{p}$, i.e. $\frac{\mathrm{d}}{\mathrm{d} y} s_{p}(y=0)=0$. Differentiating the right hand side of (3.69) and evaluating this at $y=0$ yields

$$
\frac{\mathrm{d}}{\mathrm{d} y} s_{2}(0) c_{p 2}-s_{2}(0) s_{1}^{2}(0) F(0)+s_{1}(0)^{2} s_{2}(0) F(0)=\frac{\mathrm{d}}{\mathrm{d} y} s_{2}(0) c_{p 2} .
$$

From the Wronskian identity we again have that $\frac{\mathrm{d}}{\mathrm{d} y} s_{2}(0) \neq 0$ such that $c_{p 2}=0$ follows from $\frac{\mathrm{d}}{\mathrm{d} y} s_{p}(y=0)=0$.

Up to the completion of the proof of Lemma 3.3, which is the content of Sect. 3.4, we will now show the main proposition of this subsection.

Proposition 3.3 For all $m \in \mathbb{N}$ sufficiently large, the eigenfunctions $S_{m \ell}(\xi, \cos \theta)$ of the operator $P$ defined in (2.42) satisfy

$$
\sup _{\omega \in\left(\omega-m-\frac{1}{a m}, \omega-m+\frac{1}{a m}\right)}\left\|\partial_{\omega} S_{m \ell}(a \omega, \cdot)\right\|_{L^{2}([0, \pi] ; \sin \theta \mathrm{d} \theta)} \lesssim m^{\frac{1}{2}} .
$$


Proof First note that $\xi=a \omega$ such that $\partial_{\omega}=a \partial_{\xi}$. Then, we have

$$
\begin{aligned}
\left\|\partial_{\xi} S_{m \ell}\right\|_{L^{2}(-1,1)}^{2} & =\left\|\operatorname{Res}\left(\lambda_{m \ell} ; P_{x}\right) \Pi_{S_{m \ell}}^{\perp} H\right\|_{L^{2}(-1,1)}^{2}=2\left\|s_{p}\right\|_{L^{2}([0, \infty), w(y) \mathrm{d} y)}^{2} \\
& =2\left\|\Pi_{s_{1}}^{\perp} g_{p}\right\|_{L^{2}([0, \infty), w(y) \mathrm{d} y)}^{2} \leq 2\left\|g_{p}\right\|_{L^{2}([0, \infty), w(y) \mathrm{d} y)}^{2},
\end{aligned}
$$

where we have used that

$$
s_{p}=\Pi_{s_{1}}^{\perp} g_{p}
$$

Here, $\Pi_{s_{1}}^{\perp}$ is the projection on the orthogonal complement of $s_{1}$ in $L^{2}([0, \infty), w(y) \mathrm{d} y)$. The estimate (3.72) follows now from (3.73) and Lemma 3.3.

\subsection{Semi-classical resolvent estimates near interior scattering poles}

Throughout this subsection (Sect. 3.4) we assume that

$$
\xi \in\left(a \omega \_m-\frac{1}{m}, a \omega_{-} m+\frac{1}{m}\right)
$$

and $m>0$. The goal of this subsection is to show Lemma 3.3. We first argue that for sufficiently large $m$, we only need to consider the case $\tilde{\lambda}>\Xi^{2}$ as all eigenvalues $\lambda_{m \ell}\left(a \omega_{-} m\right)$ at the interior scattering poles are larger than $\Xi^{2} m^{2}$.

Lemma 3.5 For sufficiently large $m$, we have inf $y \in \mathbb{R}\left(m^{2} W_{1}(y)+W_{2}(y)\right)>$ 0 for any $\tilde{\lambda} \leq \Xi^{2}$.

Proof By monotonicity of $W_{1}$ with respect to $\tilde{\lambda}$, it suffices to show the result for $\tilde{\lambda}=\Xi^{2}$. We recall from the definition of $W_{2}$ in (3.62) that $W_{2}$ is uniformly bounded and satisfies

$$
W_{2}(x=0)=\frac{2 a^{2}}{l^{2}}>0 .
$$

Since $W_{1} \geq 0$ in view of Lmma 3.1, we have positivity in a neighborhood $U$ around $y=0$, i.e. $\inf _{y \in U}\left(m^{2} W_{1}(y)+W_{2}(y)\right)>0$. Outside that neighborhood, in view of Lmma 3.1, we have that inf $y \in \mathbb{R} \backslash U W_{1}(y)>0$. To conclude we use that $W_{2}$ is uniformly bounded and the claim follows for all $m$ sufficiently large.

Lemma 3.6 For $\xi$ as in (3.75) and for sufficiently large $m$ as in Lemma 3.5, any eigenvalue $\lambda_{m \ell}(\xi)=m^{2} \tilde{\lambda}$ of $P_{x}$ satisfies $\tilde{\lambda}>\Xi^{2}$. 
Proof This is immediate as for $\tilde{\lambda} \leq \Xi^{2}$ and sufficiently large $m$, the operator $-\frac{\mathrm{d}^{2}}{\mathrm{~d} y^{2}}+m^{2} W_{1}+W_{2}$ is strictly positive in view of Lemma 3.5.

Thus, it suffices to show Lemma 3.3 for $\tilde{\lambda}>\Xi^{2}$ and we consider the case $\tilde{\lambda} \in\left(\Xi^{2}, \Xi^{2}+1\right]$ in Sect. 3.4.1 and the case $\tilde{\lambda} \in\left(\Xi^{2}+1, \infty\right)$ in Sect. 3.4.2.

\subsubsection{The case $\Xi^{2}<\tilde{\lambda} \leq \Xi^{2}+1$}

Let $\tilde{\lambda} \in\left(\Xi^{2}, \Xi^{2}+1\right]$. In this range, $\tilde{\lambda}$ can be arbitrarily close to $\Xi^{2}$. As $\tilde{\lambda} \rightarrow \Xi^{2}$, the root $y_{0}>0$ of the potential $W_{1}(y)$ coalesces with $y=0$. Thus, our estimates need to be uniform in this limit and the appropriate approximation will be given by parabolic cylinder functions. To do so we will introduce the following Liouville transform which is motivated by [93]. We define a new variable $^{4}$

$$
\xi=\xi(y)
$$

uniquely through the conditions

$$
\left(\frac{\mathrm{d} \xi}{\mathrm{d} y}\right)^{2}=\frac{W_{1}(y)}{\xi^{2}-\alpha^{2}} \text { for } y \neq y_{0}
$$

$\xi\left(y_{0}\right)=\alpha>0$ and $\xi(y=0)=0$. By construction, this defines $\xi=\xi(y)$ as a smooth (even real-analytic) increasing function with values in $[0, \infty)$, see also [93, Sect. 2.2]. Note that this holds true as the right hand side satisfies

$$
\frac{W_{1}(y)}{\xi^{2}-\alpha^{2}}>0
$$

for $y>0$. Equivalently, the function $\xi(y)$ can be expressed as

$$
\begin{aligned}
& \int_{y}^{y_{0}}\left(-W_{1}\right)^{\frac{1}{2}} \mathrm{~d} \tilde{y}=\int_{\xi(y)}^{\alpha}\left(\alpha^{2}-\tau^{2}\right)^{\frac{1}{2}} \mathrm{~d} \tau \text { for } y \leq y_{0}, \\
& \int_{y_{0}}^{y} W_{1}^{\frac{1}{2}} \mathrm{~d} \tilde{y}=\int_{\alpha}^{\xi(y)}\left(\tau^{2}-\alpha^{2}\right)^{\frac{1}{2}} \mathrm{~d} \tau \text { for } y_{0} \leq y<\infty .
\end{aligned}
$$

We also consider $y=y(\xi)$ as a function $\xi$ and define

$$
\sigma_{1}:=\left(\frac{\mathrm{d} y}{\mathrm{~d} \xi}\right)^{-\frac{1}{2}} s_{1}
$$

\footnotetext{
$\overline{4}$ Here and in the following, $\xi$ is not to be mixed up with $\xi$ appearing in (3.1).
} 
where we recall that $s_{1}$ was defined in (3.58). In this new variable $\xi$, the function $\sigma_{1}=\sigma_{1}(\xi)$ satisfies

$$
-\frac{\mathrm{d}^{2} \sigma}{\mathrm{d} \xi^{2}}+\left[m^{2}\left(\xi^{2}-\alpha^{2}\right)+\Psi\right] \sigma=0
$$

where the error function $\Psi$ is given by

$$
\Psi=\left(\frac{\mathrm{d} y}{\mathrm{~d} \xi}\right)^{2} W_{2}+\left(\frac{\mathrm{d} y}{\mathrm{~d} \xi}\right)^{\frac{1}{2}} \frac{\mathrm{d}^{2}}{\mathrm{~d} \xi^{2}}\left(\frac{\mathrm{d} y}{\mathrm{~d} \xi}\right)^{-\frac{1}{2}} .
$$

Since $W_{1}$ is analytic and non-increasing in $\tilde{\lambda}$, we apply [93, Lemma 1] to conclude that $\Psi$ is continuous for $(\xi, \alpha) \in[0, \infty) \times[0, A]$, where $A=$ $\xi\left(y_{0}\left(\tilde{\lambda}=\Xi^{2}+1\right)\right)$. Now, we define the error-control function (see (6.3) of [93])

$$
F_{1}:=\int_{0}^{\xi} \frac{|\Psi|}{\Omega(\xi \sqrt{2 m})} \mathrm{d} \xi
$$

with $\Omega(x)=|x|^{\frac{1}{3}}$. We will now bound the total variation of the error-control function $F_{1}$ in (3.85). To do so we first show

Lemma 3.7 The smooth and monotonic functions $\xi=\xi(y)$ and $y=y(\xi)$ as defined in (3.78) satisfy

$$
\begin{aligned}
& \xi^{2}(y) \sim y, \\
& \frac{\mathrm{d} y}{\mathrm{~d} \xi} \sim \xi \\
& \left|\frac{\mathrm{d}^{2} y}{\mathrm{~d} \xi^{2}}\right| \lesssim 1, \\
& \left|\frac{\mathrm{d}^{3} y}{\mathrm{~d} \xi^{3}}\right| \lesssim \xi^{-1}
\end{aligned}
$$

for all $\xi$ sufficiently large.

Proof We estimate

$$
\frac{\mathrm{d} \xi}{\mathrm{d} y} \lesssim \sqrt{\frac{\Xi^{2}}{\xi^{2}-\alpha^{2}}} \lesssim \frac{1}{\xi}
$$


for all $\xi$ large enough, where we have used that $W_{1} \sim \Xi^{2}$ for large $\xi$. Similarly,

$$
\frac{\mathrm{d} \xi}{\mathrm{d} y} \gtrsim \frac{1}{\xi}
$$

for $\xi$ large which shows (3.87). Upon integrating the inequalities, we obtain (3.86).

For (3.88), we differentiate (3.78) to obtain

$$
\left|\frac{\mathrm{d}^{2} y}{\mathrm{~d} \xi^{2}}\right|=\left|\frac{\mathrm{d}}{\mathrm{d} \xi} \sqrt{\frac{\xi^{2}-\alpha^{2}}{W_{1}(y(\xi))}}\right| \lesssim \sqrt{\frac{W_{1}}{\xi^{2}-\alpha^{2}}}\left|\frac{\xi}{W_{1}}+\frac{\xi^{2}}{W_{1}^{2}} \frac{\mathrm{d} W_{1}}{\mathrm{~d} x} \frac{\mathrm{d} x}{\mathrm{~d} y} \frac{\mathrm{d} y}{\mathrm{~d} \xi}\right| \lesssim 1,
$$

where we have used that

$$
W_{1} \sim 1, \quad \frac{\mathrm{d} W_{1}}{\mathrm{~d} x} \lesssim 1, \quad \frac{\mathrm{d} x}{\mathrm{~d} y} \lesssim e^{-2 \Xi y}, \quad \text { and } \frac{\mathrm{d} y}{\mathrm{~d} \xi} \lesssim \xi
$$

for $\xi$ large enough. In particular, it follows that $\left|\frac{\mathrm{d} W_{1}}{\mathrm{~d} \xi}\right| \lesssim e^{-\xi}$ and similarly that $\left|\frac{\mathrm{d}^{2} W_{1}}{\mathrm{~d} \xi^{2}}\right| \lesssim e^{-\xi}$.

Finally, we proceed to (3.89) by estimating for large $\xi$

$$
\begin{aligned}
\left|\frac{\mathrm{d}^{3} y}{\mathrm{~d} \xi^{3}}\right| & =\left|\frac{\mathrm{d}^{2}}{\mathrm{~d} \xi^{2}} \sqrt{\frac{\xi^{2}-\alpha^{2}}{W_{1}(y(\xi))}}\right|=\left|\frac{\mathrm{d}}{\mathrm{d} \xi}\left(\frac{\xi}{\sqrt{W_{1}\left(\xi^{2}-\alpha^{2}\right)}}-\frac{\sqrt{\xi^{2}-\alpha^{2}}}{2 W_{1}^{\frac{3}{2}}} \frac{\mathrm{d} W_{1}}{\mathrm{~d} \xi}\right)\right| \\
& \lesssim\left|\frac{1}{\sqrt{W_{1}\left(\xi^{2}-\alpha^{2}\right)}}\right|+\frac{\left|\frac{\mathrm{d} W_{1}}{\mathrm{~d} \xi} \xi^{2}\right|+\left|W_{1} \xi\right|}{\left|W_{1}\left(\xi^{2}-\alpha^{2}\right)\right|^{\frac{3}{2}}}+\left|\frac{\xi \frac{\mathrm{d}^{2} W_{1}}{\mathrm{~d} \xi^{2}}}{W_{1}^{\frac{3}{2}}}\right|+\left|\frac{\frac{\mathrm{d} W_{1}}{\mathrm{~d} \xi}}{W_{1}^{\frac{3}{2}}}\right| \\
& +\left|\frac{\xi}{W_{1}^{\frac{5}{2}}}\left(\frac{\mathrm{d} W_{1}}{\mathrm{~d} \xi}\right)^{2}\right| \lesssim \xi^{-1}
\end{aligned}
$$

in view of the above estimates.

This allows us now to estimate the total variation of the error control function $F_{1}$.

Lemma 3.8 The error control function $F_{1}$ as defined in (3.85) satisfies

$$
\mathcal{V}_{0, \infty}\left(F_{1}\right) \lesssim \frac{1}{m^{\frac{1}{6}}}
$$


Proof As $\Psi$ is continuous on $[0, \infty)$, it suffices to control the integral for large $\xi$. We control both terms of

$$
\begin{aligned}
\Psi & =\left(\frac{\mathrm{d} y}{\mathrm{~d} \xi}\right)^{2} W_{2}+\left(\frac{\mathrm{d} y}{\mathrm{~d} \xi}\right)^{\frac{1}{2}} \frac{\mathrm{d}^{2}}{\mathrm{~d} \xi^{2}}\left(\frac{\mathrm{d} y}{\mathrm{~d} \xi}\right)^{-\frac{1}{2}} \\
& =\left(\frac{\mathrm{d} y}{\mathrm{~d} \xi}\right)^{2} W_{2}+\left(\frac{\mathrm{d} y}{\mathrm{~d} \xi}\right)^{-2}\left(-\frac{1}{2} \frac{\mathrm{d} y}{\mathrm{~d} \xi} \frac{\mathrm{d}^{3} y}{\mathrm{~d} \xi^{3}}+\frac{3}{4}\left(\frac{\mathrm{d}^{2} y}{\mathrm{~d} \xi^{2}}\right)^{2}\right)
\end{aligned}
$$

independently. For large $\xi$, we estimate the first term as

$$
\left|\left(\frac{\mathrm{d} y}{\mathrm{~d} \xi}\right)^{2} W_{2}\right| \leq\left|W_{2}\right| \frac{\xi^{2}-\alpha^{2}}{\left|W_{1}\right|} \lesssim \xi^{2}\left|W_{2}\right|
$$

in view of

$$
W_{1} \geq \frac{\Xi^{2}}{2}
$$

for $\xi$ sufficiently large. Further, for $\xi$ sufficiently large we have $\left|W_{2}\right| \lesssim e^{-2 \Xi y}$ and thus,

$$
\xi^{2}\left|W_{2}\right| \lesssim \xi^{2} e^{-2 \Xi y(\xi)} \lesssim e^{-\xi}
$$

in view of Lemma 3.4.1.

For the second term of (3.96), we use Lemma 3.4.1 to estimate

$$
\left|\left(\frac{\mathrm{d} y}{\mathrm{~d} \xi}\right)^{-2}\left(-\frac{1}{2} \frac{\mathrm{d} y}{\mathrm{~d} \xi} \frac{\mathrm{d}^{3} y}{\mathrm{~d} \xi^{3}}+\frac{3}{4}\left(\frac{\mathrm{d}^{2} y}{\mathrm{~d} \xi^{2}}\right)^{2}\right)\right| \lesssim \xi^{-2}
$$

for $\xi$ sufficiently large. Hence,

$$
|\Psi| \lesssim(1+\xi)^{-2}
$$

for $\xi$ sufficiently large. Recall that $\Psi$ is continuous everywhere and $\Omega=|x|^{\frac{1}{3}}$ such that

$$
\mathcal{V}_{0, \infty}\left(F_{1}\right) \lesssim \int_{0}^{\infty} \frac{|\Psi|}{\xi^{\frac{1}{3}} m^{\frac{1}{6}}} \mathrm{~d} \xi \lesssim m^{-\frac{1}{6}}
$$


Having controlled the error terms we now proceed to the definition of our fundamental solutions based on appropriate parabolic cylinder functions. We will apply [93, Theorem 1] which we recall for convenience of the reader in the following.

Proposition 3.4 ([93, Theorem 1]) Assume that for each value of m, the function $\Psi(m, \alpha, \xi)$ as defined in (3.84) is continuous in the region $\alpha \in[0, A], \xi \in$ $[0, \infty)$ and $\mathcal{V}_{0, \infty}\left(F_{1}\right)$ converges uniformly with respect to $\alpha$, where $F_{1}$ is as in (3.85). Then, the o.d.e. (3.83) has solutions $w_{1}(m, \alpha, \xi)$ and $w_{2}(m, \alpha, \xi)$ which are continuous, have continuous first and second partial $\xi$-derivatives and are given by

$$
\begin{aligned}
& w_{1}(m, \alpha, \xi)=U\left(-\frac{1}{2} m \alpha^{2}, \xi \sqrt{2 m}\right)+\epsilon_{1}(m, \alpha, \xi), \\
& w_{2}(m, \alpha, \xi)=\bar{U}\left(-\frac{1}{2} m \alpha^{2}, \xi \sqrt{2 m}\right)+\epsilon_{2}(m, \alpha, \xi),
\end{aligned}
$$

where $U$ and $\bar{U}$ are parabolic cylinder functions defined in Definition A.2 in the appendix. The error terms satisfy

$$
\begin{aligned}
& \frac{\left|\epsilon_{1}(m, \alpha, \xi)\right|}{M_{U}\left(-\frac{1}{2} m \alpha^{2}, \xi \sqrt{2 m}\right)}, \frac{\partial_{\xi} \epsilon_{1}(m, \alpha, \xi)}{\sqrt{2 m} N_{U}\left(-\frac{1}{2} m \alpha^{2}, \xi \sqrt{2 m}\right)} \\
& \leq E_{U}^{-1}\left(-\frac{1}{2} m \alpha^{2}, \xi \sqrt{2 m}\right)\left[e^{\frac{1}{2} \pi^{\frac{1}{2}} m^{-\frac{1}{2}} l_{1}\left(-\frac{1}{2} m \alpha^{2}\right) \mathcal{V}_{\xi, \infty}\left(F_{1}\right)}-1\right] \\
& \frac{\left|\epsilon_{2}(m, \alpha, \xi)\right|}{M_{U}\left(-\frac{1}{2} m \alpha^{2}, \xi \sqrt{2 m}\right)}, \frac{\partial_{\xi} \epsilon_{2}(m, \alpha, \xi)}{\sqrt{2 m} N_{U}\left(-\frac{1}{2} m \alpha^{2}, \xi \sqrt{2 m}\right)} \\
& \leq E_{U}\left(-\frac{1}{2} m \alpha^{2}, \xi \sqrt{2 m}\right)\left[e^{\frac{1}{2} \pi^{\frac{1}{2}} m^{-\frac{1}{2}} l_{1}\left(-\frac{1}{2} m \alpha^{2}\right) \mathcal{V}_{0, \xi}\left(F_{1}\right)}-1\right]
\end{aligned}
$$

and

$$
l_{1}(b)=\sup _{x \in(0, \infty)}\left(\Omega(x) \frac{M_{U}^{2}(b, x)}{\Gamma\left(\frac{1}{2}-b\right)}\right), b \leq 0 .
$$

For the definitions of $M_{U}, N_{U}, E_{U}$ refer to Appendix A.2. We will now apply the previous proposition with our estimate at hand.

Proposition 3.5 There exist solutions $w_{1}$ and $w_{2}$ of (3.83) satisfying

$$
w_{1}=U\left(-\frac{1}{2} m \alpha^{2}, \xi \sqrt{2 m}\right)+\tilde{\eta}_{1}
$$




$$
w_{2}=\bar{U}\left(-\frac{1}{2} m \alpha^{2}, \xi \sqrt{2 m}\right)+\tilde{\eta}_{2}
$$

The error terms satisfy

$$
\begin{gathered}
\tilde{\eta}_{1}=E_{U}^{-1}\left(-\frac{1}{2} m \alpha^{2}, \xi \sqrt{2 m}\right) M_{U}\left(-\frac{1}{2} m \alpha^{2}, \xi \sqrt{2 m}\right) O\left(m^{-\frac{2}{3}}\right) \\
\tilde{\eta}_{2}=E_{U}\left(-\frac{1}{2} m \alpha^{2}, \xi \sqrt{2 m}\right) M_{U}\left(-\frac{1}{2} m \alpha^{2}, \xi \sqrt{2 m}\right) O\left(m^{-\frac{2}{3}}\right) \\
\partial_{\xi} \tilde{\eta}_{1}=E_{U}^{-1}\left(-\frac{1}{2} m \alpha^{2}, \xi \sqrt{2 m}\right) N_{U}\left(-\frac{1}{2} m \alpha^{2}, \xi \sqrt{2 m}\right) O\left(m^{-\frac{1}{6}}\right) \\
\partial_{\xi} \tilde{\eta}_{2}=E_{U}\left(-\frac{1}{2} m \alpha^{2}, \xi \sqrt{2 m}\right) N_{U}\left(-\frac{1}{2} m \alpha^{2}, \xi \sqrt{2 m}\right) O\left(m^{-\frac{1}{6}}\right)
\end{gathered}
$$

uniformly in $\tilde{\lambda} \in\left[\Xi^{2}, \Xi^{2}+1\right]$ and $\xi \in[0, \infty)$. Moreover, $\tilde{\eta}_{2}(\xi=0)=$ $\partial_{\xi} \tilde{\eta}_{2}(\xi=0)=0$ and $\lim _{\xi \rightarrow \infty} \tilde{\eta}_{1}(\xi)=\lim _{\xi \rightarrow \infty} \partial_{\xi} \tilde{\eta}_{1}(\xi)=0$.

Proof We have chosen $\Omega(x)=|x|^{\frac{1}{3}}$ in (3.85). For this choice of $\Omega$, the quantity $l_{1}$ as defined in (3.107) satisfies $l_{1}(b) \lesssim 1$ uniformly in $b \leq 0$ which follows from Proposition A.1 and Definition A.5, see also [93, equation (6.15)]. Now, we recall that $\Psi$ is continuous for $(\xi, \alpha) \in[0, \infty) \times[0, A]$ and from Lemma 3.8 we have $\mathcal{V}_{\xi, \infty}\left(F_{1}\right), \mathcal{V}_{0, \xi}\left(F_{1}\right) \leq \mathcal{V}_{0, \infty}\left(F_{1}\right) \lesssim \frac{1}{m^{\frac{1}{6}}}$. Hence, we apply Proposition 3.4 and moreover estimate the error terms as

$$
\left|e^{\frac{1}{2} \pi^{\frac{1}{2}} m^{-\frac{1}{2}} l_{1}\left(-\frac{1}{2} m \alpha^{2}\right) \mathcal{V}_{\xi, \infty}\left(F_{1}\right)}-1\right|,\left|e^{\frac{1}{2} \pi^{\frac{1}{2}} m^{-\frac{1}{2}} l_{1}\left(-\frac{1}{2} m \alpha^{2}\right) \mathcal{V}_{0, \xi}\left(F_{1}\right)}-1\right| \lesssim m^{-\frac{2}{3}}
$$

from which the error bounds follow.

Remark 3.1 As $x \rightarrow \infty$, the function $U$ is recessive (decaying), whereas $\bar{U}$ is dominant (growing). Hence, $w_{1}$ is recessive and $w_{2}$ is dominant. We refer to $[94$, Chapter $5, \S 7.2]$ for further details.

Lemma 3.9 The Wronskian $\mathfrak{W}\left(w_{1}, w_{2}\right)$ satisfies

$$
\left|\mathfrak{W}\left(w_{1}, w_{2}\right)\right| \sim \sqrt{m} \Gamma\left(\frac{1}{2}+\frac{1}{2} m \alpha^{2}\right)
$$

for $m$ sufficiently large. 
Proof Since the Wronskian is independent of $\xi$, we compute it at $\xi=0$ such that $\tilde{\eta}_{2}=\partial_{\xi} \tilde{\eta}_{2}=0$. We obtain

$$
\begin{aligned}
\mathfrak{W}\left(w_{1}, w_{2}\right) & =\mathfrak{W}\left(U\left(-\frac{1}{2} m \alpha^{2}, \xi \sqrt{2 m}\right)+\tilde{\eta}_{1}, \bar{U}\left(-\frac{1}{2} m \alpha^{2}, \xi \sqrt{2 m}\right)\right) \\
& =\mathfrak{W}\left(U\left(-\frac{1}{2} m \alpha^{2}, \xi \sqrt{2 m}\right), \bar{U}\left(-\frac{1}{2} m \alpha^{2}, \xi \sqrt{2 m}\right)\right) \\
& +\mathfrak{W}\left(\tilde{\eta}_{1}, \bar{U}\left(-\frac{1}{2} m \alpha^{2}, \xi \sqrt{2 m}\right)\right),
\end{aligned}
$$

where each Wronskian is evaluated at $\xi=0$. We begin by computing (3.116). For $U(b, x)$ and $\bar{U}(b, x)$ we have the Wronskian identity $\mathfrak{W}(U, \bar{U})=$ $\sqrt{\frac{2}{\pi}} \Gamma\left(\frac{1}{2}-b\right)$, see [93, Equation (5.8)]. Thus, the chain rule yields

$$
\begin{aligned}
& \mathfrak{W}\left(U\left(-\frac{1}{2} m \alpha^{2}, \xi \sqrt{2 m}\right), \bar{U}\left(-\frac{1}{2} m \alpha^{2}, \xi \sqrt{2 m}\right)\right) \\
& =\sqrt{2 m} \sqrt{\frac{2}{\pi}} \Gamma\left(\frac{1}{2}+\frac{1}{2} m \alpha^{2}\right) .
\end{aligned}
$$

Now, we use (3.110), (3.112), Definition A.4 and (A.21)-(A.24) to estimate

$$
\begin{aligned}
& \left|\mathfrak{W}\left(\tilde{\eta}_{1}, \bar{U}\left(-\frac{1}{2} m \alpha^{2}, \xi \sqrt{2 m}\right)\right)(\xi=0)\right| \\
& \lesssim \sqrt{m}\left|\tilde{\eta}_{1}(\xi=0) \bar{U}^{\prime}\left(-\frac{1}{2} m \alpha^{2}, 0\right)\right|+\left|\eta_{1}^{\prime}(\xi=0) \bar{U}\left(-\frac{1}{2} m \alpha^{2}, 0\right)\right| \\
& \lesssim m^{-\frac{1}{6}}\left|M_{U}\left(-\frac{1}{2} m \alpha^{2}, 0\right) \bar{U}^{\prime}\left(-\frac{1}{2} m \alpha^{2}, 0\right)\right| \\
& \quad+m^{-\frac{1}{6}}\left|N_{U}\left(-\frac{1}{2} m \alpha^{2}, 0\right) \bar{U}\left(-\frac{1}{2} m \alpha^{2}, 0\right)\right| \\
& \lesssim m^{-\frac{1}{6}}\left(\left|\sqrt{U^{2}+\bar{U}^{2}}\left(-\frac{1}{2} m \alpha^{2}, 0\right) \bar{U}^{\prime}\left(-\frac{1}{2} m \alpha^{2}, 0\right)\right|\right. \\
& \left.\quad+\left|\sqrt{U^{\prime 2}+\left(\bar{U}^{\prime}\right)^{2}}\left(-\frac{1}{2} m \alpha^{2}, 0\right) \bar{U}\left(-\frac{1}{2} m \alpha^{2}, 0\right)\right|\right) \\
& \lesssim m^{-\frac{1}{6} 2^{\frac{1}{2}} m \alpha^{2}} \Gamma\left(\frac{1}{4}+\frac{1}{4} m \alpha^{2}\right) \Gamma\left(\frac{3}{4}+\frac{1}{4} m \alpha^{2}\right)
\end{aligned}
$$




$$
\begin{aligned}
& =m^{-\frac{1}{6}} 2^{\frac{1}{2} m \alpha^{2}} \Gamma\left(\frac{1}{2}+\frac{1}{2} m \alpha^{2}\right) 2^{1-2\left(\frac{1}{4}+\frac{1}{4} m \alpha^{2}\right)} \sqrt{\pi} \\
& \lesssim m^{-\frac{1}{6}} \Gamma\left(\frac{1}{2}+\frac{1}{2} m \alpha^{2}\right),
\end{aligned}
$$

where we also used the Legendre duplication formula $\Gamma(x) \Gamma\left(x+\frac{1}{2}\right)=$ $2^{1-2 x} \sqrt{\pi} \Gamma(2 x)$. This concludes the proof.

Lemma 3.10 The function $\sigma_{1}$ defined in (3.82) has the form

$$
\sigma_{1}=A_{1} w_{1}
$$

where $w_{1}$ is as in Proposition 3.5 and $A_{1} \neq 0$ is a real constant.

Proof Both functions $\sigma_{1}$ and $w_{1}$ are non-trivial solutions to (3.83) which are recessive as $\xi \rightarrow \infty(y \rightarrow \infty)$. The claim follows now as the space of solutions of (3.83) which are recessive as $\xi \rightarrow \infty$ is one-dimensional (see e.g. [94, Chapter 5, §7.2]).

Using the parabolic cylinder functions, we now define a solution $\sigma_{2}$ which is linearly independent of $\sigma_{1}$.

Definition 3.1 We define the solution $\sigma_{2}$ of (3.83) as

$$
\sigma_{2}:=\frac{1}{A_{1} \mathfrak{W}\left(w_{1}, w_{2}\right)} w_{2}
$$

and the solution $s_{2}$ to $(3.65)$ as

$$
s_{2}(y):=\left(\frac{\mathrm{d} y}{\mathrm{~d} \xi}\right)^{\frac{1}{2}} \sigma_{2}(\xi(y))
$$

A direct computation shows

Lemma 3.11 We have

$$
\mathfrak{W}_{y}\left(s_{1}, s_{2}\right)=\mathfrak{W}_{\xi}\left(\sigma_{1}, \sigma_{2}\right)=1
$$

Here, $\mathfrak{W}_{y}$ and $\mathfrak{W}_{\xi}$ denote the Wronskians with respect to the $y$ and $\xi$ variable.

Lemma 3.12 With $\sigma_{1}$ and $\sigma_{2}$ as defined in (3.82) and (3.121) we have

$$
\left|\sigma_{1}\right| \lesssim\left|A_{1}\right| E_{U}^{-1}\left(-\frac{1}{2} m \alpha^{2}, \xi \sqrt{2 m}\right) M_{U}\left(-\frac{1}{2} m \alpha^{2}, \xi \sqrt{2 m}\right)
$$




$$
\begin{aligned}
& \left|\sigma_{2}\right| \lesssim\left|\frac{1}{A_{1} \mathfrak{W}\left(w_{1}, w_{2}\right)}\right| E_{U}\left(-\frac{1}{2} m \alpha^{2}, \xi \sqrt{2 m}\right) M_{U}\left(-\frac{1}{2} m \alpha^{2}, \xi \sqrt{2 m}\right) \\
& \left|\sigma_{1}(\xi) \sigma_{2}(\xi)\right| \lesssim \frac{1}{\left|\mathfrak{W}\left(w_{1}, w_{2}\right)\right|} M_{U}^{2}\left(-\frac{1}{2} m \alpha^{2}, \xi \sqrt{2 m}\right) .
\end{aligned}
$$

Proof We estimate using (A.29) that

$$
\begin{aligned}
\left|\sigma_{1}\right| & =\left|A_{1} w_{1}\right|=\left|A_{1}\right|\left|U\left(-\frac{1}{2} m \alpha^{2}, \xi \sqrt{2 m}\right)+\tilde{\eta}_{1}\right| \\
& \lesssim\left|A_{1}\right| E_{U}^{-1}\left(-\frac{1}{2} m \alpha^{2}, \xi \sqrt{2 m}\right) M_{U}\left(-\frac{1}{2} m \alpha^{2}, \xi \sqrt{2 m}\right)
\end{aligned}
$$

and

$$
\begin{aligned}
\left|\sigma_{2}\right| & =\left|\frac{1}{A_{1} \mathfrak{W}\left(w_{1}, w_{2}\right)}\right|\left|w_{2}\right| \leq\left|\frac{1}{A_{1} \mathfrak{W}\left(w_{1}, w_{2}\right)}\right|\left|\bar{U}\left(-\frac{1}{2} m \alpha^{2}, \xi \sqrt{2 m}\right)+\tilde{\eta}_{2}\right| \\
& \lesssim\left|\frac{1}{A_{1} \mathfrak{W}\left(w_{1}, w_{2}\right)}\right| E_{U}\left(-\frac{1}{2} m \alpha^{2}, \xi \sqrt{2 m}\right) M_{U}\left(-\frac{1}{2} m \alpha^{2}, \xi \sqrt{2 m}\right) .
\end{aligned}
$$

Now, we recall the definition of $g_{p}$ in (3.66) as

$$
g_{p}(y):=s_{2}(y) \int_{y}^{\infty} s_{1}^{2}(\tilde{y}) F(\tilde{y}) \mathrm{d} \tilde{y}+s_{1}(y) \int_{0}^{y} s_{1}(\tilde{y}) s_{2}(\tilde{y}) F(\tilde{y}) \mathrm{d} \tilde{y} .
$$

for $s_{1}$ as in (3.58) and where we take $s_{2}$ as in (3.122). Now, we are in the position to show the main lemma of Sect. 3.4.1.

Lemma 3.13 Let $\tilde{\lambda} \in\left(\Xi^{2}, \Xi^{2}+1\right]$ and let $s_{2}$ as in (3.122). Then, $g_{p}$ satisfies

$$
\int_{0}^{\infty} g_{p}(y)^{2}\left(1-x(y)^{2}\right) \Delta_{x} \mathrm{~d} y \lesssim m
$$

Proof We plug (3.129) into the left hand side of (3.130) and we will estimate both terms independently.

For the first term, we change variables from $y$ to $\xi$, use that $x \mapsto E_{U}(b, x)$ is non-decreasing, as well as Lemma 3.12 to estimate 


$$
\begin{aligned}
& \int_{0}^{\infty} s_{2}^{2}(y)\left(\int_{y}^{\infty} s_{1}^{2}(\tilde{y}) F(\tilde{y}) \mathrm{d} \tilde{y}\right)^{2}\left(1-x(y)^{2}\right) \Delta_{x} \mathrm{~d} y \\
& =\int_{0}^{\infty} \sigma_{2}^{2}(\xi)\left(\int_{\xi}^{\infty} \sigma_{1}^{2}(\tilde{\xi}) F(\tilde{\xi}) \mathrm{d} \tilde{\xi}\right)^{2}\left(1-x(\xi)^{2}\right) \Delta_{x}(\xi) \mathrm{d} \xi \\
& \quad \lesssim \int_{0}^{\infty}\left[\frac { | M _ { U } ( - \frac { 1 } { 2 } m \alpha ^ { 2 } , \xi \sqrt { 2 m } ) | ^ { 2 } } { | \mathfrak { W } ( w _ { 1 } , w _ { 2 } ) | ^ { 2 } } \left(\int_{\xi}^{\infty}\left|\sigma_{1}(\tilde{\xi}) F(\tilde{\xi})\right|\right.\right. \\
& \left.\left.\left|M_{U}\left(-\frac{1}{2} m \alpha^{2}, \tilde{\xi} \sqrt{2 m}\right)\right| \mathrm{d} \tilde{\xi}\right)^{2}\left(1-x(\xi)^{2}\right) \Delta_{x}(\xi)\right] \mathrm{d} \xi
\end{aligned}
$$

Now, we use the bounds on $M_{U}$ and $\mathfrak{W}\left(w_{1}, w_{2}\right)$ from Proposition A.1 and Lemma 3.9 to deduce

$$
\begin{aligned}
\int_{0}^{\infty} & s_{2}^{2}(y)\left(\int_{y}^{\infty} s_{1}^{2}(\tilde{y}) F(\tilde{y}) \mathrm{d} \tilde{y}\right)^{2}\left(1-x(y)^{2}\right) \Delta_{x} \mathrm{~d} y \\
& \lesssim \frac{1}{m} \int_{0}^{\infty}\left(1-x(\xi)^{2}\right) \Delta_{x}(\xi) \mathrm{d} \xi\left(\int_{0}^{\infty}\left|\sigma_{1}(\tilde{\xi})\right||F(\tilde{\xi})| \mathrm{d} \tilde{\xi}\right)^{2} \\
& \lesssim \frac{1}{m} \int_{0}^{\infty}\left|s_{1}\right|^{2}\left(1-x(y)^{2}\right) \Delta_{x}(y) \mathrm{d} y \int_{0}^{\infty} \frac{|F|^{2}}{\Delta_{x}\left(1-x(y)^{2}\right)} \frac{\mathrm{d} \xi}{\mathrm{d} y} \mathrm{~d} y \\
& \lesssim \frac{1}{m} \int_{0}^{\infty} \Delta_{x}\left(1-x^{2}\right)\left[\partial_{\xi} \lambda+2 \Xi\left(a m \omega_{-}+\frac{\epsilon}{m}\right) \frac{x^{2}}{\Delta_{x}}+2 m \frac{\Xi}{\Delta_{x}} \frac{a^{2}}{l^{2}} x^{2}\right]^{2} \frac{\mathrm{d} \xi}{\mathrm{d} y} \mathrm{~d} y \\
& \lesssim m
\end{aligned}
$$

where we used the Cauchy-Schwarz inequality and the fact that $s_{1}$ satisfies (3.59) as well as (3.44).

For the second term we argue similarly and obtain

$$
\begin{aligned}
& \left|\int_{0}^{\infty} s_{1}^{2}(y)\left(\int_{0}^{y} s_{2}(\tilde{y}) s_{1}(\tilde{y}) F(\tilde{y}) \mathrm{d} \tilde{y}\right)^{2}\left(1-x(y)^{2}\right) \Delta_{x} \mathrm{~d} y\right| \\
& \quad \lesssim \int_{0}^{\infty} s_{1}^{2}(y)\left(1-x(y)^{2}\right) \Delta_{x} \mathrm{~d} y\left(\int_{0}^{\infty} s_{2}(\tilde{y}) s_{1}(\tilde{y}) F(\tilde{y}) \mathrm{d} \tilde{y}\right)^{2} \\
& \quad \lesssim\left(\int_{0}^{\infty} \frac{M_{U}^{2}\left(-\frac{1}{2} m \alpha^{2}, \xi \sqrt{2 m}\right)}{\left|\mathfrak{W}\left(w_{1}, w_{2}\right)\right|}|F(\xi)| \mathrm{d} \xi\right)^{2} \\
& \quad \lesssim \frac{1}{m}\left(\int_{0}^{\infty} \Delta_{x}\left(1-x(y)^{2}\right)\left|\partial_{\xi} \lambda+2 \Xi\left(a m \omega_{-}+\frac{\epsilon}{m}\right) \frac{x^{2}}{\Delta_{x}}+2 m \frac{\Xi}{\Delta_{x}} \frac{a^{2}}{l^{2}} x^{2}\right| \frac{\mathrm{d} \xi}{\mathrm{d} y} \mathrm{~d} y\right)^{2} \\
& \quad \lesssim m .
\end{aligned}
$$




\subsubsection{The case $\tilde{\lambda} \in\left(\Xi^{2}+1, \infty\right)$}

For the parameter range $\tilde{\lambda} \in\left(\Xi^{2}+1, \infty\right)$ we consider $\lambda=m^{2} \tilde{\lambda}$ as a large parameter and re-write the o.d.e. (3.64) as

$$
-\frac{\mathrm{d}^{2}}{\mathrm{~d} y^{2}} s_{p}+m^{2} \tilde{\lambda} \tilde{W}_{1} s_{p}+W_{2} s_{p}=F s_{1}
$$

where

$$
\begin{aligned}
\tilde{W}_{1}= & \tilde{W}_{1}(y):=\frac{W_{1}}{\tilde{\lambda}}=\frac{\Xi^{2}}{\tilde{\lambda}}-\left[\Xi a^{2} \omega_{-}^{2}+2 a \omega_{-} \Xi \frac{a^{2}}{l^{2}}\right] \frac{x^{2}\left(1-x^{2}\right)}{\tilde{\lambda}} \\
& -\Delta_{x}\left(1-x^{2}\right) .
\end{aligned}
$$

We also recall the homogeneous o.d.e. (3.65)

$$
-\frac{\mathrm{d}^{2}}{\mathrm{~d} y^{2}} g+m^{2} \tilde{\lambda} \tilde{W}_{1} g+W_{2} g=0 .
$$

Recall also that $s_{1}$ as defined in (3.58) is a solution of (3.136). As before, we define $y_{0}$ as the unique non-negative root of $\tilde{W}_{1}(y)$. It satisfies

$$
y_{0} \sim \log (\tilde{\lambda})
$$

for sufficiently large $\tilde{\lambda}$, where we note that $y_{0}$ becomes arbitrarily large for $\tilde{\lambda} \rightarrow \infty$. Indeed, to show (3.137), we note that for large $\tilde{\lambda}$, from (3.135) we see that $x\left(y_{0}\right)$ satisfies

$$
\begin{aligned}
\Xi^{2}= & {\left[\Xi a^{2} \omega_{-}^{2}+2 a \omega_{-} \Xi \frac{a^{2}}{l^{2}}\right] x\left(y_{0}\right)^{2}\left(1-x\left(y_{0}\right)^{2}\right) } \\
& +\tilde{\lambda} \Delta_{x\left(y_{0}\right)}\left(1-x\left(y_{0}\right)^{2}\right) \sim \tilde{\lambda}\left(1-x\left(y_{0}\right)\right) .
\end{aligned}
$$

Then, (3.137) follows from $1-x \sim e^{-2 \Xi y}$ for $y$ sufficiently large (recall (3.57)). Our estimates will be uniform in the limit $\tilde{\lambda} \rightarrow \infty$.

Lemma 3.14 In the region $0 \leq y \leq y_{0}-1$ we have

$$
\frac{1}{\tilde{\lambda}} \lesssim-\tilde{W}_{1} \lesssim 1, \quad \frac{\mathrm{d} \tilde{W}_{1}}{\mathrm{~d} y} \lesssim\left|\tilde{W}_{1}\right|, \quad\left|\frac{\mathrm{d}^{2} \tilde{W}_{1}}{\mathrm{~d} y^{2}}\right| \lesssim \frac{\mathrm{d} \tilde{W}_{1}}{\mathrm{~d} y}+\left(1-x(y)^{2}\right)\left|\tilde{W}_{1}\right|
$$


For $y_{0}-1 \leq y \leq y_{0}+1$, we have

$$
\frac{\left|y-y_{0}\right|}{\tilde{\lambda}} \lesssim\left|\tilde{W}_{1}\right| \lesssim \frac{1}{\tilde{\lambda}}, \quad \frac{\mathrm{d} \tilde{W}_{1}}{\mathrm{~d} y} \sim \frac{1}{\tilde{\lambda}}, \quad\left|\frac{\mathrm{d}^{2} \tilde{W}_{1}}{\mathrm{~d} y^{2}}\right| \lesssim \frac{1}{\tilde{\lambda}}, \quad\left|\frac{\mathrm{d}^{3} \tilde{W}_{1}}{\mathrm{~d} y^{3}}\right| \lesssim \frac{1}{\tilde{\lambda}}, \quad\left|\frac{\mathrm{d}^{4} \tilde{W}_{1}}{\mathrm{~d} y^{4}}\right| \lesssim \frac{1}{\tilde{\lambda}} .
$$

For $y_{0}+1 \leq y<\infty$, we have

$$
\tilde{W}_{1} \sim \frac{1}{\tilde{\lambda}} \quad \text { and } \quad \frac{\mathrm{d} \tilde{W}_{1}}{\mathrm{~d} y},\left|\frac{\mathrm{d}^{2} \tilde{W}_{1}}{\mathrm{~d} y^{2}}\right| \lesssim \frac{\mathrm{d} x}{\mathrm{~d} y} \lesssim \frac{1}{\tilde{\lambda}} .
$$

Proof From Lmma 3.1 we have that $\tilde{W}_{1}$ is increasing on $y \in[0, \infty)$ and moreover, for $y \in\left[y_{0}-1, y_{0}+1\right]$ we have that

$$
\frac{\mathrm{d} \tilde{W}_{1}}{\mathrm{~d} y}=\frac{\mathrm{d} \tilde{W}_{1}}{\mathrm{~d} x} \frac{\mathrm{d} x}{\mathrm{~d} y} \gtrsim x(y) \frac{\mathrm{d} x}{\mathrm{~d} y} \gtrsim \frac{1}{\tilde{\lambda}} .
$$

Thus, for $0 \leq y \leq y_{0}-1$,

$$
-\tilde{W}_{1}(y) \geq-\tilde{W}_{1}\left(y_{0}-1\right) \geq \int_{y_{0}-1}^{y_{0}} \frac{\mathrm{d} \tilde{W}_{1}}{\mathrm{~d} y} \mathrm{~d} \tilde{y} \gtrsim \frac{1}{\tilde{\lambda}} .
$$

Moreover, for $0 \leq y \leq y_{0}-1$,

$$
\frac{\mathrm{d} \tilde{W}_{1}}{\mathrm{~d} y} \lesssim \frac{\mathrm{d} x}{\mathrm{~d} y}=\Delta_{x}\left(1-x^{2}\right) \lesssim\left|\tilde{W}_{1}\right|+\frac{1}{\tilde{\lambda}} \lesssim\left|\tilde{W}_{1}\right|
$$

using the definition of $\tilde{W}_{1}$ and $\left|\tilde{W}_{1}\right| \gtrsim \frac{1}{\tilde{\lambda}}$. Similarly, we obtain

$$
\left|\frac{\mathrm{d}^{2} \tilde{W}_{1}}{\mathrm{~d} y^{2}}\right| \lesssim\left(1-x(y)^{2}\right) \frac{\mathrm{d} \tilde{W}_{1}}{\mathrm{~d} x}+\left(1-x(y)^{2}\right)^{2}\left|\frac{\mathrm{d}^{2} \tilde{W}_{1}}{\mathrm{~d} x^{2}}\right| \lesssim \frac{\mathrm{d} \tilde{W}_{1}}{\mathrm{~d} y}+\left(1-x(y)^{2}\right)\left|\tilde{W}_{1}\right| .
$$

In the region $y \in\left[y_{0}-1, y_{0}+1\right]$, recall from (3.142) that $\frac{\mathrm{d} \tilde{W}_{1}}{\mathrm{~d} y} \gtrsim \frac{1}{\tilde{\lambda}}$. Moreover, just as in (3.145), we obtain

$$
\left|\frac{\mathrm{d} \tilde{W}_{1}}{\mathrm{~d} y}\right|,\left|\frac{\mathrm{d}^{2} \tilde{W}_{1}}{\mathrm{~d} y^{2}}\right|,\left|\frac{\mathrm{d}^{3} \tilde{W}_{1}}{\mathrm{~d} y^{3}}\right|,\left|\frac{\mathrm{d}^{4} \tilde{W}_{1}}{\mathrm{~d} y^{4}}\right| \lesssim \frac{\mathrm{d} x}{\mathrm{~d} y} \lesssim \frac{1}{\tilde{\lambda}} .
$$

In the region $y \in\left(y_{0}+1,+\infty\right)$, analogous to (3.143), we have

$$
\frac{1}{\tilde{\lambda}} \lesssim \tilde{W}_{1} \lesssim \frac{1}{\tilde{\lambda}}
$$


and moreover,

$$
\frac{\mathrm{d} \tilde{W}_{1}}{\mathrm{~d} y},\left|\frac{\mathrm{d}^{2} \tilde{W}_{1}}{\mathrm{~d} y^{2}}\right| \lesssim \frac{\mathrm{d} x}{\mathrm{~d} y} \lesssim \frac{1}{\tilde{\lambda}}
$$

With the estimates of Lemma 3.14 in hand we will define the variable $\varsigma$ as

$$
\frac{2}{3} \varsigma^{\frac{3}{2}}=\int_{y_{0}}^{y} \sqrt{\tilde{W}_{1}(y)} \mathrm{d} y
$$

for $y \geq y_{0}$ and

$$
\frac{2}{3}(-\varsigma)^{\frac{3}{2}}=\int_{y}^{y_{0}} \sqrt{-\tilde{W}_{1}(y)} \mathrm{d} y
$$

for $y \leq y_{0}$. We denote

$$
\varsigma_{0}:=\varsigma(y=0)=-\left(\frac{3}{2} \int_{0}^{y_{0}} \sqrt{-\tilde{W}_{1}(y)} \mathrm{d} y\right)^{\frac{2}{3}} .
$$

We further introduce the error control function

$$
H(y):=\int_{y_{0}}^{y} \frac{1}{\left|\tilde{W}_{1}\right|^{\frac{1}{4}}} \frac{\mathrm{d}^{2}}{\mathrm{~d} y^{2}}\left(\left|\tilde{W}_{1}\right|^{-\frac{1}{4}}\right)-\frac{W_{2}}{\left|\tilde{W}_{1}\right|^{\frac{1}{2}}}-\frac{5\left|\tilde{W}_{1}\right|^{\frac{1}{2}}}{16 \varsigma(y)^{3}} \mathrm{~d} y
$$

The fact that $H$ is absolutely continuous is a standard result and follows from [92, Lemma, Sect. 4], see also [94, Lemma 3.1, Chapter 11]. In the following we establish a quantitative version of this.

Lemma 3.15 The error control function $H$ defined in (3.152) satisfies

$$
\mathcal{V}_{0, \infty}(H) \lesssim \tilde{\lambda}^{\frac{1}{2}}
$$

Proof Since $H$ is absolutely continuous we compute

$$
\begin{aligned}
\mathcal{V}_{0, \infty}(H)= & \int_{0}^{y_{0}-1}\left|\frac{1}{\left|\tilde{W}_{1}\right|^{\frac{1}{4}}} \frac{\mathrm{d}^{2}}{\mathrm{~d} y^{2}}\left(\left|\tilde{W}_{1}\right|^{-\frac{1}{4}}\right)-\frac{W_{2}}{\left|\tilde{W}_{1}\right|^{\frac{1}{2}}}-\frac{5\left|\tilde{W}_{1}\right|^{\frac{1}{2}}}{16 \varsigma(y)^{3}}\right| \mathrm{d} y \\
& +\int_{y_{0}-1}^{y_{0}+1}\left|\frac{1}{\left|\tilde{W}_{1}\right|^{\frac{1}{4}}} \frac{\mathrm{d}^{2}}{\mathrm{~d} y^{2}}\left(\left|\tilde{W}_{1}\right|^{-\frac{1}{4}}\right)-\frac{W_{2}}{\left|\tilde{W}_{1}\right|^{\frac{1}{2}}}-\frac{5\left|\tilde{W}_{1}\right|^{\frac{1}{2}}}{16 \varsigma(y)^{3}}\right| \mathrm{d} y
\end{aligned}
$$




$$
\begin{aligned}
& +\int_{y_{0}+1}^{+\infty}\left|\frac{1}{\left|\tilde{W}_{1}\right|^{\frac{1}{4}}} \frac{\mathrm{d}^{2}}{\mathrm{~d} y^{2}}\left(\left|\tilde{W}_{1}\right|^{-\frac{1}{4}}\right)-\frac{W_{2}}{\left|\tilde{W}_{1}\right|^{\frac{1}{2}}}-\frac{5\left|\tilde{W}_{1}\right|^{\frac{1}{2}}}{16 \varsigma(y)^{3}}\right| \mathrm{d} y \\
= & : I+I I+I I I
\end{aligned}
$$

and estimate each term independently relying on Lemma 3.14.

Term I We estimate term $I$ as

$$
I \lesssim \int_{0}^{y_{0}-1} \frac{1}{\left|\tilde{W}_{1}\right|^{\frac{5}{2}}}\left(\frac{\mathrm{d} \tilde{W}_{1}}{\mathrm{~d} y}\right)^{2}+\frac{1}{\left|\tilde{W}_{1}\right|^{\frac{3}{2}}}\left|\frac{\mathrm{d}^{2} \tilde{W}_{1}}{\mathrm{~d} y^{2}}\right|+\frac{\left|W_{2}\right|}{\left|\tilde{W}_{1}\right|^{\frac{1}{2}}}+\frac{\left|\tilde{W}_{1}\right|^{\frac{1}{2}}}{\varsigma^{3}} \mathrm{~d} y .
$$

We consider the first term appearing in (3.155) and in view of Lemma 3.14 we obtain

$$
\left.\int_{0}^{y_{0}-1} \frac{1}{\left|\tilde{W}_{1}\right|^{\frac{5}{2}}}\left(\frac{\mathrm{d} \tilde{W}_{1}}{\mathrm{~d} y}\right)^{2} \mathrm{~d} y \lesssim \int_{0}^{y_{0}-1} \frac{\frac{\mathrm{d} \tilde{W}_{1}}{\mathrm{~d} y}}{\left|\tilde{W}_{1}\right|^{\frac{3}{2}}} \mathrm{~d} y \lesssim \frac{1}{\left|\tilde{W}_{1}\right|^{\frac{1}{2}}}\right|_{0} ^{y_{0}-1} \lesssim \tilde{\lambda}^{\frac{1}{2}}
$$

For the second term involving the second derivative, we use (3.139) to conclude that

$$
\int_{0}^{y_{0}-1} \frac{1}{\left|\tilde{W}_{1}\right|^{\frac{3}{2}}}\left|\frac{\mathrm{d}^{2} \tilde{W}_{1}}{\mathrm{~d} y^{2}}\right| \mathrm{d} y \lesssim \int_{0}^{y_{0}-1} \frac{\frac{\mathrm{d} \tilde{W}_{1}}{\mathrm{~d} y}}{\left|\tilde{W}_{1}\right|^{\frac{3}{2}}}+\frac{1-x(y)^{2}}{\left|\tilde{W}_{1}\right|^{\frac{1}{2}}} \mathrm{~d} y \lesssim \tilde{\lambda}^{\frac{1}{2}} .
$$

For the third term we use that $\left|W_{2}\right| \lesssim 1-x(y)^{2}$ such that

$$
\int_{0}^{y_{0}-1} \frac{\left|W_{2}\right|}{\left|\tilde{W}_{1}\right|^{\frac{1}{2}}} \lesssim \tilde{\lambda}^{\frac{1}{2}} \int_{0}^{\infty}\left(1-x(y)^{2}\right) \mathrm{d} y \lesssim \tilde{\lambda}^{\frac{1}{2}}
$$

For the last term in (3.155), we have

$$
\begin{aligned}
\int_{0}^{y_{0}-1} \frac{\left|\tilde{W}_{1}\right|^{\frac{1}{2}}}{\varsigma^{3}} \mathrm{~d} y & \lesssim \int_{0}^{y_{0}-1} \frac{\sqrt{-\tilde{W}_{1}}}{\left(\int_{y}^{y_{0}} \sqrt{-\tilde{W}_{1}} \mathrm{~d} \tilde{y}\right)^{2}} \mathrm{~d} y \lesssim \frac{1}{\int_{y_{0}-1}^{y_{0}} \sqrt{-\tilde{W}_{1}} \mathrm{~d} \tilde{y}} \\
& \lesssim \frac{1}{\int_{y_{0}-1}^{y_{0}} \sqrt{\left|\tilde{y}-y_{0}\right| \tilde{\lambda}^{-1}} \mathrm{~d} \tilde{y}} \lesssim \tilde{\lambda}^{\frac{1}{2}}
\end{aligned}
$$

Term II For this term, we use Taylor's theorem around the point $y=y_{0}$. We will now only consider the region $y \in\left[y_{0}-1, y_{0}+1\right]$ and use the 
notiation ' $=\frac{\mathrm{d}}{\mathrm{d} y}$ throughout the following paragraph. We write $\tilde{W}_{1}(y)=$ $\left(y-y_{0}\right) \tilde{W}_{1}^{\prime}\left(y_{0}\right)+\frac{1}{2}\left(y-y_{0}\right)^{2} \tilde{W}_{1}^{\prime \prime}\left(y_{0}\right)+\frac{1}{6}\left(y-y_{0}\right)^{3} \tilde{W}_{1}^{\prime \prime \prime}\left(y_{0}\right)+\frac{1}{24}\left(y-y_{0}\right)^{4} R_{W_{1}}(y)$, where the remainder $R_{W_{1}}(y)$ is smooth and satisfies $R_{W_{1}}(y)=\tilde{W}_{1}^{\prime \prime \prime \prime}(\xi)$ for some $\xi$ between $y_{0}$ and $y$. Thus,

$$
\begin{aligned}
\tilde{W}_{1}(y)= & \left(y-y_{0}\right) \tilde{W}_{1}^{\prime}\left(y_{0}\right)+\frac{1}{2}\left(y-y_{0}\right)^{2} \tilde{W}_{1}^{\prime \prime}\left(y_{0}\right) \\
& +\frac{1}{6}\left(y-y_{0}\right)^{3} \tilde{W}_{1}^{\prime \prime \prime}\left(y_{0}\right)+O\left(\tilde{\lambda}^{-1}\left(y-y_{0}\right)^{4}\right)
\end{aligned}
$$

in view of (3.140). We note that $\zeta^{3}=\frac{9}{4}\left(\int_{y_{0}}^{y} \sqrt{\left|\tilde{W}_{1}(\tilde{y})\right|} \mathrm{d} \tilde{y}\right)^{2}$ which we expand as

$$
\begin{aligned}
\zeta^{3}(y)= & \tilde{W}^{\prime}\left(y_{0}\right)\left(y-y_{0}\right)^{3}+\frac{3}{10} \tilde{W}_{1}^{\prime \prime}\left(y_{0}\right)\left(y-y_{0}\right)^{4} \\
& +\frac{1}{700}\left(-\frac{3 \tilde{W}_{1}^{\prime \prime}\left(y_{0}\right)^{2}}{W^{\prime}\left(y_{0}\right)}+50 \tilde{W}_{1}^{\prime \prime \prime}\left(y_{0}\right)\right)\left(y-y_{0}\right)^{5}+\tilde{\lambda}^{-1} O\left(\left|y-y_{0}\right|^{6}\right) .
\end{aligned}
$$

Then, we further expand

$$
\begin{aligned}
-\frac{5}{16} \frac{\tilde{W}_{1}}{\xi^{3}}= & \frac{-5}{16\left|y-y_{0}\right|^{2}}-\frac{\tilde{W}_{1}^{\prime \prime}\left(y_{0}\right)}{16 W^{\prime}\left(y_{0}\right)\left(y-y_{0}\right)} \\
& +\frac{117 \tilde{W}_{1}^{\prime \prime}\left(y_{0}\right)^{2}-200 \tilde{W}_{1}^{\prime}\left(y_{0}\right) \tilde{W}_{1}^{\prime \prime \prime}\left(y_{0}\right)}{6720 \tilde{W}_{1}^{\prime}\left(y_{0}\right)^{2}}+O\left(\left|y-y_{0}\right|\right) .
\end{aligned}
$$

Here, the error in $O\left(\left|y-y_{0}\right|\right)$ is estimated using (3.140) and we note that the homogeneity of (3.162) is such that no powers of $\tilde{\lambda}$ occur.

We also expand $\left|\tilde{W}_{1}\right|^{\frac{1}{4}} \frac{\mathrm{d}^{2}}{\mathrm{~d} y^{2}}\left(\left|\tilde{W}_{1}\right|^{-\frac{1}{4}}\right)$ around $y=y_{0}$ and obtain

$$
\begin{aligned}
\left|\tilde{W}_{1}\right|^{\frac{1}{4}} \frac{\mathrm{d}^{2}}{\mathrm{~d} y^{2}}\left(\left|\tilde{W}_{1}\right|^{-\frac{1}{4}}\right)= & \frac{5}{16\left|y-y_{0}\right|^{2}}+\frac{\tilde{W}_{1}^{\prime \prime}\left(y_{0}\right)}{16 \tilde{W}_{1}^{\prime}\left(y_{0}\right)\left(y-y_{0}\right)} \\
& +\frac{9 \tilde{W}_{1}^{\prime \prime}\left(y_{0}\right)^{2}-8 \tilde{W}_{1}^{\prime}\left(y_{0}\right) \tilde{W}_{1}^{\prime \prime \prime}\left(y_{0}\right)}{192 \tilde{W}_{1}^{\prime}\left(y_{0}\right)^{2}}+O\left(\left|y-y_{0}\right|\right) .
\end{aligned}
$$


Thus,

$$
\begin{aligned}
& \left|\tilde{W}_{1}\right|^{\frac{1}{4}} \frac{\mathrm{d}^{2}}{\mathrm{~d} y^{2}}\left(\left|\tilde{W}_{1}\right|^{-\frac{1}{4}}\right)-\frac{5}{16} \frac{\tilde{W}_{1}}{\xi^{3}} \\
& =\frac{9 \tilde{W}_{1}^{\prime \prime}\left(y_{0}\right)-10 \tilde{W}_{1}^{\prime \prime \prime}\left(y_{0}\right) \tilde{W}_{1}^{\prime}\left(y_{0}\right)}{140 \tilde{W}_{1}^{\prime}\left(y_{0}\right)^{2}}+O\left(\left|y-y_{0}\right|\right)
\end{aligned}
$$

from we estimate (using Lemma 3.14)

$$
\begin{aligned}
& \left|\frac{1}{\left|\tilde{W}_{1}\right|^{\frac{1}{4}}} \frac{\mathrm{d}^{2}}{\mathrm{~d} y^{2}}\left(\left|\tilde{W}_{1}\right|^{-\frac{1}{4}}\right)-\frac{W_{2}}{\left|\tilde{W}_{1}\right|^{\frac{1}{2}}}-\frac{5\left|\tilde{W}_{1}\right|^{\frac{1}{2}}}{16 \varsigma(y)^{3}}\right| \\
& \quad \lesssim \frac{1}{\left|W_{1}\right|^{\frac{1}{2}}}\left|\frac{\left|9 \tilde{W}_{1}^{\prime \prime}\left(y_{0}\right)-10 \tilde{W}_{1}^{\prime \prime \prime}\left(y_{0}\right) \tilde{W}_{1}^{\prime}\left(y_{0}\right)\right|}{140 \tilde{W}_{1}^{\prime}\left(y_{0}\right)^{2}}+\right| W_{2}\left|+O\left(\left|y-y_{0}\right|\right)\right| \\
& \quad \lesssim \frac{1}{\left|W_{1}\right|^{\frac{1}{2}}} \lesssim \frac{\tilde{\lambda}^{\frac{1}{2}}}{\left|y-y_{0}\right|^{\frac{1}{2}}}
\end{aligned}
$$

uniformly in $y \in\left[y_{0}-1, y_{0}+1\right]$ from which we conclude that $|I I| \lesssim \tilde{\lambda}^{\frac{1}{2}}$.

Term II I In the region $y \in\left[y_{0}+1, \infty\right)$ we first have

$$
\varsigma^{3}(y) \gtrsim\left(\int_{y_{0}}^{y_{0}+1} \sqrt{\tilde{W}_{1}} \mathrm{~d} \tilde{y}\right)^{2}+\left(\int_{y_{0}+1}^{y} \sqrt{\tilde{W}_{1}} \mathrm{~d} \tilde{y}\right)^{2} \gtrsim \frac{1}{\tilde{\lambda}}+\left(y-y_{0}-1\right)^{2} \frac{1}{\tilde{\lambda}}
$$

such that

$$
\frac{\left|\tilde{W}_{1}\right|^{\frac{1}{2}}}{\varsigma^{3}} \lesssim \frac{\tilde{\lambda}^{\frac{1}{2}}}{1+\left(y-y_{0}-1\right)^{2}}
$$

which is integrable at $y=\infty$. Moreover, we have

$$
\left|\frac{1}{\left|\tilde{W}_{1}\right|^{\frac{1}{4}}} \frac{\mathrm{d}^{2}}{\mathrm{~d} y^{2}}\left(\left|\tilde{W}_{1}\right|^{-\frac{1}{4}}\right)-\frac{W_{2}}{\left|\tilde{W}_{1}\right|^{\frac{1}{2}}}\right| \lesssim \frac{\mathrm{d} x}{\mathrm{~d} y}\left(\frac{\frac{\mathrm{d} \tilde{W}_{1}}{\mathrm{~d} y}}{\tilde{W}_{1}^{\frac{3}{2}}}+\frac{1}{\tilde{W}_{1}^{\frac{1}{2}}}\right) \lesssim \frac{\mathrm{d} x}{\mathrm{~d} y} \tilde{\lambda}^{\frac{1}{2}}
$$

Combining the estimates (3.167) and (3.168) we obtain that $|I I I| \lesssim \tilde{\lambda}^{\frac{1}{2}}$ which concludes the proof. 
Finally, we also introduce

$$
\hat{W}_{1}:=\frac{\tilde{W}_{1}}{\varsigma} \text { or equivalently } \quad \hat{W}_{1}=\left(\frac{\mathrm{d} \varsigma}{\mathrm{d} y}\right)^{2} \text {, }
$$

which we will bound from below in the following. To do that we also use the error-control function $M_{\mathrm{Ai}}$ which is defined in (A.6) in the appendix.

Lemma 3.16 We have

$$
\frac{M_{\mathrm{Ai}}\left(\lambda^{\frac{1}{3}} \zeta(y)\right)}{\hat{W}_{1}^{\frac{1}{4}}} \lesssim \tilde{\lambda}^{\frac{1}{6}}
$$

Proof First, for $y_{0}-1 \leq y \leq y_{0}$ we have

$$
\frac{2}{3}(-\varsigma)^{\frac{3}{2}}=\int_{y}^{y_{0}} \sqrt{\left|\tilde{W}_{1}\right|} \mathrm{d} \tilde{y} \leq\left(y_{0}-y\right) \sqrt{-\tilde{W}_{1}(y)}
$$

and for $y_{0} \leq y \leq y_{0}+1$ we have

$$
\frac{2}{3} \varsigma^{\frac{3}{2}}=\int_{y_{0}}^{y} \sqrt{\tilde{W}_{1}} \mathrm{~d} \tilde{y} \leq\left(y-y_{0}\right) \sqrt{\tilde{W}_{1}(y)}
$$

where we have used the monotonicity of $\tilde{W}_{1}$. Hence,

$$
\hat{W}_{1}=\frac{\tilde{W}_{1}}{\varsigma} \gtrsim\left(\frac{\tilde{W}_{1}}{y-y_{0}}\right)^{\frac{2}{3}} \gtrsim \tilde{\lambda}^{-\frac{2}{3}}
$$

for $\left|y-y_{0}\right| \leq 1$. Now, using $M_{\mathrm{Ai}}(x) \lesssim 1$ we conclude that for $\left|y-y_{0}\right| \leq 1$ we have

$$
\frac{M_{\mathrm{Ai}}\left(\lambda^{\frac{1}{3}} \varsigma(y)\right)}{\hat{W}_{1}^{\frac{1}{4}}} \lesssim \frac{1}{\hat{W}_{1}^{\frac{1}{4}}} \lesssim \tilde{\lambda}^{\frac{1}{6}}
$$

For $\left|y-y_{0}\right| \geq 1$, we use that $M_{\mathrm{Ai}}(x) \lesssim|x|^{-\frac{1}{4}}$ to obtain

$$
\frac{M_{\mathrm{Ai}}\left(\lambda^{\frac{1}{3}} \varsigma(y)\right)}{\hat{W}_{1}^{\frac{1}{4}}} \lesssim \frac{|\zeta|^{\frac{1}{4}}}{|\zeta|^{\frac{1}{4}} \lambda^{\frac{1}{12}}\left|\tilde{W}_{1}\right|^{\frac{1}{4}}} \lesssim \frac{\tilde{\lambda}^{\frac{1}{4}}}{\lambda^{\frac{1}{12}}} \lesssim \tilde{\lambda}^{\frac{1}{6}} m^{-\frac{1}{6}} \lesssim \tilde{\lambda}^{\frac{1}{6}}
$$


Now, we are in the position to define the following fundamental solutions.

Proposition 3.6 There exist solutions $w_{1}$ and $w_{2}$ of (3.136) satisfying

$$
\begin{aligned}
& w_{1}=\frac{1}{\hat{W}_{1}^{\frac{1}{4}}}\left(\operatorname{Ai}\left(\lambda^{\frac{1}{3}} \varsigma(y)\right)+\eta_{\mathrm{Ai}}(\lambda, y)\right), \\
& w_{2}=\frac{1}{\hat{W}_{1}^{\frac{1}{4}}}\left(\operatorname{Bi}\left(\lambda^{\frac{1}{3}} \varsigma(y)\right)+\eta_{\mathrm{Bi}}(\lambda, y)\right),
\end{aligned}
$$

where

$$
\begin{aligned}
& \frac{\left|\eta_{\mathrm{Ai}}(\lambda, y)\right|}{M_{\mathrm{Ai}}\left(\lambda^{\frac{1}{3}} \varsigma\right)}, \frac{\left|\partial_{y} \eta_{\mathrm{Ai}}(\lambda, y)\right|}{\lambda^{\frac{1}{3}} N_{\mathrm{Ai}}\left(\lambda^{\frac{1}{3}} \varsigma\right) \hat{W}_{1}^{\frac{1}{2}}} \lesssim E_{\mathrm{Ai}}^{-1}\left(\lambda^{\frac{1}{3}} \varsigma\right) m^{-1}, \\
& \frac{\left|\eta_{\mathrm{Bi}}(\lambda, y)\right|}{M_{\mathrm{Ai}}\left(\lambda^{\frac{1}{3}} \varsigma\right)}, \frac{\left|\partial_{y} \eta_{\mathrm{Bi}}(\lambda, y)\right|}{\lambda^{\frac{1}{3}} N_{\mathrm{Ai}}\left(\lambda^{\frac{1}{3}} \varsigma\right) \hat{W}_{1}^{\frac{1}{2}}} \lesssim E_{\mathrm{Ai}}\left(\lambda^{\frac{1}{3}} \varsigma\right) m^{-1} .
\end{aligned}
$$

Moreover, the Wronskian of $w_{1}$ and $w_{2}$ satisfies

$$
\left|\mathfrak{W}\left(w_{1}, w_{2}\right)\right| \sim \lambda^{\frac{1}{3}}
$$

Proof This follows from [94, Chapter 11, Theorem 3.1] and the error bounds follow from the bounds on $\mathcal{V}_{0, \infty}(H)$ in Lemma 3.15. The Wronskian identity is a direct consequence of the chain rule.

Lemma 3.17 There exists a constant $A_{2} \neq 0$ such that $s_{1}=A_{2} w_{1}$, where $w_{1}$ is defined in (3.176) and $s_{1}$ is defined in (3.58).

Proof Note that both, $w_{1}$ and $s_{1}$ are recessive as $y \rightarrow \infty$. Since the space of solutions which are recessive at $y \rightarrow \infty$ is one-dimensional, we conclude that $s_{1}$ and $w_{1}$ are linearly dependent.

In view of Lemma 3.17 we define

$$
s_{2}:=\frac{1}{A_{2} \mathfrak{W}\left(w_{1}, w_{2}\right)} w_{2},
$$

where $w_{2}$ is as in (3.177). Note that this implies that

$$
\mathfrak{W}\left(s_{1}, s_{2}\right)=1
$$


Lemma 3.18 Let $\tilde{\lambda} \in\left[\Xi^{2}+1, \infty\right)$ and let $s_{2}$ as in (3.181). Then, $g_{p}$ defined in (3.129) satisfies

$$
\int_{0}^{\infty} g_{p}(y)^{2}\left(1-x(y)^{2}\right) \Delta_{x} \mathrm{~d} y \lesssim m
$$

Proof Analogous to the proof of Lemma 3.13 we first estimate

$$
\begin{aligned}
\int_{0}^{\infty} s_{2}^{2}(y) & \left(\int_{y}^{\infty} s_{1}^{2}(\tilde{y}) F(\tilde{y}) \mathrm{d} \tilde{y}\right)^{2}\left(1-x(y)^{2}\right) \Delta_{x} \mathrm{~d} y \\
= & \int_{0}^{\infty} \frac{w_{2}^{2}(y)}{\mathfrak{W}\left(w_{1}, w_{2}\right)^{2}}\left(\int_{y}^{\infty} w_{1}(\tilde{y}) s_{1}(\tilde{y}) F(\tilde{y}) \mathrm{d} \tilde{y}\right)^{2} \\
& \times\left(1-x(y)^{2}\right) \Delta_{x}(y) \mathrm{d} y .
\end{aligned}
$$

Now, we use Proposition 3.6 and standard bounds on Airy functions from Appendix A.1, as well as Lemma 3.16 to obtain

$$
\begin{aligned}
& \left|w_{1}(y)\right| \lesssim\left|E_{\mathrm{Ai}}^{-1}\left(\lambda^{\frac{1}{3}} \varsigma(y)\right) \frac{M_{\mathrm{Ai}}\left(\lambda^{\frac{1}{3}} \varsigma(y)\right)}{\hat{W}_{1}^{\frac{1}{4}}(y)}\right| \lesssim E_{\mathrm{Ai}}^{-1}\left(\lambda^{\frac{1}{3}} \varsigma(y)\right) \tilde{\lambda}^{\frac{1}{6}}, \\
& \left|w_{2}(y)\right| \lesssim\left|E_{\mathrm{Ai}}\left(\lambda^{\frac{1}{3}} \varsigma(y)\right) \frac{M_{\mathrm{Ai}}\left(\lambda^{\frac{1}{3}} \varsigma(y)\right)}{\hat{W}_{1}^{\frac{1}{4}}(y)}\right| \lesssim E_{\mathrm{Ai}}\left(\lambda^{\frac{1}{3}} \varsigma(y)\right) \tilde{\lambda}^{\frac{1}{6}} .
\end{aligned}
$$

Now, plugging these estimates into (3.184) and using that $E_{\mathrm{Ai}}^{-1}\left(\lambda^{\frac{1}{3}} \zeta(y)\right)$ is a decreasing function, we conclude

$$
\begin{aligned}
& \int_{0}^{\infty} s_{2}^{2}(y)\left(\int_{y}^{\infty} s_{1}^{2}(\tilde{y}) F(\tilde{y}) \mathrm{d} \tilde{y}\right)^{2}\left(1-x(y)^{2}\right) \Delta_{x} \mathrm{~d} y \\
& \quad \lesssim \int_{0}^{\infty} \frac{\tilde{\lambda}^{\frac{2}{3}}}{\mathfrak{W}\left(w_{1}, w_{2}\right)^{2}}\left(\int_{y}^{\infty} s_{1}(\tilde{y}) F(\tilde{y}) \mathrm{d} \tilde{y}\right)^{2}\left(1-x(y)^{2}\right) \Delta_{x} \mathrm{~d} y \\
& \lesssim \frac{\tilde{\lambda}^{\frac{2}{3}}}{\mathfrak{W}\left(w_{1}, w_{2}\right)^{2}} \int_{0}^{\infty}\left(1-x(y)^{2}\right) \Delta_{x} \mathrm{~d} y \\
& \quad \times \int_{0}^{\infty} s_{1}^{2}(y)\left(1-x(y)^{2}\right) \Delta_{x} \mathrm{~d} y \int_{0}^{\infty} F^{2}(y) \frac{1}{\left(1-x(y)^{2}\right) \Delta_{x}} \mathrm{~d} y \\
& \lesssim \frac{\tilde{\lambda}^{\frac{2}{3}} m^{2}}{\mathfrak{W}\left(w_{1}, w_{2}\right)^{2}} .
\end{aligned}
$$


For the second term, we argue similarly and estimate

$$
\begin{aligned}
\int_{0}^{\infty} s_{1}^{2}(y) & \left(\int_{0}^{y} s_{2}(\tilde{y}) s_{1}(\tilde{y}) F(\tilde{y}) \mathrm{d} \tilde{y}\right)^{2}\left(1-x(y)^{2}\right) \Delta_{x} \mathrm{~d} y \\
& \leq\left(\int_{0}^{\infty}\left|s_{2}(\tilde{y}) s_{1}(\tilde{y}) F(\tilde{y})\right| \mathrm{d} \tilde{y}\right)^{2} \int_{0}^{\infty} s_{1}^{2}(y)\left(1-x(y)^{2}\right) \Delta_{x} \mathrm{~d} y \\
& =\frac{1}{2}\left(\int_{0}^{\infty}\left|s_{2}(\tilde{y}) s_{1}(\tilde{y}) F(\tilde{y})\right| \mathrm{d} \tilde{y}\right)^{2} \\
& \lesssim \frac{\tilde{\lambda}^{\frac{2}{3}}}{\mathfrak{W}\left(w_{1}, w_{2}\right)^{2}}\left(\int_{0}^{\infty}|F| \mathrm{d} \tilde{y}\right)^{2} \lesssim \frac{\tilde{\lambda}^{\frac{2}{3}} m^{2}}{\mathfrak{W}\left(w_{1}, w_{2}\right)^{2}}
\end{aligned}
$$

Thus, we conclude

$$
\int_{0}^{\infty} g_{p}(y)^{2}\left(1-x(y)^{2}\right) \Delta_{x} \mathrm{~d} y \lesssim \frac{\tilde{\lambda}^{\frac{2}{3}} m^{2}}{\mathfrak{W}\left(w_{1}, w_{2}\right)^{2}} \lesssim \frac{\tilde{\lambda}^{\frac{2}{3}} m^{2}}{\lambda^{\frac{2}{3}}} \lesssim m^{\frac{2}{3}} \lesssim m .
$$

\section{The radial o.d.e. on the exterior}

We will now derive for which frequency parameters $(\omega, m, \ell)$ the poles of the interior scattering operator at $\omega=\omega_{-} m$ coincide with frequency parameters which are exposed to stable trapping on the black hole exterior. This allows us then to define the set $\mathscr{P}_{\text {Blow-up in Sect. 5.1. Thus, we will analyze the radial }}$ o.d.e. at frequency $\omega=\omega_{-} m$.

\subsection{Resonance: Radial o.d.e. at interior scattering poles allows for stable trapping}

We will first determine the range of angular eigenvalues $\lambda_{m \ell}\left(a m \omega_{-}\right)$at the interior scattering poles $\omega=\omega_{-} m$ for which the radial o.d.e. admits stable trapping. Recall from (2.48) that the normalized high frequency part of the potential with $\omega=\omega_{-} m$ is given by

$$
V_{\text {main }}=\frac{\Delta}{\left(r^{2}+a^{2}\right)^{2}}\left(\frac{\lambda_{m \ell}\left(a \omega_{-} m\right)}{m^{2}}+\omega_{-}^{2} a^{2}-2 a \omega_{-} \Xi\right)-\left(\omega_{-}-\omega_{r}\right)^{2} .
$$


Note that

$$
\begin{aligned}
& V_{\text {main }} \rightarrow-\left(\omega_{-}-\omega_{+}\right)^{2}<0 \text { as } r \rightarrow r_{+}, \\
& V_{\text {main }} \rightarrow l^{-2}\left(\frac{\lambda_{m \ell}\left(a \omega_{-} m\right)}{m^{2}}+\omega_{-}^{2} a^{2}-2 a \omega_{-} \Xi\right)-\omega_{-}^{2} \text { as } r \rightarrow \infty, \\
& V_{\text {main }}\left(r_{\text {cut }}\right) \geq\left(l^{-2}+3 \frac{M^{2}}{\Xi}\left(\frac{9 M^{2}}{\Xi^{2}}+a^{2}\right)^{-2}\right) \\
& \left(\frac{\lambda_{m \ell}\left(a \omega_{-} m\right)}{m^{2}}+\omega_{-}^{2} a^{2}-2 a \omega_{-} \Xi\right)-\omega_{-}^{2},
\end{aligned}
$$

where $r_{\text {cut }}:=\frac{3 M}{\Xi}$. Remark that (4.4) follows from (using $l^{2}=a^{2}+l^{2} \Xi$ )

$$
\begin{aligned}
\frac{\Delta(r)}{\left(r^{2}+a^{2}\right)^{2}} & =l^{-2} \frac{\left(r^{2}+a^{2}\right)\left(r^{2}+l^{2}\right)}{\left(r^{2}+a^{2}\right)^{2}}-\frac{2 M r}{\left(a^{2}+r^{2}\right)^{2}} \\
& =l^{-2}+\frac{\Xi\left(r^{2}+a^{2}\right)-2 M r}{\left(a^{2}+r^{2}\right)^{2}},
\end{aligned}
$$

together with $\Xi\left(r_{\text {cut }}^{2}+a^{2}\right)-2 M r_{\text {cut }}=\frac{3 M^{2}}{\Xi}+a^{2} \Xi$.

For the potential $V_{\text {main }}$ to admit a region of stable trapping, we require that $V_{\text {main }}$ has two roots $r_{1}<r_{2}$, see already Fig. 7. A sufficient condition for that is that the angular eigenvalues $\lambda_{m \ell}\left(a \omega_{-} m\right) m^{-2}$ are such that (4.3) is negative and (4.4) is positive. In this case, we denote with $r_{1}=r_{1}\left(\lambda_{m \ell}(a \omega-m) m^{-2}, \mathfrak{p}\right)<$ $r_{2}=r_{2}\left(\lambda_{m \ell}\left(a \omega_{-} m\right) m^{-2}, \mathfrak{p}\right)$ the two largest roots. (We will show later that indeed, these are the only roots for $r \geq r_{+}$.) This leads us to define the following range of angular eigenvalues

$$
E:=\bigsqcup_{\mathfrak{p} \in \mathscr{P}}\{\mathfrak{p}\} \times E_{\mathfrak{p}}=\bigsqcup_{\mathfrak{p} \in \mathscr{P}}\{\mathfrak{p}\} \times\left(\mu_{0}(\mathfrak{p}), \mu_{1}(\mathfrak{p})\right)
$$

where

$$
\begin{aligned}
E_{\mathfrak{p}}:= & \left\{\tilde { \mu } \in ( \Xi ^ { 2 } , \omega _ { - } ^ { 2 } ( l ^ { 2 } - a ^ { 2 } ) + 2 a \omega _ { - } \Xi ) : \text { Every } \mu \in \left[\tilde{\mu}, \omega_{-}^{2}\left(l^{2}-a^{2}\right)\right.\right. \\
& \left.+2 a \omega_{-} \Xi\right) \text { satisfies } \\
& \left(l^{-2}+3 \frac{M^{2}}{\Xi}\left(\frac{9 M^{2}}{\Xi^{2}}+a^{2}\right)^{-2}\right)\left(\mu+\omega_{-}^{2} a^{2}-2 a \omega_{-} \Xi\right)-\omega_{-}^{2}>0 \\
& \Gamma\left(\Delta^{-1}\right)\left(\mu+a^{2} \omega_{-}^{2}-2 a \omega_{-} \Xi\right)-\Gamma\left(\frac{\left(r^{2}+a^{2}\right)^{2}}{\Delta^{2}}\left(\omega_{-}-\omega_{r}\right)^{2}\right) \\
& \left.<-\frac{\omega_{-}}{a} l^{4} r^{-4} \text { for } r \geq r_{2}(\mu, \mathfrak{p})\right\} .
\end{aligned}
$$


Remark that the last condition in (4.7) will be used in Lemma 5.3 while the other conditions in (4.7) guarantee that $V_{\text {main }}$ has two roots. Here, we also recall the definition of $\Gamma=\frac{\partial}{\partial \vartheta}$ in (2.11), where $\vartheta=a \omega_{-}$.

By construction, $E_{\mathfrak{p}}$ is connected. Indeed, note that if $\tilde{\mu}_{1}, \tilde{\mu}_{2} \in E_{\mathfrak{p}}$, then every $\tilde{\mu}_{3}$ with $\tilde{\mu}_{1} \leq \tilde{\mu}_{3} \leq \tilde{\mu}_{2}$ satisfies $\tilde{\mu}_{3} \in E_{\mathfrak{p}}$. Thus, $E_{\mathfrak{p}}$ is a (a priori possibly empty) bounded interval. We define $\mu_{0}(\mathfrak{p}):=\inf E_{\mathfrak{p}}, \mu_{1}(\mathfrak{p}):=\sup E_{\mathfrak{p}}$. We will show that $E$ is a fiber bundle. To do so we first show that $E_{\mathfrak{p}}$ is open and non-empty.

Lemma 4.1 For any $\mathfrak{p} \in \mathscr{P}$, the set $E_{\mathfrak{p}}$ defined in (4.7) is a non-empty bounded open interval.

Proof First, we will show that

$$
\Xi^{2}<\omega_{-}^{2}\left(l^{2}-a^{2}\right)+2 a \omega_{-} \Xi
$$

which in turn follows from

$$
r_{-}^{2}<a l
$$

To see that $r_{-}^{2}<a l$ holds true, we write $\Delta(r)$ in terms of $r_{-}$. More precisely, from $\Delta\left(r_{-}\right)=0$ we have

$$
2 M=r_{-}^{-1}\left(a^{2}+r_{-}^{2}\right)\left(1+\frac{r_{-}^{2}}{l^{2}}\right)
$$

from which we obtain

$$
\Delta(r)=\left(r^{2}+a^{2}\right)\left(1+\frac{r^{2}}{l^{2}}\right)-\frac{r}{r_{-}}\left(a^{2}+r_{-}^{2}\right)\left(1+\frac{r_{-}^{2}}{l^{2}}\right) .
$$

After a polynomial long division, this reduces to

$$
\Delta(r)=l^{-2}\left(r-r_{-}\right)\left(r^{3}+r^{2} r_{-}+r\left(r_{-}^{2}+a^{2}+l^{2}\right)-a^{2} l^{2} r_{-}^{-1}\right) .
$$

Hence,

$$
0>\partial_{r} \Delta\left(r_{-}\right)=l^{-2} r_{-}^{-1}\left(3 r_{-}^{4}+r_{-}^{2} a^{2}+r_{-}^{2} l^{2}-a^{2} l^{2}\right)
$$

implies

$$
3 r_{-}^{4}<a^{2} l^{2}
$$


from which

$$
r_{-}^{2}<a l
$$

follows.

Note that for $\mu=\omega_{-}^{2}\left(l^{2}-a^{2}\right)+2 a \omega_{-} \Xi$ we have

$$
\begin{aligned}
& \left(l^{-2}+3 \frac{M^{2}}{\Xi}\left(\frac{9 M^{2}}{\Xi^{2}}+a^{2}\right)^{-2}\right)\left(\mu+\omega_{-}^{2} a^{2}-2 a \omega_{-} \Xi\right)-\omega_{-}^{2} \\
& \quad=\left(l^{-2}+3 \frac{M^{2}}{\Xi}\left(\frac{9 M^{2}}{\Xi^{2}}+a^{2}\right)^{-2}\right) \omega_{-}^{2} l^{2}-\omega_{-}^{2} \\
& \quad=3 \frac{M^{2}}{\Xi}\left(\frac{9 M^{2}}{\Xi^{2}}+a^{2}\right)^{-2} \omega_{-}^{2} l^{2}>0 .
\end{aligned}
$$

Thus, for $\mu<\omega_{-}^{2}\left(l^{2}-a^{2}\right)+2 a \omega_{-} \Xi$ and $\omega_{-}^{2}\left(l^{2}-a^{2}\right)+2 a \omega_{-} \Xi-\mu$ sufficiently small, we have

$$
\left(l^{-2}+3 \frac{M^{2}}{\Xi}\left(\frac{9 M^{2}}{\Xi^{2}}+a^{2}\right)^{-2}\right)\left(\mu+\omega_{-}^{2} a^{2}-2 a \omega_{-} \Xi\right)-\omega_{-}^{2}>0 .
$$

Now, note that for $\mu=\lambda_{m \ell}\left(a \omega_{-} m\right) m^{-2}=\omega_{-}^{2}\left(l^{2}-a^{2}\right)+2 a \omega_{-} \Xi$, we have that $V_{\text {main }} \rightarrow 0$ as $r \rightarrow \infty$. Thus, the largest root $r_{2}$ satisfies $r_{2}(\mu, \mathfrak{p}) \rightarrow+\infty$ as $\mu \rightarrow \omega_{-}^{2}\left(l^{2}-a^{2}\right)+2 a \omega_{-} \Xi$ from below. Hence, the claim follows from the fact that to highest order in $r$, the last condition in (4.7) is fulfilled. More precisely,

$$
\begin{aligned}
& \lim _{r \rightarrow \infty} r^{4}\left(\Gamma\left(\Delta^{-1}\right)\left(\mu+a^{2} \omega_{-}^{2}-2 a \omega_{-} \Xi\right)\right. \\
& \left.\quad-\Gamma\left(\frac{\left(r^{2}+a^{2}\right)^{2}}{\Delta^{2}}\left(\omega_{-}-\omega_{r}\right)^{2}\right)\right)=-l^{4} \Gamma\left(\omega_{-}^{2}\right)=\frac{-2 \omega_{-}}{a} l^{4}<0,
\end{aligned}
$$

where we used $\Gamma\left(a \omega_{-}\right)=1$ and $\Gamma(a)=0$.

From (4.7) it also follows that $\mu_{0}$ and $\mu_{1}$ are manifestly continuous functions on $\mathscr{P}$. Hence, $E$ is a (topological) fiber bundle. Now, also note that $E$ is trivial with global trivialization $\varphi_{E}: E \rightarrow \mathscr{P} \times(0,1),(\mathfrak{p}, \mu) \mapsto\left(\mathfrak{p}, \frac{\mu}{\mu_{1}-\mu_{0}}-\frac{\mu_{0}}{\mu_{1}-\mu_{0}}\right)$ and we find (using this trivialization) two global sections

$$
\sigma_{1} \in \Gamma(E) \quad \text { and } \quad \sigma_{2} \in \Gamma(E) \text { with } \sigma_{1}(\mathfrak{p})<\sigma_{2}(\mathfrak{p})
$$

for all $\mathfrak{p} \in \mathscr{P}$ (in mild abuse of notation). For definiteness, we take

$$
\sigma_{1}(\mathfrak{p}):=\varphi_{E}^{-1}\left(\mathfrak{p}, \frac{1}{2}\right) \text { and } \sigma_{2}(\mathfrak{p}):=\varphi_{E}^{-1}\left(\mathfrak{p}, \frac{3}{4}\right) \text {. }
$$


Having constructed $\sigma_{1}$ and $\sigma_{2}$, we will now show the existence of exactly two turning points $r_{1}<r_{2}$ of $V_{\text {main }}$.

Lemma 4.2 Let $m^{-2} \lambda_{m \ell}\left(a \omega_{-} m\right) \in\left(\sigma_{1}, \sigma_{2}\right)$ as chosen in (4.18). Then, $V_{\text {main }}$ has a maximum $r_{\max } \in\left(r_{+}, \infty\right)$, and two roots $r_{1}, r_{2}$ with $r_{+}<r_{1}<r_{\max }<$ $r_{2}<\infty$ such that

$$
\begin{aligned}
& V_{\text {main }}>0 \text { for } r \in\left(r_{1}, r_{2}\right) \text {, } \\
& V_{\text {main }}<0 \text { for } r \in\left[r_{+}, \infty\right) \backslash\left[r_{1}, r_{2}\right]
\end{aligned}
$$

and $r_{2}-r_{1} \gtrsim 1$.

Proof By construction of $\sigma_{1}$ and $\sigma_{2}$, for any $m^{-2} \lambda_{m \ell} \in\left(\sigma_{1}, \sigma_{2}\right)$, the potential $V_{\text {main }}$ has a maximum and satisfies

$$
\lim _{r \rightarrow \infty} V_{\text {main }}<0, V_{\text {main }}\left(r=r_{+}\right)<0 \text { and } V_{\text {main }}\left(r=r_{\text {cut }}\right)>0,
$$

where $r_{\text {cut }}=\frac{3 M}{\Xi}$. See also [65, Lemma 3.1].

We will show now that $V_{\text {main }}$ has exactly two roots in $\left[r_{+}, \infty\right)$ from which (4.20) and (4.21) follow. Indeed, in view of the above, $V_{\text {main }}$ either has two or four roots in $\left[r_{+}, \infty\right)$. To exclude the case of four roots, it suffices to exclude the case of three critical points in $\left[r_{+}, \infty\right)$. To see this, note that

$$
\begin{aligned}
\frac{\mathrm{d} V_{\text {main }}}{\mathrm{d} r}= & \frac{\left(-2 \Xi r^{3}+6 M r^{2}-2 \Xi a^{2} r-2 M a^{2}\right) m^{-2}\left(\lambda_{m \ell}\left(a \omega_{-} m\right)+a^{2} \omega_{-}^{2}-2 a \omega_{-} \Xi\right)}{\left(r^{2}+a^{2}\right)^{3}} \\
& +\frac{4 a r \Xi\left(\omega_{r}-\omega_{-}\right)\left(r^{2}+a^{2}\right)}{\left(r^{2}+a^{2}\right)^{3}}
\end{aligned}
$$

has at most three real roots, one of which is in $\left[r_{+}, \infty\right)$ in view of the construction above. Indeed, one other root has to lie in $\left(-\infty, r_{-}\right]$as

$$
\lim _{r \rightarrow-\infty} \frac{\mathrm{d} V_{\text {main }}}{\mathrm{d} r}>0 \text { and } \frac{\mathrm{d} V_{\text {main }}}{\mathrm{d} r}\left(r=r_{-}\right)=\frac{\partial_{r} \Delta\left(r_{-}\right)}{\left(r_{-}^{2}+a^{2}\right)^{2}}<0 .
$$

Thus, $V_{\text {main }}$ has at least one and at most two critical points in $\left[r_{+}, \infty\right)$ from which we deduce (4.20) and (4.21).

\subsection{Fundamental pairs of solutions associated to trapping}

We will now define various solutions to the radial o.d.e. associated to the boundary and to the turning points. Note that the turning points define the transition from the trapping region to the semiclassically forbidden region. 


\subsubsection{Solutions associated to the boundary}

We first define the associated solution to the radial o.d.e. (2.43) which satisfies the Dirichlet boundary conditions at $r^{*}=\frac{\pi}{2} l$.

Definition 4.1 For all frequencies $\omega \in \mathbb{R}, m \in \mathbb{Z}, \ell \in \mathbb{Z}_{\geq|m|}$ we define the solution $u_{\infty}$ as the unique solution to (2.43) satisfying

$$
\begin{aligned}
& u_{\infty}\left(\frac{\pi}{2} l\right)=0, \\
& u_{\infty}^{\prime}\left(\frac{\pi}{2} l\right)=1,
\end{aligned}
$$

where we recall that ${ }^{\prime}=\frac{\mathrm{d}}{\mathrm{d} r^{*}}$.

We now define solutions associated to the event horizon $\mathcal{H}^{+}$. The limit $r^{*} \rightarrow$ $-\infty$ of the radial o.d.e. (2.43) constitutes a regular singular point. In particular, in view of [94, Theorem 2.2, Chapter 6] (set $f=\left(\omega-\omega_{+} m\right)^{2}$ and $g=$ $V-\omega^{2}-\left(\omega-\omega_{+} m\right)^{2}=O_{\omega, m, \ell}(\Delta)$ as $\left.r^{*} \rightarrow-\infty\right)$ we can define the following unique solutions to (2.43).

Definition 4.2 For all frequencies $\omega \in \mathbb{R}, m \in \mathbb{Z}, \ell \in \mathbb{Z}_{\geq|m|}$ we also define $u_{\mathcal{H}^{+}}$and $u_{\mathcal{H}^{-}}$as the unique solutions to (2.43) satisfying

$$
\begin{aligned}
& u_{\mathcal{H}^{+}}=e^{-i\left(\omega-\omega_{+} m\right) r^{*}}+O_{\omega, m, \ell}(\Delta) \text { as } r^{*} \rightarrow-\infty, \\
& u_{\mathcal{H}^{-}}=e^{i\left(\omega-\omega_{+} m\right) r^{*}}+O_{\omega, m, \ell}(\Delta) \text { as } r^{*} \rightarrow-\infty .
\end{aligned}
$$

Remark 4.1 Equivalently, we can define $u_{\mathcal{H}^{+}}$(and similarly $u_{\mathcal{H}^{+}}$) as the unique solution to the Volterra integral equation

$$
\begin{aligned}
u_{\mathcal{H}^{+}}\left(r^{*}\right)= & e^{-i\left(\omega-\omega_{+} m\right) r^{*}}+\int_{-\infty}^{r^{*}} \frac{\sin \left(\left(\omega-\omega_{+} m\right)\left(r^{*}-y\right)\right)}{\omega-\omega_{+} m} \\
& \times\left(V(y)-\omega^{2}+\left(\omega-\omega_{+} m\right)^{2}\right) u_{\mathcal{H}^{+}}(y) \mathrm{d} y,
\end{aligned}
$$

where $V$ is as in (2.44) and we note that $V(y)-\omega^{2}+\left(\omega-\omega_{+} m\right)^{2}=O_{\omega, m, \ell}(\Delta)$ as $r^{*} \rightarrow-\infty$. Existence and uniqueness is standard, see e.g. [94, Theorem 10.1, Chapter 6]. Note also that the fact that $u_{\mathcal{H}^{+}}$defined by (4.29) indeed solves the o.d.e. can be checked explicitly.

\subsubsection{Solutions associated to turning points at interior scattering poles}

For the solutions associated to the turning points we only consider the radial o.d.e. for $\omega=\omega_{-} m$ which we recall from (2.47) as

$$
-u^{\prime \prime}+\left(m^{2} V_{\text {main }}+V_{1}\right) u=0 .
$$




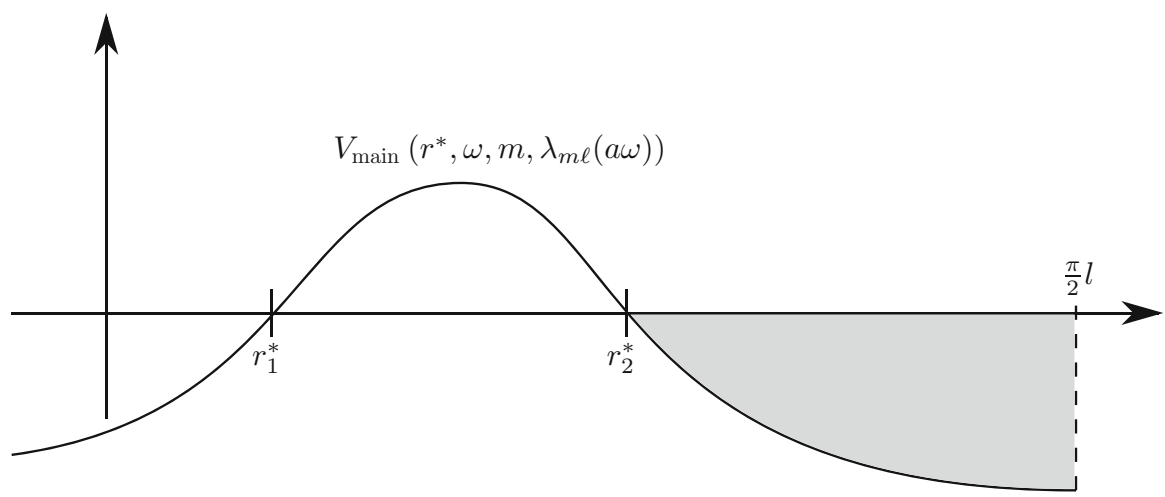

Fig. 7 Rough shape of the potential $V_{\text {main }}$ and the turning points $r_{1}^{*}$ and $r_{2}^{*}$

Throughout Sect. 4.2.2 we assume that $\sigma_{1} \leq \lambda_{m \ell} m^{-2} \leq \sigma_{2}$ and in view of Lemma 4.2 we denote the turning points of $V_{\text {main }}$ with $r_{1}^{*}:=r^{*}\left(r_{1}\right)<$ $r^{*}\left(r_{2}\right)=: r_{2}^{*}$, where we recall that they do not coalesce for $\sigma_{1} \leq \lambda_{m \ell} m^{-2} \leq \sigma_{2}$. We will now define solutions associated to the turning points $r_{1}^{*}$ and $r_{2}^{*}$ as illustrated in Fig. 7. We will closely follow [94, Chapter 11, §3], see also [92] for the original publication. We begin by defining new variables $\xi_{1}$ and $\xi_{2}$ in the following which will by construction solve

$$
\xi_{1}\left(\frac{\mathrm{d} \xi_{1}}{\mathrm{~d} r^{*}}\right)^{2}=V_{\text {main }}, \quad \xi_{2}\left(\frac{\mathrm{d} \xi_{2}}{\mathrm{~d} r^{*}}\right)^{2}=V_{\text {main }} .
$$

Definition 4.3 For some fixed $\epsilon=\epsilon(\mathfrak{p})>0$ sufficiently small depending smoothly on $\mathfrak{p}$, we define

$$
\begin{aligned}
& \xi_{1}\left(r^{*}, m\right):= \begin{cases}-\left(\frac{3}{2} \int_{r^{*}}^{r_{1}^{*}}\left(-V_{\text {main }}\right)^{\frac{1}{2}} \mathrm{~d} y\right)^{\frac{2}{3}} & r^{*} \in\left(-\infty, r_{1}^{*}\right), \\
\left(\frac{3}{2} \int_{r_{1}^{*}}^{r^{*}} V_{\text {main }}^{\frac{1}{2}} \mathrm{~d} y\right)^{\frac{2}{3}} & r^{*} \in\left[r_{1}^{*}, r_{2}^{*}-\epsilon\right],\end{cases} \\
& \xi_{2}\left(r^{*}, m\right):= \begin{cases}\left(\frac{3}{2} \int_{r^{*}}^{r_{2}^{*}} V_{\text {main }}^{\frac{1}{2}} \mathrm{~d} y\right)^{\frac{2}{3}} & r^{*} \in\left[r_{1}^{*}+\epsilon, r_{2}^{*}\right), \\
-\left(\frac{3}{2} \int_{r_{2}^{*}}^{r^{*}}\left(-V_{\text {main }}\right)^{\frac{1}{2}} \mathrm{~d} y\right)^{\frac{2}{3}} & r^{*} \in\left[r_{2}^{*}, \frac{\pi}{2} l\right],\end{cases} \\
& \hat{f}_{1}:=\frac{V_{\text {main }}}{\xi_{1}}=\left(\frac{\mathrm{d} \xi_{1}}{\mathrm{~d} r^{*}}\right)^{2} \quad \text { for } r^{*} \in\left(-\infty, r_{2}^{*}-\epsilon\right], \\
& \hat{f}_{2}:=\frac{V_{\text {main }}}{\xi_{2}}=\left(\frac{\mathrm{d} \xi_{1}}{\mathrm{~d} r^{*}}\right)^{2} \quad \text { for } r^{*} \in\left[r_{1}^{*}+\epsilon, \frac{\pi}{2} l\right],
\end{aligned}
$$


where we note that $r^{*} \mapsto \xi_{1}$ is monotonically increasing and $r^{*} \mapsto \xi_{2}$ is monotonically decreasing. In particular, we choose $\epsilon(\mathfrak{p})>0$ sufficiently small to ensure that

$$
\frac{\xi_{2}\left(r_{1}^{*}+2 \epsilon\right)}{\xi_{1}\left(r_{1}^{*}+2 \epsilon\right)} \geq 3
$$

As in $[94$, Chapter 11, §3] we note that with the above definitions the new unknowns $W_{1}:=\left(\frac{\mathrm{d} \xi_{1}}{\mathrm{~d} r^{*}}\right)^{\frac{1}{2}} u$ and $W_{2}:=\left(-\frac{\mathrm{d} \xi_{2}}{\mathrm{~d} r^{*}}\right)^{\frac{1}{2}} u$ respectively solve

$$
\begin{aligned}
& \frac{\mathrm{d}^{2} W_{1}}{\mathrm{~d} \xi_{1}^{2}}=\left(m^{2} \xi_{1}+\Psi_{1}\right) W_{1}, \\
& \frac{\mathrm{d}^{2} W_{2}}{\mathrm{~d} \xi_{2}^{2}}=\left(m^{2} \xi_{2}+\Psi_{2}\right) W_{2}
\end{aligned}
$$

for the error functions

$$
\Psi_{1}=\frac{1}{\hat{f}_{1}^{\frac{1}{4}}} \frac{\mathrm{d}^{2} \hat{f}_{1}^{\frac{1}{4}}}{\mathrm{~d} \xi_{1}^{2}}+\frac{V_{1}}{\hat{f}_{1}}=-\frac{1}{\hat{f}_{1}^{\frac{3}{4}}} \frac{\mathrm{d}^{2}}{\mathrm{~d} r^{* 2}}\left(\frac{1}{\hat{f}_{1}^{\frac{1}{4}}}\right)+\frac{V_{1}}{\hat{f}_{1}}
$$

and

$$
\Psi_{2}=\frac{1}{\hat{f}_{2}^{\frac{1}{4}}} \frac{\mathrm{d}^{2} \hat{f}_{2}^{\frac{1}{4}}}{\mathrm{~d} \xi_{2}^{2}}+\frac{V_{1}}{\hat{f}_{2}}=-\frac{1}{\hat{f}_{2}^{\frac{3}{4}}} \frac{\mathrm{d}^{2}}{\mathrm{~d} r^{* 2}}\left(\frac{1}{\hat{f}_{2}^{\frac{1}{4}}}\right)+\frac{V_{1}}{\hat{f}_{2}} .
$$

As in $[94$, Chapter $11, \S 3]$ this can be equivalently written as

$$
\Psi_{1}=\frac{5}{16 \xi_{1}^{2}}+\left[4 V_{\text {main }} \frac{\mathrm{d}^{2}}{\mathrm{~d} r^{* 2}} V_{\text {main }}-5\left(\frac{\mathrm{d}}{\mathrm{d} r^{*}} V_{\text {main }}\right)^{2}\right] \frac{\xi_{1}}{16 V_{\text {main }}^{3}}+\frac{\xi_{1} V_{1}}{V_{\text {main }}},
$$

and

$$
\Psi_{2}=\frac{5}{16 \xi_{2}^{2}}+\left[4 V_{\text {main }} \frac{\mathrm{d}^{2}}{\mathrm{~d} r^{* 2}} V_{\text {main }}-5\left(\frac{\mathrm{d}}{\mathrm{d} r^{*}} V_{\text {main }}\right)^{2}\right] \frac{\xi_{2}}{16 V_{\text {main }}^{3}}+\frac{\xi_{2} V_{1}}{V_{\text {main }}} .
$$

Lemma 4.3 The functions $\left(-\infty, r_{2}^{*}-\epsilon\right] \ni r^{*} \mapsto \xi_{1} /\left(r^{*}-r_{1}^{*}\right),\left[r_{1}^{*}+\epsilon, \frac{\pi l}{2}\right] \ni$ $r^{*} \mapsto \xi_{2} /\left(r_{2}^{*}-r^{*}\right)$ are smooth positive functions in their respective domains. 
Moreover, $\left(-\infty, r_{2}^{*}-\epsilon\right] \ni r^{*} \mapsto \xi_{1}\left(r^{*}\right)\left(\operatorname{resp} .\left[r_{1}^{*}+\epsilon, \frac{\pi l}{2}\right] \ni r^{*} \mapsto \xi_{2}\left(r^{*}\right)\right)$ is a strictly increasing (resp. decreasing) smooth function.

The error control functions $\left(-\infty, r_{2}^{*}-\epsilon\right] \ni r^{*} \mapsto \Psi_{1}\left(r^{*}\right)$ and $\left[r_{1}^{*}+\epsilon, \frac{\pi l}{2}\right] \ni$ $r^{*} \mapsto \Psi_{2}\left(r^{*}\right)$ are also smooth functions. Moreover, $\xi_{1}, \xi_{2}, \Psi_{1}, \Psi_{2}$ depend smoothly on $\left(r^{*}, \mathfrak{p}\right)$.

Proof Mutatis mutandis, this follows from [94, Chapter 11, §3, Lemma 3.1]. For convenience of the reader we will give a proof for the case of $\xi_{1}$ and $\Psi_{1}$ following the proof of [94, Chapter 11, §3, Lemma 3.1]. We first write $p\left(r^{*}\right):=\frac{V_{\text {main }}}{r^{*}-r_{1}^{*}}$ which is smooth as $V_{\text {main }}$ has a simple root at $r_{1}^{*}$. We also set $q\left(r^{*}\right):=\left(r^{*}-r_{1}^{*}\right)^{-\frac{3}{2}} \int_{r_{1}^{*}}^{r^{*}}\left(y-r_{1}^{*}\right)^{\frac{1}{2}} p(y) \mathrm{d} y$ for $r^{*}>r_{1}^{*}$ such that by construction, $\frac{\xi_{1}\left(r^{*}\right)}{r^{*}-r_{1}^{*}}=\left(\frac{3}{2} q\left(r^{*}\right)\right)^{\frac{2}{3}}$. Moreover, $q$ is also smooth with $n$-th derivative $q^{(n)}\left(r^{*}\right)=\left(r^{*}-r_{1}^{*}\right)^{\frac{2 n+1}{2}} \int_{r_{1}^{*}}^{r^{*}}\left(y-r_{1}^{*}\right)^{\frac{2 n-1}{2}} p^{(n)}(y) \mathrm{d} y$ which follows from integrating by parts. By the mean value theorem we have that for every $n$, the derivative $q^{(n)}\left(r^{*}\right)$ has a right limit $r^{*} \rightarrow r_{1}^{*}$ and in particular, $\lim _{r^{*} \rightarrow r_{1}^{*}} q\left(r^{*}\right)=\frac{2}{3} p\left(r_{1}^{*}\right) \neq 0$, where the last property follows from the fact that $V_{\text {main }}$ has a simple root at $r_{1}^{*}$. Thus, $\frac{\xi_{1}\left(r^{*}\right)}{r^{*}-r_{1}^{*}}=\left(\frac{3}{2} q\left(r^{*}\right)\right)^{\frac{2}{3}}$ is positive and extends smoothly across $r^{*}=r_{1}^{*}$. Arguing completely analogously for the region $r^{*}<r_{1}^{*}$ and noting that by construction the left and right derivatives agree at $r^{*}=r_{1}^{*}$, we obtain that $\frac{\xi_{1}\left(r^{*}\right)}{r^{*}-r_{1}^{*}}$ is a smooth positive function on $\left(-\infty, r_{2}^{*}-\epsilon\right]$.

Moreover, as $r_{1}^{*}$ and $V_{\text {main }}$ depend smoothly on $\left(r^{*}, \mathfrak{p}\right)$, we also have that $\xi_{1}$ depends smoothly on $\left(r^{*}, \mathfrak{p}\right)$. Now, we note that $\hat{f}_{1}\left(r^{*}\right)=p\left(r^{*}\right)^{2}\left(\frac{3}{2} q\left(r^{*}\right)\right)^{-\frac{2}{3}}$ such that $\hat{f}_{1}$ is a smooth positive function of $r^{*}$ and $\hat{f}_{1}$ also depends smoothly on $\mathfrak{p}$ in view of the above properties shown about $p$ and $q$. In view of (4.39), this finally shows that $\Psi_{1}$ is a smooth function of $r^{*}$ (and of $\xi_{1}$ ) and also depends smoothly on $\mathfrak{p}$. The claim about $\xi_{2}$ and $\Psi_{2}$ follows completely analogously.

Definition 4.4 We now define the error control functions

$$
\begin{aligned}
& H_{1}\left(r^{*}\right):=\int_{r_{1}^{*}}^{r^{*}}\left\{\frac{1}{\left|V_{\text {main }}\right|^{\frac{1}{4}}} \frac{\mathrm{d}^{2}}{\mathrm{~d} r^{* 2}}\left(\frac{1}{\left|V_{\text {main }}\right|^{\frac{1}{4}}}\right)-\frac{V_{1}}{\left|V_{\text {main }}\right|^{\frac{1}{2}}}-\frac{5\left|V_{\text {main }}\right|^{\frac{1}{2}}}{16\left|\xi_{1}\right|^{3}}\right\} \mathrm{d} y, \\
& H_{2}\left(r^{*}\right):=\int_{r^{*}}^{r_{2}^{*}}\left\{\frac{1}{\left|V_{\text {main }}\right|^{\frac{1}{4}}} \frac{\mathrm{d}^{2}}{\mathrm{~d} r^{* 2}}\left(\frac{1}{\left|V_{\text {main }}\right|^{\frac{1}{4}}}\right)-\frac{V_{1}}{\left|V_{\text {main }}\right|^{\frac{1}{2}}}-\frac{5\left|V_{\text {main }}\right|^{\frac{1}{2}}}{16\left|\xi_{2}\right|^{3}}\right\} \mathrm{d} y
\end{aligned}
$$


which are equivalently characterized (using (4.41), see also [94, Chapter 11, $\S 3.3$, equ. (3.07), (3.08)]) as

$$
\begin{aligned}
& H_{1}\left(r^{*}\right)=-\int_{0}^{\xi_{1}\left(r^{*}\right)}|v|^{-\frac{1}{2}} \Psi_{1}(v) \mathrm{d} v, \\
& H_{2}\left(r^{*}\right)=-\int_{0}^{\xi_{2}\left(r^{*}\right)}|v|^{-\frac{1}{2}} \Psi_{2}(v) \mathrm{d} v .
\end{aligned}
$$

Lemma 4.4 The error control functions $H_{1}$ and $\mathrm{H}_{2}$ satisfy

$$
\begin{aligned}
& \mathcal{V}_{-\infty, r_{2}^{*}-\epsilon}\left(H_{1}\right) \lesssim 1, \\
& \mathcal{V}_{r_{1}^{*}+\epsilon, l \frac{\pi}{2}}\left(H_{2}\right) \lesssim 1
\end{aligned}
$$

Proof We begin with $H_{2}$. We have that $\xi_{2}\left(\left[r_{1}^{*}+\epsilon, \frac{\pi}{2} l\right]\right)$ is compact as $\xi_{2}$ is continuous. Moreover, since $\xi_{2} \mapsto \Psi_{2}$ is continuous we have $\sup _{\xi_{2}\left(\left[r_{1}^{*}+\epsilon, \frac{\pi}{2} l\right]\right)}\left|\Psi_{2}\right| \lesssim 1$. As $|v|^{-\frac{1}{2}} \in L_{l o c}^{1}$ we obtain (4.48) as

$$
\mathcal{V}_{r_{1}^{*}+\epsilon, l \frac{\pi}{2}}\left(H_{2}\right) \leq \int_{\xi_{2}\left(\frac{\pi l}{2}\right)}^{\xi_{2}\left(r_{1}^{*}+\epsilon\right)}|v|^{-\frac{1}{2}}\left|\Psi_{2}(v)\right| \mathrm{d} v \lesssim 1
$$

For $H_{1}$, we have to deal with the unbounded region $r^{*} \in\left(-\infty, r_{2}^{*}-\epsilon\right]$. We decompose

$$
\mathcal{V}_{-\infty, r_{2}^{*}-\epsilon}\left(H_{1}\right)=\mathcal{V}_{-\infty, r_{1}^{*}-1}\left(H_{1}\right)+\mathcal{V}_{r_{1}^{*}-1, r_{2}^{*}-\epsilon}\left(H_{1}\right)
$$

Completely analogous to the proof of the bound on $\mathrm{H}_{2}$ we have

$$
\mathcal{V}_{r_{1}^{*}-1, r_{2}^{*}-\epsilon}\left(H_{1}\right) \lesssim 1
$$

For the term $\mathcal{V}_{-\infty, r_{1}^{*}-1}\left(H_{1}\right)$ we remark that

$$
-V_{\text {main }} \sim 1, \quad\left|V_{\text {main }}^{\prime}\right|,\left|V_{\text {main }}^{\prime \prime}\right| \lesssim e^{2 \kappa_{+} r^{*}} \text { and }\left|V_{1}\right| \lesssim e^{2 \kappa_{+} r^{*}}
$$

for $r^{*} \in\left(-\infty, r_{1}^{*}-1\right)$. Using the lower bound $-V_{\text {main }}$, we infer from (4.32) that

$$
-\xi_{1}\left(r^{*}\right) \gtrsim\left(-r^{*}\right)^{\frac{2}{3}}
$$


for $r^{*} \in\left(-\infty, r_{1}^{*}-1\right)$. Hence,

$$
\begin{aligned}
\mathcal{V}_{-\infty, r_{1}^{*}-1}\left(H_{1}\right) \lesssim & \int_{-\infty}^{r_{1}^{*}-1}\left|\frac{V_{\text {main }}^{\prime \prime}}{\left|V_{\text {main }}\right|^{\frac{3}{2}}}\right|+\left|\frac{V_{\text {main }}^{\prime 2}}{\left|V_{\text {main }}\right|^{\frac{5}{2}}}\right| \\
& +\left|\frac{V_{1}}{\left|V_{\text {main }}\right|^{\frac{1}{2}}}\right|+\frac{\left|V_{\text {main }}\right|^{\frac{1}{2}}}{\left|\xi_{1}\right|^{3}} \mathrm{~d} r^{*} \\
& \lesssim \int_{-\infty}^{r_{1}^{*}-1} e^{2 \alpha_{+} r^{*}}+\frac{1}{\left|r^{*}\right|^{2}} \mathrm{~d} r^{*} \lesssim 1 .
\end{aligned}
$$

With the bounds in Lemma 4.4 in hand, we apply [94, Chapter 11, Theorem 3.1] which allow us to define the following.

Proposition 4.1 We define solutions to the radial o.d.e. (2.43) for $\omega=\omega_{-} m$ as

$$
\begin{aligned}
& u_{\mathrm{Ai1}}\left(r^{*}, m\right):=\hat{f}_{1}^{\frac{1}{4}}\left(r_{1}^{*}\right) \hat{f}_{1}^{-\frac{1}{4}}\left(r^{*}\right)\left\{\operatorname{Ai}\left(m^{\frac{2}{3}} \xi_{1}\right)+\epsilon_{\mathrm{Ai} 1}\left(m, r^{*}\right)\right\} \text { for } r^{*} \in\left(-\infty, r_{2}^{*}-\epsilon\right], \\
& u_{\mathrm{Bi} 1}\left(r^{*}, m\right):=\hat{f}_{1}^{\frac{1}{4}}\left(r_{1}^{*}\right) \hat{f}_{1}^{-\frac{1}{4}}\left(r^{*}\right)\left\{\operatorname{Bi}\left(m^{\frac{2}{3}} \xi_{1}\right)+\epsilon_{\mathrm{Bi} 1}\left(m, r^{*}\right)\right\} \text { for } r^{*} \in\left(-\infty, r_{2}^{*}-\epsilon\right], \\
& u_{\mathrm{Ai2} 2}\left(r^{*}, m\right):=\hat{f}_{2}^{\frac{1}{4}}\left(r_{2}^{*}\right) \hat{f}_{2}^{-\frac{1}{4}}\left(r^{*}\right)\left\{\operatorname{Ai}\left(m^{\frac{2}{3}} \xi_{2}\right)+\epsilon_{\mathrm{Ai} 2}\left(m, r^{*}\right)\right\} \text { for } r^{*} \in\left[r_{1}^{*}+\epsilon, \frac{\pi}{2} l\right], \\
& u_{\mathrm{Bi} 2}\left(r^{*}, m\right):=\hat{f}_{2}^{\frac{1}{4}}\left(r_{2}^{*}\right) \hat{f}_{2}^{-\frac{1}{4}}\left(r^{*}\right)\left\{\operatorname{Bi}\left(m^{\frac{2}{3}} \xi_{2}\right)+\epsilon_{\mathrm{Bi} 2}\left(m, r^{*}\right)\right\} \text { for } r^{*} \in\left[r_{1}^{*}+\epsilon, \frac{\pi}{2} l\right] .
\end{aligned}
$$

Moreover,

$$
\begin{aligned}
&\left|\epsilon_{\mathrm{Ai} 1}\right| \lesssim M_{\mathrm{Ai}}\left(m^{\frac{2}{3}} \xi_{1}\right) E_{\mathrm{Ai}}^{-1}\left(m^{\frac{2}{3}} \xi_{1}\right) m^{-1}, \\
&\left|\epsilon_{\mathrm{Ai} 1}^{\prime}\right| \lesssim \hat{f}_{1}^{\frac{1}{2}} N_{\mathrm{Ai}}\left(m^{\frac{2}{3}} \xi_{1}\right) E_{\mathrm{Ai}}^{-1}\left(m^{\frac{2}{3}} \xi_{1}\right) m^{-\frac{1}{3}}, \\
&\left|\epsilon_{\mathrm{Bi} 1}\right| \lesssim \epsilon_{\mathrm{Ai}}\left(m^{\frac{2}{3}} \xi_{1}\right) E_{\mathrm{Ai}}\left(m^{\frac{2}{3}} \xi_{1}\right) m^{-1}, \\
&\left|\epsilon_{\mathrm{Bi} 1}^{\prime}\right| \lesssim \hat{f}_{1}^{\frac{1}{2}} N_{\mathrm{Ai}}\left(m^{\frac{2}{3}} \xi_{1}\right) E_{\mathrm{Ai}}\left(m^{\frac{2}{3}} \xi_{1}\right) m^{-\frac{1}{3}}, \\
&\left|\epsilon_{\mathrm{Ai} 2}\right| \lesssim M_{\mathrm{Ai}}\left(m^{\frac{2}{3}} \xi_{2}\right) E_{\mathrm{Ai}}^{-1}\left(m^{\frac{2}{3}} \xi_{2}\right) m^{-1}, \\
&\left|\epsilon_{\mathrm{Ai} 2}^{\prime}\right| \lesssim \hat{f}_{2}^{\frac{1}{2}} N_{\mathrm{Ai}}\left(m^{\frac{2}{3}} \xi_{2}\right) E_{\mathrm{Ai}}^{-1}\left(m^{\frac{2}{3}} \xi_{2}\right) m^{-\frac{1}{3}}, \\
&\left|\epsilon_{\mathrm{Bi} 2}\right| \lesssim M_{\mathrm{Ai}}\left(m^{\frac{2}{3}} \xi_{2}\right) E_{\mathrm{Ai}}\left(m^{\frac{2}{3}} \xi_{2}\right) m^{-1}, \\
&\left|\epsilon_{\mathrm{Bi} 2}^{\prime}\right| \lesssim \hat{f}_{2}^{\frac{1}{2}} N_{\mathrm{Ai}}\left(m^{\frac{2}{3}} \xi_{2}\right) E_{\mathrm{Ai}}\left(m^{\frac{2}{3}} \xi_{2}\right) m^{-\frac{1}{3}},
\end{aligned}
$$

where (4.59)-(4.62) hold uniformly in $r^{*} \in\left(-\infty, r_{2}^{*}-\epsilon\right]$ and (4.63)-(4.66) hold uniformly in $r^{*} \in\left[r_{1}^{*}+\epsilon, \frac{\pi}{2} l\right]$. Further, we choose the initialization such that 


$$
\begin{aligned}
& \left|\epsilon_{\mathrm{Ai} 2}\left(r^{*}\right)\right| \lesssim M_{\mathrm{Ai}}\left(m^{\frac{2}{3}} \xi_{2}\right) E_{\mathrm{Ai}}^{-1}\left(m^{\frac{2}{3}} \xi_{2}\right)\left(\exp \left[2 \mathcal{V}_{r_{1}^{*}+\epsilon, r^{*}}\left(H_{2}\right) m^{-1}\right]-1\right) \\
& \left|\epsilon_{\mathrm{Bi} 2}\left(r^{*}\right)\right| \lesssim M_{\mathrm{Ai}}\left(m^{\frac{2}{3}} \xi_{2}\right) E_{\mathrm{Ai}}\left(m^{\frac{2}{3}} \xi_{2}\right)\left(\exp \left[2 \mathcal{V}_{r^{*}, l \frac{\pi}{2}}\left(H_{2}\right) m^{-1}\right]-1\right)
\end{aligned}
$$

and in particular, $\left|\epsilon_{\mathrm{Ai} 2}\left(\frac{\pi}{2} l\right)\right| \lesssim m^{-\frac{7}{6}}, \epsilon_{\mathrm{Bi} 2}\left(\frac{\pi}{2} l\right)=0$.

Proof The proof follows from [91, Chapter 11, Theorem 3.1]. For convenience of the reader we will outline the proof for $u_{\mathrm{Ai} 2}$. Indeed, with the ansatz of (4.57) we note that from (4.38), we have $W_{2}=\hat{f}_{2}^{\frac{1}{4}}\left(r_{2}^{*}\right)\left\{\operatorname{Ai}\left(m^{\frac{2}{3}} \xi_{2}\right)+\epsilon_{\mathrm{Ai} 2}\left(\xi_{2}\right)\right\}$. Thus, from (4.38) and variation of parameters (see [94, Chapter 11, equ. (3.12)] or [92]), the error $\epsilon_{\mathrm{Ai} 2}\left(\xi_{2}\right)$ solves

$$
\epsilon_{\mathrm{Ai} 2}\left(\xi_{2}\right)=-\pi m^{-\frac{2}{3}} \int_{\xi_{2}}^{\xi_{2}\left(r_{1}^{*}+\epsilon\right)} K\left(\xi_{2}, v\right) \Psi_{2}(v)\left[\epsilon_{\mathrm{Ai} 2}(v)+\operatorname{Ai}\left(m^{\frac{2}{3}} v\right)\right] \mathrm{d} v,
$$

where $K\left(\xi_{2}, v\right)=\operatorname{Bi}\left(m^{\frac{2}{3}} \xi_{2}\right) \operatorname{Ai}\left(m^{\frac{2}{3}} v\right)-\operatorname{Bi}\left(m^{\frac{2}{3}} v\right) \operatorname{Ai}\left(m^{\frac{2}{3}} \xi_{2}\right)$. We note that as in [94, Chapter 11, equ. (3.12)], the kernel $K$ satisfies

$$
\left|K\left(\xi_{2}, v\right)\right| \leq E_{\mathrm{Ai}}^{-1}\left(m^{\frac{2}{3}} \xi_{2}\right) E_{\mathrm{Ai}}\left(m^{\frac{2}{3}} v\right) M_{\mathrm{Ai}}\left(m^{\frac{2}{3}} \xi_{2}\right) M_{\mathrm{Ai}}\left(m^{\frac{2}{3}} v\right)
$$

for $v \geq \xi_{2}$ and similarly,

$$
\left|\partial_{\xi_{2}} K\left(\xi_{2}, v\right)\right| \leq m^{\frac{2}{3}} E_{\mathrm{Ai}}^{-1}\left(m^{\frac{2}{3}} \xi_{2}\right) E_{\mathrm{Ai}}\left(m^{\frac{2}{3}} v\right) N_{\mathrm{Ai}}\left(m^{\frac{2}{3}} \xi_{2}\right) M_{\mathrm{Ai}}\left(m^{\frac{2}{3}} v\right)
$$

for $v \geq \xi_{2}$. Now, as in [94, Chapter 11, equ. (3.12)], the integral equation (4.69) is solved using [94, Chapter 6, Theorem 10.2] which shows the bounds together with Lemma 4.4.

\section{The non-Diophantine condition}

With the fundamental solutions from Sect. 4.2, we are now in the position to define the set of black hole parameters $\mathscr{P}_{\text {Blow-up }}$. The set $\mathscr{P}_{\text {Blow-up will be }}$ defined in Definition 5.3 as a suitable lim sup set which constitutes a (generalized) non-Diophantine condition.

\subsection{Definition of the non-Diophantine condition as the set $\mathscr{P}_{\text {Blow-up }}$}

We first define Wronskians of solutions of the radial o.d.e. which will play a fundamental role in the estimates. 
Definition 5.1 We define $\mathfrak{W}_{1}: \mathscr{P} \times \mathbb{Z}_{m} \times \mathbb{Z}_{\ell \geq|m|} \rightarrow \mathbb{C}$ and $\mathfrak{W}_{2}: \mathscr{P} \times \mathbb{Z}_{m} \times$ $\mathbb{Z}_{\ell \geq|m|} \cap\left\{\sigma_{1} \leq \lambda_{m \ell} m^{-2} \leq \sigma_{2}\right\} \rightarrow \mathbb{C}$ as

$$
\begin{aligned}
& \mathfrak{W}_{1}(\mathfrak{p}, m, \ell):=\mathfrak{W}\left[u_{\mathcal{H}^{+}}, u_{\infty}\right]\left(m, \ell, \omega=\omega_{-} m, \mathfrak{p}\right), \\
& \mathfrak{W}_{2}(\mathfrak{p}, m, \ell):=\mathfrak{W}\left[u_{\mathrm{Ai} 2}, u_{\infty}\right]\left(m, \ell, \omega=\omega_{-} m, \mathfrak{p}\right) .
\end{aligned}
$$

Note that this is well-defined as the Wronskians $\mathfrak{W}_{1}$ and $\mathfrak{W}_{2}$ only depend on $\mathscr{P}$ (by construction). Moreover, by continuous dependence on parameters of solutions to o.d.e.'s, the Wronskians $\mathfrak{W}_{1}$ and $\mathfrak{W}_{2}$ are continuous functions on $\mathscr{P}$ for fixed $m, \ell$.

Remark 5.1 Note that, as discussed in the introduction, the Wronskian $\mathfrak{W}_{1}$ does not vanish. Nevertheless, $\mathfrak{W}_{1}$ can be very small (as $m, \ell \rightarrow \infty$ ) which corresponds to frequency parameters associated to stable trapping. On the other hand, $\mathfrak{W}_{2}$ may vanish and this indeed corresponds to stable trapping. In particular, if $\mathfrak{W}_{2}$ vanishes, then the solution $u_{\infty}$ is a multiple of the $u_{\mathrm{Ai} 2}$ which is exponentially damped in the semi-classical forbidden region. In this case, we infer that $\mathfrak{W}_{1}$ is exponentially small and indeed, we are in the situation of stable trapping. This would then show that there exists a quasimode with frequency $\omega=\omega_{-} m$. This intuition leads to the following non-Diophantine condition for the set $\mathscr{P}_{\text {Blow-up. }}$

Definition 5.2 For $m_{0} \in \mathbb{N}$ we define

$$
U_{m_{0}}:=\bigcup_{\substack{m \geq m_{0} \\ m \in \mathbb{N}}} \bigcup_{\substack{m \leq \ell \leq m^{2} \\ \ell \in \mathbb{N}}} U(m, \ell),
$$

where

$$
\begin{aligned}
U(m, \ell):= & \left\{\mathfrak{p} \in \mathscr{P}:\left|\mathfrak{W}_{1}(\mathfrak{p}, m, \ell)\right|<e^{-\sqrt{m}}, \sigma_{1}(\mathfrak{p})<\lambda_{m \ell}\left(a \omega_{-}(\mathfrak{p}) m\right)<\sigma_{2}(\mathfrak{p}),\right. \\
& \left|\mathfrak{W}_{2}(\mathfrak{p}, m, \ell)\right|<e^{-\ell} e^{-m},\left|\Gamma \mathfrak{W}_{2}(\mathfrak{p}, m, \ell)\right|>1 \\
& \left.\left|\mathfrak{W}_{2}\left(\Phi_{\tau}^{\Gamma}(\mathfrak{p}), m, \ell\right)\right|>e^{-\ell} e^{-m} \text { for all }|\tau| \in\left[e^{-\ell} e^{-m}, \frac{1}{m^{2}}\right]\right\} .
\end{aligned}
$$

Definition 5.3 We define

$$
\mathscr{P}_{\text {Blow-up }}:=\bigcap_{m_{0} \in \mathbb{N}} U_{m_{0}} .
$$

While a priori the set $\mathscr{P}_{\text {Blow-up }}$ could be empty, we will show in the following that it is dense in $\mathscr{P}$ and Baire-generic, i.e. a countable intersection of open and dense sets. 


\subsection{Topological genericity: $\mathscr{P}_{\text {Blow-up is Baire-generic }}$}

We will first show that each $U_{m_{0}}$ is dense. To do so, we let $m_{0}$ and $\mathfrak{p}_{0}=$ $\left(\mathfrak{m}_{0}, \mathfrak{a}_{0}\right) \in \mathscr{P}$ be arbitrary and fixed throughout Sect. 5.2. Also, let $\mathcal{U} \subset \mathscr{P}$ be an open neighborhood of $\mathfrak{p}_{0}$. We will show that there exists an element of $U_{m_{0}}$ which is contained in $\mathcal{U}$. We now define a curve of parameters through $\mathfrak{p}_{0}$ as follows.

Definition 5.4 For $\delta=\delta\left(\mathfrak{p}_{0}, \mathcal{U}\right)>0$ sufficiently small, we define the smooth embedded curve $\gamma_{\delta}\left(\mathfrak{p}_{0}\right) \subset \mathcal{U}$ through $\mathfrak{p}_{0}$ as

$$
\gamma_{\delta}\left(\mathfrak{p}_{0}\right):=\left\{\mathfrak{p}=(\mathfrak{m}, \mathfrak{a}) \in \mathscr{P}: \mathfrak{a}=\mathfrak{a}_{0},\left|\vartheta(\mathfrak{p})-\vartheta\left(\mathfrak{p}_{0}\right)\right| \leq \delta\right\}
$$

Throughout Sect. 5.2 we will only consider

$$
\mathfrak{p} \in \gamma_{\delta}\left(\mathfrak{p}_{0}\right)
$$

We parameterize $\gamma_{\delta}\left(\mathfrak{p}_{0}\right)$ with $\vartheta \in\left(\vartheta_{0}-\delta, \vartheta_{0}+\delta\right)$, where $\vartheta_{0}=\vartheta\left(\mathfrak{p}_{0}\right)$.

Remark 5.2 Note that the expression $\Xi$ is (by construction) constant on $\gamma_{\delta}\left(\mathfrak{p}_{0}\right)$. We also note that $\gamma_{\delta}\left(\mathfrak{p}_{0}\right)$ can be seen as a variation of $\mathfrak{m}$ keeping $\mathfrak{a}$ fixed. It is however more convention to use the coordinates $(\vartheta, \mathfrak{a})$ as they are adapted to the interior scattering pole in view of $\vartheta=a \omega_{-}$.

Lemma 5.1 The angular eigenvalues at the interior scattering poles $\omega=$ $\omega \_m$ satisfy

$$
\Gamma\left(\lambda_{m \ell}\left(a \omega_{-} m\right)+a^{2} \omega_{-}^{2} m^{2}-2 a \omega_{-} m^{2} \Xi\right) \in\left[-4 m^{2}, 0\right] .
$$

Proof Note that

$$
\lambda_{m \ell}\left(a \omega \_m\right)+a^{2} \omega_{-}^{2} m^{2}-2 a \omega \_m^{2} \Xi
$$

is an eigenvalue of the operator

$$
\begin{aligned}
P_{\omega_{-}} & +a^{2} \omega_{-}^{2} m^{2}-2 a \omega_{-} m^{2} \Xi \\
= & -\frac{\mathrm{d}}{\mathrm{d} x}\left(\Delta_{x}\left(1-x^{2}\right) \frac{\mathrm{d}}{\mathrm{d} x} \cdot\right)+\frac{m^{2}}{\Delta_{x}}\left(\frac{\Xi}{\sqrt{1-x^{2}}}-a \omega_{-} \sqrt{1-x^{2}}\right)^{2} \\
& +2 \frac{a^{2}}{l^{2}}\left(1-x^{2}\right) .
\end{aligned}
$$


Now, by construction of $\Gamma$ in (2.11) we have $\Gamma\left(a \omega_{-}\right)=\Gamma(\vartheta)=1$ and $\Gamma(a)=0$ to compute

$$
\begin{aligned}
\Gamma & \left(P_{\omega_{-}}+a^{2} \omega_{-}^{2} m^{2}-2 a \omega_{-} m^{2} \Xi\right) \\
& =-2 \frac{m^{2}}{\Delta_{x}}\left(\Xi-a \omega_{-}\left(1-x^{2}\right)\right)+2 a \omega_{-} m^{2}-2 m^{2} \Xi \\
& =-2 m^{2} \frac{\Xi}{\Delta_{x}} \frac{r_{-}^{2}+a^{2}-a^{2}+a^{2} x^{2}-a^{2} \Delta_{x}+\left(r_{-}^{2}+a^{2}\right) \Delta_{x}}{r_{-}^{2}+a^{2}} \\
& =-2 m^{2} \frac{\Xi}{\Delta_{x}}\left(1-\frac{r_{-}^{2}-a^{2}}{r_{-}^{2}+a^{2}} \Delta_{x}\right) \in\left[-4 m^{2}, 0\right]
\end{aligned}
$$

as $\left|\frac{r_{-}^{2}-a^{2}}{r_{-}^{2}+a^{2}} \Delta_{x}\right| \leq 1$ and $0 \leq \frac{\Xi}{\Delta_{x}} \leq 1$.

Lemma 5.2 We have $\left|\Gamma\left(\epsilon_{\mathrm{Ai} 2}\left(\frac{\pi}{2} l\right)\right)\right| \lesssim m^{-\frac{1}{6}}$ where $\epsilon_{\mathrm{Ai} 2}$ is defined in Proposition 4.1 .

Proof First, we consider $\epsilon_{\mathrm{Ai} 2}$ as a function of $\xi_{2}$ defined in Definition 4.3. Then, applying $\Gamma$ to (4.69) shows that $\Gamma \epsilon_{\mathrm{Ai} 2}$ solves

$$
\begin{aligned}
\Gamma \epsilon_{\mathrm{Ai} 2}\left(\xi_{2}\right)= & -\pi m^{-\frac{2}{3}} \int_{\xi_{2}}^{\xi_{2}\left(r_{1}^{*}+\epsilon\right)} K\left(\xi_{2}, v\right) \Psi_{2}(v) \Gamma \epsilon_{\mathrm{Ai} 2}(v) \mathrm{d} v \\
& -\pi m^{-\frac{2}{3}} \int_{\xi_{2}}^{\xi_{2}\left(r_{1}^{*}+\epsilon\right)} K\left(\xi_{2}, v\right) \Gamma\left(\Psi_{2}(v)\right) \\
& \times\left[\epsilon_{\mathrm{Ai} 2}(v)+\operatorname{Ai}\left(m^{\frac{2}{3}} v\right)\right] \mathrm{d} v \\
& -\Gamma\left(\xi_{2}\left(r_{1}^{*}+\epsilon\right)\right) \pi m^{-\frac{2}{3}} K\left(\xi_{2}, \xi_{2}\left(r_{1}^{*}+\epsilon\right)\right) \\
& \times \Psi_{2}\left(\xi_{2}\left(r_{1}^{*}+\epsilon\right)\right) \operatorname{Ai}\left(m^{\frac{2}{3}} \xi_{2}\left(r_{1}^{*}+\epsilon\right)\right) .
\end{aligned}
$$

We denote $k\left(\xi_{2}\right):=-\Gamma\left(\xi_{2}\left(r_{1}^{*}+\epsilon\right)\right) \pi m^{-\frac{2}{3}} K\left(\xi_{2}, \xi_{2}\left(r_{1}^{*}+\epsilon\right)\right) \Psi_{2}\left(\xi_{2}\left(r_{1}^{*}+\right.\right.$ $\epsilon) \operatorname{Ai}\left(m^{\frac{2}{3}} \xi_{2}\left(r_{1}^{*}+\epsilon\right)\right)$ which satisfies

$$
\left|k\left(\xi_{2}\right)\right| \lesssim m^{-\frac{2}{3}} M_{\mathrm{Ai}}\left(m^{\frac{2}{3}} \xi_{2}\right) M_{\mathrm{Ai}}^{2}\left(m^{\frac{2}{3}} \xi_{2}\left(r_{1}^{*}+\epsilon\right)\right) E_{\mathrm{Ai}}^{-1}\left(m^{\frac{2}{3}} \xi_{2}\right),
$$

where we used that $\left|\Gamma\left(\xi_{2}\left(r_{1}^{*}+\epsilon\right)\right)\right| \lesssim 1$ and $\left|\Psi_{2}\left(\xi_{2}\left(r_{1}^{*}+\epsilon\right)\right)\right| \lesssim 1$. Now, we re-write the equation for the unknown $\left(\Gamma \epsilon_{\mathrm{Ai} 2}-k\right)\left(\xi_{2}\right)$ as 


$$
\begin{aligned}
& \left(\Gamma \epsilon_{\mathrm{Ai} 2}-k\right)\left(\xi_{2}\right)=-\pi m^{-\frac{2}{3}} \int_{\xi_{2}}^{\xi_{2}\left(r_{1}^{*}+\epsilon\right)} K\left(\xi_{2}, v\right) \Psi_{2}(v)\left(\Gamma \epsilon_{\mathrm{Ai} 2}-k\right)(v) \mathrm{d} v \\
& -\pi m^{-\frac{2}{3}} \int_{\xi_{2}}^{\xi_{2}\left(r_{1}^{*}+\epsilon\right)} K\left(\xi_{2}, v\right) \\
& \quad \times\left[\Gamma\left(\Psi_{2}(v)\right)\left(\epsilon_{\mathrm{Ai} 2}(v)+\operatorname{Ai}\left(m^{\frac{2}{3}} v\right)\right)+\Psi_{2}(v) k(v)\right] \mathrm{d} v
\end{aligned}
$$

First, from (4.3) we know that $\Psi_{2}$ depends smoothly on $\mathfrak{p}$ and in particular, that $\xi_{2} \mapsto \Gamma \Psi_{2}\left(\xi_{2}\right)$ is smooth. Since $\left|l^{2} \Gamma V_{\text {main }}\right| \lesssim 1$ (using Lemma 5.1) and $\left|l^{2} \Gamma V_{1}\right| \lesssim 1$, this is made quantitative to obtain $\left|\Gamma \Psi_{2}\right| \lesssim 1$ uniformly in the compact set $\xi_{2}\left[r_{1}^{*}+\epsilon, l \frac{\pi}{2}\right]$.

Now, we apply [94, Theorem 10.1, Chapter 6] and in the notation of [94, Theorem 10.1, Chapter 6] we set

$$
\begin{aligned}
& \mathrm{K}\left(\xi_{2}, v\right)=\pi m^{-\frac{2}{3}} K\left(\xi_{2}, v\right)|v|^{\frac{1}{2}}, \\
& P_{0}\left(\xi_{2}\right)=E_{\mathrm{Ai}}^{-1}\left(m^{\frac{2}{3}} \xi_{2}\right) M_{\mathrm{Ai}}\left(m^{\frac{2}{3}} \xi_{2}\right), \\
& P_{1}\left(\xi_{2}\right)=m^{\frac{2}{3}} E_{\mathrm{Ai}}^{-1}\left(m^{\frac{2}{3}} \xi_{2}\right) N_{\mathrm{Ai}}\left(m^{\frac{2}{3}} \xi_{2}\right), \\
& Q(v)=\pi m^{-\frac{2}{3}}|v|^{\frac{1}{2}} E_{\mathrm{Ai}}\left(m^{\frac{2}{3}} v\right) M_{\mathrm{Ai}}\left(m^{\frac{2}{3}} v\right), \\
& \psi_{0}(v)=|v|^{-\frac{1}{2}} \Psi_{2}(v), \\
& \phi(v)=|v|^{-\frac{1}{2}}, \\
& \psi_{1}(v)=0, \\
& J(v)=\Gamma\left(\Psi_{2}(v)\right)\left(\epsilon_{\mathrm{Ai} 2}(v)+\operatorname{Ai}\left(m^{\frac{2}{3}} v\right)\right)+\Psi_{2}(v) k(v) .
\end{aligned}
$$

We further have that

$$
\begin{aligned}
& \Phi\left(\xi_{2}\right):=\int_{\xi_{2}}^{\xi_{2}\left(r_{1}^{*}+\epsilon\right)}|\phi(v)| \mathrm{d} v=\int_{\xi_{2}}^{\xi_{2}\left(r_{1}^{*}+\epsilon\right)}|v|^{-\frac{1}{2}} \mathrm{~d} v, \\
& \Psi_{0}\left(\xi_{2}\right):=\int_{\xi_{2}}^{\xi_{2}\left(r_{1}^{*}+\epsilon\right)}\left|\psi_{0}(v)\right| \mathrm{d} v=\int_{\xi_{2}}^{\xi_{2}\left(r_{1}^{*}+\epsilon\right)}|v|^{-\frac{1}{2}}\left|\Psi_{2}(v)\right| \mathrm{d} v
\end{aligned}
$$

exist and satisfy $\sup _{v \in \xi_{2}\left(r_{1}^{*}+\epsilon, l \frac{\pi}{2}\right)} \Phi(v) \lesssim 1$, as well as $\sup _{v \in \xi_{2}\left(r_{1}^{*}+\epsilon, l \frac{\pi}{2}\right)} \Psi_{0}(v) \lesssim$ 1 as in (4.49) of Lemma 4.4. With the above choices, Lemma 4.4 and (4.70), (4.71), we have that assumptions (i)-(vi) of [94, Theorem 10.1, Chapter 6] are satisfied.

Now, we compute $\kappa:=\sup _{v \in \xi_{2}\left[r_{1}^{*}+\epsilon, l \frac{\pi}{2}\right]} Q(v)|J(v)|$, for which we note that the largest term in $J$ is coming from $\Gamma\left(\Psi_{2}\right) \operatorname{Ai}\left(m^{\frac{2}{3}} v\right)$ in view of the shown properties for $\epsilon_{\mathrm{Ai} 2}$ from (4.63) and $k(v)$ from (5.13). Since 
$\left|\Gamma\left(\Psi_{2}\right)(v) \operatorname{Ai}\left(m^{\frac{2}{3}} v\right)\right| \lesssim M_{\mathrm{Ai}}\left(m^{\frac{2}{3}} v\right) E_{\mathrm{Ai}}^{-1}\left(m^{\frac{2}{3}} v\right)$, we obtain $\kappa \lesssim m^{-\frac{2}{3}}$. Further, for $\kappa_{0}:=\sup _{v \in \xi_{2}\left[r_{1}^{*}+\epsilon, l \frac{\pi}{2}\right]} Q(v)\left|P_{0}(v)\right|$ we directly obtain the estimate $\kappa_{0} \lesssim m^{-\frac{2}{3}}$.

Thus, the assumptions of [94, Theorem 10.1, Chapter 6] are satisfied and we obtain that $\Gamma \epsilon_{\mathrm{Ai} 2}-k$ satisfies

$$
\begin{aligned}
\sup _{\xi_{2} \in \xi_{2}\left(r_{1}^{*}+\epsilon, l \frac{\pi}{2}\right)}\left|\Gamma \epsilon_{\mathrm{Ai} 2}-k\right|\left(\xi_{2}\right) & \leq \sup _{\xi_{2} \in \xi_{2}\left(r_{1}^{*}+\epsilon, l \frac{\pi}{2}\right)} P_{0}\left(\xi_{2}\right) \kappa \Phi\left(\xi_{2}\right) \exp \left(\kappa_{0} \Psi_{0}\left(\xi_{2}\right)\right) \\
& \lesssim m^{-\frac{2}{3}}
\end{aligned}
$$

Thus,

$$
\left|\left(\Gamma \epsilon_{\mathrm{Ai} 2}\right)\left(\xi_{2}(\pi l / 2)\right)\right| \lesssim m^{-\frac{2}{3}}+|k(\pi l / 2)| \lesssim m^{-\frac{2}{3}}
$$

in view of (5.13). Finally,

$$
\begin{aligned}
\Gamma\left(\epsilon_{\mathrm{Ai} 2}\left(\xi_{2}(\pi l / 2)\right)\right) & \leq\left|\left(\Gamma \epsilon_{\mathrm{Ai} 2}\right)\left(\xi_{2}(\pi l / 2)\right)\right|+\left|\frac{\mathrm{d} \epsilon_{\mathrm{Ai} 2}}{\mathrm{~d} \xi_{2}}\left(\xi_{2}(l \pi / 2)\right) \Gamma\left(\xi_{2}(l \pi / 2)\right)\right| \\
& \lesssim m^{-\frac{2}{3}}+\left|\frac{\mathrm{d} \epsilon_{\mathrm{Ai} 2}}{\mathrm{~d} r^{*}}(l \pi / 2)\left(\frac{\mathrm{d} \xi_{2}}{\mathrm{~d} r^{*}}\right)^{-1}(l \pi / 2) \Gamma\left(\xi_{2}(l \pi / 2)\right)\right| \\
& \lesssim m^{-\frac{2}{3}}+m^{-\frac{1}{3}} N_{\mathrm{Ai}}\left(m^{\frac{2}{3}} \xi_{2}(l \pi / 2)\right) \\
& \lesssim m^{-\frac{2}{3}}+m^{-\frac{1}{3}} m^{\frac{2}{3} \cdot \frac{1}{4}} \xi_{2}(l \pi / 2)^{\frac{1}{4}} \lesssim m^{-\frac{1}{6}}
\end{aligned}
$$

which concludes the proof.

Remark 5.3 From Proposition 3.2 and Lemma 5.1 we have that for almost every $\tilde{\lambda}_{0}>\Xi^{2}$, there exist sequences $\left(m_{i}\right)_{i \in \mathbb{N}}$ and $\left(\ell_{i}\right)_{i \in \mathbb{N}}\left(m_{i} \leq \ell_{i} \leq m_{i}^{2}\right)$ with $m_{i} \rightarrow \infty, \ell_{i} \rightarrow \infty$ as $i \rightarrow \infty$ such that the angular eigenvalues satisfy

$$
\lambda_{i}=\lambda_{m_{i} \ell_{i}}\left(\omega=\omega_{-} m_{i}\right)=\tilde{\lambda}_{i} m_{i}^{2}=\tilde{\lambda}_{0} m_{i}^{2}+\lambda_{\text {error }}^{(i)},
$$

where $\left|\lambda_{\text {error }}^{(i)}\left(\mathfrak{p}_{0}\right)\right| \leq C\left(\tilde{\lambda}_{0}, \mathfrak{p}_{0}\right)$ and $\left|\lambda_{\text {error }}^{(i)}(\mathfrak{p})\right| \leq C\left(\tilde{\lambda}_{0}, \mathfrak{p}_{0}\right)\left(1+\left|\vartheta_{0}-\vartheta\right| m_{i}^{2}\right) \lesssim$ $1+\delta m_{i}^{2}$ uniformly for $\mathfrak{p} \in \gamma_{\delta}\left(\mathfrak{p}_{0}\right)$ as $m_{i} \rightarrow \infty$. Moreover, we assume without loss of generality that $m_{i+1}>m_{i}$ and note that the choice of the subsequences $m_{i}, \ell_{i}$ depends on $\mathfrak{p}_{0}$.

Lemma 5.3 Let $\lambda_{1}:=\sup _{\mathfrak{p} \in \gamma_{\delta}\left(\mathfrak{p}_{0}\right)} \sigma_{1}(\mathfrak{p})$ and $\lambda_{2}:=\inf _{\mathfrak{p} \in \gamma_{\delta}\left(\mathfrak{p}_{0}\right)} \sigma_{2}(\mathfrak{p})$ and choose $\delta>0$ potentially smaller such that $\lambda_{1}<\lambda_{2}$. Let $\tilde{\lambda}_{0} \in\left(\lambda_{1}, \lambda_{2}\right) \backslash \mathcal{N}_{\mathfrak{p}_{0}}$ (see Remark 5.3) be arbitrary. Let $\tilde{\lambda}_{i}=\tilde{\lambda}_{0}+\lambda_{\text {error }}^{(i)} m_{i}^{-2}$ be the associated 
angular eigenvalues from Proposition 3.2 such that from (4.1) we have the associated potential $V_{\text {main }}=\frac{\Delta}{\left(r^{2}+a^{2}\right)^{2}}\left(\tilde{\lambda}_{i}+\omega_{-}^{2} a^{2}-2 a \omega_{-} \Xi\right)-\left(\omega_{-}-\omega_{r}\right)^{2}$.

Then, for all $\mathfrak{p} \in \gamma_{\delta}\left(\mathfrak{p}_{0}\right)$, and for all $i \in \mathbb{N}$ sufficiently large, we have

$$
c\left(\delta, \mathfrak{p}_{0}\right) \leq\left|\Gamma \xi_{\infty}^{(i)}\right| \leq C\left(\delta, \mathfrak{p}_{0}\right),
$$

where $\xi_{\infty}^{(i)}: \gamma_{\delta}\left(\mathfrak{p}_{0}\right) \rightarrow \mathbb{R}$ is defined as

$$
\xi_{\infty}^{(i)}:=\int_{r_{2}^{*}}^{\frac{\pi}{2} l} \sqrt{\left|V_{\text {main }}\right|} \mathrm{d} r^{*}=\int_{r_{2}}^{\infty} \sqrt{-\frac{\left(r^{2}+a^{2}\right)^{2}}{\Delta^{2}} V_{\text {main }}} \mathrm{d} r
$$

and $c\left(\delta, \mathfrak{p}_{0}\right), C\left(\delta, \mathfrak{p}_{0}\right)>0$ only depend on $\delta>0$ and $\mathfrak{p}_{0}$.

Proof We use the product rule to compute for $r \geq r_{2}$

$$
\begin{aligned}
\Gamma\left(\frac{\left(r^{2}+a^{2}\right)^{2}}{\Delta^{2}} V_{\text {main }}\right)= & \Delta^{-1} \Gamma\left(\tilde{\lambda}_{i}+\omega_{-}^{2} a^{2}-2 a \omega_{-} \Xi\right) \\
& +\Gamma\left(\Delta^{-1}\right)\left(\tilde{\lambda}_{i}+\omega_{-}^{2} a^{2}-2 a \omega_{-} \Xi\right) \\
& -\Gamma\left(\frac{\left(r^{2}+a^{2}\right)^{2}}{\Delta^{2}}\left(\omega_{-}-\omega_{r}\right)^{2}\right) .
\end{aligned}
$$

From Lemma 5.1 we know that $\Delta^{-1} \Gamma\left(\tilde{\lambda}_{i}+\omega_{-}^{2} a^{2}-2 a \omega_{-} \Xi\right) \leq 0$. Moreover, by choice of $\tilde{\lambda}_{0}$ in the assumptions of Lemma 5.3, we have that $\tilde{\lambda}_{i} \in E_{\mathfrak{p}}$ for all $\mathfrak{p} \in \gamma_{\delta}\left(\mathfrak{p}_{0}\right)$ and for all $i$ sufficiently large. Thus, using the definition of $E_{\mathfrak{p}}$ in (4.7) we have

$$
\Gamma\left(\Delta^{-1}\right)\left(\tilde{\lambda}_{i}+\omega_{-}^{2} a^{2}-2 a \omega_{-} \Xi\right)-\Gamma\left(\frac{\left(r^{2}+a^{2}\right)^{2}}{\Delta^{2}}\left(\omega_{-}-\omega_{r}\right)^{2}\right)<-\frac{\omega_{-} l^{2}}{a r^{4}}
$$

for all $r \geq r_{2}$, all $i$ sufficiently large and all $\mathfrak{p} \in \gamma_{\delta}\left(\mathfrak{p}_{0}\right)$.

Hence,

$$
\Gamma\left(\frac{\left(r^{2}+a^{2}\right)^{2}}{\Delta^{2}} V_{\text {main }}\right) \leq-\frac{\omega_{-} l^{4}}{a r^{4}}
$$

which shows

$$
\left|\Gamma \xi_{\infty}^{(i)}\right|>c\left(\delta, \mathfrak{p}_{0}\right)
$$

for all $i$ sufficiently large and for all parameters in $\gamma_{\delta}\left(\mathfrak{p}_{0}\right)$ by choosing $\delta>0$ sufficiently small. For the upper bound we also use Lemma 5.1 
to obtain that $\mid \Gamma \sqrt{\left|V_{\text {main }}\right|}=\frac{\left|\Gamma V_{\text {main }}\right|}{2\left|V_{\text {main }}^{\frac{1}{2}}\right|} \lesssim \frac{1}{\sqrt{\left|r^{*}-r_{2}^{*}\right|}}$ uniformly in $i$. The last implicit constant can also be chosen to be uniform on $\gamma_{\delta}\left(\mathfrak{p}_{0}\right)$ such that $\left|\Gamma \xi_{\infty}^{(i)}\right| \leq \int_{r_{2}^{*}}^{\frac{\pi}{2} l}\left|\Gamma \sqrt{\left|V_{\text {main }}\right|}\right| \mathrm{d} r^{*} \leq C\left(\delta, \mathfrak{p}_{0}\right)$ follows.

Now, recall the definition of $\mathfrak{W}_{1}$ and $\mathfrak{W}_{2}$ from Definition 5.1 .

Proposition 5.1 Let $m_{0} \in \mathbb{N}$. Then, there exist a parameter

$$
\mathfrak{p}_{\text {Blow-up }} \in \gamma_{\delta}\left(\mathfrak{p}_{0}\right) \subset \mathcal{U}
$$

and an $i \in \mathbb{N}$ such that $m_{0} \leq m_{i} \leq \ell_{i} \leq m_{i}^{2}$ with

$$
\begin{aligned}
& \sigma_{1}\left(\mathfrak{p}_{\text {Blow-up }}\right)<\lambda_{m_{i} \ell_{i}}\left(\omega=\omega \_m_{i}\right)<\sigma_{2}\left(\mathfrak{p}_{\text {Blow-up }}\right), \\
& \left|\mathfrak{W}_{1}\left(\vartheta_{\text {Blow-up }}\right)\right|<e^{-\sqrt{m}_{i}}
\end{aligned}
$$

as well as

$$
\begin{aligned}
& \left|\mathfrak{W}_{2}\left(\vartheta_{\text {Blow-up }}\right)\right|=0 \text { and }\left|\Gamma \mathfrak{W}_{2}\left(\vartheta_{\text {Blow-up }}\right)\right|>1 \\
& \left|\mathfrak{W}_{2}(\vartheta)\right|>e^{-\ell_{i}} e^{-m_{i}} \text { for all } e^{-\ell_{i}} e^{-m_{i}}<\left|\vartheta_{\text {Blow-up }}-\vartheta\right|<\frac{1}{m_{i}^{2}}
\end{aligned}
$$

The proof of Proposition 5.1 relies on the following two lemmata and will be given thereafter. First, we will start by showing that for every $m_{i} \geq m_{0}$ sufficiently large, there exists a $\mathfrak{p}_{\text {Blow-up }} \in \gamma_{\delta}\left(\mathfrak{p}_{0}\right)$ such that $\mathfrak{W}_{2}=0$ and $\left|\Gamma \mathfrak{W}_{2}\right|>1$. We will state this as the following lemma.

Lemma 5.4 For every $\tilde{m}_{0}>0$ there exists an $i \in \mathbb{N}$ with $m_{i}>\tilde{m}_{0}$ and a parameter $\vartheta_{\text {Blow-up with }}\left|\vartheta_{\text {Blow-up }}-\vartheta\left(\mathfrak{p}_{0}\right)\right| \leq \delta$ such that

1. $\mathfrak{W}_{2}\left(\vartheta_{\text {Blow-up }}, m_{i}\right)=0$,

2. $u_{\mathrm{Ai} 2}=\alpha_{\infty} \hat{f}_{2}^{\frac{1}{2}}\left(\frac{\pi}{2} l\right) u_{\infty}$ for $\vartheta=\vartheta_{\text {Blow-up }}$ with $\left|\alpha_{\infty}\left(\vartheta_{\text {Blow-up }}\right)\right| \sim m_{i}^{\frac{5}{6}}$,

3. $\left|\Gamma \mathfrak{W}_{2}\left(\vartheta_{\text {Blow-up }}, m_{i}\right)\right|>1$,

4. For all $\vartheta$ with $e^{-\ell_{i}} e^{-m_{i}}<\left|\vartheta-\vartheta_{\text {Blow-up }}\right|<\frac{1}{m_{i}^{2}}$, we have $\left|\mathfrak{W}_{2}(\vartheta)\right|>$ $e^{-\ell_{i}} e^{-m_{i}}$

Proof Throughout the proof of Lemma 5.4 we will use the convention that all constants appearing in $\lesssim, \gtrsim, \sim$ and $O$ only depend on $\mathfrak{p}_{0}, l$ and $\delta>0$.

Let $\tilde{m}_{0}>0$. We begin by showing 1 . From Proposition 4.1 and (5.30) we have 


$$
\begin{aligned}
\mathfrak{W}\left[u_{\mathrm{Ai} 2}, u_{\infty}\right]\left(m_{i}, \vartheta\right) & =u_{\mathrm{Ai} 2}\left(r^{*}=l \pi / 2, m_{i}\right) \\
& =\hat{f}_{2}^{\frac{1}{4}}\left(r_{2}^{*}\right) \hat{f}_{2}^{-\frac{1}{4}}(\pi l / 2)\left\{\operatorname{Ai}\left(m_{i}^{\frac{2}{3}} \xi_{2}(l \pi / 2)\right)+\epsilon_{\mathrm{Ai} 2}\left(m_{i}, l \pi / 2\right)\right\} \\
& =\hat{f}_{2}^{\frac{1}{4}}\left(r_{2}^{*}\right) \hat{f}_{2}^{-\frac{1}{4}}(\pi l / 2)\left\{\mathrm{Ai}\left(-\left(\frac{3}{2} m_{i} \xi_{\infty}^{(i)}\right)^{\frac{2}{3}}\right)+O\left(m_{i}^{-\frac{7}{6}}\right)\right\}
\end{aligned}
$$

uniformly on $\gamma_{\delta}\left(\mathfrak{p}_{0}\right)$. Now, for all $m_{i}>\tilde{m}_{0}$ sufficiently large, we use the asymptotics for the Airy functions as shown in Lemma A.1 to conclude that

$$
\begin{aligned}
& \operatorname{Ai}\left(-\left(\frac{3}{2} m_{i} \xi_{\infty}^{(i)}\right)^{\frac{2}{3}}\right)+O\left(m_{i}^{-\frac{7}{6}}\right) \\
& \quad=\frac{1}{\sqrt{\pi}}\left(\frac{3}{2} m_{i} \xi_{\infty}^{(i)}\right)^{-\frac{1}{6}}\left(\cos \left(m_{i} \xi_{\infty}^{(i)}-\frac{\pi}{4}\right)+O\left(m_{i}^{-1}\right)\right) .
\end{aligned}
$$

Thus, in order to conclude that $\mathfrak{W}\left[u_{\mathrm{Ai} 2}, u_{\infty}\right]\left(m_{i}, \vartheta\right)=0$ for some value on $\gamma_{\delta}\left(\mathfrak{p}_{0}\right)$, we have to vary $\mathfrak{p}(\vartheta) \in \gamma_{\delta}\left(\mathfrak{p}_{0}\right)$ such that $m_{i} \xi_{\infty}^{(i)}$ goes through a period of $\pi$. Here, we also use that all terms in $O\left(m_{i}^{-1}\right)$ depend continuously on $\mathfrak{p}$. Thus, it suffices to let $\xi_{\infty}^{(i)}$ go through a period of $\pi m_{i}^{-1}$. From (5.29) we have

$$
\left|\Gamma \xi_{\infty}^{(i)}\right| \sim 1
$$

uniformly on $\gamma_{\delta}\left(\mathfrak{p}_{0}\right)$. Thus, by potentially choosing $m_{i}>\tilde{m}_{0}$ even larger, there exists a parameter $\vartheta_{\text {Blow-up with }}$

$$
\left|\vartheta_{\text {Blow-up }}-\vartheta\left(\mathfrak{p}_{0}\right)\right| \lesssim \frac{1}{m_{i}}
$$

such that

$$
\mathfrak{W}_{2}\left(\vartheta_{\text {Blow-up }}, m_{i}\right)=0 \text { and } \mathfrak{p}\left(\vartheta_{\text {Blow-up }}\right) \in \gamma_{\delta}\left(\mathfrak{p}_{0}\right)
$$

We finally note that from (5.38) and (5.39) we have that Ai $\left(-\left(\frac{3}{2} m_{i} \xi_{\infty}^{(i)}\right)^{\frac{2}{3}}\right)+$ $\epsilon_{\mathrm{Ai} 2}\left(m_{i}, l \pi / 2\right)=O\left(m_{i}^{-\frac{7}{6}}\right)$ for all $\vartheta$ with $\left|\vartheta-\vartheta_{\text {Blow-up }}\right| \leq m_{i}^{-2}$ in view of (5.40).

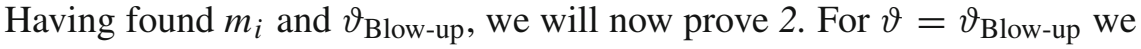
have from Proposition 4.1 and 1 . that 


$$
\begin{aligned}
\left|u_{\mathrm{Ai} 2}^{\prime}(\pi l / 2)\right| & =\hat{f}_{2}^{\frac{1}{4}}\left(r_{2}^{*}\right) \hat{f}_{2}^{-\frac{1}{4}}(\pi l / 2)\left\{\operatorname{Ai}^{\prime}\left(m_{i}^{\frac{2}{3}} \xi_{2}(l \pi / 2)\right) m_{i}^{\frac{2}{3}} \partial_{r^{*}} \xi_{2}(l \pi / 2)+\epsilon_{\mathrm{Ai} 2}^{\prime}\left(m_{i}, l \pi / 2\right)\right\} \\
& =\hat{f}_{2}^{\frac{1}{4}}\left(r_{2}^{*}\right) \hat{f}_{2}^{\frac{1}{4}}(\pi l / 2)\left\{\frac{1}{\sqrt{\pi}} \sin \left(m_{i} \xi_{\infty}^{(i)}-\frac{\pi}{4}\right) m_{i}^{\frac{5}{6}}+O\left(m_{i}^{-\frac{1}{6}}\right)\right\} \\
& \sim \hat{f}_{2}^{\frac{1}{2}}(l \pi / 2) m_{i}^{\frac{5}{6}} .
\end{aligned}
$$

Here, to estimate $\epsilon_{\mathrm{Ai} 2}^{\prime}\left(m_{i}, l \pi / 2\right)$ we used (4.64) and (A.10). We further used Lemma A.1, as well as (4.35) together with $\hat{f}_{2}\left(r_{2}^{*}\right) \sim \hat{f}_{2}(l \pi / 2)$. Finally, we used that $\left|\sin \left(m_{i} \xi_{\infty}^{(i)}-\frac{\pi}{4}\right)\right| \sim 1$ as $\left|\cos \left(m_{i} \xi_{\infty}^{(i)}-\frac{\pi}{4}\right)\right|=O\left(m_{i}^{-1}\right)$. Thus, for $\vartheta=\vartheta_{\text {Blow-up }}$ we have

$$
u_{\mathrm{Ai} 2}=\hat{f}_{2}^{\frac{1}{2}}(l \pi / 2) \alpha_{\infty} u_{\infty} \text { with }\left|\alpha_{\infty}\right| \sim m_{i}^{\frac{5}{6}} .
$$

For 3, we will in fact show the stronger statement that $\left|\Gamma \mathfrak{W}_{2}\left(\vartheta, m_{i}\right)\right|>1$ for all $\vartheta$ with $\left|\vartheta-\vartheta_{\text {Blow-up }}\right| \leq m_{i}^{-2}$. We first recall that

$$
\left|\Gamma \xi_{\infty}^{(i)}\right| \sim 1
$$

on $\gamma_{\delta}\left(\mathfrak{p}_{0}\right)$ in view of (5.29). Now, we take the derivative of (5.38) with respect to $\Gamma$. First we consider the term when $\Gamma$ hits $\hat{f}_{2}^{\frac{1}{4}}\left(r_{2}^{*}\right) \hat{f}_{2}^{-\frac{1}{4}}(\pi l / 2)$. We recall from the proof of $l$ that for $\vartheta$ with $\left|\vartheta-\vartheta_{\text {Blow-up }}\right| \leq m_{i}^{-2}$ we have $\operatorname{Ai}\left(-\left(\frac{3}{2} m_{i} \xi_{\infty}^{(i)}\right)^{\frac{2}{3}}\right)+\epsilon_{\mathrm{Ai} 2}\left(m_{i}, l \pi / 2\right)=O\left(m_{i}^{-\frac{7}{6}}\right)$ such that

$$
\Gamma\left(\hat{f}_{2}^{\frac{1}{4}}\left(r_{2}^{*}\right) \hat{f}_{2}^{-\frac{1}{4}}(\pi l / 2)\right)\left\{\operatorname{Ai}\left(m_{i}^{\frac{2}{3}} \xi_{2}(l \pi / 2)\right)+\epsilon_{\mathrm{Ai} 2}\left(m_{i}, l \pi / 2\right)\right\}=O\left(m_{i}^{-\frac{7}{6}}\right) .
$$

Now, we consider the term when $\Gamma$ hits $\epsilon_{\mathrm{Ai} 2}\left(m_{i}, l \pi / 2\right)$. Using Lemma 5.2 we have that

$$
\hat{f}_{2}^{\frac{1}{4}}\left(r_{2}^{*}\right) \hat{f}_{2}^{-\frac{1}{4}}(\pi l / 2) \Gamma \epsilon_{\mathrm{Ai} 2}\left(m_{i}, l \pi / 2\right)=O\left(m_{i}^{-\frac{1}{6}}\right)
$$

Finally, we consider the term when $\Gamma$ hits $\operatorname{Ai}\left(-\left(\frac{3}{2} m_{i} \xi_{\infty}^{(i)}\right)^{\frac{2}{3}}\right)$. By the chain rule, we directly compute

$$
\left|\Gamma \operatorname{Ai}\left(-\left(\frac{3}{2} m_{i} \xi_{\infty}^{(i)}\right)^{\frac{2}{3}}\right)\right| \sim\left|\operatorname{Ai}^{\prime}\left(-\left(\frac{3}{2} m_{i} \xi_{\infty}^{(i)}\right)^{\frac{2}{3}}\right) m_{i}^{\frac{2}{3}}\right|
$$


in view of (5.45). Similar to the proof of 2., from $\mathrm{Ai}\left(-\left(\frac{3}{2} m_{i} \xi_{\infty}^{(i)}\right)^{\frac{2}{3}}\right)=$ $O\left(m_{i}^{-\frac{7}{6}}\right)$, we have that $\left|\mathrm{Ai}^{\prime}\left(-\left(\frac{3}{2} m_{i} \xi_{\infty}^{(i)}\right)^{\frac{2}{3}}\right)\right| \sim m_{i}^{\frac{1}{4} \frac{2}{3}} \sim m_{i}^{\frac{1}{6}}$. Putting everything together, this shows that

$$
\left|\Gamma \mathfrak{W}_{2}\right| \sim m_{i}^{\frac{1}{6}} m_{i}^{\frac{2}{3}}+O\left(m_{i}^{-\frac{1}{6}}\right)+O\left(m_{i}^{-\frac{7}{6}}\right) \sim m_{i}^{\frac{5}{6}}
$$

for all $\vartheta$ with $\left|\vartheta-\vartheta_{\text {Blow-up }}\right|<m_{i}^{-2}$. In particular, this shows 3. Upon integration this also shows 4 .

Lemma 5.5 There exists a constant $c>0$ (only depending on $\mathfrak{p}_{0}$ and $\delta>0$ ) such that for $\mathfrak{p} \in \gamma_{\delta}\left(\mathfrak{p}_{0}\right)$ we have

$$
\left|\mathfrak{W}\left(u_{\mathrm{Ai} 2}, u_{\mathrm{Bi} 1}\right)\right| \lesssim \hat{f}_{1}^{\frac{1}{2}}\left(r_{1}^{*}\right) e^{-c m_{i}} \quad \text { and } \quad\left|\mathfrak{W}\left(u_{\mathrm{Ai} 2}, u_{\mathrm{Ai} 1}\right)\right| \lesssim \hat{f}_{1}^{\frac{1}{2}}\left(r_{1}^{*}\right) e^{-c m_{i}}
$$

for all $m_{i}$ sufficiently large. Moreover, there exist constants $\alpha_{1}=\alpha_{1}\left(m_{i}\right) \in \mathbb{R}$ and $\beta_{1}=\beta_{1}\left(m_{i}\right) \in \mathbb{R}$ satisfying $\left|\alpha_{1}\right| \lesssim e^{-c m_{i}}$ and $\left|\beta_{1}\right| \lesssim e^{-c m_{i}}$ such that $u_{\mathrm{Ai} 2}=\alpha_{1} u_{\mathrm{Ai} 1}+\beta_{1} u_{\mathrm{Bi} 1}$.

Proof We start by proving $\left|\mathfrak{W}\left(u_{\mathrm{Ai} 2}, u_{\mathrm{Bi} 1}\right)\right| \lesssim \hat{f}_{1}^{\frac{1}{2}}\left(r_{1}^{*}\right) e^{-c m_{i}}$. We evaluate the Wronskian $\mathfrak{W}\left(u_{\mathrm{Ai} 2}, u_{\mathrm{Bi} 1}\right)$ at $r^{*}:=r_{1}^{*}+2 \epsilon\left(\mathfrak{p}_{0}\right)$, where $\epsilon\left(\mathfrak{p}_{0}\right)$ is as in (4.32). By potentially choosing $\delta>0$ smaller, we have that $r_{1}^{*}+2 \epsilon\left(\mathfrak{p}_{0}\right) \geq r_{1}^{*}+\epsilon(\mathfrak{p})$ for all $\mathfrak{p} \in \gamma_{\delta}\left(\mathfrak{p}_{0}\right)$. Then, using standard bounds on Airy functions from Lemma A.1 we obtain

$$
\begin{aligned}
\left|u_{\mathrm{Ai} 2}\left(r_{1}^{*}+2 \epsilon\left(\mathfrak{p}_{0}\right)\right)\right| & \lesssim \frac{1}{m_{i}^{\frac{1}{6}} \xi_{2}^{\frac{1}{4}}\left(r_{1}^{*}+2 \epsilon\left(\mathfrak{p}_{0}\right)\right)} e^{-\frac{2}{3} m_{i} \xi_{2}^{\frac{3}{2}}\left(r_{1}^{*}+2 \epsilon\left(\mathfrak{p}_{0}\right)\right)} \\
\left|u_{\mathrm{Ai} 2}^{\prime}\left(r_{1}^{*}+2 \epsilon\left(\mathfrak{p}_{0}\right)\right)\right| & \lesssim m_{i}^{\frac{1}{6}} \xi_{2}^{\frac{1}{4}}\left(r_{1}^{*}+2 \epsilon\left(\mathfrak{p}_{0}\right)\right) \hat{f}_{2}^{\frac{1}{2}}\left(r_{2}^{*}\right) e^{-\frac{2}{3} m_{i} \xi_{2}^{\frac{3}{2}}\left(r_{1}^{*}+2 \epsilon\left(\mathfrak{p}_{0}\right)\right)} \\
\left|u_{\mathrm{Bi} 1}\left(r_{1}^{*}+2 \epsilon\left(\mathfrak{p}_{0}\right)\right)\right| & \lesssim \frac{1}{m_{i}^{\frac{1}{6}} \xi_{1}^{\frac{1}{4}}\left(r_{1}^{*}+2 \epsilon\left(\mathfrak{p}_{0}\right)\right)} e^{\frac{2}{3} m_{i} \xi_{1}^{\frac{3}{2}}\left(r_{1}^{*}+2 \epsilon\left(\mathfrak{p}_{0}\right)\right)} \\
\left|u_{\mathrm{Bi} 1}^{\prime}\left(r_{1}^{*}+2 \epsilon\left(\mathfrak{p}_{0}\right)\right)\right| & \lesssim m_{i}^{\frac{1}{6}} \xi_{1}^{\frac{1}{4}}\left(r_{1}^{*}+2 \epsilon\left(\mathfrak{p}_{0}\right)\right) \hat{f}_{1}^{\frac{1}{2}}\left(r_{1}^{*}\right) e^{\frac{2}{3} m_{i} \xi_{1}^{\frac{3}{2}}\left(r_{1}^{*}+2 \epsilon\left(\mathfrak{p}_{0}\right)\right)}
\end{aligned}
$$

Now, by choosing $\delta>0$ potentially smaller, in view of (4.36), we have $\frac{\xi_{2}\left(r_{1}^{*}+2 \epsilon\left(\mathfrak{p}_{0}\right)\right)}{\xi_{1}\left(r_{1}^{*}+2 \epsilon\left(\mathfrak{p}_{0}\right)\right)} \geq 2$ for all $\mathfrak{p} \in \gamma_{\delta}\left(\mathfrak{p}_{0}\right)$. Thus, there exists a constant $c=$ 
$c\left(\mathfrak{p}_{0}, \delta\right)>0$ such that

$$
\xi_{2}^{\frac{3}{2}}\left(r_{1}^{*}+2 \epsilon\left(\mathfrak{p}_{0}\right)\right)-\xi_{1}^{\frac{3}{2}}\left(r_{1}^{*}+2 \epsilon\left(\mathfrak{p}_{0}\right)\right) \geq c
$$

for all $\mathfrak{p} \in \gamma_{\delta}\left(\mathfrak{p}_{0}\right)$. Now, the first estimate follows by evaluating the Wronskian $\mathfrak{W}\left(u_{\mathrm{Ai} 2}, u_{\mathrm{Bi} 1}\right)$ at $r^{*}=r_{1}^{*}+2 \epsilon\left(\mathfrak{p}_{0}\right)$ and the fact that $\hat{f}_{1}\left(r_{1}^{*}\right) / \hat{f}_{2}\left(r_{2}^{*}\right) \sim 1$. The second estimate of (5.48) follows in the same manner but it is easier as $u_{\mathrm{Ai} 2}$ is already exponentially small in the region between the turning points $r_{1}^{*}$ and $r_{2}^{*}$ since

$$
\begin{aligned}
\left|u_{\mathrm{Ai} 1}\left(r_{1}^{*}+2 \epsilon\left(\mathfrak{p}_{0}\right)\right)\right| & \lesssim \frac{1}{m_{i}^{\frac{1}{6}} \xi_{1}^{\frac{1}{4}}\left(r_{1}^{*}+2 \epsilon\left(\mathfrak{p}_{0}\right)\right)} e^{-\frac{2}{3} m_{i} \xi_{1}^{\frac{3}{2}}\left(r_{1}^{*}+2 \epsilon\left(\mathfrak{p}_{0}\right)\right)} \\
\left|u_{\mathrm{Ai} 1}^{\prime}\left(r_{1}^{*}+2 \epsilon\left(\mathfrak{p}_{0}\right)\right)\right| & \lesssim m_{i}^{\frac{1}{6}} \xi_{1}^{\frac{1}{4}}\left(r_{1}^{*}+2 \epsilon\left(\mathfrak{p}_{0}\right)\right) \hat{f}_{1}^{\frac{1}{2}}\left(r_{1}^{*}\right) e^{-\frac{2}{3} m_{i} \xi_{1}^{\frac{3}{2}}\left(r_{1}^{*}+2 \epsilon\left(\mathfrak{p}_{0}\right)\right)} .
\end{aligned}
$$

For the second part of the lemma we first note that

$$
\alpha_{1}=\frac{\mathfrak{W}\left(u_{\mathrm{Ai} 2}, u_{\mathrm{Bi} 1}\right)}{\mathfrak{W}\left(u_{\mathrm{Ai} 1}, u_{\mathrm{Bi} 1}\right)}, \beta_{1}=\frac{\mathfrak{W}\left(u_{\mathrm{Ai} 2}, u_{\mathrm{Ai} 1}\right)}{\mathfrak{W}\left(u_{\mathrm{Bi} 1}, u_{\mathrm{Ai} 1}\right)} .
$$

To conclude it suffices to show that

$$
\mathfrak{W}\left(u_{\mathrm{Ai} 1}, u_{\mathrm{Bi} 1}\right) \sim \hat{f}_{1}^{\frac{1}{2}}\left(r_{1}^{*}\right) m_{i}^{\frac{2}{3}} .
$$

In view of the error bounds from (4.59)-(4.62) and the chain rule, we conclude that

$$
\left|\mathfrak{W}_{r^{*}}\left(u_{\mathrm{Ail}}, u_{\mathrm{Bi1}}\right)\right| \sim \hat{f}_{1}^{\frac{1}{2}}\left(r_{1}^{*}\right) m_{i}^{\frac{2}{3}} \mathfrak{W}_{x}(\operatorname{Ai}(x), \operatorname{Bi}(x)) \sim \hat{f}_{1}^{\frac{1}{2}}\left(r_{1}^{*}\right) m_{i}^{\frac{2}{3}}
$$

for all $m_{i}$ sufficiently large.

Now, we are in the position to prove Proposition 5.1.

Proof of Proposition 5.1 Let $m_{0} \in \mathbb{N}$ be arbitrary. Using Lemma 5.4, we let $m_{i}>m_{0}$ and fix $\mathfrak{p}_{\text {Blow-up }} \in \gamma_{\delta}\left(p_{0}\right) \subset \mathcal{U}$ such that $\mathfrak{W}_{2}=0$ and $\left|\Gamma \mathfrak{W}_{2}\right|>1$ as well as $\left|\mathfrak{W}_{2}(\vartheta)\right|>e^{-\ell_{i}} e^{-m_{i}}$ for $e^{-\ell_{i}} e^{-m_{i}}<\left|\vartheta-\vartheta_{\text {Blow-up }}\right|<\frac{1}{m_{i}^{2}}$. We moreover have

$$
u_{\infty}=\alpha_{\infty}^{-1} \hat{f}_{2}^{-\frac{1}{2}}(l \pi / 2) u_{\mathrm{Ai} 2}=\alpha_{\infty}^{-1} \hat{f}_{2}^{-\frac{1}{2}}(l \pi / 2)\left(\alpha_{1} u_{\mathrm{Ai} 1}+\beta_{1} u_{\mathrm{Bi} 1}\right),
$$


where $\left|\alpha_{\infty}\right| \sim m_{i}^{\frac{5}{6}}$. Thus, in view of Lemma 5.5 we have

$$
\begin{aligned}
\left|\mathfrak{W}\left[u_{\infty}, u_{\mathcal{H}^{+}}\right]\right| & =\left|\alpha_{\infty}^{-1} \hat{f}_{2}^{-\frac{1}{2}}(l \pi / 2)\left(\alpha_{1} \mathfrak{W}\left[u_{\mathrm{Ail}}, u_{\mathcal{H}^{+}}\right]+\beta_{1} \mathfrak{W}\left[u_{\mathrm{Bi} 1}, u_{\mathcal{H}^{+}}\right]\right)\right| \\
& \lesssim \hat{f}_{2}^{-\frac{1}{2}}(l \pi / 2) m_{i}^{-\frac{5}{6}} e^{-c m_{i}}\left(\left|\mathfrak{W}\left[u_{\mathrm{Ail}}, u_{\mathcal{H}^{+}}\right]\right|+\left|\mathfrak{W}\left[u_{\mathrm{Bi1}}, u_{\mathcal{H}^{+}}\right]\right|\right)
\end{aligned}
$$

for some constant $c=c\left(\mathfrak{p}_{0}\right)>0$. To estimate $\mathfrak{W}\left[u_{\mathrm{Ail}}, u_{\mathcal{H}^{+}}\right]$and $\mathfrak{W}\left[u_{\mathrm{Bi1}}, u_{\mathcal{H}^{+}}\right]$we infer from Lemma A.1 and (4.55), (4.56) together with the associated error bounds, that

$$
\left|u_{\mathrm{Ai} 1}\right| \lesssim m_{i}^{-\frac{1}{6}}, \quad\left|u_{\mathrm{Ai} 1}^{\prime}\right| \lesssim \hat{f}_{1}^{\frac{1}{2}}\left(r_{1}^{*}\right) m_{i}^{\frac{5}{6}}, \quad\left|u_{\mathrm{Bi} 1}\right| \lesssim m_{i}^{-\frac{1}{6}}, \quad\left|u_{\mathrm{Bi} 1}^{\prime}\right| \lesssim \hat{f}_{1}^{\frac{1}{2}}\left(r_{1}^{*}\right) m_{i}^{\frac{5}{6}}
$$

for all $r^{*}$ sufficiently small and particularly as $r^{*} \rightarrow-\infty$. Moreover, as $r^{*} \rightarrow-\infty$, we have that

$$
u_{\mathcal{H}^{+}}=e^{-i\left(\omega_{-}-\omega_{+}\right) m r^{*}}
$$

such that

$$
\left|\mathfrak{W}\left[u_{\mathrm{Ai} 1}, u_{\mathcal{H}^{+}}\right]\right|,\left|\mathfrak{W}\left[u_{\mathrm{Bi1}}, u_{\mathcal{H}^{+}}\right]\right| \lesssim \hat{f}_{1}^{\frac{1}{2}}\left(r_{1}^{*}\right) m_{i}^{\frac{5}{6}} .
$$

Thus, by potentially choosing $m_{i}$ even larger (i.e. choose $\tilde{m}_{0}$ larger in Lemma 5.4) and noting that $\hat{f}_{2}^{\frac{1}{2}}\left(\frac{\pi}{2} l\right) \sim\left(\omega_{-}-\omega_{+}\right) \sim \hat{f}_{1}^{\frac{1}{2}}\left(r_{1}^{*}\right)$, we have

$$
\left|\mathfrak{W}\left[u_{\infty}, u_{\mathcal{H}^{+}}\right]\right| \lesssim m_{i}^{-\frac{5}{6}} m_{i}^{\frac{5}{6}} e^{-c m_{i}}=e^{-c m_{i}}
$$

and thus,

$$
\left|\mathfrak{W}\left[u_{\infty}, u_{\mathcal{H}^{+}}\right]\right|<e^{-\sqrt{m_{i}}}
$$

for all $m_{i}$ sufficiently large.

Now, we can conclude

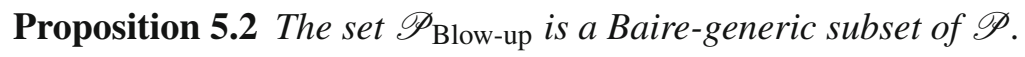

Proof Since $\mathfrak{p}_{0} \in \mathscr{P}$ and $\mathcal{U} \subset \mathscr{P}, \mathcal{U} \ni \mathfrak{p}_{0}$ were arbitrary, Proposition 5.1 shows that for any $m_{0} \in \mathbb{N}$ sufficiently large, the set $U_{m_{0}}$ as defined in Definition 5.2 is dense in $\mathscr{P}$. Since $\mathfrak{W}_{1}, \mathfrak{W}_{2}, \sigma_{1}$ and $\sigma_{2}$ are continuous, $U_{m_{0}}$ is manifestly open. Thus, in view of Baire's theorem [2], $\mathscr{P}_{\text {Blow-up is Baire- }}$ generic and in particular dense. 


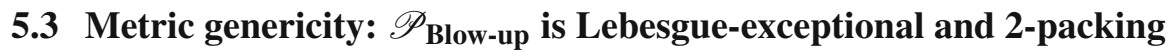 dimensional}

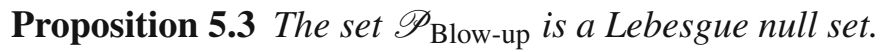

Proof It suffices to show that $\mathscr{P}_{\text {Blow-up }} \cap C$ has vanishing Lebesgue measure (denoted by $|\cdot|$ ) for any closed square $C$ contained in $\mathscr{P}$ with side length less than unity. Let $C$ be such a square contained in $\mathscr{P}$. Throughout the proof, all constants appearing in $\lesssim, \gtrsim, \sim$ and $O$ will only depend on the square $C$. We start by estimating $U(m, \ell) \cap C$ with the co-area formula: We have

$$
|U(m, \ell) \cap C|=\int_{\tilde{\mathfrak{a}} \in\left(\mathfrak{a}_{1}, \mathfrak{a}_{2}\right)} H^{1}(U(m, \ell) \cap C \cap\{\mathfrak{a}=\tilde{\mathfrak{a}}\}) \mathrm{d} \tilde{\mathfrak{a}} .
$$

We recall that $H^{1}$ denotes the one dimensional Hausdorff measure. As $\mid \mathfrak{a}_{2}-$ $\mathfrak{a}_{1} \mid \leq 1$, it suffices to estimate $H^{1}(U(m, \ell) \cap C \cap\{\mathfrak{a}=\tilde{\mathfrak{a}}\})$ uniformly for $\tilde{\mathfrak{a}} \in\left(\mathfrak{a}_{1}, \mathfrak{a}_{2}\right)$.

For each $\tilde{\mathfrak{a}} \in\left(\mathfrak{a}_{1}, \mathfrak{a}_{2}\right)$ we claim that $U(m, \ell) \cap C \cap\{\mathfrak{a}=\tilde{\mathfrak{a}}\}$ can be decomposed into at most $O\left(\mathrm{~m}^{2}\right)$ many subsets, each of which with diameter at most $O\left(e^{-\ell} e^{-m}\right)$. More precisely, for $\vartheta_{1}<\vartheta_{2}$ let $\left(\vartheta_{1}, \tilde{\mathfrak{a}}\right)$ and $\left(\vartheta_{2}, \tilde{\mathfrak{a}}\right)$ be elements of $U(m, \ell) \cap C \cap\{\mathfrak{a}=\tilde{\mathfrak{a}}\}$ in coordinates $(\vartheta, \mathfrak{a})$. Then, we claim that either, $\left|\vartheta_{2}-\vartheta_{1}\right| \leq 2 e^{-\ell} e^{-m}$ or $\left|\vartheta_{2}-\vartheta_{1}\right|>\frac{1}{m^{2}}$.

Indeed, note that $\left(\vartheta_{2}, \tilde{\mathfrak{a}}\right)=\Phi_{\left|\vartheta_{2}-\vartheta_{1}\right|}^{\Gamma}\left(\left(\vartheta_{1}, \tilde{\mathfrak{a}}\right)\right)$. Thus, from the definition of $U(m, \ell)$ and since both, $\left(\vartheta_{1}, \tilde{\mathfrak{a}}\right),\left(\vartheta_{2}, \tilde{\mathfrak{a}}\right) \in U(m, \ell)$, we conclude that

$$
\left|\vartheta_{2}-\vartheta_{1}\right|<2 e^{-\ell} e^{-m} \text { or } \frac{1}{m^{2}}<\left|\vartheta_{2}-\vartheta_{1}\right|
$$

Hence, we decompose $U(m, \ell) \cap C \cap\{\mathfrak{a}=\tilde{\mathfrak{a}}\}$ into $O\left(m^{2}\right)$ many subsets, each of which has diameter at most $O\left(e^{-m} e^{-\ell}\right)$ which is uniform in $\tilde{\mathfrak{a}}$. Thus,

$$
H^{1}(U(m, \ell) \cap C \cap\{\mathfrak{a}=\tilde{\mathfrak{a}}\}) \lesssim m^{2} e^{-\ell} e^{-m}
$$

which implies

$$
|U(m, \ell) \cap C| \lesssim m^{2} e^{-\ell} e^{-m}
$$

Now,

$$
U_{m, C}:=\bigcup_{m \leq \ell \leq m^{2}} U(m, \ell) \cap C
$$


satisfies

$$
\left|U_{m, C}\right| \lesssim e^{-m}
$$

Using the definition of $\mathscr{P}_{\text {Blow-up }}$ from Definition 5.3 we compute

$$
\begin{aligned}
\mathscr{P}_{\text {Blow-up }} \cap C & =\left(\bigcap_{m_{0} \in \mathbb{N}} U_{m_{0}}\right) \cap C=\bigcap_{m_{0} \in \mathbb{N}}\left(U_{m_{0}} \cap C\right) \\
& =\bigcap_{m_{0} \in \mathbb{N}}\left(\left(\bigcup_{m \geq m_{0}} \bigcup_{m \leq \ell \leq m^{2}} U(m, \ell)\right) \cap C\right) \\
& =\bigcap_{m_{0} \in \mathbb{N}}\left(\bigcup_{m \geq m_{0}} \bigcup_{m \leq \ell \leq m^{2}}(U(m, \ell) \cap C)\right) \\
& =\bigcap_{m_{0} \in \mathbb{N}} \bigcup_{m \geq m_{0}} U_{m, C}=\limsup _{m \rightarrow \infty} U_{m, C} .
\end{aligned}
$$

With (5.71) we conclude

$$
\left|\mathscr{P}_{\text {Blow-up }} \cap C\right|=0
$$

in view of the Borel-Cantelli lemma.

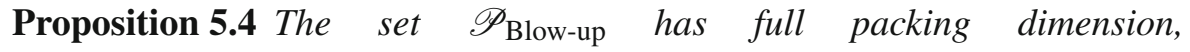
i.e. $\operatorname{dim}_{P}\left(\mathscr{P}_{\text {Blow-up }}\right)=2$.

Proof This follows from Proposition 5.2 and [36, Corollary 3.10].

\section{Construction of the initial data}

Having constructed the set $\mathscr{P}_{\text {Blow-up }}$, we will turn to the problem of showing blow-up. We begin by fixing an arbitrary parameter

$$
\mathfrak{p}=(\mathfrak{m}, \mathfrak{a}) \in \mathscr{P}_{\text {Blow-up }}
$$

which we keep fixed through the rest of the paper, i.e. throughout Sects. 6, 7, 8 and 9. This also fixes the mass $M=\mathfrak{m} l / \sqrt{3}$ and angular momentum $a=$ $\mathfrak{a} l / \sqrt{3}$. As stated in the conventions in Sect. 2.3, all constants appearing in $\lesssim$, $\gtrsim, \sim$ and $O$ will now depend on $\mathfrak{p}$ as fixed in (6.1) (and on $l>0$ as fixed in (2.6)) throughout Sects. 6, 7, 8 and 9.

By construction of $\mathscr{P}_{\text {Blow-up }}$ and since $\mathfrak{p} \in \mathscr{P}_{\text {Blow-up }}$, there exists an infinite sequence

$$
m_{i} \rightarrow \infty, \quad \ell_{i} \rightarrow \infty
$$


with

$$
\left|\mathfrak{W}\left[u_{\mathcal{H}^{+}}, u_{\infty}\right]\left(\omega=\omega_{-} m_{i}, m_{i}, \ell_{i}\right)\right|<e^{-\sqrt{m_{i}}}
$$

and

$$
\frac{\lambda_{m_{i} \ell_{i}}\left(a \omega_{-} m_{i}\right)}{m_{i}^{2}} \in\left(\sigma_{1}(\mathfrak{p}), \sigma_{2}(\mathfrak{p})\right) .
$$

Without loss of generality we also assume that all $m_{i}$ are taken sufficiently large, i.e.

$$
m_{i} \geq m_{0}
$$

for all $i \in \mathbb{N}$ and for a sufficiently large $m_{0}=m_{0}(\mathfrak{p}) \in \mathbb{N}$ only depending on the choice of $\mathfrak{p}$.

We will now carefully choose initial data for (1.2) with compact support in $K$, which we define in the following.

Lemma 6.1 There exists a compact interval $K \subset\left(-\infty, \frac{\pi}{2} l\right)$, an $\epsilon>0$ and a constant $c>0$ such that for every $i \in \mathbb{N}$, there exists a subinterval $K_{i}=$ $\left[r_{i}^{*}-\frac{c}{m_{i}}, r_{i}^{*}+\frac{c}{m_{i}}\right] \subset K$ with

$$
u_{\infty}^{\omega_{-}}:=u_{\infty}\left(\omega=\omega_{-} m_{i}, m_{i}, \ell_{i}, r^{*}\right) \geq \frac{\epsilon}{m_{i}}
$$

for all $r^{*} \in K_{i}$. Moreover, we choose $K$ such that inf $K>3 r_{+}$.

Proof By Definition 4.1, $u_{\infty}^{\omega_{-}}=u_{\infty}\left(\omega=\omega_{-} m_{i}, m_{i}, \ell_{i}, r^{*}\right)$ is a solution to (2.47), i.e. a solution to

$$
-u^{\prime \prime}+\left(m_{i}^{2} V_{\text {main }}+V_{1}\right) u=0,
$$

where

$$
\begin{aligned}
V_{\text {main }} & =\frac{\Delta}{\left(r^{2}+a^{2}\right)^{2}}\left(\frac{\lambda_{m \ell}\left(a \omega_{-} m_{i}\right)}{m_{i}^{2}}+\omega_{-}^{2} a^{2}-2 a \omega_{-} \Xi\right)-\left(\omega_{-}-\omega_{r}\right)^{2}, \\
V_{1} & =\frac{-\Delta^{2} 3 r^{2}}{\left(r^{2}+a^{2}\right)^{4}}-\Delta \frac{5 \frac{r^{4}}{l^{2}}+3 r^{2}\left(1+\frac{a^{2}}{l^{2}}\right)-4 M r+a^{2}}{\left(r^{2}+a^{2}\right)^{3}}-\frac{2 \Delta}{l^{2}} \frac{1}{r^{2}+a^{2}}
\end{aligned}
$$


with $u_{\infty}^{\omega_{-}}(l \pi / 2)=0$ and $u_{\infty}^{\omega_{-}{ }^{\prime}}(l \pi / 2)=1$. Since

$$
\frac{\lambda_{m_{i} \ell_{i}}\left(a \omega_{-} m_{i}\right)}{m_{i}^{2}} \in\left(\sigma_{1}(\mathfrak{p}), \sigma_{2}(\mathfrak{p})\right)
$$

there exists a $\delta>0$ and a $\tilde{r}_{2}^{*}$ such that

$$
V_{\text {main }}<-\delta \text { for } r^{*} \in\left[\tilde{r}_{2}^{*}, l \frac{\pi}{2}\right)
$$

see Lemma 4.2. Without loss of generality we can assume that $\tilde{r}_{2}^{*}>r^{*}(r=$ $3 r_{+}$). In particular, for $m_{i}$ sufficiently large (take $m_{0}(\mathfrak{p})$ possibly larger in (6.5)), we have that

$$
V_{\text {main }}+V_{1} m_{i}^{-2}<-\delta
$$

for $r^{*} \in\left[\tilde{r}_{2}^{*}, \frac{\pi}{2} l\right)$. Now, let $K:=\left[\tilde{r}_{2}^{*}, r_{3}^{*}\right] \subset\left(\tilde{r}_{2}^{*}, \frac{\pi}{2} l\right)$ be a compact subinterval for $r_{3}^{*}>r_{2}^{*}$ fixed, e.g. $r_{3}^{*}=\frac{1}{2}\left(r_{2}^{*}+l \frac{\pi}{2}\right)$. In the region $\left[\tilde{r}_{2}^{*}, \frac{\pi}{2} l\right)$, the smooth potential $V_{\text {main }}$ satisfies

$$
V_{\text {main }}<-\delta,\left|V_{\text {main }}^{\prime}\right| \lesssim 1 \text { and }\left|V_{\text {main }}^{\prime \prime}\right| \lesssim 1
$$

Moreover, $\left|V_{1}\right| \lesssim 1$ uniformly in $\left[\tilde{r}_{2}^{*}, \frac{\pi}{2} l\right)$. This allows us to approximate $u_{\infty}^{\omega_{-}}$ via a WKB approximation. First, we introduce the error-control function

$$
F_{\infty}\left(r^{*}\right):=\int_{r^{*}}^{\frac{\pi}{2} l}\left|V_{\text {main }}\right|^{-\frac{1}{4}} \frac{\mathrm{d}^{2}}{\mathrm{~d} y^{2}}\left(\left|V_{\text {main }}\right|^{-\frac{1}{4}}\right)-\frac{V_{1}}{\left|V_{\text {main }}\right|^{\frac{1}{2}}} \mathrm{~d} y
$$

and note that $F_{\infty}\left(\frac{\pi}{2} l\right)=0$. In view of the above bounds on $V_{\text {main }}$ and $V_{1}$ we obtain

$$
\mathcal{V}_{\tilde{r}_{2}^{*}, \frac{\pi}{2} l}\left(F_{\infty}\right) \lesssim 1
$$

Hence, using the boundary conditions $u_{\infty}^{\omega_{-}}\left(\frac{\pi}{2} l\right)=0, \frac{\mathrm{d}}{\mathrm{d} r^{*}} u_{\infty}^{\omega_{-}}\left(\frac{\pi}{2} l\right)=1$ we obtain from [94, Chapter 6, Theorem 2.2] that the solution $u_{\infty}^{\omega_{-}}$is given as

$$
u_{\infty}^{\omega_{-}}=\frac{A}{m_{i}\left|V_{\text {main }}\right|^{\frac{1}{4}}} \sin \left(-m_{i} \int_{r^{*}}^{\frac{\pi}{2} l} \sqrt{\left|V_{\text {main }}\right|} \mathrm{d} y\right)\left(1+\epsilon_{u_{\infty}}\right)
$$


where $A=\left|V_{\text {main }}^{-\frac{1}{4}}\left(r^{*}=l \frac{\pi}{2}\right)\right|$ satisfies $|A| \sim 1$ and $\epsilon_{u_{\infty}}$ satisfies $\epsilon_{u_{\infty}}\left(\frac{\pi}{2} l\right)=$ $\epsilon_{u_{\infty}}^{\prime}\left(\frac{\pi}{2} l\right)=0$ as well as

$$
\left|\epsilon_{u_{\infty}}\right|, \frac{\left|\epsilon_{u_{\infty}}^{\prime}\right|}{2 m_{i}\left|V_{\text {main }}\right|^{\frac{1}{2}}} \lesssim \frac{\left.\mathcal{V}_{\tilde{r}_{2}^{*}, \frac{\pi}{2} l} l F_{\infty}\right)}{m_{i}} \lesssim \frac{1}{m_{i}}
$$

Indeed, note that the condition $u_{\infty}^{\omega_{-}}\left(\frac{\pi}{2} l\right)=0, \frac{\mathrm{d}}{\mathrm{d} r^{*}} u_{\infty}^{\omega_{-}}\left(\frac{\pi}{2} l\right)=1$ and $\epsilon_{u_{\infty}}\left(\frac{\pi}{2} l\right)=$ $\epsilon_{u_{\infty}}^{\prime}\left(\frac{\pi}{2} l\right)=0$ force $u_{\infty}^{\omega_{-}}$to be of the form (6.16). Now, since $u_{\infty}^{\omega_{-}}$oscillates with period proportional to $m_{i}$ in view of (6.16), there exists a compact subinterval $K_{i} \subset K$ of the form $K_{i}=\left[r_{i}^{*}-\frac{c}{m_{i}}, r_{i}^{*}+\frac{c}{m_{i}}\right]$ for some $c>0$ such that for all $r^{*} \in K_{i}$, we have

$$
u_{\infty}^{\omega_{-}}\left(r^{*}, m_{i}, \ell_{i},\right) \geq \frac{\epsilon}{m_{i}} .
$$

We are now in the position to define our initial data which will be supported in the compact set $K$ as defined in Lemma 6.1. We assume without loss of generality that all $m_{i}$ are sufficiently large such that we can apply Lemma 6.1. First, let $\chi: \mathbb{R} \rightarrow[0,1]$ be a smooth bump function satisfying $\chi=0$ for $|x| \geq 1$ and $\chi=1$ for $|x| \leq \frac{1}{2}$. Then, for $i \in \mathbb{N}$ we set

$$
\chi_{i}:(-\infty, l \pi / 2) \rightarrow[0,1], r^{*} \mapsto \chi\left(c^{-1} m_{i}\left(r^{*}-r_{i}^{*}\right)\right) .
$$

Definition 6.1 Let $m_{i}, \ell_{i}$ be as in (6.2). For each $i \in \mathbb{N}$, let $K_{i} \subset K$ be the associated subinterval as specified in Lemma 6.1 and let $\chi_{i}$ defined as in (6.19). Then, we define initial data on $\Sigma_{0}$ as

$$
\begin{aligned}
& \psi \uparrow_{\Sigma_{0}}=\Psi_{0}:=0, \\
& n_{\Sigma_{0}} \psi \uparrow_{\Sigma_{0}}(r, \theta, \phi)=\Psi_{1}(r, \theta, \phi):=\sum_{i \geq i_{0}} e^{-m_{i}^{\frac{1}{3}}} \psi_{i}(r, \theta, \phi),
\end{aligned}
$$

where

$$
\psi_{i}(r, \theta, \phi)=\frac{\sqrt{r^{2}+a^{2}} \chi_{i}\left(r^{*}(r)\right)}{-2 \Sigma \sqrt{-g^{t t}}(r, \theta) u_{\infty}^{\omega_{-}}\left(r^{*}(r)\right)} S_{m_{i} \ell_{i}}\left(a \omega_{-} m_{i}, \cos \theta\right) e^{i m_{i} \phi} .
$$

Having set up the initial data we proceed to 
Definition 6.2 Throughout the rest of Sects. 7, 8 and 9 we define $\psi \in$ $C^{\infty}\left(\mathcal{M}_{\text {Kerr-AdS }} \backslash \mathcal{C H}\right)$ to be the unique smooth solution to (1.2) of the mixed Cauchy-boundary value problem with vanishing data on $\mathcal{H}_{L} \cup \mathcal{B}_{\mathcal{H}}$, Dirichlet boundary conditions at infinity and the initial data $\left(\Psi_{0}, \Psi_{1}\right) \in C_{c}^{\infty}\left(\Sigma_{0}\right)$ posed on $\Sigma_{0}$ specified in Definition 6.1. This is well-posed in view of Theorem 2.

Remark 6.1 By a domain of dependence argument one can also view $\psi$ as arising from smooth and compactly supported initial data posed on a spacelike hypersurface connecting both components of $\mathcal{I}$ as depicted in Fig. 2.

Remark 6.2 We note that our initial data are only supported on the positive azimuthal frequencies $m=m_{i}$. The same will apply to the arising solution $\psi$.

In the following we define the quantity $a_{\mathcal{H}}$ from our initial data. This $a_{\mathcal{H}}$ will turn out (at least in a limiting sense) to be the (generalized) Fourier transform of the solution $\psi \Gamma_{\mathcal{H}}$ along the event horizon.

Definition 6.3 For the initial data $\Psi_{0}, \Psi_{1}$ as in Definition 6.1 we define

$$
\begin{aligned}
a_{\mathcal{H}}(\omega, m, \ell):= & \frac{1}{\sqrt{2 \pi} \mathfrak{W}\left[u_{\mathcal{H}^{+}}, u_{\infty}\right]} \int_{r_{+}}^{\infty} \int_{\mathbb{S}^{2}}\left\{\frac{\Sigma}{\sqrt{r^{2}+a^{2}}} u_{\infty} e^{-i m \phi} S_{m \ell}(a \omega, \cos \theta)\right. \\
& \left.\times\left(-2 \sqrt{-g^{t t}} \Psi_{1}-i \omega g^{t t} \Psi_{0}\right)\right\} \mathrm{d} \sigma_{\mathbb{S}^{2}} \mathrm{~d} r .
\end{aligned}
$$

Now, we will show that $a_{\mathcal{H}}$ has "peaks" at the interior scattering poles $\omega=$ $\omega_{-} m$ for infinitely many $m$. This is a consequence of our careful choice of initial data. We formulate this in

Lemma 6.2 For $a_{\mathcal{H}}$ as in Definition 6.1 we have

$$
a_{\mathcal{H}}\left(\omega=\omega_{-} m, m, \ell\right)=a_{\mathcal{H}}\left(\omega=\omega_{-} m_{i}, m_{i}, \ell_{i}\right) \delta_{m m_{i}} \delta_{\ell \ell_{i}},
$$

where

$$
\left|a_{\mathcal{H}}\left(\omega=\omega_{-} m_{i}, m_{i}, \ell_{i}\right)\right| \gtrsim e^{\frac{1}{2} \sqrt{m_{i}}}
$$

for $\left(m_{i}, \ell_{i}\right)$ as in (6.2).

Proof As $\Psi_{0}=0$, we compute

$$
\begin{aligned}
& \int_{r_{+}}^{\infty} \int_{\mathbb{S}^{2}} \frac{\Sigma}{\sqrt{r^{2}+a^{2}}} u_{\infty}^{\omega_{-}} e^{-i m \phi} S_{m \ell}(a \omega-m, \cos \theta)\left(-2 \sqrt{-g^{t t}}\right) \Psi_{1} \mathrm{~d} \sigma_{\mathbb{S}^{2}} \mathrm{~d} r \\
& =e^{-m_{i}^{\frac{1}{3}}} \delta_{m m_{i}} \delta_{\ell \ell_{i}} \int_{r_{+}}^{\infty} \chi_{i}(r) \mathrm{d} r \sim e^{-m_{i}^{\frac{1}{3}}} m_{i}^{-1} \delta_{m m_{i}} \delta_{\ell \ell_{i}} .
\end{aligned}
$$


To conclude we use that from (6.3) we have

$$
\left|\mathfrak{W}\left[u_{\mathcal{H}^{+}}, u_{\infty}\right]\left(\omega=\omega_{-} m_{i}, m_{i}, \ell_{i}\right)\right|<e^{-\sqrt{m}_{i}} .
$$

\section{Exterior analysis: from the initial data to the event horizon}

\subsection{Cut-off in time and inhomogeneous equation}

We will now consider the $\psi$ as defined in Sect. 6. The goal of this section is to determine the Fourier transform of $\psi$ along the event horizon. To do so we will first take a time cut-off of $\psi$. To do so, we let

$$
\chi: \mathbb{R} \rightarrow[0,1]
$$

be a smooth and monotone cut-off function with $\chi(x)=0$ for $x \leq 0, \chi(x)=1$ for $x \geq 1$. Now, define $\chi_{\epsilon}^{R}(v):=\chi(v / \epsilon) \chi(R-v)$ such that $\chi_{\epsilon}^{R} \rightarrow \nVdash_{(0, \infty)}$ pointwise as $\epsilon \rightarrow 0$ and $R \rightarrow \infty$. Moreover,

$$
\partial_{v}(\chi(v / \epsilon)) \rightarrow \delta_{0}(v) \quad \text { and } \quad \partial_{v}^{2}(\chi(v / \epsilon)) \rightarrow \delta_{0}^{\prime}(v)
$$

as $\epsilon \rightarrow 0$ in the sense of distributions. On $\mathcal{R} \cup \mathcal{H}_{R}$ we set

$$
\psi_{\epsilon}^{R}\left(v, r, \theta, \tilde{\phi}_{+}\right):=\psi\left(v, r, \theta, \tilde{\phi}_{+}\right) \chi_{\epsilon}^{R}(v) \text { and } \psi^{R}:=\psi\left(v, r, \theta, \tilde{\phi}_{+}\right) \chi(R-v)
$$

and note that $\psi_{\epsilon}^{R}$ is smooth and compactly supported in $v$ and satisfies the inhomogeneous equation

$$
\square_{g_{\text {Kerr-AdS }}} \psi_{\epsilon}^{R}+\frac{2}{l^{2}} \psi_{\epsilon}^{R}=F_{\epsilon}^{R}:=2\left(\partial_{v} \chi_{\epsilon}^{R}\right)(\nabla v) \psi+\psi \square_{g_{\text {Kerr-AdS }}} \chi_{\epsilon}^{R} .
$$

Analogously, $\psi^{R}$ satisfies the inhomogeneous equation with

$$
\square_{g_{\text {Kerr-AdS }}} \psi^{R}+\frac{2}{l^{2}} \psi^{R}=F^{R}:=2\left(\partial_{v} \chi^{R}\right)(\nabla v) \psi+\psi \square_{g_{\text {Kerr-AdS }}} \chi^{R} .
$$

As in [63, Sect. 5.1] we have

$$
\begin{aligned}
& \left|F^{R}\right|^{2} r^{2} \lesssim \frac{1}{r^{2}}\left|\partial_{v} \psi\right|^{2}+r^{2}\left|\partial_{r} \psi\right|^{2}+\left.|\not| \psi\right|^{2}+|\psi|^{2}, \\
& \left|F_{\epsilon}^{R}\right|^{2} r^{2} \lesssim \frac{1}{\epsilon^{2} r^{2}}\left|\partial_{v} \psi\right|^{2}+r^{2}\left|\partial_{r} \psi\right|^{2}+|\not \nabla \psi|^{2}+\frac{1}{\epsilon^{2}}|\psi|^{2} .
\end{aligned}
$$


In view of our coordinates, we also have that for each $r>r_{+}$, the function $\psi_{\epsilon}^{R}(t, r, \theta, \phi)$ is compactly supported in $\mathbb{R}_{t}$ with values in $C^{\infty}\left(\mathbb{S}^{2}\right)$. This allows us to apply Carter's separation of variables to express $\psi_{\epsilon}^{R}$ as

$$
\psi_{\epsilon}^{R}(t, r, \theta, \phi)=\frac{1}{\sqrt{2 \pi}} \int_{\mathbb{R}} \mathfrak{F}\left[\psi_{\epsilon}^{R}\right](\omega, r, \theta, \phi) e^{-i \omega t} \mathrm{~d} \omega,
$$

where for each $r>r_{+}$,

$$
\mathfrak{F}\left[\psi_{\epsilon}^{R}\right](\omega, r, \theta, \phi):=\frac{1}{\sqrt{2 \pi}} \int_{\mathbb{R}} \psi_{\epsilon}^{R}(t, r, \theta, \phi) e^{i \omega t} \mathrm{~d} t
$$

is a Schwartz function on $\mathbb{R}_{\omega}$ with values in $C^{\infty}\left(\mathbb{S}^{2}\right)$. For the definition of Fréchet space-valued Schwartz functions refer to [111, p. 533] or to [109, Definition 3] by L. Schwartz himself. Note however that we will only use Fréchet space-valued Schwartz functions in a qualitative way and in view of this we will not go into more details. We further decompose $\mathfrak{F}\left[\psi_{\epsilon}^{R}\right](\omega, r, \theta, \phi)$ in (generalized) spheroidal harmonics

$$
\mathfrak{F}\left[\psi_{\epsilon}^{R}\right](\omega, r, \theta, \phi)=\sum_{m \ell} \hat{\psi_{\epsilon}^{R}}(\omega, m, \ell, r) S_{m \ell}(a \omega, \cos \theta) e^{i m \phi},
$$

where

$$
\hat{\psi_{\epsilon}^{R}}(\omega, m, \ell, r):=\int_{\mathbb{S}^{2}} \mathfrak{F}\left[\psi_{\epsilon}^{R}\right](\omega, r, \theta, \phi) e^{-i m \phi} S_{m \ell}(a \omega, \cos \theta) \mathrm{d} \sigma_{\mathbb{S}^{2}}
$$

is smooth in $\omega$ and $r>r_{+}$for fixed $m$ and $\ell$ and moreover

$$
\hat{\psi_{\epsilon}^{R}} \in L^{2}\left(\mathbb{R}_{\omega} \times \mathbb{Z}_{m} \times \mathbb{Z}_{\ell \geq|m|} ; C^{\infty}(\tilde{r}, \infty)\right)
$$

in view of Plancherel's theorem for every $\tilde{r}>r_{+}$. Equivalently, we have

$$
\begin{aligned}
& \hat{\psi_{\epsilon}^{R}}(\omega, m, \ell, r) \\
& \quad=\frac{1}{\sqrt{2 \pi}} \int_{\mathbb{R}} \int_{\mathbb{S}^{2}} \psi_{\epsilon}^{R}(t, r, \theta, \phi) e^{i \omega t} e^{-i m \phi} S_{m \ell}(a \omega, \cos \theta) \mathrm{d} \sigma_{\mathbb{S}^{2}} \mathrm{~d} t
\end{aligned}
$$

for each $r>r_{+}$. Now, note that $\psi_{\epsilon}^{R}\left(v, r, \theta, \tilde{\phi}_{+}\right)$is smooth and compactly supported on $\mathbb{R}_{v}$ all the way to $r=r_{+}$and thus, takes values in the space $C^{\infty}\left(\left[r_{+}, \infty\right)_{r} \times \mathbb{S}_{\theta, \tilde{\phi}_{+}}^{2}\right)$. After a change of coordinates in (7.13) we obtain that

$$
\hat{\psi}_{\epsilon}^{R}(\omega, r, m, \ell) e^{i\left(\omega-\omega_{+} m\right) r^{*}}
$$


extends smoothly to $r=r_{+}\left(r^{*} \rightarrow-\infty\right)$. Similarly to the above, we have

$$
\begin{aligned}
& \widehat{\Sigma F_{\epsilon}^{R}}(\omega, m, \ell, r) \\
& =\frac{1}{\sqrt{2 \pi}} \int_{\mathbb{R}} \int_{\mathbb{S}^{2}} \Sigma F_{\epsilon}^{R}(t, r, \theta, \phi) e^{i \omega t} e^{-i m \phi} S_{m \ell}(a \omega, \cos \theta) \mathrm{d} \sigma_{\mathbb{S}^{2}} \mathrm{~d} t .
\end{aligned}
$$

Now, we define

$$
u_{\epsilon}^{R}=u_{\epsilon}^{R}(\omega, m, \ell, r):=\left(r^{2}+a^{2}\right)^{\frac{1}{2}} \hat{\psi}_{\epsilon}^{R}(\omega, m, \ell, r)
$$

and

$$
H_{\epsilon}^{R}(\omega, m, \ell, r):=\frac{\Delta}{\left(r^{2}+a^{2}\right)^{\frac{3}{2}}} \widehat{\Sigma F_{\epsilon}^{R}}(\omega, m, \ell, r) .
$$

Since $\hat{\psi_{\epsilon}^{R}}$ defined in (7.11) is smooth, we have that $u_{\epsilon}^{R}$ as defined in (7.16) is a smooth function of $\omega$ and $r>r_{+}$. Moreover, we can also differentiate under the integral sign in (7.13) and since $\psi_{\epsilon}^{R}$ satisfies (7.4), we have that $u_{\epsilon}^{R}$ satisfies the inhomogeneous radial o.d.e.

$$
-u_{\epsilon}^{R^{\prime \prime}}+\left(V-\omega^{2}\right) u_{\epsilon}^{R}=H_{\epsilon}^{R}
$$

pointwise for each $\omega, m, \ell$ on $r^{*} \in\left(-\infty, \frac{\pi}{2} l\right]$, where we recall that ${ }^{\prime}=\frac{\mathrm{d}}{\mathrm{d} r^{*}}$.

\subsection{Estimates for the inhomogeneous radial o.d.e.}

Lemma 7.1 The solution $u_{\epsilon}^{R}$ as defined in (7.16) satisfies the boundary conditions

$$
\begin{aligned}
& u_{\epsilon}^{R}=0 \text { for } r^{*}=\frac{\pi}{2} l, \\
& u_{\epsilon}^{R^{\prime}}+i\left(\omega-\omega_{+} m\right) u_{\epsilon}^{R}=O(\Delta) \text { as } r^{*} \rightarrow-\infty
\end{aligned}
$$

and the inhomogeneity $H_{\epsilon}^{R}$ defined in (7.18) also satisfies

$$
\begin{aligned}
& H_{\epsilon}^{R}=0 \text { for } r^{*}=\frac{\pi}{2} l \\
& H_{\epsilon}^{R^{\prime}}+i\left(\omega-\omega_{+} m\right) H_{\epsilon}^{R}=O(\Delta) \text { as } r^{*} \rightarrow-\infty .
\end{aligned}
$$


Proof To see (7.19) note that

$$
\begin{aligned}
\left|u_{\epsilon}^{R}\right| & \leq\left(r^{2}+a^{2}\right)^{\frac{1}{2}}\left|\hat{\psi}_{\epsilon}^{R}\right| \\
& =\left|\int_{\mathbb{R}} \int_{\mathbb{S}^{2}}\left(r^{2}+a^{2}\right)^{\frac{1}{2}} \psi_{\epsilon}^{R}(r, t, \theta, \phi) e^{i \omega t} S_{m \ell}(a \omega, \cos \theta) e^{-i m \phi} \mathrm{d} \sigma \mathrm{d} t\right| .
\end{aligned}
$$

In view of the compact support of $\psi_{\epsilon}^{R}$ in $t$, it suffices to show that the pointwise limit

$$
\lim _{r \rightarrow \infty} r \psi_{\epsilon}^{R}(t, r, \theta, \phi)=0
$$

holds true. But this follows from the fact that $\psi_{\epsilon}^{R} \in C H_{\text {AdS }}^{1}$-a consequence of the well-posedness in Theorem 2.

For (7.20), we use (7.14) to see that $\partial_{r}\left(\hat{\psi}_{\epsilon}^{R}(\omega, r, m, \ell) e^{i\left(\omega-\omega_{+} m\right) r^{*}}\right)$ extends smoothly to $r=r_{+}$. Thus, using $\partial_{r}=\frac{r^{2}+a^{2}}{\Delta} \partial_{r^{*}}$, we infer that

$$
u_{\epsilon}^{R^{\prime}}+i\left(\omega-\omega_{+} m\right) u_{\epsilon}^{R}=O(\Delta) \text { as } r^{*} \rightarrow-\infty .
$$

Analogously, we obtain (7.21) and (7.22).

Lemma 7.2 We represent $u_{\epsilon}^{R}$ as

$$
u_{\epsilon}^{R}\left(r^{*}\right)=\frac{1}{\mathfrak{W}\left[u_{\mathcal{H}^{+}}, u_{\infty}\right]}\left\{u_{\mathcal{H}^{+}} \int_{r^{*}}^{\frac{\pi}{2}} u_{\infty} H_{\epsilon}^{R} \mathrm{~d} y+u_{\infty} \int_{-\infty}^{r^{*}} u_{\mathcal{H}^{+}} H_{\epsilon}^{R} \mathrm{~d} y\right\}
$$

Moreover,

$$
\lim _{r_{*} \rightarrow-\infty} u_{\epsilon}^{R}\left(r^{*}\right) e^{i\left(\omega-\omega_{+} m\right) r^{*}}=a_{\epsilon, \mathcal{H}}^{R},
$$

where $a_{\epsilon, \mathcal{H}}^{R}$ is defined as

$$
a_{\epsilon, \mathcal{H}}^{R}:=\frac{1}{\mathfrak{W}\left[u_{\mathcal{H}^{+}}, u_{\infty}\right]} \int_{-\infty}^{\frac{\pi}{2}} u_{\infty} H_{\epsilon}^{R} \mathrm{~d} y
$$

Proof First, since there do not exist pure mode solutions as shown in [63, Theorem 1.3], the Wronskian $\mathfrak{W}\left[u_{\mathcal{H}^{+}}, u_{\infty}\right]$ never vanishes. Thus, (7.26) is well-defined and in view of the boundary conditions of $u_{\epsilon}^{R}$ and $H_{\epsilon}^{R}$ as shown 
in Lemma 7.1, a direct computation shows (7.26). To show (7.27) we first note that that

$$
\left|u_{\infty}\right| \leq C_{m \ell \omega}\left|r^{*}\right|
$$

for all $r^{*}$. Indeed, (7.29) holds true as for each $\omega, m, \ell$ there exist constants $a_{s}, a_{c}$ only depending on the $\omega, m, \ell$ such that

$$
u_{\infty}=a_{s} u_{s}+a_{c} u_{c}
$$

where $u_{s}$ and $u_{c}$ are solutions to the radial o.d.e. satisfying $u_{s} \sim \frac{\sin \left(\left(\omega-\omega_{+} m\right) r^{*}\right)}{\omega-\omega_{+} m}$ and $u_{c} \sim \cos \left(\left(\omega-\omega_{+} m\right) r^{*}\right)$ as $r^{*} \rightarrow-\infty$. For $\omega \neq \omega_{+} m, u_{s}$ and $u_{c}$ are defined as $u_{s}=\frac{1}{2 i} \frac{u_{\mathcal{H}^{-}}-u_{\mathcal{H}^{+}}}{\omega-\omega_{+} m}$ and $u_{c}=\frac{1}{2 i}\left(u_{\mathcal{H}^{-}}+u_{\mathcal{H}^{+}}\right)$, where $u_{\mathcal{H}^{+}}$and $u_{\mathcal{H}^{-}}$ are defined in Definition 4.2. This definition uniquely extends to $\omega=\omega_{+} m$ with the asymptotics $u_{s} \sim r^{*}$ and $u_{c} \sim 1$ as $r^{*} \rightarrow-\infty$. In particular, $\mathfrak{W}\left(u_{s}, u_{c}\right)=-1$ for all $\omega, m, \ell$ which justifies (7.30).

We now obtain (7.27) since

$$
\begin{aligned}
& \limsup _{r^{*} \rightarrow-\infty}\left|u_{\infty} \int_{-\infty}^{r^{*}} u_{\mathcal{H}^{+}} H_{\epsilon}^{R} \mathrm{~d} y\right|^{2} \\
& \leq C_{m \ell \omega}^{2} \limsup _{r^{*} \rightarrow-\infty}\left(\left|r^{*}\right| \int_{r_{+}}^{r\left(r^{*}\right)} \frac{\left|\widehat{\Sigma F_{\epsilon}^{R}}\right|^{2}}{r^{2}+a^{2}} \mathrm{~d} r \int_{-\infty}^{r_{*}}\left|u_{\mathcal{H}^{+}}\right|^{2} \frac{\Delta}{r^{2}+a^{2}} \mathrm{~d} y\right) \\
& \leq C_{m \ell \omega}^{2} \limsup _{r^{*} \rightarrow-\infty}\left(\int_{r_{+}}^{r\left(r^{*}\right)} \frac{\left|\widehat{\Sigma F}_{\epsilon}^{R}\right|^{2}}{r^{2}+a^{2}} \mathrm{~d} r \int_{-\infty}^{r_{*}}\left|u_{\mathcal{H}^{+}}\right|^{2} \frac{|y|^{2} \Delta}{r^{2}+a^{2}} \mathrm{~d} y\right)=0
\end{aligned}
$$

because

$$
\int_{r_{+}}^{r\left(r^{*}\right)} \frac{\left|\widehat{\Sigma F_{\epsilon}^{R}}\right|^{2}}{r^{2}+a^{2}} \mathrm{~d} r<\infty, \sup _{r^{*} \in\left(-\infty, r_{1}\right)}\left|u_{\mathcal{H}^{+}}\right|<\infty
$$

and $\left|r^{*}\right|^{2} \Delta$ decays exponentially as $r^{*} \rightarrow-\infty$. In (7.31) we also used that $\mathrm{d} r=\frac{\Delta}{r^{2}+a^{2}} \mathrm{~d} r^{*}$.

Lemma 7.3 The inhomogeneous term $H_{\epsilon}^{R}$ has the pointwise limit

$$
\begin{aligned}
H^{R} & :=\lim _{\epsilon \rightarrow 0} H_{\epsilon}^{R} \\
& =\frac{\Delta}{\left(r^{2}+a^{2}\right)^{\frac{3}{2}}} \frac{1}{\sqrt{2 \pi}} \int_{\mathbb{S}^{2}} \Sigma e^{-i m \phi} S_{m \ell}(a \omega)\left(-2 \sqrt{-g^{t t}} \Psi_{1}-i \omega g^{t t} \Psi_{0}\right) \mathrm{d} \sigma_{\mathbb{S}^{2}}
\end{aligned}
$$




$$
\begin{aligned}
& +\frac{\Delta}{\left(r^{2}+a^{2}\right)^{\frac{3}{2}}} \frac{e^{-i(\omega-\omega+m) r^{*}}}{\sqrt{2 \pi}} \\
& \times \int_{R-1}^{R} \int_{\mathbb{S}^{2}} \Sigma F^{R}\left(v, r, \theta, \tilde{\phi}_{+}\right) e^{i \omega v} e^{-i m \tilde{\phi}_{+}} S_{m \ell}(a \omega) \mathrm{d} \sigma_{\mathbb{S}^{2}} \mathrm{~d} v .
\end{aligned}
$$

In addition,

$$
a_{\epsilon, \mathcal{H}}^{R} \rightarrow a_{\mathcal{H}}^{R}:=\frac{1}{\mathfrak{W}\left[u_{\mathcal{H}^{+}}, u_{\infty}\right]} \int_{-\infty}^{\frac{\pi}{2}} u_{\infty} H^{R} \mathrm{~d} r^{*}
$$

pointwise as $\epsilon \rightarrow 0$.

Moreover, we have

$$
\begin{aligned}
H^{R} \rightarrow H:= & \frac{\Delta}{\left(r^{2}+a^{2}\right)^{\frac{3}{2}}} \frac{1}{\sqrt{2 \pi}} \int_{\mathbb{S}^{2}} \Sigma e^{-i m \phi} S_{m \ell}(a \omega) \\
& \times\left(-2 \sqrt{-g^{t t}} \Psi_{1}-i \omega g^{t t} \Psi_{0}\right) \mathrm{d} \sigma_{\mathbb{S}^{2}}
\end{aligned}
$$

and

$$
a_{\mathcal{H}}^{R} \rightarrow a_{\mathcal{H}}
$$

pointwise as $R \rightarrow \infty$. Recall that $a_{\mathcal{H}}$ is defined in Definition 6.3.

Proof We start with the decomposition $F_{\epsilon}^{R}=F_{\epsilon}+F_{R}$, where the support of $F_{\epsilon}$ is in $\{0 \leq t \leq \epsilon\} \cap\left\{r \geq 2 r_{+}\right\}$for $\epsilon>0$ small enough and the support of $F_{R}$ is in the set $\{R-1 \leq v \leq R\}$.

We first consider $F_{\epsilon}$ and write its (generalized) Fourier transform as

$$
\widehat{\Sigma F_{\epsilon}}(\omega, m, \ell, r)=\frac{1}{\sqrt{2 \pi}} \int_{\mathbb{S}^{2}} \int_{\mathbb{R}} F_{\epsilon}(t, r, \theta, \phi) e^{i \omega t} \mathrm{~d} t \Sigma e^{-i m \phi} S_{m \ell}(a \omega, \cos \theta) \mathrm{d} \sigma_{\mathbb{S}^{2}} .
$$

Recall that for all $\epsilon>0$ sufficiently small, we have that $v(t, r, \theta, \phi)=t$ on the support of $F_{\epsilon}$. Thus,

$$
\begin{aligned}
F_{\epsilon} & =2 \partial_{t}(\chi(t / \epsilon))(\nabla t) \psi+\psi \square_{g_{\text {Kerr-AdS }}} \chi(t / \epsilon) \\
& =-2 \partial_{t}(\chi(t / \epsilon)) \sqrt{-g^{t t}} n_{\Sigma_{t}} \psi+\psi g^{t t} \partial_{t}^{2}(\chi(t / \epsilon)),
\end{aligned}
$$

where $\chi$ is as in (7.1). We also used that for any $f(t, r, \theta, \phi)=f(t)$ only depending on $t$ in Boyer-Lindquist coordinates, we have $\square_{g_{\text {Kerr-AdS }}} f=$ $g^{t t} \partial_{t}^{2} f$. Now, in view of (7.2) we obtain

$$
F_{\epsilon} \rightarrow F_{0}:=-2 \sqrt{-g^{t t}} \delta_{t=0} \Psi_{1}+g^{t t} \delta_{t=0}^{\prime} \Psi_{0}
$$


as $\epsilon \rightarrow 0$ in the sense of distributions (compactly supported distributions) on $\mathbb{R}_{t}$ with values in $C^{\infty}\left(\left(r_{+}, \infty\right) \times \mathbb{S}^{2}\right)$. Hence,

$$
\begin{aligned}
\widehat{\Sigma F_{\epsilon}}(\omega, m, \ell, r) & =\frac{1}{\sqrt{2 \pi}} \int_{\mathbb{S}^{2}} \int_{\mathbb{R}} e^{-i m \phi} S_{m \ell}(a \omega, \cos \theta) F_{\epsilon} e^{i \omega t} \mathrm{~d} t \mathrm{~d} \sigma_{\mathbb{S}^{2}} \\
& \rightarrow \frac{1}{\sqrt{2 \pi}} \int_{\mathbb{S}^{2}} e^{-i m \phi} S_{m \ell}(a \omega, \cos \theta)\left(-2 \sqrt{-g^{t t}} \Psi_{1}-i \omega g{ }^{t t} \Psi_{0}\right) \mathrm{d} \sigma_{\mathbb{S}^{2}}
\end{aligned}
$$

pointwise. (Note that the above pointwise limit can also be shown via integration by parts in $t$ without appealing to distribution theory, cf. (7.43) below.) Thus,

$$
\begin{aligned}
H^{R}= & \lim _{\epsilon \rightarrow 0} H_{\epsilon}^{R}=\frac{\Delta}{\left(r^{2}+a^{2}\right)^{\frac{3}{2}}} \\
& \times \frac{1}{\sqrt{2 \pi}} \int_{\mathbb{S}^{2}} \Sigma e^{-i m \phi} S_{m \ell}(a \omega)\left(-2 \sqrt{-g^{t t}} \Psi_{1}-i \omega g^{t t} \Psi_{0}\right) \mathrm{d} \sigma_{\mathbb{S}^{2}} \\
& +\frac{\Delta}{\left(r^{2}+a^{2}\right)^{\frac{3}{2}}} \frac{e^{-i\left(\omega-\omega_{+} m\right) r^{*}}}{\sqrt{2 \pi}} \\
& \times \int_{R-1}^{R} \int_{\mathbb{S}^{2}} \Sigma F_{R}\left(v, r, \theta, \tilde{\phi}_{+}\right) e^{i \omega v} e^{-i m \tilde{\phi}_{+}} S_{m \ell}(a \omega) \mathrm{d} \sigma_{\mathbb{S}^{2}} \mathrm{~d} v
\end{aligned}
$$

pointwise.

Now, to show that $a_{\epsilon, \mathcal{H}}^{R} \rightarrow a_{\mathcal{H}}^{R}$ it suffices to show

$$
\int_{-\infty}^{\frac{\pi}{2}} u_{\infty} \frac{\Delta}{\left(r^{2}+a^{2}\right)^{\frac{3}{2}}} \widehat{\Sigma F_{\epsilon}} \mathrm{d} y \rightarrow \int_{-\infty}^{\frac{\pi}{2}} u_{\infty} \frac{\Delta}{\left(r^{2}+a^{2}\right)^{\frac{3}{2}}} \widehat{\Sigma F_{0}} \mathrm{~d} y
$$

pointwise as $\epsilon \rightarrow 0$. Again, recall from Definition 6.1 that our initial data are compactly supported in $K$. Thus, by finite speed of propagation we have that $r^{*} \mapsto F_{\epsilon}\left(r^{*}\right)$ is compactly supported (uniformly in the other coordinates) in an open neighborhood $K^{o} \supset K$ of $K$ for all $0<\epsilon<\epsilon_{0}$ sufficiently small. Note that $K^{o} \backslash K$ can be made arbitrarily small by choosing $\epsilon_{0}>0$ sufficiently small. Further, we show below that $\sup _{0<\epsilon<\epsilon_{0}} \sup _{r^{*}}\left|\widehat{\Sigma F_{\epsilon}}\right|<\infty$ so we can interchange the integral with the limit $\epsilon \rightarrow 0$ in (7.42).

We will now justify $\sup _{0<\epsilon<\epsilon_{0}} \sup _{r^{*}}\left|\widehat{\sum F_{\epsilon}}\right|<\infty$. Indeed, using that $\partial_{t}(\chi(t / \epsilon))$ is only supported in $[0, \epsilon]$ and integrating by parts we have

$$
\begin{aligned}
\left|\widehat{\Sigma F_{\epsilon}}\right| \lesssim & \mid \int_{\mathbb{S}^{2}} \int_{0}^{\epsilon} e^{-i m \phi} S_{m \ell}(a \omega, \cos \theta) e^{i \omega t}\left(-2 \partial_{t}(\chi(t / \epsilon)) \sqrt{-g^{t t}} n_{\Sigma_{t}} \psi\right. \\
& \left.+\psi g^{t t} \partial_{t}^{2}(\chi(t / \epsilon))\right) \mathrm{d} \sigma_{\mathbb{S}^{2}} \mathrm{~d} t \mid
\end{aligned}
$$




$$
\begin{aligned}
\lesssim & \mid \int_{\mathbb{S}^{2}} \int_{0}^{\epsilon} e^{-i m \phi} S_{m \ell}(a \omega, \cos \theta)\left(2 \chi(t / \epsilon) \sqrt{-g^{t t}} \partial_{t}\left(e^{i \omega t} n_{\Sigma_{t}} \psi\right)\right. \\
& \left.+g^{t t} \partial_{t}^{2}\left(e^{i \omega t} \psi\right) \chi(t / \epsilon)\right) \mathrm{d} \sigma_{\mathbb{S}^{2}} \mathrm{~d} t \mid \\
& +\mid \int_{\mathbb{S}^{2}} e^{-i m \phi} S_{m \ell}(a \omega, \cos \theta)\left(2 e^{i \omega \epsilon} \sqrt{-g^{t t}} n_{\Sigma_{t=\epsilon}} \psi(t=\epsilon)\right. \\
& \left.+\left(\partial_{t}\left(e^{i \omega t} \psi g^{t t}\right)\right)(t=\epsilon) \chi(t / \epsilon)\right) \mathrm{d} \sigma_{\mathbb{S}^{2}} \mid
\end{aligned}
$$

Since $\psi$ and all its derivatives are uniformly bounded and moreover are supported in $K^{o}$ in the $r^{*}$ coordinate we obtain $\sup _{0<\epsilon<\epsilon} \sup _{r^{*}}\left|\widehat{\Sigma F_{\epsilon}}\right|<\infty$ for $\epsilon_{0}>0$ sufficiently small.

Next, we will show that $H^{R} \rightarrow H$ as $R \rightarrow \infty$. As $\psi$ and its derivatives decay pointwise at a logarithmic rate (see Theorem 2), we obtain

$$
\sup _{r \in\left(r_{+}, \infty\right), \theta, \tilde{\phi}_{+} \in \mathbb{S}^{2}}\left|F_{r}\right|\left(v, r, \theta, \tilde{\phi}_{+}\right) \rightarrow 0
$$

as $R \rightarrow \infty$. Thus, we have

$$
\begin{aligned}
& \left|\int_{R-1}^{R} \int_{\mathbb{S}^{2}} \Sigma F_{R}\left(v, r, \theta, \tilde{\phi}_{+}\right) e^{i \omega v} e^{-i m \tilde{\phi}_{+}} S_{m \ell}(a \omega) \mathrm{d} \sigma_{\mathbb{S}^{2}}\right| \\
& \quad \lesssim \sup _{v \in(R-1, R) r \in\left(r_{+}, \infty\right), \theta, \tilde{\phi}_{+} \in \mathbb{S}^{2}}\left|F_{r}\right|\left(v, r, \theta, \tilde{\phi}_{+}\right) \rightarrow 0
\end{aligned}
$$

pointwise as $R \rightarrow \infty$. This shows $H^{R} \rightarrow H$ pointwise.

Finally, to show that $a_{\mathcal{H}}^{R} \rightarrow a_{\mathcal{H}}$ as $R \rightarrow \infty$, we estimate

$$
\begin{aligned}
& \mid \int_{-\infty}^{\frac{\pi}{2}} u_{\infty} \frac{\Delta}{\left(r^{2}+a^{2}\right)^{\frac{3}{2}}} \frac{e^{-i(\omega-\omega+m) r^{*}}}{\sqrt{2 \pi}} \\
& \quad \times\left.\int_{R}^{R+1} \int_{\mathbb{S}^{2}} \Sigma F_{R}\left(v, r, \theta, \tilde{\phi}_{+}\right) e^{i \omega v} e^{-i m \tilde{\phi}_{+}} S_{m \ell}(a \omega) \mathrm{d} \sigma_{\mathbb{S}^{2}} \mathrm{~d} v \mathrm{~d} r^{*}\right|^{2} \\
& \lesssim \int_{-\infty}^{\frac{\pi}{2}} \int_{\mathbb{S}^{2}}\left|S_{m \ell}(a \omega)\right|^{2}\left|u_{\infty}\right|^{2} \frac{1}{r^{2}} \frac{\Delta}{r^{2}+a^{2}} \mathrm{~d} \sigma_{\mathbb{S}^{2}} \mathrm{~d} r^{*} \\
& \quad \times \sup _{v \in(R, R+1)} \int_{r_{+}}^{\infty} \int_{\mathbb{S}^{2}} \Sigma^{2}\left|F_{R}\right|^{2} \frac{1}{r^{2}+a^{2}} r^{2} \frac{\Delta}{r^{2}+a^{2}} \mathrm{~d} \sigma_{\mathbb{S}^{2}} \mathrm{~d} r^{*} \\
& \lesssim \int_{-\infty}^{\frac{\pi}{2}}\left|u_{\infty}\right|^{2} \frac{1}{r^{2}} \frac{\Delta}{r^{2}+a^{2}} \mathrm{~d} r^{*} \\
& \quad \times \sup _{v \in(R, R+1)} \int_{r_{+}}^{\infty} \int_{\mathbb{S}^{2}} \Sigma^{2}\left|F_{R}\right|^{2} \frac{1}{r^{2}+a^{2}} r^{2} \mathrm{~d} \sigma_{\mathbb{S}^{2}} \mathrm{~d} r
\end{aligned}
$$




$$
\begin{aligned}
& \lesssim \int_{-\infty}^{\frac{\pi}{2}} C_{m \ell \omega}^{2}\left|r^{*}\right|^{2} \frac{1}{r^{2}} \frac{\Delta}{r^{2}+a^{2}} \mathrm{~d} r^{*} \sup _{v \in(R, R+1)} \int_{r_{+}}^{\infty} \int_{\mathbb{S}^{2}} r^{2}\left|F_{R}\right|^{2} r^{2} \mathrm{~d} \sigma_{\mathbb{S}^{2}} \mathrm{~d} r \\
& \lesssim C_{m \ell \omega}^{2} \sup _{v \in(R, R+1)} \int_{r_{+}}^{\infty} \int_{\mathbb{S}^{2}} e_{1}[\psi] r^{2} \mathrm{~d} \sigma_{\mathbb{S}^{2}} \mathrm{~d} r \rightarrow 0
\end{aligned}
$$

as $R \rightarrow \infty$. Here, we have used (7.29) again. Hence, $a_{\mathcal{H}}^{R} \rightarrow a_{\mathcal{H}}$ as $R \rightarrow \infty$ pointwise for each $\omega, m, \ell$.

\subsection{Representation formula for $\psi$ at the event horizon}

In what follows we will prove a representation formula of the truncated solution $\psi^{R}$ along the event horizon in terms of the initial data and an error term which vanishes in the limit $R \rightarrow \infty$. More precisely, we will represent $\psi^{R}$ through $a_{\mathcal{H}}^{R}$ which is defined in (7.34). Note that in the limit $R \rightarrow \infty$, we have $a_{\mathcal{H}}^{R} \rightarrow a_{\mathcal{H}}$ which in turn only depends on the initial data (see Definition 6.3).

Proposition 7.1 Let $a_{\mathcal{H}}^{R}$ be as defined in (7.34). Then, on the event horizon $\mathcal{H}_{R}$ we have

$\psi^{R}\left(v, r_{+}, \theta, \tilde{\phi}_{+}\right)=\frac{1}{\sqrt{2 \pi\left(r_{+}^{2}+a^{2}\right)}} \sum_{m \ell} \int_{\mathbb{R}} a_{\mathcal{H}}^{R} S_{m \ell}(a \omega, \cos \theta) e^{i m \tilde{\phi}_{+}} e^{-i \omega v} \mathrm{~d} \omega$,

in $L^{2}\left(\mathbb{R}_{v} \times \mathbb{S}^{2}\right)$. Moreover,

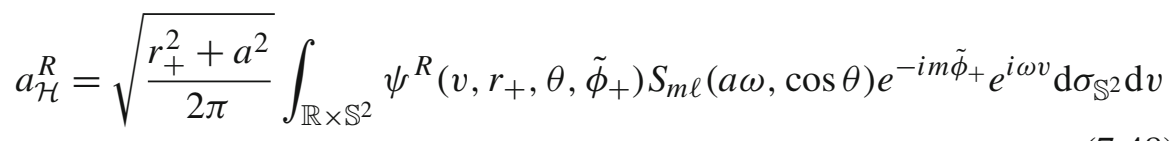

pointwise and in $L^{2}\left(\mathbb{R}_{\omega} \times \mathbb{Z}_{m} \times \mathbb{Z}_{\ell \geq|m|}\right)$.

Proof We have

$$
\begin{aligned}
\psi_{\epsilon}^{R}\left(v, r, \theta, \tilde{\phi}_{+}\right)= & \frac{1}{\sqrt{2 \pi\left(r^{2}+a^{2}\right)}} \\
& \times \sum_{m \ell} \int_{\mathbb{R}} e^{i\left(\omega-\omega_{+} m\right) r^{*}} u_{\epsilon}^{R} S_{m \ell}(a \omega, \cos \theta) e^{i m \tilde{\phi}_{+}} e^{-i \omega v} \mathrm{~d} \omega
\end{aligned}
$$


and

$$
\begin{aligned}
& e^{i\left(\omega-\omega_{+} m\right) r^{*} u_{\epsilon}^{R}} \\
& \quad=\sqrt{\frac{r^{2}+a^{2}}{2 \pi}} \int_{\mathbb{R} \times \mathbb{S}^{2}} \psi_{\epsilon}^{R}\left(v, r, \theta, \tilde{\phi}_{+}\right) S_{m \ell}(a \omega, \cos \theta) e^{-i m \tilde{\phi}_{+}} e^{i \omega v} \mathrm{~d} \sigma_{\mathbb{S}^{2}} \mathrm{~d} v
\end{aligned}
$$

for $r_{+}<r<r_{+}+\eta$. Now, since $\psi_{\epsilon}^{R}$ is compactly supported in $v$ uniformly as $r_{*} \rightarrow-\infty$, we can interchange the limit $r^{*} \rightarrow-\infty$ with the integral over $v$. Thus, sending $r \rightarrow r_{+}\left(r^{*} \rightarrow-\infty\right)$ in (7.50) yields in view of Lemma 7.2 that

$a_{\epsilon, \mathcal{H}}^{R}=\sqrt{\frac{r_{+}^{2}+a^{2}}{2 \pi}} \int_{\mathbb{R} \times \mathbb{S}^{2}} \psi_{\epsilon}^{R}\left(v, r_{+}, \theta, \tilde{\phi}_{+}\right) S_{m \ell}(a \omega, \cos \theta) e^{-i m \tilde{\phi}_{+}} e^{i \omega v} \mathrm{~d} \sigma_{\mathbb{S}^{2}} \mathrm{~d} v$,

where $a_{\epsilon, \mathcal{H}}^{R}$ is defined in (7.28). Now we will perform the limit $\epsilon \rightarrow 0$ on both sides of (7.51) independently. First, from Lemma 7.3 we have that

$$
a_{\epsilon, \mathcal{H}}^{R} \rightarrow a_{\mathcal{H}}^{R}=\frac{1}{\mathfrak{W}\left[u_{\mathcal{H}^{+}}, u_{\infty}\right]} \int_{-\infty}^{\frac{\pi}{2}} u_{\infty} H^{R} \mathrm{~d} y
$$

as $\epsilon \rightarrow 0$ pointwise. Moreover, $\psi_{\epsilon}^{R}$ has compact support on $\mathbb{R}_{v}$ uniformly as $\epsilon \rightarrow 0$ and $\psi_{\epsilon}^{R} \rightarrow \psi^{R}$ pointwise and in $L^{2}\left(\mathbb{R}_{v} \times \mathbb{S}^{2}\right)$ as $\epsilon \rightarrow 0$. Thus, the right hand side of (7.51) converges pointwise and due to Plancherel also in $L^{2}\left(\mathbb{R}_{\omega} \times \mathbb{Z}_{m} \times \mathbb{Z}_{\ell \geq|m|}\right)$ as $\epsilon \rightarrow 0$. Hence, $a_{\epsilon, \mathcal{H}}^{R} \rightarrow a_{\mathcal{H}}^{R}$ also holds in $L^{2}\left(\mathbb{R}_{\omega} \times \mathbb{Z}_{m} \times \mathbb{Z}_{\ell \geq|m|}\right)$ and we conclude

$$
a_{\mathcal{H}}^{R}=\sqrt{\frac{r_{+}^{2}+a^{2}}{2 \pi}} \int_{\mathbb{R} \times \mathbb{S}^{2}} \psi^{R}\left(v, r_{+}, \theta, \tilde{\phi}_{+}\right) S_{m \ell}(a \omega, \cos \theta) e^{-i m \tilde{\phi}_{+}} e^{i \omega v} \mathrm{~d} \sigma_{\mathbb{S}^{2}} \mathrm{~d} v
$$

which holds pointwise and in $L^{2}\left(\mathbb{R}_{\omega} \times \mathbb{Z}_{m} \times \mathbb{Z}_{\ell \geq|m|}\right)$. And by Plancherel we also have

$$
\psi^{R}\left(v, r_{+}, \theta, \tilde{\phi}_{+}\right)=\frac{1}{\sqrt{2 \pi\left(r_{+}^{2}+a^{2}\right)}} \sum_{m \ell} \int_{\mathbb{R}} a_{\mathcal{H}}^{R} S_{m \ell}(a \omega, \cos \theta) e^{i m \tilde{\phi}_{+}} e^{-i \omega v} \mathrm{~d} \omega
$$

in $L^{2}\left(\mathbb{R}_{v} \times \mathbb{S}^{2}\right)$ 


\section{Interior analysis: Estimates on radial o.d.e. and interior scattering poles}

Having established the behavior of our solution $\psi$ on the exterior $\mathcal{R}$, we will now consider the interior region $\mathcal{B}$ characterized by $r \in\left(r_{-}, r_{+}\right)$. We will first consider the interior radial o.d.e. and prove a suitable representation formula on the interior. We also recall that in the interior region the tortoise coordinate is defined in (2.18) as

$$
\frac{\mathrm{d} r^{*}}{\mathrm{~d} r}=\frac{r^{2}+a^{2}}{\Delta}
$$

where $r^{*}\left(\frac{r_{+}+r_{-}}{2}\right)=0$ and that $\Delta<0$ in the whole interior region.

Remark 8.1 As our initial data are only supported on azimuthal modes $m$ which are large and positive, we only need to consider $m$ sufficiently large.

\subsection{Radial o.d.e. on the interior: fixed frequency scattering}

We recall the radial o.d.e. (2.43) and write it in the interior $r_{-}<r<r_{+}$as

$$
-u^{\prime \prime}+\left(\frac{\Delta L}{\left(r^{2}+a^{2}\right)^{2}}-\left(m \omega_{r}-\omega\right)^{2}+V_{1}\right) u=0,
$$

where

$$
L:=\lambda_{m \ell}+a^{2} \omega^{2}-2 m \omega a \Xi
$$

and $V_{1}$ is defined in (2.45). Note that $L \geq 0$ follows from [63, Lemma 5.4]. Also note that $V_{1}=O(|\Delta|)$ uniformly for $r^{*} \in(-\infty, \infty)$. We will treat $V_{1}$ as a perturbation and recall that the high-frequency part of the potential is given by

$$
V_{\sharp}:=\frac{\Delta L}{\left(r^{2}+a^{2}\right)^{2}}-\left(m \omega_{r}-\omega\right)^{2} .
$$

(Note that $V_{\text {main }}=\frac{V_{\sharp}\left(\omega=\omega_{-} m\right)}{m^{2}}$.) Analogously to Definition 4.2, we define fundamental pairs of solutions to the radial o.d.e. corresponding to the event and Cauchy horizon, respectively.

Definition 8.1 We define solutions $u_{\mathcal{H}_{R}}, u_{\mathcal{H}_{L}}$ to (8.2) in the interior through the condition

$$
u_{\mathcal{H}_{R}}=e^{-i\left(\omega-\omega_{+} m\right) r^{*}}+O_{\omega, m, \ell}(\Delta),
$$




$$
u_{\mathcal{H}_{L}}=e^{i\left(\omega-\omega_{+} m\right) r^{*}}+O_{\omega, m, \ell}(\Delta)
$$

as $r^{*} \rightarrow-\infty$. For $\omega \neq \omega_{+} m$, they form a fundamental pair. For $\omega=\omega_{+} m$ the solutions $u_{\mathcal{H}_{L}}$ and $u_{\mathcal{H}_{R}}$ are linearly dependent.

Analogously, we define

$$
\begin{aligned}
& u_{\mathcal{C H}_{L}}=e^{-i(\omega-\omega-m) r^{*}}+O_{\omega, m, \ell}(\Delta), \\
& u_{\mathcal{C H}}=e^{i\left(\omega-\omega_{-} m\right) r^{*}}+O_{\omega, m, \ell}(\Delta)
\end{aligned}
$$

as $r^{*} \rightarrow+\infty$. For $\omega \neq \omega_{-} m$, they form a fundamental pair. For $\omega=\omega_{-} m$ the solutions $u_{\mathcal{C H}}$ and $u_{\mathcal{C H}}$ are linearly dependent.

Remark 8.2 As in Remark 4.1 we can equivalently define $u_{\mathcal{H}_{R}}$ (and analogously $u_{\mathcal{H}_{L}}, u_{\mathcal{C H}_{L}}, u_{\mathcal{C H}_{R}}$ ) as the unique solution to the Volterra integral equation

$$
\begin{aligned}
u_{\mathcal{H}_{R}}\left(r^{*}\right)= & e^{-i\left(\omega-\omega_{+} m\right) r^{*}} \\
& +\int_{-\infty}^{r^{*}} \frac{\sin \left(\left(\omega-\omega_{+} m\right)\left(r^{*}-y\right)\right)}{\omega-\omega_{+} m}\left(V_{\sharp}(y)+V_{1}(y)\right. \\
& \left.+\left(\omega-\omega_{+} m\right)^{2}\right) u_{\mathcal{H}_{R}}(y) \mathrm{d} y .
\end{aligned}
$$

We moreover define reflection and transmission coefficients.

Definition 8.2 For $\omega \neq \omega_{-} m$ define the transmission coefficient $\mathfrak{T}=$ $\mathfrak{T}(\omega, m, \ell)$ and the reflection coefficient $\mathfrak{R}=\mathfrak{R}(\omega, m, \ell)$ as the unique coefficients such that

$$
\begin{aligned}
u_{\mathcal{H}_{R}}\left(r^{*}, \omega, m, \ell\right)= & \mathfrak{T}(\omega, m, \ell) u_{\mathcal{C H}_{L}}\left(r^{*}, \omega, m, \ell\right) \\
& +\mathfrak{R}(\omega, m, \ell) u_{\mathcal{C H}_{R}}\left(r^{*}, \omega, m, \ell\right) .
\end{aligned}
$$

Equivalently, we have

$$
\begin{aligned}
\mathfrak{T}(\omega, m, \ell) & =\frac{\mathfrak{W}\left[u_{\mathcal{H}_{R}}, u_{\mathcal{C H}_{R}}\right](\omega, m, \ell)}{\mathfrak{W}\left[u_{\mathcal{C H}_{L}}, u_{\mathcal{C H}_{R}}\right](\omega, m, \ell)}=\frac{\mathfrak{W}\left[u_{\mathcal{H}_{R}}, u_{\mathcal{C H}}\right](\omega, m, \ell)}{2 i(\omega-\omega-m)}, \\
\mathfrak{R}(\omega, m, \ell) & =\frac{\mathfrak{W}\left[u_{\mathcal{H}_{R}}, u_{\mathcal{C H}_{L}}\right](\omega, m, \ell)}{\mathfrak{W}\left[u_{\mathcal{C H}_{R}}, u_{\mathcal{C H}}\right](\omega, m, \ell)}=\frac{\mathfrak{W}\left[u_{\mathcal{H}_{R}}, u_{\mathcal{C H}}\right](\omega, m, \ell)}{-2 i\left(\omega-\omega_{-} m\right)} .
\end{aligned}
$$

Further, we define the renormalized transmission and reflection coefficient

$$
\mathfrak{t}(\omega, m, \ell):=\left(\omega-\omega_{-} m\right) \mathfrak{T}(\omega, m, \ell)=\frac{1}{2 i} \mathfrak{W}\left[u_{\mathcal{H}_{R}}, u_{\mathcal{C H}}\right](\omega, m, \ell),
$$




$$
\mathfrak{r}(\omega, m, \ell):=\left(\omega-\omega_{-} m\right) \mathfrak{R}(\omega, m, \ell)=-\frac{1}{2 i} \mathfrak{W}\left[u_{\mathcal{H}_{R}}, u_{\mathcal{C H}}\right](\omega, m, \ell)
$$

which satisfy

$$
\mathfrak{t}^{\omega_{-}}(m, \ell)=-\mathfrak{r}^{\omega_{-}}(m, \ell),
$$

where

$$
\mathfrak{t}^{\omega_{-}}(m, \ell):=\mathfrak{t}\left(\omega=\omega_{-} m, m, \ell\right) \quad \text { and } \quad \mathfrak{r}^{\omega_{-}}(m, \ell):=\mathfrak{r}\left(\omega=\omega_{-} m, m, \ell\right)
$$

Lemma 8.1 The transmission and reflection coefficients satisfy the Wronskian identity

$$
|\mathfrak{T}(\omega, m, \ell)|^{2}=|\mathfrak{R}(\omega, m, \ell)|^{2}+\frac{\omega-\omega_{+} m}{\omega-\omega_{-} m}
$$

for $\omega \in \mathbb{R} \backslash\left\{\omega_{-} m\right\}$.

Proof We decompose

$$
u_{\mathcal{H}_{R}}=\mathfrak{T} u_{\mathcal{C H}}+\mathfrak{R} u_{\mathcal{C H}} \text {. }
$$

Since the potential of the o.d.e. (8.2) is real-valued we have that $u_{\mathcal{H}_{L}}=u_{\overline{\mathcal{H}}_{R}}$. Thus,

$$
u_{\mathcal{H}_{L}}=u_{\overline{\mathcal{H}}}=\overline{\mathfrak{T}} u_{\overline{\mathcal{C H}}}+\overline{\mathfrak{R}} u_{\overline{\mathcal{C H}}}=\overline{\mathfrak{T}} u_{\mathcal{C H}}+\bar{\Re} u_{\mathcal{C H}}
$$

Now, using $\mathfrak{W}\left(u_{\mathcal{H}_{R}}, u_{\mathcal{H}_{L}}\right)=2 i\left(\omega-\omega_{+} m\right),(8.18)$ and (8.19) yields the result.

We begin by showing $L^{\infty}$ estimates for the solutions defined in (8.1). To do so we will consider the cases $\left|\omega-\omega_{r} m\right| \geq \epsilon_{\text {cut }} m$ for all $r \in\left[r_{-}, r_{+}\right]$. Note that $\epsilon_{\text {cut }}>0$ will be fixed in Lemma 8.3 only depending on the black hole parameters.

Lemma 8.2 Assume that $\left|\omega-\omega_{r} m\right| \geq \epsilon_{\text {cut }} m$ for all $r \in\left[r_{-}, r_{+}\right]$and for some $\epsilon_{\text {cut }}>0$. Then

$$
\begin{aligned}
\left\|u_{\mathcal{H}_{R}}\right\|_{L^{\infty}(\mathbb{R})} \lesssim 1, \quad\left\|u_{\mathcal{H}_{R}}{ }^{\prime}\right\|_{L^{\infty}(\mathbb{R})} \lesssim|\omega|+|m|+L^{\frac{1}{2}} \\
\left\|u_{\mathcal{C} \mathcal{H}_{L}}\right\|_{L^{\infty}(\mathbb{R})} \lesssim 1, \quad\left\|u_{\mathcal{C H}_{L}}{ }^{\prime}\right\|_{L^{\infty}(\mathbb{R})} \lesssim|\omega|+|m|+L^{\frac{1}{2}}, \\
\left\|u_{\mathcal{C} \mathcal{H}_{R}}\right\|_{L^{\infty}(\mathbb{R})} \lesssim 1, \quad\left\|u_{\mathcal{C} \mathcal{H}_{R}}{ }^{\prime}\right\|_{L^{\infty}(\mathbb{R})} \lesssim|\omega|+|m|+L^{\frac{1}{2}} .
\end{aligned}
$$


Proof We first consider the case that $\omega-\omega_{r} m \geq \epsilon_{\text {cut }} m$ for all $r \in\left[r_{-}, r_{+}\right]$.

First, we also assume $L^{\frac{1}{2}} \leq|\omega|+|m|$. Then, in view of the assumptions, the principal part of the potential $V_{\sharp}$ satisfies

$$
-V_{\sharp} \gtrsim m^{2}+\omega^{2} \quad \text { and } \quad\left|\frac{V_{\sharp}^{\prime}}{V_{\sharp}}\right|,\left|\frac{V_{\sharp}^{\prime \prime}}{V_{\sharp}}\right| \lesssim|\Delta|
$$

and the error term satisfies $\left|V_{1}\right| \lesssim|\Delta|$. Thus, the error control function

$$
F_{u_{\mathcal{H}_{R 1}}}\left(r^{*}\right):=\int_{-\infty}^{r^{*}} \frac{1}{\left|V_{\sharp}\right|^{\frac{1}{4}}} \frac{\mathrm{d}^{2}}{\mathrm{~d} y^{2}}\left(\left|V_{\sharp}\right|^{-\frac{1}{4}}\right)-\frac{V_{1}}{\left|V_{\sharp}\right|^{\frac{1}{2}}} \mathrm{~d} y
$$

satisfies $\mathcal{V}_{-\infty, \infty}\left(F_{u_{\mathcal{H}_{R}}}\right) \lesssim \frac{1}{m}$

In the case $L^{\frac{1}{2}} \geq|\omega|+|m|$ we have

$$
-V_{\sharp} \gtrsim|\Delta| L+m^{2}+\omega^{2} \quad \text { and } \quad\left|\frac{V_{\sharp}^{\prime}}{V_{\sharp}}\right|,\left|\frac{V_{\sharp}^{\prime \prime}}{V_{\sharp}}\right| \lesssim \frac{|\Delta| L}{|\Delta| L+m^{2}+\omega^{2}}
$$

uniformly for $r^{*} \in \mathbb{R}$. Making use of (8.25), we estimate the total variation of $F_{u \mathcal{H}_{R 1}}$ in this frequency range as

$$
\begin{aligned}
& \mathcal{V}_{-\infty, \infty}\left(F_{u_{\mathcal{H}_{R} 1}}\right) \lesssim \int_{\mathbb{R}} \frac{\left|V_{\sharp}^{\prime}\right|^{2}}{\left|V_{\sharp}\right|^{\frac{5}{2}}}+\frac{\left|V_{\sharp}^{\prime \prime}\right|}{\left|V_{\sharp}\right|^{\frac{3}{2}}}+\frac{\left|V_{1}\right|}{\left|V_{\sharp}\right|^{\frac{1}{2}}} \mathrm{~d} y \\
& \lesssim \int_{\mathbb{R}} \frac{|\Delta| L}{\left(|\Delta| L+m^{2}+|\omega|^{2}\right)^{\frac{3}{2}}} \mathrm{~d} y+\frac{1}{m} \\
& \lesssim \int_{r_{-}}^{r_{+}} \frac{L}{\left(\left|r-r_{-}\right|\left|r-r_{+}\right| L+m^{2}+|\omega|^{2}\right)^{\frac{3}{2}}} \mathrm{~d} r+\frac{1}{m} \\
& \lesssim L^{-\frac{1}{2}} \int_{r_{-}}^{r_{+}} \frac{1}{\left(\left|r-r_{-}\right|\left|r-r_{+}\right|+m^{2} L^{-1}+|\omega|^{2} L^{-1}\right)^{\frac{3}{2}}} \mathrm{~d} r \\
& +\frac{1}{m} \lesssim L^{-\frac{1}{2}}\left(m^{2} L^{-1}+\omega^{2} L^{-1}\right)^{-\frac{1}{2}}+\frac{1}{m} \lesssim \frac{1}{m} \text {. }
\end{aligned}
$$

Here, we used the definition of $r^{*}$ in (8.1) as well as $|\Delta| \sim\left|r-r_{-}\right|\left|r-r_{+}\right|$ uniformly for $r \in\left(r_{-}, r_{+}\right)$.

Thus, in both of the above cases, $L^{\frac{1}{2}} \leq|\omega|+|m|$ and $L^{\frac{1}{2}} \geq|\omega|+|m|$, the above allows us to apply standard estimates on WKB approximation such as 
[94, Chapter 6, Theorem 2.2] and deduce that

$$
u_{\mathcal{H}_{R}}=A_{u_{\mathcal{H}_{R}}} \frac{\left|V_{\sharp}(-\infty)\right|^{\frac{1}{4}}}{\left|V_{\sharp}\left(r^{*}\right)\right|^{\frac{1}{4}}} \exp \left(-i \int_{0}^{r^{*}}\left|V_{\sharp}(y)\right|^{\frac{1}{2}} \mathrm{~d} y\right)\left(1+\epsilon_{u_{\mathcal{H}_{R}}}\left(r^{*}\right)\right),
$$

for some $A_{u_{\mathcal{H}_{R}}}$ with $\left|A_{u_{\mathcal{H}_{R}}}\right|=1$. Moreover,

$$
\begin{aligned}
& \sup _{r^{*} \in \mathbb{R}}\left|\epsilon_{u_{\mathcal{H}_{R}}}\left(r^{*}\right)\right| \lesssim \frac{1}{m}, \sup _{r^{*} \in \mathbb{R}}\left|\frac{\epsilon_{u_{\mathcal{H}_{R}}}^{\prime}\left(r^{*}\right)}{\left|V_{\sharp}\right|^{\frac{1}{2}}}\right| \lesssim \frac{1}{m} \quad \text { and } \\
& \epsilon_{u_{\mathcal{H}_{R}}}(-\infty)=\epsilon_{u_{\mathcal{H}_{R}}^{\prime}}^{\prime}(-\infty)=0 .
\end{aligned}
$$

This shows that

$$
\left\|u_{\mathcal{H}_{R}}\right\|_{L^{\infty}(\mathbb{R})} \lesssim 1 \quad \text { and } \quad\left\|u_{\mathcal{H}_{R}}{ }^{\prime}\right\|_{L^{\infty}(\mathbb{R})} \lesssim\left\|\left|V_{\sharp}\right|^{\frac{1}{2}}\right\|_{L^{\infty}(\mathbb{R})} \lesssim|\omega|+|m|+L^{\frac{1}{2}}
$$

Similarly, we show that the above holds for $\omega_{r} m-\omega \geq \epsilon_{\text {cut }} m$. This shows (8.20). The bounds (8.21) and (8.22) are shown completely analogously and their proofs are omitted.

Lemma 8.3 There exists a constant $\epsilon_{\mathrm{cut}}>0$ such that the following holds true. Assume that $\left|\omega-\omega_{r} m\right| \leq \epsilon_{\mathrm{cut}} m$ for some $r \in\left[r_{-}, r_{+}\right]$, then $L \gtrsim m^{2}$.

Proof Note that $L$ is larger than the lowest eigenvalue of the operator $P(a \omega)+$ $a^{2} \omega^{2}-2 a \Xi \omega m$, where $P$ is as in (2.41). Since

$$
\begin{aligned}
P(a \omega)+a^{2} \omega^{2}-2 a \Xi \omega m= & -\frac{1}{\sin \theta} \partial_{\theta}\left(\Delta_{\theta} \sin \theta \partial_{\theta} \cdot\right) \\
& +\frac{1}{\Delta_{\theta}}\left(m \frac{\Xi}{\sin \theta}-a \omega \sin \theta\right)^{2}+2 \frac{a^{2}}{l^{2}} \sin ^{2} \theta,
\end{aligned}
$$

it suffices to show that the second term is bounded from below by $O\left(\mathrm{~m}^{2}\right)$. To do so, let $r \in\left[r_{-}, r_{+}\right]$such that $\left|\omega-\omega_{r} m\right| \leq \epsilon_{\text {cut }} m$. Then, in view of

$$
a \omega_{r}=\Xi \frac{a^{2}}{r^{2}+a^{2}},
$$


we conclude

$$
\begin{aligned}
\left(m \frac{\Xi}{\sin \theta}-a \omega \sin \theta\right)^{2} & =\left(m \frac{\Xi}{\sin \theta}-a \omega_{r} m \sin \theta+a\left(\omega_{r} m-\omega\right) \sin \theta\right)^{2} \\
& \geq \frac{m^{2} \Xi^{2}}{\sin ^{2} \theta}\left(1-\frac{a^{2}}{a^{2}+r^{2}} \sin ^{2} \theta-\left|\frac{a}{\Xi} \frac{\omega_{r} m-\omega}{m} \sin ^{2} \theta\right|\right)^{2} \gtrsim m^{2}
\end{aligned}
$$

for sufficiently small $\epsilon_{\text {cut }}>0$.

In the rest of the section we will make use of

Definition 8.3 For all frequencies $\omega, m, \ell$ we define

$$
\begin{aligned}
& u_{\tilde{\mathcal{H}}_{R}}:=e^{i\left(\omega-\omega_{-} m\right) r^{*}} u_{\mathcal{H}_{R}}, \\
& u_{\tilde{\mathcal{H}}_{L}}:=e^{-i\left(\omega-\omega_{-} m\right) r^{*}} u_{\mathcal{H}_{L}}, \\
& u_{\tilde{\mathcal{H}}_{R}}:=e^{-i\left(\omega-\omega_{-} m\right) r^{*}} u_{\mathcal{C H}_{R}}, \\
& u_{\tilde{\mathcal{C H}}_{L}}:=e^{i\left(\omega-\omega_{-} m\right) r^{*}} u_{\mathcal{C H}_{L}} .
\end{aligned}
$$

Lemma 8.4 Assume that $\left|\omega-\omega_{r} m\right| \leq \epsilon_{\mathrm{cut}} m$ for some $r \in\left[r_{-}, r_{+}\right]$and assume that $m \in \mathbb{N}$ is sufficiently large. Define

$$
\begin{aligned}
& R_{1}:=-\frac{1}{2 \kappa_{+}} \log (L), \\
& R_{2}:=\frac{1}{2\left|\kappa_{-}\right|} \log (L) .
\end{aligned}
$$

Then

$$
\begin{aligned}
\left\|u_{\mathcal{H}_{R}}\right\|_{L^{\infty}{ }_{\left(-\infty, R_{2}\right]}} \lesssim 1,\left\|u_{\mathcal{H}_{R}}{ }^{\prime}\right\|_{L^{\infty}{ }_{\left(-\infty, R_{2}\right]} \lesssim|\omega|+|m|+L^{\frac{1}{2}}} \\
\left\|u_{\mathcal{C H}_{L}}\right\|_{L^{\infty}\left[R_{1}, \infty\right)} \lesssim 1,\left\|u_{\mathcal{C H}_{L}}{ }^{\prime}\right\|_{L^{\infty}\left[R_{1}, \infty\right)} \lesssim|\omega|+|m|+L^{\frac{1}{2}}, \\
\left\|u_{\mathcal{C} \mathcal{H}_{R}}\right\|_{L^{\infty}\left[R_{1}, \infty\right)} \lesssim 1,\left\|u_{\mathcal{C} \mathcal{H}_{R}}{ }^{\prime}\right\|_{L^{\infty}\left[R_{1}, \infty\right)} \lesssim|\omega|+|m|+L^{\frac{1}{2}},
\end{aligned}
$$

and

$$
\begin{aligned}
\left|\partial_{\omega} u_{\mathcal{H}_{R}}\right|\left(R_{1}\right) \lesssim \log (L),\left|\partial_{\omega} u_{\mathcal{H}_{R}}{ }^{\prime}\right|\left(R_{1}\right) \lesssim \log (L)(|\omega|+|m|), \\
\left|\partial_{\omega} u_{\mathcal{C H}}\right|\left(R_{2}\right) \lesssim \log (L),\left|\partial_{\omega} u_{\mathcal{C H}}{ }^{\prime}\right|\left(R_{2}\right) \lesssim \log (L)(|\omega|+|m|), \\
\left|\partial_{\omega} u_{\mathcal{C H}}\right|\left(R_{2}\right) \lesssim \log (L),\left|\partial_{\omega} u_{\mathcal{C H}}{ }^{\prime}\right|\left(R_{2}\right) \lesssim \log (L)(|\omega|+|m|) .
\end{aligned}
$$


Moreover,

$$
\begin{aligned}
& \left\|\partial_{\omega} u \tilde{\mathcal{H}}_{R}\right\|_{L^{\infty}\left(-\infty, R_{1}\right)} \lesssim 1,\left\|\partial_{\omega} u \tilde{\mathcal{H}}_{R}{ }^{\prime}\right\|_{L^{\infty}\left(-\infty, R_{1}\right)} \lesssim 1, \\
& \left\|\partial_{\omega} u \tilde{\mathcal{C}}_{R}\right\|_{L^{\infty}\left(R_{2}, \infty\right)} \lesssim 1,\left\|\partial_{\omega} u \tilde{\mathcal{C}}_{R}{ }^{\prime}\right\|_{L^{\infty}\left(R_{2}, \infty\right)} \lesssim 1 \text {, } \\
& \left\|\partial_{\omega} u_{\mathcal{C} \tilde{\mathcal{H}}_{L}}\right\|_{L^{\infty}\left(R_{2}, \infty\right)} \lesssim 1,\left\|\partial_{\omega} u \tilde{\mathcal{C H}}_{L}^{\prime}\right\|_{L^{\infty}\left(R_{2}, \infty\right)} \lesssim 1 .
\end{aligned}
$$

Proof From Lemma 8.3 we know that $L \gtrsim m^{2}$. Now, in view of Remark 8.2 we write $u_{\mathcal{H}}$ as the solution to the Volterra equation

$$
u_{\mathcal{H}_{R}}=e^{-i\left(\omega-\omega_{+} m\right) r^{*}}+\int_{-\infty}^{r^{*}} K\left(r^{*}, y\right)\left(1+R_{1}-y\right) \bar{V}(y) u_{\mathcal{H}_{R}}(y) \mathrm{d} y,
$$

where the kernel is given by

$$
K\left(r^{*}, y\right)=\frac{1}{1+R_{1}-y} \frac{\sin \left(\left(\omega-\omega_{+} m\right)\left(r^{*}-y\right)\right)}{\omega-\omega_{+} m}
$$

and $\bar{V}:=V_{\sharp}+V_{1}+\left(\omega-\omega_{+} m\right)^{2}$. For $y \in\left(-\infty, R_{1}\right)$, a direct computation shows

$$
\begin{gathered}
\left(1+R_{1}-y\right)|\bar{V}(y)| \lesssim\left(1+R_{1}-y\right) L e^{2 \kappa+y}, \\
\int_{-\infty}^{R_{1}}\left(1+R_{1}-y\right)|\bar{V}(y)| \mathrm{d} y \lesssim 1
\end{gathered}
$$

and

$$
\sup _{y \leq r^{*} \leq R_{1}}\left|K\left(r^{*}, y\right)\right| \lesssim 1
$$

Standard estimates on Volterra integral equations (apply [94, Chapter 6, Theorem 10.1] to the term $\left.u_{\mathcal{H}_{R}}-e^{-i\left(\omega-\omega_{+} m\right) r^{*}}\right)$ yield

$$
\begin{aligned}
\left\|u_{\mathcal{H}_{R}}\right\|_{L^{\infty}\left(-\infty, R_{1}\right)} & \lesssim 1 \\
\left\|u_{\mathcal{H}_{R}}{ }^{\prime}\right\|_{L^{\infty}\left(-\infty, R_{1}\right)} & \lesssim 1+\left|\omega-\omega_{+} m\right| \lesssim m \mid .
\end{aligned}
$$

Now, for the region $r^{*} \in\left[R_{1}, R_{2}\right]$ we approximate $u_{\mathcal{H}_{R}}$ with a WKB approximation. To do so we remark that for $r^{*} \in\left[R_{1}, R_{2}\right]$ we have

$$
-V_{\sharp} \gtrsim 1 \quad \text { and } \quad\left|\frac{V_{\sharp}^{\prime}}{V_{\sharp}}\right|,\left|\frac{V_{\sharp}^{\prime \prime}}{V_{\sharp}}\right| \lesssim|\Delta|
$$


and the error term satisfies $\left|V_{1}\right| \lesssim|\Delta|$. Thus, the error control function

$$
F_{u_{\mathcal{H}_{R 2}}}\left(r^{*}\right):=\int_{R_{1}}^{r^{*}} \frac{1}{\left|V_{\sharp}\right|^{\frac{1}{4}}} \frac{\mathrm{d}^{2}}{\mathrm{~d} y^{2}}\left(\left|V_{\sharp}\right|^{-\frac{1}{4}}\right)-\frac{V_{1}}{\left|V_{\sharp}\right|^{\frac{1}{2}}} \mathrm{~d} y
$$

is bounded as $\mathcal{V}_{R_{1}, R_{2}}\left(F_{u_{\mathcal{H}_{R 2}}}\right) \lesssim 1$. This allows us to apply [94, Chapter 6, Theorem 2.2] to deduce that

$$
\begin{aligned}
u_{\mathcal{H}_{R}}= & A_{u_{\mathcal{H}_{R}}} u_{\mathrm{WKB}_{A}}+B_{u_{\mathcal{H}}} u_{\mathrm{WKB}_{B}} \\
= & A_{u_{\mathcal{H}_{R}}} \frac{\left|V_{\sharp}\left(R_{1}\right)\right|^{\frac{1}{4}}}{\left|V_{\sharp}\left(r^{*}\right)\right|^{\frac{1}{4}}} \exp \left(-i \int_{R_{1}}^{r^{*}}\left|V_{\sharp}(y)\right|^{\frac{1}{2}} \mathrm{~d} y\right)\left(1+\epsilon_{u_{\mathcal{H}_{R A}}}\left(r^{*}\right)\right) \\
& +B_{u_{\mathcal{H}_{R}}} \frac{\left|V_{\sharp}\left(R_{1}\right)\right|^{\frac{1}{4}}}{\left|V_{\sharp}\left(r^{*}\right)\right|^{\frac{1}{4}}} \exp \left(i \int_{R_{1}}^{r^{*}}\left|V_{\sharp}(y)\right|^{\frac{1}{2}} \mathrm{~d} y\right)\left(1+\epsilon_{u_{\mathcal{H}_{R B}}}\left(r^{*}\right)\right),
\end{aligned}
$$

for

$$
A_{u_{\mathcal{H}_{R}}}=\frac{\mathfrak{W}\left(u_{\mathrm{WKB}_{B}}, u_{\mathcal{H}_{R}}\right)}{\mathfrak{W}\left(u_{\mathrm{WKB}_{B}}, u_{\mathrm{WKB}_{A}}\right)} \text { and } B_{u_{\mathcal{H}}}=\frac{\mathfrak{W}\left(u_{\mathrm{WKB}_{A}}, u_{\mathcal{H}_{R}}\right)}{\mathfrak{W}\left(u_{\mathrm{WKB}_{A}}, u_{\mathrm{WKB}_{B}}\right)} \text {. }
$$

Moreover,

$$
\begin{aligned}
& \sup _{r^{*} \in\left[R_{1}, R_{2}\right]}\left|\epsilon_{u_{\mathcal{H}_{R A}}}\left(r^{*}\right)\right| \lesssim 1, \\
& \sup _{r^{*} \in\left[R_{1}, R_{2}\right]}\left|\epsilon_{u_{\mathcal{H}_{R A}}}^{\prime}\left(r^{*}\right)\right| \lesssim \sup _{r^{*} \in\left[R_{1}, R_{2}\right]}\left|V_{\sharp}\right|^{\frac{1}{2}} \lesssim L^{\frac{1}{2}}+|m|+|\omega|, \\
& \epsilon_{u_{\mathcal{H}_{R A}}}\left(R_{1}\right)=\epsilon_{u_{\mathcal{H}_{R A}}}^{\prime}\left(R_{1}\right)=0,
\end{aligned}
$$

and analogously for $\epsilon_{u_{\mathcal{H}_{R B}}}$. Evaluating the Wronskians at $r^{*}=R_{1}$, we obtain

$$
\left|A_{u_{\mathcal{H}_{R}}}\right|,\left|B_{u_{\mathcal{H}_{R}}}\right| \lesssim 1
$$

in view of (8.51) and (8.52). This shows that

$$
\begin{aligned}
&\left\|u_{\mathcal{H}_{R}}\right\|_{L^{\infty}\left(-\infty, R_{2}\right)} \lesssim 1, \\
&\left\|u_{\mathcal{H}_{R}}{ }^{\prime}\right\|_{L^{\infty}\left(-\infty, R_{2}\right)} \lesssim|\omega|+|m|+L^{\frac{1}{2}} .
\end{aligned}
$$


To show the bound on $\partial_{\omega} u_{\mathcal{H}_{R}}$ we consider $u \tilde{\mathcal{H}}_{R}$. Then, $u \tilde{\mathcal{H}}_{R}$ satisfies the Volterra equation

$$
u_{\tilde{\mathcal{H}}_{R}}=1+\int_{-\infty}^{r^{*}} \frac{\tilde{K}\left(r^{*}, y\right)}{1+R_{1}-y}\left(1+R_{1}-y\right) \bar{V}(y) u_{\tilde{\mathcal{H}}_{R}}(y) \mathrm{d} y
$$

where $\tilde{K}\left(r^{*}, y\right)=e^{i\left(\omega-\omega_{+} m\right)\left(r^{*}-y\right)} \frac{\sin \left(\left(\omega-\omega_{+} m\right)\left(r^{*}-y\right)\right)}{\omega-\omega_{+} m}$. Completely analogous to before, it follows that

$$
\left\|u \tilde{\mathcal{H}}_{R}\right\|_{L^{\infty}\left(-\infty, R_{1}\right)} \lesssim 1 \text { and }\left\|u_{\tilde{\mathcal{H}}_{R}}^{\prime}\right\|_{L^{\infty}\left(-\infty, R_{1}\right)} \lesssim 1
$$

Now $\partial_{\omega} u \tilde{\mathcal{H}}_{R}$ solves

$$
\begin{aligned}
& \partial_{\omega} u \tilde{\mathcal{H}}_{R}=\int_{-\infty}^{r^{*}}\left(\partial_{\omega} \tilde{K}\left(r^{*}, y\right) \bar{V}(y)+\tilde{K}\left(r^{*}, y\right) \partial_{\omega} \bar{V}(y)\right) u_{\mathcal{H}_{R}}(y) \mathrm{d} y \\
& +\int_{-\infty}^{r^{*}} \frac{\tilde{K}\left(r^{*}, y\right)}{1+R_{1}-y}\left(1+R_{1}-y\right) \bar{V}(y) \partial_{\omega} u \tilde{\mathcal{H}}_{R}(y) \mathrm{d} y .
\end{aligned}
$$

As $\left|\partial_{\omega} \lambda_{m \ell}(a \omega)\right| \lesssim|m|$ from Lemma 3.2, we conclude that

$$
\left|\partial_{\omega} \bar{V}\right| \lesssim|\Delta| m \text { and }\left|\partial_{\omega} \tilde{K}\left(r^{*}, y\right)\right| \lesssim\left(r^{*}-y\right)^{2}
$$

such that

$$
\int_{-\infty}^{R_{1}}\left|\left(\partial_{\omega} \tilde{K}\left(r^{*}, y\right) \bar{V}(y)+\tilde{K}\left(r^{*}, y\right) \partial_{\omega} \bar{V}(y)\right) u_{\tilde{\mathcal{H}}_{R}}(y)\right| \mathrm{d} y \lesssim 1 .
$$

Again, by standard bounds on Volterra integral equations [94, Chapter $6, \S 10$ ] and using (8.60), (8.61), we obtain

$$
\left\|\partial_{\omega} u \tilde{\mathcal{H}}_{R}\right\|_{L^{\infty}\left(-\infty, R_{1}\right)} \lesssim 1
$$

and

$$
\left\|\partial_{\omega} u \tilde{\mathcal{H}}_{R}^{\prime}\right\|_{L^{\infty}\left(-\infty, R_{1}\right)} \lesssim 1
$$

This shows (8.45). Completely analogously we obtain (8.46) and (8.47). Now, we write

$$
\partial_{\omega} u_{\mathcal{H}_{R}}=\partial_{\omega}\left(e^{-i\left(\omega-\omega_{+} m\right) r^{*}} u_{\mathcal{H}_{R}}\right)=-i r^{*} u_{\mathcal{H}_{R}}+e^{-i\left(\omega-\omega_{+} m\right) r^{*}} \partial_{\omega} u_{\mathcal{H}_{R}}
$$


and

$$
\begin{aligned}
\partial_{\omega} u_{\mathcal{H}_{R}}{ }^{\prime}= & -i u_{\mathcal{H}_{R}}-i r^{*} u_{\mathcal{H}_{R}}{ }^{\prime}-i\left(\omega-\omega_{+} m\right) e^{-i\left(\omega-\omega_{+} m\right) r^{*}} \partial_{\omega} u \tilde{\mathcal{H}}_{R} \\
& +e^{-i\left(\omega-\omega_{+} m\right) r^{*}} \partial_{\omega} u_{\tilde{\mathcal{H}}_{R}}{ }^{\prime}
\end{aligned}
$$

Evaluating this at $r^{*}=R_{1}$ yields

$$
\left|\partial_{\omega} u_{\mathcal{H}_{R}}\right|\left(R_{1}\right) \lesssim\left|R_{1}\right| \lesssim \log (L)
$$

and

$$
\left|\partial_{\omega} u_{\mathcal{H}_{R}}{ }^{\prime}\right|\left(R_{1}\right) \lesssim \log (L)(|m|+|\omega|)
$$

This shows (8.42). The proofs of (8.43) and (8.44) are completely analogous.

Lemma 8.5 The renormalized transmission and reflection coefficients satisfy

$$
\begin{aligned}
& 2|\mathfrak{t}|=\left|\mathfrak{W}\left[u_{\mathcal{H}_{R}}, u_{\mathcal{C H}}\right]\right| \lesssim|m|+|\omega|+L^{\frac{1}{2}}, \\
& 2|\mathfrak{r}|=\left|\mathfrak{W}\left[u_{\mathcal{H}_{R}}, u_{\mathcal{C H}_{L}}\right]\right| \lesssim|m|+|\omega|+L^{\frac{1}{2}}
\end{aligned}
$$

for $m$ sufficiently large and all frequencies $\omega, \ell$. Moreover,

$$
\begin{aligned}
& \sup _{\omega \in\left(\omega_{-} m-1, \omega_{-} m+1\right)} 2\left|\partial_{\omega} \mathfrak{t}\right| \\
& =\sup _{\omega \in\left(\omega_{-} m-1, \omega_{-} m+1\right)}\left|\partial_{\omega} \mathfrak{W}\left[u_{\mathcal{H}_{R}}, u_{\mathcal{C} \mathcal{H}_{R}}\right]\right| \lesssim\left(|m|+L^{\frac{1}{2}}\right) \log (L) \\
& \sup _{\omega \in\left(\omega_{-} m-1, \omega_{-} m+1\right)} 2\left|\partial_{\omega} \mathfrak{r}\right| \\
& =\sup _{\omega \in\left(\omega_{-} m-1, \omega_{-} m+1\right)}\left|\partial_{\omega} \mathfrak{W}\left[u_{\mathcal{H}_{R}}, u_{\mathcal{C H}_{L}}\right]\right| \lesssim\left(|m|+L^{\frac{1}{2}}\right) \log (L) .
\end{aligned}
$$

and

$$
\begin{aligned}
& \left|\mathfrak{W}\left[\partial_{\omega} u_{\mathcal{H}_{R}}, u_{\mathcal{C H}_{R}}\right]\left(r^{*}\right)\right|+\left|\mathfrak{W}\left[\partial_{\omega} u_{\mathcal{H}_{R}}, u_{\mathcal{C H}_{L}}\right]\left(r^{*}\right)\right| \\
& \quad \lesssim \log (L)\left(|\omega|+|m|+L^{\frac{1}{2}}\right), \\
& \left|\mathfrak{W}\left[u_{\mathcal{H}_{R}}, \partial_{\omega} u_{\mathcal{C H}}\right]\left(r^{*}\right)\right|+\left|\mathfrak{W}\left[u_{\mathcal{H}_{R}}, \partial_{\omega} u_{\mathcal{C H}}\right]\left(r^{*}\right)\right| \\
& \quad \lesssim \log (L)\left(|\omega|+|m|+L^{\frac{1}{2}}\right)
\end{aligned}
$$

uniformly for $r^{*} \in\left[R_{1}, R_{2}\right]$ and $\left|\omega-\omega_{-} m\right| \leq 1, m$ sufficiently large. 
Proof The bounds (8.73) and (8.74) follow directly from Lemma 8.2 and Lemma 8.4. To show (8.75) we assume that $\left|\omega-\omega_{-} m\right| \leq 1$ and evaluate the Wronskian at $r^{*}=0$ :

$$
\begin{aligned}
\partial_{\omega} \mathfrak{W}\left[u_{\mathcal{H}_{R}}, u_{\mathcal{C H}_{R}}\right]= & \partial_{\omega} \mathfrak{W}\left[u_{\mathcal{H}_{R}}, u_{\mathcal{C H}_{R}}\right]\left(r^{*}=0\right) \\
= & \mathfrak{W}\left[\partial_{\omega} u_{\mathcal{H}_{R}}, u_{\mathcal{C H}_{R}}\right]\left(r^{*}=0\right) \\
& +\mathfrak{W}\left[u_{\mathcal{H}_{R}}, \partial_{\omega} u_{\mathcal{C H}}\right]\left(r^{*}=0\right) .
\end{aligned}
$$

Hence, (8.75) follows from (8.77). To show (8.77), we apply the fundamental theorem of calculus for $R_{1} \leq r^{*} \leq R_{2}$ and obtain

$$
\begin{aligned}
\left|\mathfrak{W}\left[\partial_{\omega} u_{\mathcal{H}_{R}}, u_{\mathcal{C H}}\right]\left(r^{*}\right)\right| \leq & \int_{R_{1}}^{r^{*}}\left|\partial_{r} * \mathfrak{W}\left[\partial_{\omega} u_{\mathcal{H}_{R}}, u_{\mathcal{C H}}\right]\right| \mathrm{d} r^{*} \\
& +\left|\mathfrak{W}\left[\partial_{\omega} u_{\mathcal{H}_{R}}, u_{\mathcal{C} \mathcal{H}_{R}}\right]\left(R_{1}\right)\right| .
\end{aligned}
$$

A direct computation shows

$$
\partial_{r^{*}} \mathfrak{W}\left[\partial_{\omega} u_{\mathcal{H}_{R}}, u_{\mathcal{C H}}\right]=-u_{\mathcal{H}_{R}} u_{\mathcal{C H}} \partial_{\boldsymbol{R}}\left(V_{\sharp}+V_{1}\right) .
$$

Thus, in view of Lemma 8.4 we obtain

$$
\sup _{r^{*} \in\left[R_{1}, R_{2}\right]}\left|\partial_{r^{*}} \mathfrak{W}\left[\partial_{\omega} u_{\mathcal{H}_{R}}, u_{\mathcal{C H}}\right]\right| \lesssim|m|+|\omega|
$$

From the proof of Lemma 8.4 we also have

$$
\left|\mathfrak{W}\left[\partial_{\omega} u_{\mathcal{H}_{R}}, u_{\mathcal{C H}}\right]\left(R_{1}\right)\right| \lesssim \log (L)\left(|\omega|+|m|+L^{\frac{1}{2}}\right)
$$

such that

$$
\sup _{r^{*} \in\left[R_{1}, R_{2}\right]}\left|\mathfrak{W}\left[\partial_{\omega} u_{\mathcal{H}_{R}}, u_{\mathcal{C H}_{R}}\right]\left(r^{*}\right)\right| \lesssim \log (L)\left(|\omega|+|m|+L^{\frac{1}{2}}\right)
$$

follows. Similarly, we obtain

$$
\sup _{r^{*} \in\left[R_{1}, R_{2}\right]}\left|\mathfrak{W}\left[u_{\mathcal{H}_{R}}, \partial_{\omega} u_{\mathcal{C H}}\right]\left(r^{*}\right)\right| \lesssim \log (L)(|\omega|+|m|)
$$

leading to (8.75) and (8.77). Completely analogously we obtain (8.76) as well as (8.78).

With the above lemma in hand we conclude 
Lemma 8.6 Let $m \in \mathbb{N}$ be sufficiently large and let $\epsilon>0$ be sufficiently small only depending on the black hole parameters. Then

$$
\begin{aligned}
\sup _{\left|\omega-\omega_{+} m\right| \leq \epsilon}\left\|\partial_{\omega} u \tilde{\mathcal{H}}_{R}\right\|_{L^{\infty}(-\infty, 0)} \lesssim L^{\frac{1}{2}} \log (L), \\
\sup _{\left|\omega-\omega_{-} m\right| \leq \epsilon}\left\|\partial_{\omega} u_{\mathcal{C} \tilde{\mathcal{H}}_{R}}\right\|_{L^{\infty}(0, \infty)} \lesssim L^{\frac{1}{2}} \log (L), \\
\sup _{\left|\omega-\omega_{-} m\right| \leq \epsilon}\left\|\partial_{\omega} u_{\mathcal{C} \mathcal{H}_{L}}\right\|_{L^{\infty}(0, \infty)} \lesssim L^{\frac{1}{2}} \log (L) .
\end{aligned}
$$

Proof We again only show the claim for $u \tilde{\mathcal{H}}_{R}$ as the other cases are completely analogous. Assume that $\left|\omega-\omega_{+} m\right| \leq \epsilon$ for some $\epsilon>0$ sufficiently small. In view of Lemma 8.4 it suffices to consider the region $r^{*} \in\left[R_{1}, 0\right]$. Now, note that

$$
\begin{aligned}
\partial_{\omega} u_{\mathcal{H}_{R}}= & \frac{1}{\mathfrak{W}\left[u_{\mathcal{C H}}, u_{\mathcal{C H}}\right]}\left(u_{\mathcal{C H}_{L}} \int_{R_{1}}^{r^{*}} u_{\mathcal{C H}_{R}} u_{\mathcal{H}_{R}} \partial_{\omega}\left(-V_{\sharp}-V_{1}\right)\right. \\
& \left.-u_{\mathcal{C H}} \int_{R_{1}}^{r^{*}} u_{\mathcal{H}_{R}} u_{\mathcal{C H}} \partial_{\omega}\left(-V_{\sharp}-V_{1}\right)\right) \\
& +\frac{\mathfrak{W}\left[\partial_{\omega} u_{\mathcal{H}_{R}}, u_{\mathcal{C H}}\right]\left(R_{1}\right)}{\mathfrak{W}\left[u_{\mathcal{C H}}, u_{\mathcal{C H}_{L}}\right]} u_{\mathcal{C H}}+\frac{\mathfrak{W}\left[\partial_{\omega} u_{\mathcal{H}_{R}}, u_{\mathcal{C H}}\right]\left(R_{1}\right)}{\mathfrak{W}\left[u_{\mathcal{C H}}, u_{\mathcal{C H}_{R}}\right]} u_{\mathcal{C H}_{L}} .
\end{aligned}
$$

Hence, using Lemma 8.4, Lemma 8.5,

$$
\sup _{r *\left[R_{1}, R_{2}\right]}\left|\partial_{\omega}\left(V_{\sharp}+V_{1}\right)\right| \lesssim|m|,
$$

as well as the lower bound $\left|\mathfrak{W}\left[u_{\mathcal{C H}_{R}}, u_{\mathcal{C H}_{L}}\right]\right| \gtrsim|m|$, we obtain

$$
\sup _{r^{*} \in\left[R_{1}, 0\right]}\left|\partial_{\omega} u_{\mathcal{H}_{R}}\right| \lesssim L^{\frac{1}{2}} \log (L) .
$$

In view of $u_{\mathcal{H}_{R}}=e^{i\left(\omega-\omega_{+} m\right) r^{*}} u_{\mathcal{H}_{R}}$ and the chain rule, the claim follows.

Lemma 8.7 The renormalized transmission and reflection coefficients satisfy

$$
\left|\mathfrak{t}^{\omega_{-}}\right| \gtrsim|m| \text { and }\left|\mathfrak{r}^{\omega_{-}}\right| \gtrsim|m|
$$

for all $m$ sufficiently large. 
Proof Throughout the proof we assume that $\omega=\omega_{-} m$. As $u_{\mathcal{C H}_{R}}=u_{\mathcal{C H}_{L}}$ for $\omega=\omega_{-} m$, it suffices to bound the Wronskian $\left|\mathfrak{W}\left[u_{\mathcal{H}_{R}}, u_{\mathcal{C H}}\right]\right|$ from below. To do so, let $\mathfrak{A}$ and $\mathfrak{B}$ be the unique coefficients satisfying

$$
u_{\mathcal{C} \mathcal{H}_{R}}=\mathfrak{A} u_{\mathcal{H}_{R}}+\mathfrak{B} u_{\mathcal{H}_{L}}
$$

From $u_{\mathcal{C H}_{R}}=\overline{u_{\mathcal{C H}}}$ it follows that

$$
u_{\mathcal{C H}}=2 \operatorname{Re}\left(\mathfrak{A} u_{\mathcal{H}_{R}}\right) .
$$

Now, for $\epsilon>0$ to be chosen later, define

$$
R_{2}^{\epsilon}:=\frac{1}{2\left|\kappa_{-}\right|} \log (L)+\frac{1}{\epsilon} .
$$

Now, $u_{\mathcal{C H}}-1$ is a solution to the Volterra equation

$$
u_{\mathcal{C} \mathcal{H}_{R}}-1=\int_{r^{*}}^{\infty} \frac{y-r^{*}}{y-R_{2}^{\epsilon}}\left(y-R_{2}^{\epsilon}\right) \tilde{V}(y)\left[\left(u_{\mathcal{C H}}-1\right)+1\right] \mathrm{d} y,
$$

where $\tilde{V}=V_{1}+V_{\sharp}\left(\omega=\omega_{-} m\right)$. We have

$$
\int_{R_{2}^{\epsilon}}^{\infty}\left(y-R_{2}^{\epsilon}\right) \tilde{V}(y) \lesssim L e^{-2\left|\kappa_{-}\right| R_{2}^{\epsilon}} \lesssim e^{-\frac{2\left|\kappa_{-}\right|}{\epsilon}} .
$$

Using bounds on solutions to Volterra integral equations as before (see [94, Chapter 6, §10]), we obtain that

$$
\left\|u_{\mathcal{C H}}-1\right\|_{L^{\infty}\left(R_{2}^{\epsilon}, \infty\right)}<\frac{1}{2}
$$

for $\epsilon>0$ sufficiently small enough. Thus,

$$
\frac{1}{2}<u_{\mathcal{C H}}\left(R_{2}^{\epsilon}\right)=2 \operatorname{Re}\left(\mathfrak{A} u_{\mathcal{H}_{R}}\left(R_{2}^{\epsilon}\right)\right) \lesssim 2|\mathfrak{A}|\left\|u_{\mathcal{H}_{R}}\right\|_{L^{\infty}\left(-\infty, R_{2}^{\epsilon}\right)}
$$

Note that (8.39) also holds if we replace $R_{2}$ by $R_{2}^{\epsilon}$ for some fixed value of $\epsilon>0$. Thus, we conclude that $|\mathfrak{B}|=|\mathfrak{A}| \gtrsim 1$ which shows

$$
\left|\mathfrak{W}\left[u_{\mathcal{H}_{R}}, u_{\mathcal{C H}}\right]\right| \gtrsim\left(\omega_{-}-\omega_{+}\right)|m| \gtrsim|m| .
$$

This concludes the proof. 


\subsection{Scattering poles: representation formula for $\psi$ on the interior}

Proposition 8.1 Let $\psi_{0} \in C_{c}^{\infty}\left(\mathcal{H}_{R}\right)$ and assume that $\psi_{0}$ is only supported on azimuthal modes $m \geq m_{0}$ for some $m_{0}$ sufficiently large. Let $\tilde{\psi} \in C^{\infty}(\mathcal{B})$ be the arising solution of (1.2) with vanishing data on $\mathcal{H}_{L} \cup \mathcal{B}_{\mathcal{H}}$ and $\psi\left\lceil_{\mathcal{H}_{R}}=\psi_{0}\right.$. Then,

$$
\begin{aligned}
\tilde{\psi}\left(v, r, \theta, \tilde{\phi}_{+}\right)= & \frac{1}{\sqrt{2 \pi\left(r^{2}+a^{2}\right)}} \\
& \sum_{m \ell} \int_{\mathbb{R}} e^{-i \omega\left(v-r^{*}\right)} e^{i m\left(\tilde{\phi}_{+}-\omega_{+} r^{*}\right)} S_{m \ell}(a \omega, \cos \theta) \mathcal{F}_{\mathcal{H}}\left[\psi_{0}\right] u_{\mathcal{H}_{R}} \mathrm{~d} \omega,
\end{aligned}
$$

where $u_{\mathcal{H}_{R}}$ is defined in (8.1) and

$$
\begin{aligned}
& \mathcal{F}_{\mathcal{H}}\left[\psi_{0}\right](\omega, m, \ell) \\
& \quad:=\frac{\sqrt{r_{+}^{2}+a^{2}}}{\sqrt{2 \pi}} \int_{\mathbb{S}^{2}} \int_{\mathbb{R}} \psi_{0}\left(v, \theta, \tilde{\phi}_{+}\right) e^{i \omega v} e^{-i m \tilde{\phi}_{+}} S_{m \ell}(a \omega, \cos \theta) \mathrm{d} \sigma_{\mathbb{S}^{2}} \mathrm{~d} v .
\end{aligned}
$$

Moreover, in $\mathcal{B}$ we have

$$
\begin{aligned}
\tilde{\psi}\left(v, r, \theta, \tilde{\phi}_{-}\right)= & \frac{1}{\sqrt{2 \pi\left(r^{2}+a^{2}\right)}} \sum_{m \ell} \text { p.v. } \int_{\mathbb{R}} e^{-i \omega\left(v-r^{*}\right)} e^{i m\left(\tilde{\phi}_{-}-\omega_{-} r^{*}\right)} \\
& \times S_{m \ell}(a \omega, \cos \theta) \mathcal{F}_{\mathcal{H}}\left[\psi_{0}\right] \frac{\mathfrak{t}(\omega, m, \ell)}{\omega-\omega_{-} m} u_{\mathcal{C H}} \mathrm{d} \omega \\
& +\frac{1}{\sqrt{2 \pi\left(r^{2}+a^{2}\right)}} \sum_{m \ell} \text { p.v. } \int_{\mathbb{R}} e^{-i \omega\left(v-r^{*}\right)} e^{i m\left(\tilde{\phi}_{-}-\omega_{-} r^{*}\right)} \\
& \times S_{m \ell}(a \omega, \cos \theta) \mathcal{F}_{\mathcal{H}}\left[\psi_{0}\right] \frac{\mathfrak{r}(\omega, m, \ell)}{\omega-\omega_{-} m} u_{\mathcal{C H}_{R}} \mathrm{~d} \omega
\end{aligned}
$$

as well as

$$
\begin{array}{r}
\tilde{\psi}\left(u, r, \theta, \phi_{-}^{*}\right)=\frac{1}{\sqrt{2 \pi\left(r^{2}+a^{2}\right)}} \sum_{m \ell} \text { p.v. } \int_{\mathbb{R}} e^{i \omega\left(u-r^{*}\right)} e^{i m\left(\phi_{-}^{*}+\omega_{-} r^{*}\right)} \\
S_{m \ell}(a \omega, \cos \theta) \mathcal{F}_{\mathcal{H}}\left[\psi_{0}\right] \frac{\mathfrak{t}(\omega, m, \ell)}{\omega-\omega_{-} m} u_{\mathcal{C H}} \mathrm{d} \omega
\end{array}
$$




$$
\begin{gathered}
+\frac{1}{\sqrt{2 \pi\left(r^{2}+a^{2}\right)}} \sum_{m \ell} \text { p.v. } \int_{\mathbb{R}} e^{i \omega\left(u-r^{*}\right)} e^{i m\left(\phi_{-}^{*}+\omega_{-} r^{*}\right)} \\
S_{m \ell}(a \omega, \cos \theta) \mathcal{F}_{\mathcal{H}}\left[\psi_{0}\right] \frac{\mathfrak{r}(\omega, m, \ell)}{\omega-\omega_{-} m} u_{\mathcal{C H}} \mathrm{d} \omega
\end{gathered}
$$

for $r \leq \frac{r_{+}+r_{-}}{2}$

Proof Note that $\mathcal{F}_{\mathcal{H}}\left[\psi_{0}\right](\omega, m, \ell)$ is rapidly decaying in $\omega, m, \ell$ and smooth in $\omega$ which follows from the fact that $\psi_{0} \in C_{c}^{\infty}\left(\mathcal{H}_{R}\right)$. Moreover, using Lemma 8.2 and Lemma 8.4, we have that the right hand side of (8.101) is a smooth solution to (1.2) in the interior region $\mathcal{B}$. Now, note that the right hand side of $(8.101)$ converges to $\psi_{0}$ as $r \rightarrow r_{+}$for fixed $v$. Similarly, after a change of coordinates to $\left(u, r, \theta, \tilde{\phi}_{+}\right)$we obtain $\tilde{\psi}$ converges to zero as $r \rightarrow r_{+}$and $u$ fixed in view of the Riemann-Lebesgue lemma. Thus, (8.101) follows from the uniqueness of the characteristic problem.

In order to show (8.103) we first write the right-hand side of (8.101) as a principal value integral and then use the definition of the reflection and transmission coefficients from Definition 8.2 to replace $u_{\mathcal{H}_{R}}$ with

$$
u_{\mathcal{H}_{R}}=\frac{\mathfrak{t}(\omega, m, \ell)}{\omega-\omega_{-} m} u_{\mathcal{C H}_{L}}+\frac{\mathfrak{r}(\omega, m, \ell)}{\omega-\omega_{-} m} u_{\mathcal{C H}_{R}}
$$

In order to use linearity of the principal value to write $\tilde{\psi}$ as a sum of two terms as in (8.103), it suffices to show that

$$
\sum_{m \ell} \text { p.v. } \int_{\mathbb{R}} e^{-i \omega\left(v-r^{*}\right)} e^{i m\left(\tilde{\phi}_{+}-\omega_{+} r^{*}\right)} S_{m \ell}(a \omega, \cos \theta) \mathcal{F}_{\mathcal{H}}\left[\psi_{0}\right] \frac{\mathfrak{t}(\omega, m, \ell)}{\omega-\omega \_m} u_{\mathcal{C H}} \mathrm{d} \omega
$$

converges locally uniformly. Note that the other term with $\mathfrak{t}(\omega, m, \ell) u_{\mathcal{C}} \mathcal{H}_{L}$ replaced by $\mathfrak{r}(\omega, m, \ell) u_{\mathcal{C H}_{R}}$ is treated completely analogously.

In the following we will be brief because in the proof of Theorem 1, where we have to quantitatively control terms of the form (8.106), we will indeed show stronger estimates and provide more details. First, in view of the facts that $\mathcal{F}_{\mathcal{H}}\left[\psi_{0}\right](\omega, m, \ell)$ is rapidly decaying in $\omega, m, \ell$, that $\left\|S_{m \ell}(a \omega, \cos \theta)\right\|_{L^{2}((-\pi, \pi) ; \cos \theta \mathrm{d} \theta)}=1$, and that we have uniform (polynomial) bounds on $|\mathfrak{t}(\omega, m, \ell)|$ and $\left\|u_{\mathcal{C H}}\right\|_{L} \|_{\left(\left[R_{1},+\infty\right)\right)}$ (see Lemma 8.2, Lemma 8.4, Lemma 8.5), it suffices to consider frequencies in the range $\left|a \omega-a \omega_{-} m\right|<\frac{1}{m}$. Now, uniformly in $\left|a \omega-a \omega_{-} m\right|<\frac{1}{m}$, we have polynomial bounds in $\omega, m, \ell$ on $\left\|\partial_{\omega} S_{m \ell}(a \omega, \cos \theta)\right\|_{L^{2}((-\pi, \pi) ; \cos \theta \mathrm{d} \theta)}$, $\left\|\partial_{\omega} u_{\mathcal{C} \mathcal{H}_{L}}\right\|_{L^{\infty}(0,+\infty)}$ and $\left|\partial_{\omega} \mathfrak{t}(\omega, m, \ell)\right|$ as shown in Proposition 3.3, Lemma 8.6 and Lemma 8.5, respectively. Moreover, again using the bound on 
$\left\|\partial_{\omega} S_{m \ell}(a \omega, \cos \theta)\right\|_{L^{2}}$ and the fact that $\psi_{0}$ is compactly supported, we also obtain that $\sup _{\left|a \omega-a \omega_{-} m\right|<\frac{1}{m}}\left|\partial_{\omega} \mathcal{F}_{\mathcal{H}}\left[\psi_{0}\right]\right|$ is rapidly decaying in $m, \ell$. This shows that (8.106) converges locally uniformly for $r^{*} \geq 0, v \in \mathbb{R}$ with values in $L^{2}\left(\mathbb{S}^{2}\right)$. This shows that, after a change of coordinates, (8.103) and (8.104) hold true pointwise for $r \leq \frac{r_{-}+r_{+}}{2}, v \in \mathbb{R}$ and in $L^{2}\left(\mathbb{S}^{2}\right)$. Finally, using standard elliptic estimates and the fundamental theorem of calculus, we also have polynomial bounds in $\omega, m, \ell$ on $\left\|S_{m \ell}(a \omega, \cos \theta)\right\|_{L^{\infty}(-\pi, \pi)}$, as well as polynomial bounds in $m, \ell$ on $\sup _{\left|a \omega-a \omega_{-} m\right|<\frac{1}{m}}\left\|\partial_{\omega} S_{m \ell}(a \omega, \cos \theta)\right\|_{L^{\infty}(-\pi, \pi)}$. Thus, both terms on the right-hand side of (8.103) are continuous and the equality (8.103) holds pointwise. We obtain the analogous result for (8.104).

Before we prove the blow-up result in Sect. 9, we need one more final ingredient which is a consequence of the domain of dependence.

Lemma 8.8 Let $\tilde{\psi} \in C^{\infty}(\mathcal{B})$ be a solution to (1.2) arising from vanishing data on $\mathcal{H}_{L} \cup \mathcal{B}_{\mathcal{H}}$ and compatible smooth data $\psi_{0} \in C^{\infty}\left(\mathcal{H}_{R}\right)$. Then, $\tilde{\psi}\left(u_{0}, r_{0}, \theta_{0}, \phi_{-}^{*}\right)$ onlydepends on $\psi_{0} \uparrow_{\left\{v \leq 2 r^{*}\left(r_{0}\right)-u_{0}+\tilde{c}\right\}}$, where $\tilde{c}=\tilde{c}(\mathfrak{p}, l)>0$ is a constant.

Proof In coordinates $\left(v, r, \theta, \tilde{\phi}_{-}\right)$(or equivalently in coordinates $\left(v, r, \theta, \tilde{\phi}_{+}\right)$) define the function $\tilde{v}:=v+f(r)$ on $\mathcal{B}$ and choose $f$ to satisfy

$$
\frac{\mathrm{d} f}{\mathrm{~d} r}=-\sqrt{\frac{a^{2}}{\Xi} \frac{1}{|\Delta|}}
$$

with initial condition $f\left(r_{+}\right)=0$. This is well defined as $\frac{1}{\sqrt{|\Delta|}}$ is integrable at the event and Cauchy horizons. Now $f$ is non-negative and satisfies $\sup _{r \in\left(r_{-}, r_{+}\right)} f \leq \tilde{c}$ for a constant $\tilde{c}>0$ only depending on the black hole parameters. A computation also shows that, uniformly on $\mathcal{B}$, we have

$$
g_{\text {KAdS }}(\nabla \tilde{v}, \nabla \tilde{v})=\frac{a^{2} \sin ^{2} \theta}{\Sigma \Delta_{\theta}}-\frac{a^{2}}{\Sigma \Xi}<0 \text { and } g_{\text {KAdS }}(\nabla \tilde{v},-\nabla r)<0 .
$$

This means that $\nabla \tilde{v}$ is a future-directed timelike vector field. Thus, the level sets of the function $\tilde{v}$ are spacelike.

Now, consider

$$
\tilde{\psi}\left(u_{0}, r_{0}, \theta_{0}, \phi_{-}^{*}\right) .
$$

Since $\nabla \tilde{v}$ is future directed and timelike, it follows from the domain of dependence that (8.109) only depends on 


$$
\psi_{0}\left\lceil_{\left\{\tilde{v}\left(v, r_{+}\right) \leq \tilde{v}\left(v\left(r_{0}, u_{0}\right), r_{0}\right)\right\}}=\psi_{0}\left\lceil\left\{v \leq 2 r^{*}\left(r_{0}\right)-u_{0}+f\left(r_{0}\right)\right\},\right.\right.
$$

since $\tilde{v}\left(v\left(r_{0}, u_{0}\right), r_{0}\right)=2 r^{*}\left(r_{0}\right)-u_{0}+f\left(r_{0}\right)$. This concludes the proof.

We will now finally turn to the proof of Theorem 1 .

\section{Proof of Theorem 1: Small divisors lead to blow-up}

We recall that the cosmological constant $\Lambda<0$ (and thus $l=\sqrt{-3 / \Lambda}>0$ ) was arbitrary but fixed as in (2.6).

Theorem 1 Conjecture 5 holds true.

More precisely, let the dimensionless black hole parameters $(\mathfrak{m}, \mathfrak{a}) \in$

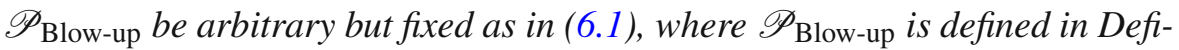
nition 5.3.

Let $\psi \in C^{\infty}\left(\mathcal{M}_{\mathrm{KAdS}} \backslash \mathcal{C H}\right)$ be the unique solution to (1.2) arising from the smooth and compactly supported initial data specified in Definition 6.2 on Kerr-AdS with parameters $(M, a)=(\mathfrak{m} / \sqrt{-\Lambda}, \mathfrak{a} / \sqrt{-\Lambda})$.

Then, for each $u_{0} \in \mathbb{R}$, the solution $\psi$ blows up at the Cauchy horizon $\mathrm{CH}_{R}$ as

$$
\lim _{r \rightarrow r_{-}}\left\|\psi\left(u_{0}, r\right)\right\|_{L^{2}\left(\mathbb{S}^{2}\right)}^{2}=+\infty
$$

Moreover, $\mathscr{P}_{\text {Blow-up }} \subset \mathscr{P}$ has the following properties:

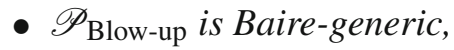

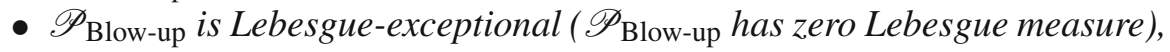

- $\mathscr{P}_{\text {Blow-up }}$ has full packing dimension $\operatorname{dim}_{P}\left(\mathscr{P}_{\text {Blow-up }}\right)=2$.

From our proof we will also obtain the following corollary which gives a genericity condition for compactly supported initial data which lead to blowup as in (9.1).

Corollary 1 Let the dimensionless black hole parameters $(\mathfrak{m}, \mathfrak{a}) \in \mathscr{P}_{\text {Blow-up }}$ be arbitrary but fixed as in (6.1). Let $\tilde{\Psi}_{0}, \tilde{\Psi}_{1} \in C_{c}^{\infty}\left(\Sigma_{0}\right)$ be arbitrary initial data satisfying the following genericity condition

$$
\sum_{i \in \mathbb{N}}\left|m_{i} e^{\sqrt{\left|m_{i}\right|}}\right|^{2}\left|G\left(\tilde{\Psi}_{0}, \tilde{\Psi}_{1}, m_{i}, \ell_{i}\right)\right|^{2}=+\infty
$$

where $m_{i}, \ell_{i}$ are the subsequences in (6.2) associated to the non-Diophantine condition (i.e. the choice of $\mathfrak{p} \in \mathscr{P}_{\text {Blow-up }}$ ) and 


$$
\begin{aligned}
& G\left(\tilde{\Psi}_{0}, \tilde{\Psi}_{1}, m, \ell\right) \\
& :=\int_{r_{+}}^{\infty} \int_{\mathbb{S}^{2}}\left\{\frac{\Sigma}{\sqrt{r^{2}+a^{2}}} u_{\infty}\left(\omega=\omega_{-} m, m, \ell\right) e^{-i m \phi} S_{m \ell}\left(a \omega_{-} m, \cos \theta\right)\right. \\
& \left.\quad \times\left(-2 \sqrt{-g^{t t}} \tilde{\Psi}_{1}(r, \theta, \phi)-i \omega_{-} m g^{t t} \tilde{\Psi}_{0}(r, \theta, \phi)\right)\right\} \mathrm{d} \sigma_{\mathbb{S}^{2}} \mathrm{~d} r .
\end{aligned}
$$

Then, the arising solution $\tilde{\psi} \in C^{\infty}\left(\mathcal{M}_{\mathrm{KAdS}} \backslash \mathcal{C H}\right)$ to $(1.2)$ with $\left(\tilde{\psi}\left\lceil\Sigma_{0}\right.\right.$ , $\left.n_{\Sigma_{0}} \tilde{\psi} \uparrow_{\Sigma_{0}}\right)=\left(\tilde{\Psi}_{0}, \tilde{\Psi}_{1}\right)$, vanishing incoming data on $\mathcal{H}_{L} \cup \mathcal{B}_{\mathcal{H}}$ and with Dirichlet boundary conditions imposed at infinity, blows up at the Cauchy horizon $\mathcal{C H}_{R}$ for every $u_{0} \in \mathbb{R}$ as

$$
\lim _{r \rightarrow r_{-}}\left\|\tilde{\psi}\left(u_{0}, r\right)\right\|_{L^{2}\left(\mathbb{S}^{2}\right)}^{2}=+\infty .
$$

Corollary 1 will be an immediate consequence of the proof of Theorem 1 and will be given thereafter. Also note that the initial data which we construct in Sect. 6 do indeed satisfy the genericity condition of (9.2) as shown in Lemma 6.2.

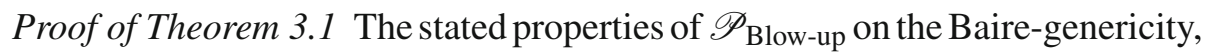
the zero Lebesgue measure and the full packing dimension follow from Proposition 5.2, Proposition 5.3 and Proposition 5.4, respectively.

We now turn to the proof of (9.1). First, we write $\psi_{0}:=\psi\left\lceil\mathcal{H}_{R}\right.$ and note that

$$
D:=\sum_{0 \leq i+j \leq 4} \int_{\mathbb{R} \times \mathbb{S}^{2}}\left|\nabla^{i} K_{+}^{j} \psi_{0}\left(v, \theta, \tilde{\phi}_{+}\right)\right|^{2} \mathrm{~d} \sigma_{\mathbb{S}^{2}} \mathrm{~d} v<\infty
$$

in view of Theorem 2 and Proposition 2.1. Now, let $u_{0} \in \mathbb{R}$ be fixed and let $r_{n}^{*} \rightarrow \infty$ be a sequence with $r_{n}^{*}>r_{0}^{*}$ for sufficiently large $r_{0}^{*}$. We will first prove

Proposition 9.1 For all $r_{n}^{*} \geq r_{0}$, we have that

$$
\begin{aligned}
\left\|\psi\left(u_{0}, r_{n}^{*}\right)\right\|_{L^{2}\left(\mathbb{S}^{2}\right)}^{2} & =\sum_{m \ell}\left|\pi \frac{\mathfrak{r}^{\omega_{-}}(m, \ell){\tilde{\mathcal{C}_{\mathcal{H}}}}^{\omega_{-}}\left(r_{n}^{*}, m, \ell\right)}{\sqrt{r_{n}^{2}+a^{2}}} a_{\mathcal{H}}^{R_{n}}\left(\omega=\omega_{-} m, m, \ell\right)\right|^{2} \\
& +\operatorname{Err}(D),
\end{aligned}
$$

where $|\operatorname{Err}(D)| \lesssim u_{0} D$ uniformly for all $r_{n}^{*} \geq r_{0}^{*}$ and $R_{n}:=2 r_{n}^{*}-u_{0}+$ $\tilde{c}$. Also recall the definition of $a_{\mathcal{H}}^{R}$ in (7.34). Here we also use the notation $u_{\tilde{\mathcal{C H}}_{L}}{ }^{\omega-}\left(r_{n}^{*}, m, \ell\right):=u_{\tilde{\mathcal{H}}_{L}}\left(r_{n}^{*}, \omega=\omega_{-} m, m, \ell\right)$.

Once we have established (9.6), the blow-up result of (9.1) will be proved. We now turn to the proof of Proposition 9.1. 
Proof of Proposition 9.1 In view of the domain of dependence property as stated in Lemma 8.8, we have that $\psi\left(u_{0}, r_{n}^{*}, \theta, \phi_{-}^{*}\right)$ only depends on $\psi_{0} \Upsilon_{\left\{v \leq 2 r_{n}^{*}-u_{0}+\tilde{c}\right\}}$. Consider now

$$
\psi_{0}^{n}\left(v, \theta, \tilde{\phi}_{+}\right):=\psi_{0}^{R_{n}}\left(v, \theta, \tilde{\phi}_{+}\right)
$$

where $\psi_{0}^{R_{n}}\left(v, \theta, \tilde{\phi}_{+}\right)=\psi_{0}\left(v, \theta, \tilde{\phi}_{+}\right) \chi\left(R_{n}-v\right)$ is defined in (7.3) with $R_{n}=$ $2 r_{n}^{*}-u_{0}+\tilde{c}$. Now, $\psi\left(u_{0}, r_{n}^{*}, \theta, \phi_{-}^{*}\right)$ only depends on $\psi_{0}^{n}$.

Using the representation formula (8.104) in Proposition 8.1 we write

$$
\begin{aligned}
\psi\left(u_{0}, r_{n}^{*}, \theta, \phi_{-}^{*}\right)= & \frac{1}{\sqrt{2 \pi\left(r_{n}^{2}+a^{2}\right)}} \sum_{m \ell} \text { p.v. } \int_{\mathbb{R}} e^{i \omega\left(u_{0}-r_{n}^{*}\right)} e^{i m\left(\phi_{-}^{*}+\omega_{-} r_{n}^{*}\right)} \\
& \times S_{m \ell}(a \omega, \cos \theta) \mathcal{F}_{\mathcal{H}}\left[\psi_{0}^{n}\right] \frac{\mathfrak{t}(\omega, m, \ell)}{\omega-\omega_{-} m} u_{\mathcal{C H}} \mathrm{d} \omega \\
& +\frac{1}{\sqrt{2 \pi\left(r_{n}^{2}+a^{2}\right)}} \sum_{m \ell} \text { p.v. } \int_{\mathbb{R}} e^{i \omega\left(u_{0}-r_{n}^{*}\right)} e^{i m\left(\phi_{-}^{*}+\omega_{-} r_{n}^{*}\right)} \\
& \times S_{m \ell}(a \omega, \cos \theta) \mathcal{F}_{\mathcal{H}}\left[\psi_{0}^{n}\right] \frac{\mathfrak{r}(\omega, m, \ell)}{\omega-\omega_{-} m} u_{\mathcal{C H}} \mathrm{d} \omega \\
= & : I+I I .
\end{aligned}
$$

We consider both terms individually and start with the term $I$. Moreover, we split the term $I$ into $\left|a \omega-a \omega_{-} m\right|<\frac{1}{m}$ and $\left|a \omega-a \omega_{-} m\right| \geq \frac{1}{m}$ and call the terms $I_{\text {res }}$ and $I_{\text {non-res, }}$, respectively, such that $I=I_{\text {res }}+I_{\text {non-res. }}$. First, we claim that the spherical $L^{2}$-norm of the term

$$
\begin{aligned}
I_{\text {non-res }}= & \frac{1}{\sqrt{2 \pi\left(r_{n}^{2}+a^{2}\right)}} \sum_{m \ell} \text { p.v. } \int_{\left|\omega_{-} m-\omega\right| \geq \frac{1}{a m}} e^{i \omega\left(u_{0}-r_{n}^{*}\right)} e^{i m\left(\phi_{-}^{*}+\omega_{-} r_{n}^{*}\right)} \\
& \times S_{m \ell}(a \omega, \cos \theta) \mathcal{F}_{\mathcal{H}}\left[\psi_{0}^{n}\right] \frac{\mathfrak{t}(\omega, m, \ell)}{\omega-\omega_{-} m} u_{\mathcal{C H}} \mathrm{d} \omega
\end{aligned}
$$

is controlled by $D$ uniformly as $r_{n}^{*} \rightarrow \infty$.

Lemma 9.1 We have $\left\|I_{\text {non-res }}\right\|_{L^{2}\left(\mathbb{S}^{2}\right)}^{2}\left(r_{n}^{*}, u_{0}\right) \lesssim D$ for all $r_{n}^{*} \geq r_{0}^{*}$.

Proof Using $\left|\frac{1}{\omega-\omega_{-} m}\right| \leq a m$ in the integrand of (9.9) and $\int_{0}^{2 \pi} e^{i(m-\tilde{m}) \phi} \mathrm{d} \phi=$ $2 \pi \delta_{m \tilde{m}}$ we estimate 
$\left\|I_{\text {non-res }}\right\|_{L^{2}\left(\mathbb{S}^{2}\right)}^{2} \lesssim \sum_{m} m^{2} \int_{0}^{\pi}\left|\sum_{\ell \geq|m|} \int_{\mathbb{R}}\right| S_{m \ell}(a \omega, \cos \theta) \mathcal{F}_{\mathcal{H}}\left[\psi_{0}^{n}\right] \mathfrak{t} u_{\mathcal{C H}}|\mathrm{d} \omega|^{2}$ $\sin \theta \mathrm{d} \theta$.

From the Cauchy-Schwarz inequality as well as Lemma 8.2, Lemma 8.4 and Lemma 8.5, we obtain

$$
\begin{aligned}
\left\|I_{\text {non-res }}\right\|_{L^{2}\left(\mathbb{S}^{2}\right)}^{2} \lesssim & \sum_{m}\left[\int_{0}^{\pi} \sum_{\tilde{\ell} \geq|m|} \int_{\mathbb{R}} \frac{\left|S_{m \tilde{\ell}}(a \omega, \cos \theta)\right|^{2}}{\left(1+\omega^{2}\right)\left(1+\Lambda_{m \tilde{\ell}}\right)} \mathrm{d} \omega \sin \theta \mathrm{d} \theta\right. \\
& \left.\times \sum_{\ell \geq|m|} \int_{\mathbb{R}}\left(1+\omega^{2}\right)\left(1+\Lambda_{m \ell}\right) m^{2}\left(1+\omega^{2}+\Lambda_{m \ell}\right)\left|\mathcal{F}_{\mathcal{H}}\left[\psi_{0}^{n}\right]\right|^{2} \mathrm{~d} \omega\right] \\
\lesssim & \sum_{m} \sum_{\ell \geq|m|} \int_{\mathbb{R}}\left(1+\omega^{2}\right)\left(1+\Lambda_{m \ell}\right) m\left(1+\omega^{2}+\Lambda_{m \ell}\right)\left|\mathcal{F}_{\mathcal{H}}\left[\psi_{0}^{n}\right]\right|^{2} \mathrm{~d} \omega \lesssim D,
\end{aligned}
$$

where we have used that $\Lambda_{m \ell} \geq \Xi^{2} \ell(\ell+1)$ such that $\sum_{\tilde{\ell} \geq|m|} \frac{1}{1+\Lambda_{m \tilde{\ell}}} \lesssim \frac{1}{m}$

Now, we turn to the term $I_{\text {res }}$ :

$$
\begin{aligned}
I_{\text {res }}= & \frac{1}{\sqrt{2 \pi\left(r_{n}^{2}+a^{2}\right)}} \sum_{m \ell} \text { p.v. } \int_{\omega_{-} m-\frac{1}{a m}}^{\omega-m+\frac{1}{a m}} e^{i \omega\left(u_{0}-r_{n}^{*}\right)} e^{i m\left(\phi_{-}^{*}+\omega_{-} r_{n}^{*}\right)} \\
& \times S_{m \ell}(a \omega, \cos \theta) \mathcal{F}_{\mathcal{H}}\left[\psi_{0}^{n}\right] \frac{\mathfrak{t}(\omega, m, \ell)}{\omega-\omega_{-} m} u_{\mathcal{C} \mathcal{H}_{L}} \mathrm{~d} \omega
\end{aligned}
$$

and write $u_{\mathcal{C H}_{L}}=e^{-i\left(\omega-\omega_{-} m\right) r^{*}} u_{\tilde{\mathcal{C H}}_{L}}$. Then,

$$
\begin{aligned}
& I_{\text {res }}=I_{\text {res }}^{a}+I_{\text {res }}^{b}=\frac{1}{\sqrt{2 \pi\left(r_{n}^{2}+a^{2}\right)}} \sum_{m \ell} \text { p.v. } \int_{\omega_{-} m-\frac{1}{a m}}^{\omega-m+\frac{1}{a m}} \frac{e^{-2 i\left(\omega-\omega_{-} m\right) r_{n}^{*}} e^{i \omega u_{0}} \mathcal{F}_{\mathcal{H}}\left[\psi_{0}^{n}\right]}{\omega-\omega_{-} m} \mathrm{~d} \omega \\
& \times e^{i m \phi_{-}^{*}} S_{m \ell}\left(a \omega_{-} m, \cos \theta\right) \mathfrak{t}\left(\omega_{-} m, m, \ell\right){\tilde{\mathcal{C H}_{L}}}^{\omega_{-}} \\
& +\frac{1}{\sqrt{2 \pi\left(r_{n}^{2}+a^{2}\right)}} \sum_{m \ell} \int_{\omega_{-} m-\frac{1}{a m}}^{\omega_{-} m+\frac{1}{a m}} e^{-2 i\left(\omega-\omega_{-} m\right) r_{n}^{*}} e^{i \omega u_{0}} \mathcal{F}_{\mathcal{H}}\left[\psi_{0}^{n}\right] e^{i m \phi_{-}^{*}} \\
& \times\left[S_{m \ell}\left(a \omega \_m, \cos \theta\right) \partial_{\omega}\left(\mathfrak{t}(\omega, m, \ell) \tilde{u}_{\mathcal{C}} \mathcal{H}_{L}\right)(\tilde{\xi})\right. \\
& \left.+\mathfrak{t}(\omega, m, \ell) \tilde{\mathcal{C}}_{\mathcal{H}_{L}} \frac{S_{m \ell}(a \omega, \cos \theta)-S_{m \ell}\left(a \omega \_m, \cos \theta\right)}{\omega-\omega_{-} m}\right] \mathrm{d} \omega
\end{aligned}
$$


for some $\tilde{\xi}=\tilde{\xi}(\omega) \in\left(\omega_{-} m-\frac{1}{a m}, \omega_{-} m+\frac{1}{a m}\right)$ in view of the mean value theorem. Again, we consider both terms $I_{\text {res }}^{a}$ and $I_{\text {res }}^{b}$ individually and begin with term $I_{\text {res }}^{b}$.

Lemma 9.2 We have $\left\|I_{\text {res }}^{b}\right\|_{L^{2}\left(\mathbb{S}^{2}\right)}^{2}\left(r_{n}^{*}, u_{0}\right) \lesssim D$ for all $r_{n}^{*} \geq r_{0}^{*}$.

Proof We decompose the term $I_{\text {res }}^{b}=I_{\text {res }}^{b 1}+I_{\text {res }}^{b 2}$ further into the two summands appearing in the $\omega$-integral. We will estimate each of them individually. We begin with $I_{\text {res }}^{b 1}$ and estimate

$$
\begin{aligned}
& \left\|I_{\mathrm{res}}^{b 1}\right\|_{L^{2}\left(\mathbb{S}^{2}\right)}^{2} \\
& \lesssim \sum_{m} \int_{0}^{\pi}\left|\sum_{\ell \geq|m|} \int_{\omega_{-} m-\frac{1}{a m}}^{\omega_{-} m+\frac{1}{a m}}\right| \mathcal{F}_{\mathcal{H}}\left[\psi_{0}^{n}\right] S_{m \ell}\left(a \omega_{-} m\right) \partial_{\omega}\left(\mathfrak{t} \tilde{\mathcal{C}}_{L}\right)(\tilde{\xi})|\mathrm{d} \omega|^{2} \sin \theta \mathrm{d} \theta \\
& \lesssim \sum_{m}\left(\sum_{\ell \geq|m|} \int_{0}^{\pi} \int_{\mathbb{R}}\left(1+\Lambda_{m \ell}^{3}\right)\left|\mathcal{F}_{\mathcal{H}}\left[\psi_{0}^{n}\right]\right|^{2}\left|S_{m \ell}\left(a \omega_{-} m\right)\right|^{2} \mathrm{~d} \omega \sin \theta \mathrm{d} \theta\right) \\
& \times\left(\frac{1}{m} \sum_{\ell \geq|m|\left|\tilde{\xi}-a \omega_{-} m\right|<\frac{1}{m}} \frac{\left|\partial_{\omega}\left(\mathfrak{t} u_{\mathcal{C} \mathcal{H}_{L}}\right)(\tilde{\xi})\right|^{2}}{1+\Lambda_{m \ell}^{3}(\tilde{\xi})}\right) \\
& \lesssim \sum_{m}\left(\sum_{\ell \geq|m|} \int_{\mathbb{R}}\left(1+\Lambda_{m \ell}^{3}\right)\left|\mathcal{F}_{\mathcal{H}}\left[\psi_{0}^{n}\right]\right|^{2} \mathrm{~d} \omega\right) \\
& \quad \times\left(\sum_{\ell \geq|m|} \frac{\Lambda_{m \ell}^{2}\left(a \omega_{-} m\right) \log ^{2}\left(\Lambda_{m \ell}\left(a \omega_{-} m\right)\right)}{1+\Lambda_{m \ell}^{3}\left(a \omega_{-} m\right)}\right) \lesssim D
\end{aligned}
$$

Here we have used Lemma 8.4, Lemma 8.5, Lemma 8.6 and the fact that

$$
\Lambda_{\omega_{-}, m \ell}:=\Lambda_{m \ell}\left(a \omega_{-} m\right) \sim \Lambda_{m \ell}(a \xi)
$$

for all $\left|\xi-\omega_{-} m\right|<\frac{1}{m}$ which in turn is a consequence of Lemma 3.2.

We now control the second term $I_{\text {res }}^{b 2}$ and estimate

$$
\begin{aligned}
& \left\|I_{\mathrm{res}}^{b 2}\right\|_{L^{2}\left(\mathbb{S}^{2}\right)}^{2} \\
& \qquad \sum_{m} \int_{0}^{\pi}\left|\sum_{\ell \geq|m|} \int_{\omega_{-} m-\frac{1}{a m}}^{\omega_{-} m+\frac{1}{a m}}\right| \mathcal{F}_{\mathcal{H}}\left[\psi_{0}^{n}\right] \frac{S_{m \ell}(a \omega)-S_{m \ell}(a \omega-m)}{\omega-\omega_{-} m} \mathfrak{t} \tilde{\mathcal{C}}_{L}|\mathrm{~d} \omega|^{2} \\
& \quad \times \sin \theta \mathrm{d} \theta \\
& \lesssim \sum_{m}\left(\sum_{\ell \geq|m|} \int_{\mathbb{R}}\left(1+\Lambda_{m \ell}^{3}\right)\left|\mathcal{F}_{\mathcal{H}}\left[\psi_{0}^{n}\right]\right|^{2} \mathrm{~d} \omega\right)
\end{aligned}
$$




$$
\begin{aligned}
& \times\left(\sum_{\ell \geq|m|} \int_{\omega_{-} m-\frac{1}{a m}}^{\omega_{-} m+\frac{1}{a m}} \frac{\left|\mathfrak{t} u \tilde{\mathcal{H}}_{L}\right|^{2}}{1+\Lambda_{m \ell}^{3}} \int_{0}^{\pi}\left|\frac{S_{m \ell}(a \omega)-S_{m \ell}\left(a \omega \_m\right)}{\omega-\omega_{-} m}\right|^{2} \sin \theta \mathrm{d} \theta \mathrm{d} \omega\right) \\
\lesssim & \sum_{m}\left(\sum_{\ell \geq|m|} \int_{\mathbb{R}}\left(1+\Lambda_{m \ell}^{3}\right)\left|\mathcal{F}_{\mathcal{H}}\left[\psi_{0}^{n}\right]\right|^{2} \mathrm{~d} \omega\right) \\
& \times\left(\sum_{\ell \geq|m|} \frac{\Lambda_{\omega_{-}, m \ell}}{1+\Lambda_{\omega_{-}, m \ell}^{3}} \int_{\omega_{-} m-\frac{1}{a m}}^{\omega_{-} m+\frac{1}{a m}} \sup _{\left|\tilde{\xi}-\omega_{-} m\right| \leq \frac{1}{m}} \int_{0}^{\pi}\left|\partial_{\omega} S_{m \ell}\right|^{2}(a \tilde{\xi}) \sin \theta \mathrm{d} \theta \mathrm{d} \omega\right) \\
\lesssim & D,
\end{aligned}
$$

where we have used the mean value property for Fréchet derivatives, Lemma 8.4, Lemma 8.5 and Proposition 3.3.

Now, we proceed with $I_{\text {res }}^{a}$, i.e. the first term in (9.13). We begin by recalling the definition of $\mathcal{F}_{\mathcal{H}}\left[\psi_{0}^{n}\right]$ :

$\mathcal{F}_{\mathcal{H}}\left[\psi_{0}^{n}\right]=\frac{\sqrt{r_{+}^{2}+a^{2}}}{\sqrt{2 \pi}} \int_{\mathbb{R}} \int_{\mathbb{S}^{2}} e^{i \omega v} \psi_{0}^{n}\left(v, \theta, \tilde{\phi}_{+}\right) S_{m \ell}(a \omega, \cos \theta) e^{-i m \tilde{\phi}_{+}} \mathrm{d} v \mathrm{~d} \sigma_{\mathbb{S}^{2}}$.

Similar to Lemma 9.2 we will replace the $S_{m \ell}(a \omega)$ appearing in (9.17) with $S_{m \ell}\left(a \omega_{-} m\right)$. In order to do so, we introduce

$$
\begin{aligned}
\hat{I}_{\text {res }}^{a}:= & \frac{1}{\sqrt{2 \pi\left(r_{n}^{2}+a^{2}\right)}} \sum_{m \ell} \text { p.v. } \int_{\omega_{-} m-\frac{1}{a m}}^{\omega_{-} m+\frac{1}{a m}} \frac{e^{-2 i\left(\omega-\omega_{-} m\right) r_{n}^{*}} e^{i \omega u_{0}} \tilde{\mathcal{F}}_{\mathcal{H}}\left[\psi_{0}^{n}\right]}{\omega-\omega_{-} m} \mathrm{~d} \omega \\
& \times e^{i m \phi_{-}^{*}} S_{m \ell}\left(a m \omega_{-}, \cos \theta\right) \mathfrak{t}\left(\omega_{-} m, m, \ell\right) \tilde{\mathcal{C}}_{L}{ }^{\omega_{-}} \\
\tilde{\mathcal{F}}_{\mathcal{H}}\left[\psi_{0}^{n}\right]:= & \frac{\sqrt{r_{+}^{2}+a^{2}}}{\sqrt{2 \pi}} \int_{\mathbb{R}} \psi_{0 m \ell}^{n}(v) e^{i \omega v} \mathrm{~d} v=\sqrt{r_{+}^{2}+a^{2}} \mathfrak{F}\left[\psi_{0 m \ell}^{n}\right],
\end{aligned}
$$

and $^{5}$

$$
\psi_{0 m \ell}^{n}(v):=\int_{\mathbb{S}^{2}} \psi_{0}^{n}\left(v, \theta, \tilde{\phi}_{+}\right) S_{m \ell}\left(a \omega_{-} m, \cos \theta\right) e^{-i m \tilde{\phi}_{+}} \mathrm{d} \sigma_{\mathbb{S}^{2}} .
$$

\section{Lemma 9.3}

$$
\left\|\hat{I}_{\text {res }}^{a}-I_{\text {res }}^{a}\right\|_{L^{2}\left(\mathbb{S}^{2}\right)}^{2} \lesssim D
$$

$\overline{5 \text { Recall that } \mathfrak{F} \text { denotes the standard Fourier transform } \mathfrak{F}[f](\xi)}:=\frac{1}{\sqrt{2 \pi}} \int_{\mathbb{R}} f(x) e^{i \xi x} \mathrm{~d} x$. 
Proof Similarly to the proof of Lemma 9.2, we write

$$
S_{m \ell}(a \omega)=S_{m \ell}\left(a \omega_{-} m\right)+\left(\omega-\omega_{-} m\right) \frac{S_{m \ell}(a \omega)-S_{m \ell}\left(a \omega_{-} m\right)}{\omega-\omega_{-} m} .
$$

for frequencies $\left|\omega-\omega_{-} m\right| \leq \frac{1}{a m}$ in (9.17). Then, using a CauchySchwarz inequality on the sphere, $\sup _{\left|\xi-a \omega_{-} m\right| \leq \frac{1}{m}}\left|\partial_{\xi} \Lambda_{m \ell}(\xi)\right| \lesssim|m|$, $\sup _{\left|\xi-a \omega_{-} m\right| \leq \frac{1}{m}}\left|\partial_{\xi} P(\xi)\right| \lesssim|m|$ (see (3.42)), Proposition 3.3 as well as elliptic estimates, we control the error term as

$$
\begin{aligned}
& \left(1+m^{2} \Lambda_{\omega_{-}, m \ell}^{2}\right)\left|\frac{\mathcal{F}_{\mathcal{H}}-\tilde{\mathcal{F}}_{\mathcal{H}}}{\omega-\omega_{-} m}\right|^{2} \lesssim m\left[\int_{\mathbb{S}^{2}}\left|\int_{\mathbb{R}} e^{i \omega v} \psi_{0}^{n}\left(v, \theta, \tilde{\phi}_{+}\right) \mathrm{d} v\right|^{2} \mathrm{~d} \sigma_{\mathbb{S}^{2}}\right. \\
& \left.+\int_{\mathbb{S}^{2}}\left|\int_{\mathbb{R}} e^{i \omega v} \not^{3} \psi_{0}^{n}\left(v, \theta, \tilde{\phi}_{+}\right) \mathrm{d} v\right|^{2} \mathrm{~d} \sigma_{\mathbb{S}^{2}}\right] .
\end{aligned}
$$

Now, from Lemma 8.4 and Lemma 8.5 we conclude after an application of the Cauchy-Schwarz inequality and Plancherel's theorem that

$$
\left\|\hat{I}_{\text {res }}^{a}-I_{\text {res }}^{a}\right\|_{L^{2}\left(\mathbb{S}^{2}\right)}^{2} \lesssim D
$$

Note that the function $\omega \mapsto \mathfrak{F}\left[\psi_{0 m \ell}^{n}\right](\omega)$ is a $L^{2}\left(\mathbb{Z}_{m} \times \mathbb{Z}_{\ell \geq|m|}\right)$-valued Schwartz function since $v \mapsto \psi_{0 m \ell}^{n}(v)$ is a $L^{2}\left(\mathbb{Z}_{m} \times \mathbb{Z}_{\ell \geq|m|}\right)$-valued Schwartz function. We also define

$$
\begin{aligned}
\tilde{I}_{\text {res }}^{a}:= & \frac{1}{\sqrt{2 \pi\left(r_{n}^{2}+a^{2}\right)}} \sum_{m \ell} \text { p.v. } \int_{\mathbb{R}} \frac{e^{-2 i\left(\omega-\omega_{-} m\right) r_{n}^{*}} e^{i \omega u_{0}} \tilde{\mathcal{F}}_{\mathcal{H}}\left[\psi_{0}^{n}\right]}{\omega-\omega_{-} m} \mathrm{~d} \omega \\
& \times e^{i m \phi_{-}^{*}} S_{m \ell}\left(a m \omega_{-}, \cos \theta\right) \mathfrak{t}\left(\omega_{-} m, m, \ell\right) \tilde{\mathcal{C}}_{\tilde{\mathcal{H}}_{L}}{ }^{\omega_{-}} .
\end{aligned}
$$

Lemma 9.4 We have $\left\|\hat{I}_{\text {res }}^{a}-\tilde{I}_{\text {res }}^{a}\right\|_{L^{2}\left(\mathbb{S}^{2}\right)}^{2} \lesssim D$ for all $r_{n}^{*} \geq r_{0}^{*}$

Proof We use that the spheroidal harmonics $S_{m \ell}\left(a m \omega_{-}, \cos \theta\right) e^{i m \phi_{-}^{*}}$ form an orthonormal basis of $L^{2}\left(\mathbb{S}^{2}\right)$ to estimate 


$$
\begin{aligned}
& \left\|\tilde{I}_{\text {res }}^{a}-\hat{I}_{\text {res }}^{a}\right\|_{L^{2}\left(\mathbb{S}^{2}\right)}^{2} \\
& \quad \lesssim \sum_{m \ell}|m|^{2}\left|\left(\int_{-\infty}^{\omega-m-\frac{1}{a m}}+\int_{\omega_{-} m+\frac{1}{a m}}^{+\infty}\right)\right| \tilde{\mathcal{F}_{\mathcal{H}}}\left[\psi_{0}^{n}\right]\left[\psi_{0}^{n}\right]|\mathrm{d} \omega|{\tilde{\mathcal{C H}_{L}}}^{\omega_{-}} \mathfrak{t}^{\omega_{-}}||^{2} \\
& \left.\quad \lesssim \sum_{m \ell}|m|^{2} \Lambda_{m \ell}\left(a \omega_{-} m\right)\left|\int_{\mathbb{R}}\right| \tilde{\mathcal{F}}_{\mathcal{H}}\left[\psi_{0}^{n}\right]|\mathrm{d} \omega|\right|^{2} \lesssim D
\end{aligned}
$$

where we used the Cauchy-Schwarz inequality in the last step.

Now, we turn to $\tilde{I}_{\text {res }}^{a}$ as defined in (9.25) and first only consider the $\omega$-integral

$$
\operatorname{Int}_{\mathrm{res}}^{a}:=\frac{1}{\sqrt{2 \pi\left(r_{n}^{2}+a^{2}\right)}} \mathrm{p} . \mathrm{v} \cdot \int_{\mathbb{R}} \frac{e^{-2 i\left(\omega-\omega_{-} m\right) r_{n}^{*}} e^{i \omega u_{0}} \tilde{\mathcal{F}_{\mathcal{H}}}\left[\psi_{0}^{n}\right]}{\omega-\omega_{-} m} \mathrm{~d} \omega
$$

We have

$$
\begin{aligned}
\operatorname{Int}_{\mathrm{res}}^{a} & =\frac{\sqrt{r_{+}^{2}+a^{2}}}{\sqrt{r_{n}^{2}+a^{2}}} \frac{1}{\sqrt{2 \pi}} \mathrm{p} \cdot \mathrm{v} \cdot \int_{\mathbb{R}} \frac{\mathfrak{F}\left[\psi_{0 m \ell}^{n}\left(\cdot-u_{0}+2 r_{n}^{*}\right) e^{i \omega_{-} m \cdot}\right]}{\omega} e^{2 i \omega_{-} m r_{n}^{*}} \mathrm{~d} \omega \\
& =\frac{\sqrt{r_{+}^{2}+a^{2}}}{\sqrt{r_{n}^{2}+a^{2}}} \frac{1}{\sqrt{2 \pi}} e^{2 i \omega_{-} m r_{n}^{*}} \mathrm{p} \cdot \mathrm{v} \cdot\left(\frac{1}{\omega}\right)\left[\mathfrak{F}\left[\psi_{0 m \ell}^{n}\left(\cdot-u_{0}+2 r_{n}^{*}\right) e^{i \omega_{-} m \cdot}\right]\right] \\
& =\frac{\sqrt{r_{+}^{2}+a^{2}}}{\sqrt{r_{n}^{2}+a^{2}}} \frac{1}{\sqrt{2 \pi}} e^{2 i \omega_{-} m r_{n}^{*}} i \pi \operatorname{sgn}\left[\psi_{0 m \ell}^{n}\left(\cdot-u_{0}+2 r_{n}^{*}\right) e^{i \omega_{-} m \cdot}\right],
\end{aligned}
$$

where sgn has to be understood as a Schwartz distribution. We have used that $\mathfrak{F}\left[\right.$ p.v. $\left.\left(\frac{1}{\omega}\right)\right]=i \pi \operatorname{sgn}$ in the sense of distributions. Now, since $\psi_{0}$ is smooth, the function $v \mapsto \psi_{0 m \ell}^{n}$ is a Schwartz function with values in the space of superpolynomially decaying sequences in $m, \ell$ as a subspace of $L^{2}\left(\mathbb{Z}_{m} \times\right.$ $\mathbb{Z}_{\ell \geq|m|}$ ). Particularly, this implies that

$$
\mathfrak{t}^{\omega_{-}} \boldsymbol{u}_{\tilde{\mathcal{C H}}_{L}}{ }^{\omega}{ }_{-} \operatorname{Int}_{\text {res }}^{a} \in L^{2}\left(\mathbb{Z}_{m} \times \mathbb{Z}_{\ell \geq|m|} ; L^{\infty}\left(r_{0}^{*}, \infty\right)\right)
$$

so we can project $\tilde{I}_{\text {res }}^{a}$ on $e^{i m \phi_{-}^{*}} S_{m \ell}\left(a m \omega_{-}, \cos \theta\right)$. Indeed, this yields

$$
\begin{aligned}
& \left\langle e^{i m \tilde{\phi}_{-}^{*}} S_{m \ell}\left(a m \omega_{-}, \cos \theta\right), \tilde{I}_{\text {res }}^{a}\right\rangle_{L^{2}\left(\mathbb{S}^{2}\right)} \\
& =\frac{\sqrt{r_{+}^{2}+a^{2}}}{\sqrt{r_{n}^{2}+a^{2}}} \frac{\mathfrak{t}^{\omega_{-}} \tilde{\mathcal{C H}}_{L}^{\omega_{-}}}{\sqrt{2 \pi}} e^{2 i \omega_{-} m r_{n}^{*}} i \pi \operatorname{sgn}\left[\psi_{0 m \ell}^{n}\left(\cdot-u_{0}+2 r_{n}^{*}\right) e^{i \omega_{-} m \cdot}\right] .
\end{aligned}
$$


To summarize, we have decomposed $I$ as

$$
\begin{aligned}
I= & I_{\text {res }}+I_{\text {non-res }}=I_{\text {res }}+I_{\text {non-res }}=\tilde{I}_{\text {res }}^{a}+\left(I_{\text {res }}^{a}-\hat{I}_{\text {res }}^{a}\right) \\
& +\left(\hat{I}_{\text {res }}^{a}-\tilde{I}_{\text {res }}^{a}\right)+I_{\text {res }}^{b}+I_{\text {non-res }},
\end{aligned}
$$

where $\tilde{I}_{\text {res }}^{a}$ satisfies (9.30) and

$$
\left\|\left(I_{\text {res }}^{a}-\hat{I}_{\text {res }}^{a}\right)+\left(\hat{I}_{\text {res }}^{a}-\tilde{I}_{\text {res }}^{a}\right)+I_{\text {res }}^{b}+I_{\text {non-res }}\right\|_{L^{2}\left(\mathbb{S}^{2}\right)} \lesssim D^{\frac{1}{2}} .
$$

Completely analogous to the analysis before, we also decompose $I I$ as

$$
\begin{aligned}
I I & =I I_{\text {res }}+I I_{\text {non-res }}=I I_{\text {res }}+I I_{\text {non-res }} \\
& =\tilde{I I}_{\text {res }}^{a}+\left(I I_{\text {res }}^{a}-\hat{I I} I_{\text {res }}^{a}\right)+\left(\hat{I I} I_{\text {res }}^{a}-\tilde{I I}_{\text {res }}^{a}\right)+I I_{\text {res }}^{b}+I I_{\text {non-res }}
\end{aligned}
$$

where

$$
\left\|\left(I I_{\text {res }}^{a}-\hat{I I}_{\text {res }}^{a}\right)+\left(\hat{I}_{\text {res }}^{a}-\tilde{I I}_{\text {res }}^{a}\right)+I I_{\text {res }}^{b}+I I_{\text {non-res }}\right\|_{L^{2}\left(\mathbb{S}^{2}\right)} \lesssim D^{\frac{1}{2}}
$$

and $\tilde{I I}_{\text {res }}^{a}$ satisfies

$$
\begin{aligned}
& \left\langle e^{i m \phi_{-}^{*}} S_{m \ell}\left(a m \omega_{-}, \cos \theta\right), \tilde{I I}_{\text {res }}^{a}\right\rangle_{L^{2}\left(\mathbb{S}^{2}\right)} \\
& =\frac{\sqrt{r_{+}^{2}+a^{2}}}{\sqrt{r_{n}^{2}+a^{2}}} \frac{\mathfrak{r}^{\omega_{-}} u_{\tilde{\mathcal{C}}_{R}}^{\omega_{-}}}{\sqrt{2 \pi}} i \pi \operatorname{sgn}\left[\psi_{0 m \ell}^{n}\left(\cdot-u_{0}\right) e^{i \omega_{-} m \cdot}\right] .
\end{aligned}
$$

Hence, using

$$
\mathfrak{r}^{\omega_{-}}=-\mathfrak{t}^{\omega_{-}} \text {and } u \tilde{\mathcal{C H}}_{R}{ }^{\omega_{-}}=u \tilde{\mathcal{C}}_{L}{ }^{\omega_{-}},
$$

we obtain

$$
\begin{aligned}
& \left\langle e^{i m \phi_{-}^{*}} S_{m \ell}\left(a m \omega_{-}, \cos \theta\right), \tilde{I}_{\mathrm{res}}^{a}+\tilde{I I}_{\mathrm{res}}^{a}\right\rangle_{L^{2}\left(\mathbb{S}^{2}\right)} \\
& \quad=-i \pi \frac{\sqrt{r_{+}^{2}+a^{2}}}{\sqrt{r_{n}^{2}+a^{2}}} \frac{\mathfrak{r}^{\omega_{-}} u_{\tilde{\mathcal{H}}_{L}}^{\omega_{-}}}{\sqrt{2 \pi}} e^{i \omega_{-} m u_{0}} \int_{-u_{0}}^{2 r_{n}^{*}-u_{0}} \psi_{0 m \ell}^{n}(v) e^{i \omega_{-} m v} \mathrm{~d} v .
\end{aligned}
$$


Now, by construction of $\psi_{0}^{n}$, we have that $\psi_{0 m \ell}^{n}(v)=0$ for $v \geq 2 r_{n}^{*}-u_{0}+\tilde{c}$, where $\tilde{c}$ is a constant only depending on the black hole parameters. In particular, this implies that

$$
\sum_{m \ell}\left|i \pi \frac{\sqrt{r_{+}^{2}+a^{2}}}{\sqrt{r_{n}^{2}+a^{2}}} \frac{\mathrm{r}^{\omega_{-}}-u_{\mathcal{C}_{\mathcal{H}}}{ }^{\omega_{-}}}{\sqrt{2 \pi}} e^{i \omega_{-} m u_{0}}\left(\int_{-\infty}^{-u_{0}}+\int_{2 r_{n}^{*}-u_{0}}^{+\infty}\right) \psi_{0 m \ell}^{n}(v) e^{i \omega_{-} m v} \mathrm{~d} v\right|^{2} \lesssim_{0} D
$$

which allows us to- up to a term bounded by $D^{\frac{1}{2}}$-replace the integral in (9.37) with an integral on the whole real line $v \in \mathbb{R}$. Finally, from Proposition 7.1 (more precisely (7.48)), we obtain

$$
\begin{aligned}
\|\psi\|_{L^{2}\left(\mathbb{S}^{2}\right)}^{2}\left(u_{0}, r_{n}^{*}\right) & =\sum_{m \ell}\left|\pi \frac{\sqrt{r_{+}^{2}+a^{2}}}{\sqrt{r_{n}^{2}+a^{2}}} \frac{\mathfrak{r}^{\omega_{-}}-\tilde{\mathcal{C}}_{\mathcal{\mathcal { H }}_{L}}{ }^{\omega_{-}}}{\sqrt{2 \pi}} \int_{\mathbb{R}} \psi_{0 m \ell}^{n}(v) e^{i \omega_{-} m v} \mathrm{~d} v\right|^{2}+\operatorname{Err}(D) \\
& =\sum_{m \ell}\left|\pi \frac{\mathfrak{r}_{-}^{\omega_{-}} u_{\mathcal{C}_{L}}{ }^{\omega_{-}}}{\sqrt{r_{n}^{2}+a^{2}}} a_{\mathcal{H}}^{R_{n}}\left(\omega=\omega_{-} m\right)\right|^{2}+\operatorname{Err}(D),
\end{aligned}
$$

where $|\operatorname{Err}(D)| \lesssim u_{0} D$ uniformly for all $r_{n}^{*} \geq r_{0}^{*}$. We have established formula (9.6) which concludes the proof of Proposition 9.1.

We will now finish off the proof of Theorem 1. From Lemma 7.3 we have that $a_{\mathcal{H}}^{R_{n}} \rightarrow a_{\mathcal{H}}$ pointwise for fixed $\omega, m, \ell$ as $R_{n} \rightarrow \infty$. We also have the pointwise limit

$$
u \tilde{\mathcal{C H}}_{L}{ }^{\omega-} \rightarrow 1 \text { as } r_{n}^{*} \rightarrow \infty .
$$

Hence, applying Fatou's lemma to (9.39) yields

$$
\liminf _{r_{n}^{*} \rightarrow \infty}\|\psi\|_{L^{2}\left(\mathbb{S}^{2}\right)}^{2}\left(u_{0}, r_{n}^{*}\right) \geq \frac{\pi^{2}}{r_{-}^{2}+a^{2}} \sum_{m \ell}\left|\mathfrak{r}^{\omega_{-}}\right|^{2}\left|a_{\mathcal{H}}\left(\omega=\omega_{-} m, m, \ell\right)\right|^{2}-C_{u_{0}} D,
$$

where $C_{u_{0}}>0$ is a constant depending on $u_{0}$. Since

$$
\left|\mathfrak{r}^{\omega_{-}}\right| \gtrsim|m|
$$

for all $m$ sufficiently large as shown in Lemma 8.7, we obtain

$$
\begin{aligned}
& \liminf _{r_{n}^{*} \rightarrow \infty}\|\psi\|_{L^{2}\left(\mathbb{S}^{2}\right)}^{2}\left(u_{0}, r_{n}^{*}\right) \\
& \quad \gtrsim \frac{\pi^{2}}{r_{-}^{2}+a^{2}} \sum_{i \in \mathbb{N}}\left|m_{i}\right|^{2}\left|a_{\mathcal{H}}\left(\omega=\omega_{-} m_{i}, m_{i}, \ell_{i}\right)\right|^{2}-C_{u_{0}} D .
\end{aligned}
$$


Finally, from Lemma 6.2 we have that

$$
\left|a_{\mathcal{H}}\left(\omega=\omega_{-} m_{i}, m_{i}, \ell_{i}\right)\right| \gtrsim e^{\frac{1}{2} \sqrt{m_{i}}}
$$

for infinitely many $m_{i}$ such that we conclude

$$
\lim _{r_{n}^{*} \rightarrow \infty}\|\psi\|_{L^{2}\left(\mathbb{S}^{2}\right)}^{2}\left(u_{0}, r_{n}^{*}\right)=+\infty .
$$

Since the sequence $r_{n}^{*} \rightarrow \infty$ was arbitrary we obtain (9.1). This concludes the proof of Theorem 1 .

Proof of Corollary 1 Let $\tilde{\Psi}_{0}, \tilde{\Psi}_{1}$ be initial data as in the statement of Corollary 1 and let $\tilde{\psi}$ the arising solution to (1.2). In view of the fact that different azimuthal modes $m$ are $L^{2}\left(\mathbb{S}^{2}\right)$-orthogonal in evolution, it suffices to show the blow-up for the modes $m=m_{i}$, where $m_{i}$ is the sequence in (6.2) associated to the non-Diophantine condition (6.3). Now, the proof of Theorem 1 also carries over for the initial data $\tilde{\Psi}_{0}, \tilde{\Psi}_{1}$ and in particularly the analog of (9.43) holds true. Recalling the definition of $a_{\mathcal{H}}$ in Definition 6.3, the analog of (9.43) for $\tilde{\psi}$ is

$$
\begin{aligned}
& \liminf _{r_{n}^{*} \rightarrow \infty}\|\tilde{\psi}\|_{L^{2}\left(\mathbb{S}^{2}\right)}^{2}\left(u_{0}, r_{n}^{*}\right) \gtrsim \frac{\pi^{2}}{r_{-}^{2}+a^{2}} \sum_{i \in \mathbb{N}} \frac{\left|m_{i}\right|^{2}\left|G\left(\tilde{\Psi}_{0}, \tilde{\Psi}_{1}, m_{i}, \ell_{i}\right)\right|^{2}}{2 \pi\left|\mathfrak{W}\left[u_{\mathcal{H}^{+}}, u_{\infty}\right]\left(\omega=\omega-m_{i}, m_{i}, \ell_{i}\right)\right|^{2}} \\
& -C_{u_{0}} D\left(\tilde{\Psi}_{0}, \tilde{\Psi}_{1}\right) \\
& \left.\gtrsim \sum_{i \in \mathbb{N}}\left|m_{i} e^{\sqrt{\left|m_{i}\right|} \mid}\right| G\left(\tilde{\Psi}_{0}, \tilde{\Psi}_{1}, m_{i}, \ell_{i}\right)\right|^{2}-C_{u_{0}} D\left(\tilde{\Psi}_{0}, \tilde{\Psi}_{1}\right)
\end{aligned}
$$

in view of the non-Diophantine condition $\left|\mathfrak{W}\left[u_{\mathcal{H}^{+}}, u_{\infty}\right]\left(\omega=\omega_{-} m_{i}, m_{i}, \ell_{i}\right)\right|<$ $e^{-\sqrt{m_{i}}}$ as in (6.3). Thus, if the data $\tilde{\Psi}_{0}, \tilde{\Psi}_{1}$ satisfy the genericity condition (9.2), then $\lim _{r \rightarrow r_{-}}\left\|\tilde{\psi}\left(u_{0}, r\right)\right\|_{L^{2}\left(\mathbb{S}^{2}\right)}^{2}=+\infty$ for every $u_{0} \in \mathbb{R}$.

Acknowledgements The author would like to express his gratitude to his advisor Mihalis Dafermos for his support and many valuable comments on the manuscript. The author also thanks Harvey Reall, Igor Rodnianski, Yakov Shlapentokh-Rothman and Claude Warnick for insightful discussions and helpful remarks. The author is also grateful to two anonymous referees for several helpful comments and remarks to improve the manuscript. This work was supported by the EPSRC grant EP/L016516/1, by Dr. Max Rössler, the Walter Haefner Foundation and the ETH Zürich Foundation.. The author thanks Princeton University for hosting him as a VSRC.

Open Access This article is licensed under a Creative Commons Attribution 4.0 International License, which permits use, sharing, adaptation, distribution and reproduction in any medium or format, as long as you give appropriate credit to the original author(s) and the source, provide a link to the Creative Commons licence, and indicate if changes were made. The images or other third party material in this article are included in the article's Creative Commons licence, unless indicated otherwise in a credit line to the material. If material is not included in the article's Creative Commons licence and your intended use is not permitted by statutory regulation or 
exceeds the permitted use, you will need to obtain permission directly from the copyright holder. To view a copy of this licence, visit http://creativecommons.org/licenses/by/4.0/.

\section{A Appendix}

\section{A.1 Airy functions}

We recall the definition of the Airy functions of first and second kind Ai and Bi as follows.

Definition A.1 For $x \in \mathbb{R}$, we define $\operatorname{Ai}(x)$ and $\operatorname{Bi}(x)$ via the improper Riemann integrals

$$
\begin{aligned}
& \operatorname{Ai}(x):=\frac{1}{\pi} \int_{0}^{\infty} \cos \left(\frac{t^{3}}{3}+x t\right) \mathrm{d} t \\
& \operatorname{Bi}(x):=\frac{1}{\pi} \int_{0}^{\infty}\left[\exp \left(-\frac{t^{3}}{3}+x t\right)+\sin \left(\frac{t^{3}}{3}+x t\right)\right] \mathrm{d} t .
\end{aligned}
$$

Equivalently, the Airy functions are the unique solutions of

$$
u^{\prime \prime}=x u
$$

with

$$
\begin{aligned}
& \operatorname{Ai}(0)=\frac{1}{3^{\frac{2}{3}} \Gamma\left(\frac{2}{3}\right)}, \operatorname{Ai}^{\prime}(0)=\frac{-1}{3^{\frac{1}{3}} \Gamma\left(\frac{1}{3}\right)}, \\
& \operatorname{Bi}(0)=\frac{1}{3^{\frac{1}{6}} \Gamma\left(\frac{2}{3}\right)}, \operatorname{Bi}^{\prime}(0)=\frac{3^{\frac{1}{6}}}{\Gamma\left(\frac{1}{3}\right)}
\end{aligned}
$$

such that $\mathfrak{W}_{x}(\operatorname{Ai}(x), \operatorname{Bi}(x))=\frac{1}{\pi}$. Further, we define the constant $c$ as the largest negative root of $\operatorname{Ai}(x)=\operatorname{Bi}(x)$. Then, we introduce the error-control functions

$$
E_{\mathrm{Ai}}(x):=\left\{\begin{array}{ll}
(\operatorname{Bi}(x) / \mathrm{Ai}(x))^{\frac{1}{2}} & x \geq c \\
1 & x \leq c
\end{array} \text { and } M_{\mathrm{Ai}}(x):= \begin{cases}(2 \mathrm{Ai}(x) \operatorname{Bi}(x))^{\frac{1}{2}} & x \geq c \\
\left(\mathrm{Ai}^{2}(x)+\mathrm{Bi}^{2}(x)\right)^{\frac{1}{2}} & x \leq c\end{cases}\right.
$$

and $E_{\mathrm{Ai}}^{-1}(x):=\frac{1}{E_{\mathrm{Ai}}(x)}$. From [94, Chapter 11, §2] we remark that $E_{\mathrm{Ai}}$ is a monotonically increasing function of $x$ which is never less than 1 and moreover,

$$
|\mathrm{Ai}(x)| \leq \frac{M_{\mathrm{Ai}}(x)}{E_{\mathrm{Ai}}(x)} \text { as well as }|\mathrm{Bi}(x)| \leq M_{\mathrm{Ai}}(x) E_{\mathrm{Ai}}(x) .
$$


Furthermore, we have (see [94, Chapter 11, §2] )

$$
\left|M_{\mathrm{Ai}}(x)\right| \lesssim \frac{1}{\langle x\rangle^{\frac{1}{4}}}
$$

for $x \in \mathbb{R}$. Similarly, we define

$$
N_{\mathrm{Ai}}(x):= \begin{cases}\left(\frac{\operatorname{Ai}^{\prime}(x)^{2} \mathrm{Bi}^{\prime}(x)^{2}+\mathrm{Bi}^{\prime}(x)^{2} \mathrm{Ai}(x)^{2}}{\operatorname{Ai}(x) \operatorname{Bi}(x)}\right)^{\frac{1}{2}} & x \geq c \\ \left(\operatorname{Ai}^{\prime}(x)^{2}+\operatorname{Bi}^{\prime}(x)^{2}\right)^{\frac{1}{2}} & x \leq c,\end{cases}
$$

which satisfies (see [94, Chapter 11, §2])

$$
\left|N_{\mathrm{Ai}}(x)\right| \lesssim\langle x\rangle^{\frac{1}{4}}
$$

The Airy functions obey the following asymptotics.

Lemma A.1 ([94, Chapter $11, \S 1, \S 2],[32, \S 9.7])$ For large $x>0$, the asymptotic behaviors of the Airy functions are

$$
\begin{aligned}
\mathrm{Ai}(-x) & =\frac{1}{\sqrt{\pi} x^{\frac{1}{4}}} \cos \left(\frac{2}{3} x^{\frac{3}{2}}-\frac{\pi}{4}\right)+\tilde{\epsilon}_{\mathrm{Ai}}(x), \quad \mathrm{Ai}^{\prime}(-x) \\
& =\frac{x^{\frac{1}{4}}}{\sqrt{\pi}} \sin \left(\frac{2}{3} x^{\frac{3}{2}}-\frac{1}{4} \pi\right)+\tilde{\epsilon}_{\mathrm{Ai}^{\prime}}(x), \\
\mathrm{Bi}(-x) & =\frac{-1}{\sqrt{\pi} x^{\frac{1}{4}}} \sin \left(\frac{2}{3} x^{\frac{3}{2}}-\frac{\pi}{4}\right)+\tilde{\epsilon}_{\mathrm{Ai}}(x), \quad \mathrm{Bi}^{\prime}(-x) \\
& =\frac{x^{\frac{1}{4}}}{\sqrt{\pi}} \cos \left(\frac{2}{3} x^{\frac{3}{2}}-\frac{1}{4} \pi\right)+\tilde{\epsilon}_{\mathrm{Ai}^{\prime}}(x),
\end{aligned}
$$

where $\left|\tilde{\epsilon}_{\mathrm{Ai}}\right| \lesssim x^{-\frac{7}{4}}$ and $\left|\tilde{\epsilon}_{\mathrm{Ai}^{\prime}}\right| \lesssim x^{-\frac{5}{4}}$. In particular, we have

$$
|\mathrm{Ai}(-x)|,|\mathrm{Bi}(-x)| \lesssim \frac{1}{1+x^{\frac{1}{4}}} \text { and }\left|\mathrm{Ai}^{\prime}(-x)\right|,\left|\mathrm{Bi}^{\prime}(-x)\right| \lesssim 1+x^{\frac{1}{4}}
$$

for $x \geq 0$. Moreover, for $x>0$ we have

$$
\begin{aligned}
& 0 \leq \operatorname{Ai}(x) \leq \frac{e^{-\frac{2}{3} x^{\frac{3}{2}}}}{2 \sqrt{\pi} x^{\frac{1}{4}}}, \\
& \left|\operatorname{Ai}^{\prime}(x)\right| \leq \frac{x^{\frac{1}{4}} e^{-\frac{2}{3} x^{\frac{3}{2}}}}{2 \sqrt{\pi}}\left(1+\frac{7}{48 x^{\frac{3}{2}}}\right),
\end{aligned}
$$




$$
\begin{aligned}
& 0 \leq \operatorname{Bi}(x) \leq \frac{e^{\frac{2}{3} x^{\frac{3}{2}}}}{\sqrt{\pi} x^{\frac{1}{4}}}\left(1+\left(\chi_{\mathrm{Ai}}\left(\frac{7}{6}\right)+1\right) \frac{5}{48 x^{\frac{3}{2}}}\right), \\
& 0 \leq \operatorname{Bi}^{\prime}(x) \leq \frac{x^{\frac{1}{4}} e^{\frac{2}{3} x^{\frac{3}{2}}}}{\sqrt{\pi}}\left(1+\left(\frac{\pi}{2}+1\right) \frac{7}{48 x^{\frac{3}{2}}}\right)
\end{aligned}
$$

where $\chi_{\mathrm{Ai}}(x)=\sqrt{\pi} \frac{\Gamma\left(\frac{1}{2} x+1\right)}{\Gamma\left(\frac{1}{2} x+\frac{1}{2}\right)}$.

\section{A.2 Parabolic cylinder functions}

We define the parabolic cylinder functions $U$ and $\bar{U}$ in the following. We refer to [93, Sect. 5] or [32, Chapter 12] for more details.

Definition A.2 For $b \leq 0$ and $x \geq 0$ we define the parabolic cylinder functions

$$
\begin{aligned}
U(b, x)= & \frac{\pi^{\frac{1}{2}} 2^{-\frac{1}{4}(2 b+1)} e^{-\frac{1}{4} x^{2}}}{\Gamma\left(\frac{3}{4}+\frac{1}{2} b\right)}{ }_{1} F_{1}\left(\frac{1}{2} b+\frac{1}{4} ; \frac{1}{2} ; \frac{1}{2} x^{2}\right) \\
& -\frac{\pi^{\frac{1}{2}} 2^{-\frac{1}{4}(2 b-1)}}{\Gamma\left(\frac{1}{4}+\frac{1}{2} b\right)} e^{-\frac{1}{4} x^{2}} x_{1} F_{1}\left(\frac{1}{2} b+\frac{3}{4} ; \frac{3}{2} ; \frac{1}{2} x^{2}\right), \\
\bar{U}(b, x)= & \pi^{-\frac{1}{2}} 2^{-\frac{1}{4}(2 b+1)} \Gamma\left(\frac{1}{4}-\frac{1}{2} b\right) \sin \left(\frac{3}{4} \pi\right. \\
& \left.-\frac{1}{2} b \pi\right) e^{-\frac{1}{4} x^{2}}{ }_{1} F_{1}\left(\frac{1}{2} b+\frac{1}{4} ; \frac{1}{2} ; \frac{1}{2} x^{2}\right) \\
& -\pi^{-\frac{1}{2}} 2^{-\frac{1}{4}(2 b-1)} \Gamma\left(\frac{3}{4}-\frac{1}{2} b\right) \sin \left(\frac{5}{4} \pi\right. \\
& \left.-\frac{1}{2} b \pi\right) e^{-\frac{1}{4} x^{2}} x_{1} F_{1}\left(\frac{1}{2} b+\frac{3}{4} ; \frac{3}{2} ; \frac{1}{2} x^{2}\right),
\end{aligned}
$$

where ${ }_{1} F_{1}(a ; b ; z):=\sum_{n=0}^{\infty} \frac{a^{(n)} z^{n}}{b^{(n)} n !}$ denotes the confluent hypergeometric function. Here, we use the notation $a^{(n)}:=a(a+1)(a+2) \cdots(a+n)$ for the rising factorial.

Remark that $\mathfrak{W}(U, \bar{U})=\sqrt{\frac{2}{\pi}} \Gamma\left(\frac{1}{2}-b\right)$ and that $U$ and $\bar{U}$ solve the equation

$$
-u^{\prime \prime}+\left(\frac{1}{4} x^{2}+b\right) u=0
$$


Moreover, we have (e.g. [93, Sect. 5.2]) that

$$
\begin{aligned}
& U(b, 0)=\pi^{-\frac{1}{2}} 2^{-\frac{1}{4}(2 b+1)} \Gamma\left(\frac{1}{4}-\frac{1}{2} b\right) \sin \left(\frac{1}{4} \pi-\frac{1}{2} b \pi\right), \\
& U^{\prime}(b, 0)=-\pi^{-\frac{1}{2}} 2^{-\frac{1}{4}(2 b-1)} \Gamma\left(\frac{3}{4}-\frac{1}{2} b\right) \sin \left(\frac{3}{4} \pi-\frac{1}{2} b \pi\right), \\
& \bar{U}(b, 0)=\pi^{-\frac{1}{2}} 2^{-\frac{1}{4}(2 b+1)} \Gamma\left(\frac{1}{4}-\frac{1}{2} b\right) \sin \left(\frac{3}{4} \pi-\frac{1}{2} b \pi\right), \\
& \bar{U}^{\prime}(b, 0)=-\pi^{-\frac{1}{2}} 2^{-\frac{1}{4}(2 b-1)} \Gamma\left(\frac{3}{4}-\frac{1}{2} b\right) \sin \left(\frac{5}{4} \pi-\frac{1}{2} b \pi\right) .
\end{aligned}
$$

We define auxiliary functions to control error terms in terms of parabolic cylinder functions. We first define $\rho(b)$ as the largest real root of the equation $\bar{U}(b, x)=U(b, x)$. Note that $\rho(b) \geq 0$ for $b \leq 0$.

Definition A.3 For $b \leq 0$, we set

$$
E_{U}(b, x)= \begin{cases}1 & \text { for } 0 \leq x \leq \rho(b) \\ \sqrt{\frac{\bar{U}(b, x)}{U(b, x)}} & \text { for } x \geq \rho(b)\end{cases}
$$

For fixed $b$, the function $E_{U}(b, x)$ is continuous and non-decreasing in $0 \leq$ $x<\infty$. We denote $E_{U}^{-1}:=\frac{1}{E_{U}}$.

Definition A.4 For $b \leq 0, x \geq 0$, we also define functions $M_{U}$ and $N_{U}$ by

$$
\begin{aligned}
& M_{U}(b, x):= \begin{cases}\sqrt{U^{2}+\bar{U}^{2}} & \text { for } 0 \leq x \leq \rho(b) \\
\sqrt{2 U \bar{U}} & \text { for } \rho(b) \leq x .\end{cases} \\
& N_{U}(b, x):= \begin{cases}\sqrt{U^{\prime 2}+\bar{U}^{\prime 2}} & \text { for } 0 \leq x \leq \rho(b) \\
\sqrt{\frac{U^{\prime 2} \bar{U}^{2}+\bar{U}^{\prime 2} U^{2}}{U \bar{U}}} & \text { for } \rho(b) \leq x .\end{cases}
\end{aligned}
$$

Definition A.5 We define the function $\zeta_{U}$ as

$$
\zeta_{U}(t):= \begin{cases}-\left(\frac{3}{2} \int_{t}^{1}\left(1-\tau^{2}\right)^{\frac{1}{2}} \mathrm{~d} \tau\right)^{\frac{2}{3}} & \text { for } 0 \leq t \leq 1, \\ \left(\frac{3}{2} \int_{1}^{t}\left(\tau^{2}-1\right)^{\frac{1}{2}} \mathrm{~d} \tau\right)^{\frac{2}{3}} & \text { for } t \geq 1 .\end{cases}
$$

Note that we have (see e.g. $[93, \S 5.8]$ )

$$
\begin{aligned}
& |U| \leq E^{-1} M_{U},|\bar{U}| \leq E M_{U} \quad \text { and } \quad|U \bar{U}| \leq M_{U}^{2} \\
& \left|U^{\prime}\right| \leq E^{-1} N_{U},\left|\bar{U}^{\prime}\right| \leq E N_{U} \quad \text { and } \quad|U \bar{U}| \leq N_{U}^{2}
\end{aligned}
$$


for $x \geq 0$ and $b \leq 0$.

Proposition A.1 The envelope function $M_{U}$ satisfies

$$
M_{U}^{2}\left(-\frac{1}{2} \mu^{2}, \mu y \sqrt{2}\right) \lesssim \frac{1}{\mu^{\frac{1}{3}}} \frac{1}{1+\left|\zeta_{U}(y)\right|^{\frac{1}{4}}} \frac{1}{1+\mu^{\frac{2}{3}}\left|\zeta_{U}(y)\right|^{\frac{1}{2}}} \Gamma\left(\frac{1}{2}+\frac{1}{2} \mu^{2}\right)
$$

uniformly in $\mu \geq 1$ and $y \geq 0$, and

$$
M_{U}^{2}\left(-\frac{1}{2} \mu^{2}, \mu y \sqrt{2}\right) \lesssim \frac{1}{1+\sqrt{\mu y}} \Gamma\left(\frac{1}{2}+\frac{1}{2} \mu^{2}\right)
$$

uniformly in $0 \leq \mu \leq 1$ and $y \geq 0$. In particular, $M_{U}$ satisfies

$$
\left|M_{U}\left(-\frac{1}{2} \mu^{2}, \mu y \sqrt{2}\right)\right|^{2} \lesssim \Gamma\left(\frac{1}{2}+\frac{1}{2} \mu^{2}\right)
$$

Proof These estimates follow from [93, Equation (5.23), (6.12) and Sect. 6.2].

\section{References}

1. Arnold, V.I.: Small denominators and problems of stability of motion in classical and celestial mechanics. Uspehi Mat. Nauk 18.6 (114), 91-192 (1963)

2. Baire, R.: Sur les fonctions de variables réelles. Annali di Mat. (3) 3, 1-123 (1899)

3. Baldi, P., Berti, M., Haus, E., Montalto, R.: Time quasi-periodic gravity water waves in finite depth. Invent. Math. 214(2), 739-911 (2018)

4. Benomio, G.: The Stable Trapping Phenomenon for Black Strings and Black Rings and its Obstructions on the Decay of Linear Waves. arXiv:1809.07795, accepted at Anal. PDE (2020)

5. Berti, M.: KAM theory for partial differential equations. Anal. Theory Appl. 35(3), 235267 (2019)

6. Berti, M., Bolle, P.: Sobolev quasi-periodic solutions of multidimensional wave equations with a multiplicative potential. Nonlinearity 25(9), 2579-2613 (2012)

7. Berti, M., Bolle, P.: Quasi-Periodic Solutions of Nonlinear Wave Equations on the dDimensional Torus. EMS Monographs in Mathematics. European Mathematical Society (EMS), Zürich (2020)

8. Besicovitch, A.S.: Sets of fractional dimensions (IV): on rational approximation to real numbers. J. Lond. Math. Soc. 9(2), 126-131 (1934)

9. Bhattacharjee, S., Kumar, S., Sarkar, S.: Mass inflation and strong cosmic censorship in a nonextreme BTZ black hole. Phys. Rev. D 102(4), 044030, 11 (2020)

10. Bhattacharjee, S., Sarkar, S., Virmani, A.: Internal structure of charged AdS black holes. Phys. Rev. D 93(12), 124029 (2016)

11. Bizon, P., Rostworowski, A.: Weakly turbulent instability of anti-de sitter spacetime. Phys. Rev. Lett. 107, 031102 (2011)

12. Breitenlohner, P., Freedman, D.Z.: Stability in gauged extended supergravity. Ann. Phys. 144(2), 249-281 (1982) 
13. Carter, B.: Hamilton-Jacobi and Schrödinger separable solutions of Einstein's equations. Commun. Math. Phys. 10, 280-310 (1968)

14. Christodoulou, D.: On the global initial value problem and the issue of singularities. Class. Quantum Gravity 16(12A), A23-A35 (1999)

15. Christodoulou, D.: The instability of naked singularities in the gravitational collapse of a scalar field. Ann. Math. (2) 149.1, 183-217 (1999)

16. Christodoulou, D.: The formation of black holes in general relativity. EMS Monographs in Mathematics. European Mathematical Society (EMS), Zürich (2009)

17. Christodoulou, D., Klainerman, S.: The Global Nonlinear Stability of the Minkowski Space. Vol. 41 of Princeton Mathematical Series. Princeton University Press (1993)

18. Costa, J. L., Franzen, A. T.: Bounded energy waves on the black hole interior of ReissnerNordström-de Sitter. Ann. Henri Poincaré 18.10 (2017)

19. Costa, J. L., Girão, P. M., Natário, J., Silva, J. D.: On the global uniqueness for the Einstein-Maxwell-scalar field system with a cosmological constant. Part 2. Structure of the solutions and stability of the Cauchy horizon. Commun. Math. Phys. 339(3), 903-947 (2015)

20. Costa, J. L., Girão, P. M., Natário, J., Silva, J. D.: On the global uniqueness for the EinsteinMaxwell-Scalar field system with a cosmological constant: Part 3. Mass inflation and extendibility of the solutions. Ann. PDE 3(1), Art. 8, 55 (2017)

21. Dafermos, M.: Stability and instability of the Cauchy horizon for the spherically symmetric Einstein-Maxwell-scalar field equations. Ann. Math. (2) 158(3), 875-928 (2003)

22. Dafermos, M.: Black holes without spacelike singularities. Commun. Math. Phys. 332(2), 729-757 (2014)

23. Dafermos, M., Holzegel, G.: Dynamic instability of solitons in $4+1$-dimensional gravity with negative cosmological constant. https://www.dpmms.cam.ac.uk/ md384/ ADSinstability.pdf (2006)

24. Dafermos, M., Luk, J.: The interior of dynamical vacuum black holes I: The C0-stability of the Kerr Cauchy horizon (2017). arXiv: 1710.01722

25. Dafermos, M., Rodnianski, I.: The red-shift effect and radiation decay on black hole spacetimes. Commun. Pure Appl. Math. 62(7), 859-919 (2009)

26. Dafermos, M., Shlapentokh-Rothman, Y.: Time-translation invariance of scattering maps and blue-shift instabilities on Kerr black hole spacetimes. Commun. Math. Phys. 350(3), 985-1016 (2017)

27. Dafermos, M., Shlapentokh-Rothman, Y.: Rough initial data and the strength of the blueshift instability on cosmological black holes with $\Lambda>0$. Class. Quantum Grav. 35(19), $195010(2018)$

28. Dias, O. J. C., Eperon, F. C., Reall, H. S., Santos, J. E.: Strong cosmic censorship in de Sitter space. Phys. Rev. D 97.10, 104060, 13 (2018)

29. Dias, O. J. C., Reall, H. S., Santos, J. E.: Strong cosmic censorship: taking the rough with the smooth. J. High Energy Phys. 10 (2018)

30. Dias, O. J. C., Reall, H. S., Santos, J. E.: Strong cosmic censorship for charged de Sitter black holes with a charged scalar field. Class. Quantum Gravity 36.4, 045005, 24 (2019)

31. Dias, O. J., Reall, H. S., Santos, J. E.: The BTZ black hole violates strong cosmic censorship. J. High Energy Phys. 12 (2019)

32. NIST Digital Library of Mathematical Functions. http://dlmf.nist.gov/, Release 1.1.1 of 2021-03-15. F. W. J. Olver, A. B. Olde Daalhuis, D. W. Lozier, B. I. Schneider, R. F. Boisvert, C. W. Clark, B. R. Miller, B. V. Saunders, H. S. Cohl, and M. A. McClain, (eds)

33. Dold, D.: Unstable mode solutions to the Klein-Gordon equation in Kerr-anti-de Sitter spacetimes. Commun. Math. Phys. 350(2), 639-697 (2017)

34. Ellis, G.F.R., MacCallum, M.A.H.: A class of homogeneous cosmological models. Commun. Math. Phys. 12, 108-141 (1969) 
35. Erdogan, M. B., Shakan, G.: Fractal solutions of dispersive partial differential equations on the torus. Selecta Math. (N.S.) 25.1, Paper No. 11, 26 (2019)

36. Falconer, K.: Fractal geometry. Third. Mathematical foundations and applications. John Wiley \& Sons, Ltd., Chichester, pp. xxx+368 (2014)

37. Feckan, M.: Periodic solutions of certain abstract wave equations. Proc. Amer. Math. Soc. 123(2), 465-470 (1995)

38. Fedoryuk, M. V.: Asymptotic analysis. Linear ordinary differential equations, Translated from the Russian by Andrew Rodick. Springer-Verlag, Berlin, pp. viii+363 (1993)

39. Fournodavlos, G., Sbierski, J.: Generic Blow-Up Results for the Wave Equation in the Interior of a Schwarzschild Black Hole. Arch. Ration. Mech. Anal. 235(2), 927-971 (2020)

40. Fournodavlos, G., Smulevici, J.: On the initial boundary value problem for the Einstein vacuum equations in the maximal gauge (2019). arXiv:1912.07338

41. Franzen, A.T.: Boundedness of massless scalar waves on Reissner-Nordström interior backgrounds. Comm. Math. Phys. 343(2), 601-650 (2016)

42. Franzen, A.T.: Boundedness of Massless Scalar Waves on Kerr Interior Backgrounds. Ann. Henri Poincaré 21(4), 1045-1111 (2020)

43. Friedrich, H.: On the existence of n-geodesically complete or future complete solutions of Einstein's field equations with smooth asymptotic structure. Commun. Math. Phys 107.4 (1986)

44. Friedrich, H.: Einstein equations and conformal structure: Existence of anti-de Sitter-type space-times. J. Geom. Phys. 17(2), 125-184 (1995)

45. Gajic, D.: Linear waves in the interior of extremal black holes I. Comm. Math. Phys. 353(2), 717-770 (2017)

46. Gajic, D.: Linear waves in the interior of extremal black holes II. Ann. Henri Poincaré 18(12), 4005-4081 (2017)

47. Gajic, D., Warnick, C.: Quasinormal modes in extremal Reissner-Nordström spacetimes (2021). arXiv: 1910.08479

48. Gannot, O.: Existence of quasinormal modes for Kerr-AdS black holes. Ann. Henri Poincaré 18(8), 2757-2788 (2017)

49. Gannot, O., Wrochna, M.: Propagation of singularities on AdS spacetimes for general boundary conditions and the holographic Hadamard condition. J. Inst. Math. Jussieu, pp. $1-61(2020)$

50. Gramchev, T., Yoshino, M.: WKB analysis to global solvability and hypoellipticity. Publ. Res. Inst. Math. Sci. 31(3), 443-464 (1995)

51. Harman, G.: Metric number theory. Vol. 18. London Mathematical Society Monographs. New Series. The Clarendon Press, Oxford University Press, New York, pp. xviii+297 (1998)

52. Hawking, S. W., Reall, H. S.: Charged and rotating AdS black holes and their CFT duals. Phys. Rev. D (3) 61.2, pp. 024014, 10 (2000)

53. Hewitt, C.G., Wainwright, J.: A dynamical systems approach to Bianchi cosmologies: orthogonal models of class B. Classical Quantum Gravity 10(1), 99-124 (1993)

54. Hintz, P.: Boundedness and decay of scalar waves at the Cauchy horizon of the Kerr spacetime. Comment. Math. Helv. 92(4), 801-837 (2017)

55. Hintz, P.: A sharp version of Price's law for wave decay on asymptotically flat spacetimes. arXiv preprint arXiv:2004.01664 (2020)

56. Hintz, P., Vasy, A.: Analysis of linear waves near the Cauchy horizon of cosmological black holes. J. Math. Phys. 58.8, pp. 081509, 45 (2017)

57. Hintz, P., Vasy, A.: The global non-linear stability of the Kerr-de Sitter family of black holes. Acta Math. 220(1), 1-206 (2018)

58. Hollands, S., Klein, C., Zahn, J.: Quantum stress tensor at the Cauchy horizon of the Reissner-Nordström-de Sitter spacetime. Phys. Rev. D 102.8, pp. 085004, 10 (2020) 
59. Hollands, S., Wald, R.M., Zahn, J.: Quantum instability of the Cauchy horizon in ReissnerNordström-de Sitter spacetime. Classical and Quantum Gravity 37(11), 115009 (2020)

60. Holzegel, G.: On the massive wave equation on slowly rotating Kerr-AdS spacetimes. Comm. Math. Phys. 294(1), 169-197 (2010)

61. Holzegel, G.: Well-posedness for the massive wave equation on asymptotically anti-de Sitter spacetimes. J. Hyperbolic Differ. Equ. 9(2), 239-261 (2012)

62. Holzegel, G., Luk, J., Smulevici, J., Warnick, C.: Asymptotic Properties of Linear Field Equations in Anti-de Sitter Space. Comm. Math. Phys. 374(2), 1125-1178 (2020)

63. Holzegel, G., Smulevici, J.: Decay properties of Klein-Gordon fields on Kerr-AdS spacetimes. Comm. Pure Appl. Math. 66(11), 1751-1802 (2013)

64. Holzegel, G., Smulevici, J.: Stability of Schwarzschild-AdS for the spherically symmetric Einstein-Klein-Gordon system. Comm. Math. Phys. 317(1), 205-251 (2013)

65. Holzegel, G., Smulevici, J.: Quasimodes and a lower bound on the uniform energy decay rate for Kerr-AdS spacetimes. Anal. PDE 7(5), 1057-1090 (2014)

66. Holzegel, G., Warnick, C.M.: Boundedness and growth for the massive wave equation on asymptotically anti-de Sitter black holes. J. Funct. Anal. 266(4), 2436-2485 (2014)

67. Jarník, V.: Über die simultanen diophantischen Approximationen. Math. Z. 33(1), 505$543(1931)$

68. Kapitanski, L., Rodnianski, I.: Does a quantum particle know the time? Emerging applications of number theory (Minneapolis, MN, Vol. 109. IMA Vol. Math. Appl. Springer, New York 1999, 355-371 (1996)

69. Kehle, C.: Diophantine Approximation as Cosmic Censor for AdS Black Holes. PhD thesis. University of Cambridge (2020)

70. Kehle, C.: Uniform Boundedness and Continuity at the Cauchy Horizon for Linear Waves on Reissner-Nordström-AdS Black Holes. Commun. Math. Phys. 376(1), 145-200 (2020)

71. Kehle, C., de Moortel, M. V.: Strong Cosmic Censorship in the presence of matter: the decisive effect of horizon oscillations on the black hole interior geometry (2021). arXiv:2105.04604

72. Kehle, C., Shlapentokh-Rothman, Y.: A Scattering Theory for Linear Waves on the Interior of Reissner-Nordström Black Holes. Ann. Henri Poincaré 20(5), 1583-1650 (2019)

73. Khintchine, A.: Zur metrischen Theorie der diophantischen Approximationen. Math. Z. 24(1), 706-714 (1926)

74. Kolmogorov, A. N.: On conservation of conditionally periodic motions for a small change in Hamilton's function. Dokl. Akad. Nauk SSSR (N.S.) 98, pp. 527-530 (1954)

75. Kristensen, S.: Diophantine approximation and the solubility of the Schrödinger equation. Phys. Lett. A 314(1-2), 15-18 (2003)

76. Landau, L. D., Lifshitz, E. M.: Quantum mechanics: non-relativistic theory. Course of Theoretical Physics, Vol. 3. Addison-Wesley Series in Advanced Physics. Translated from Russian. Pergamon Press Ltd., pp. xii+515 (1958)

77. Lapidus, M. L., van Frankenhuijsen, M.: eds. Fractal geometry and applications: a jubilee of Benoit Mandelbrot. Part 1. Vol. 72. Proceedings of Symposia in Pure Mathematics. Analysis, number theory, and dynamical systems. American Mathematical Society, Providence, RI, pp. xii+517 (2004)

78. Luk, J., de Moortel, M. V.: Nonlinear interaction of three impulsive gravitational waves I: main result and the geometric estimates (2021). arXiv:2101.08353

79. Luk, J., Oh, S.-J.: Proof of linear instability of the Reissner-Nordström Cauchy horizon under scalar perturbations. Duke Math. J. 166(3), 437-493 (2017)

80. Luk, J., Oh, S.-J.: Strong cosmic censorship in spherical symmetry for two-ended asymptotically flat initial data I. The interior of the black hole region. Ann. of Math. (2) 190.1, pp. 1-111 (2019) 
81. Luk, J., Oh, S.-J.: Strong cosmic censorship in spherical symmetry for two-ended asymptotically flat initial data II: the exterior of the black hole region. Ann. PDE 5.1, Paper No. 6, 194 (2019)

82. Luk, J., Rodnianski, I.: Local propagation of impulsive gravitational waves. Comm. Pure Appl. Math. 68(4), 511-624 (2015)

83. Luk, J., Sbierski, J.: Instability results for the wave equation in the interior of Kerr black holes. J. Funct. Anal. 271(7), 1948-1995 (2016)

84. Moschidis, G.: The Einstein-null dust system in spherical symmetry with an inner mirror: structure of the maximal development and Cauchy stability (2017). arXiv:1704.08685

85. Moschidis, G.: A proof of the instability of AdS for the Einstein-massless Vlasov system (2018). arXiv: 1812.04268

86. Moschidis, G.: The characteristic initial-boundary value problem for the Einstein-massless Vlasov system in spherical symmetry (2018). arXiv:1812.04274

87. Moschidis, G.: A proof of the instability of AdS for the Einstein-null dust system with an inner mirror. Anal. PDE 13(6), 1671-1754 (2020)

88. Moser., J.: On invariant curves of area-preserving mappings of an annulus. Nachr. Akad. Wiss. Göttingen Math.-Phys. Kl. II 1962, pp. 1-20 (1962)

89. Moser, J.: Is the solar system stable? Math. Intelligencer 1(2), 65-71 (1978)

90. Novák, B.: Remark on periodic solutions of a linear wave equation in one dimension. Comment. Math. Univ. Carolinae 15, 513-519 (1974)

91. Olver, F.W.J.: Error bounds for the Liouville-Green (or WKB) approximation. Proc. Cambridge Philos. Soc. 57, 790-810 (1961)

92. Olver, F.W.J.: Error bounds for first approximations in turning-point problems. J. Soc. Indust. Appl. Math. 11, 748-772 (1963)

93. Olver, F. W. J.: Second-order linear differential equations with two turning points. Philos. Trans. Roy. Soc. London Ser. A 278, pp. 137-174 (1975)

94. Olver, F.W.J.: Asymptotics and special functions. AKP Classics. A K Peters Ltd, Wellesley, MA (1997)

95. Ori, A.: Inner structure of a charged black hole: an exact mass-inflation solution. Phys. Rev. Lett. 67(7), 789-792 (1991)

96. Pandya, A., Pretorius, F.: The Rotating Black Hole Interior: Insights from Gravitational Collapse in AdS3. arXiv preprint arXiv:2002.07130 (2020)

97. Penrose, R.: A remarkable property of plane waves in general relativity. Rev. Modern Phys. 37, 215-220 (1965)

98. Penrose, R.: Structure of space-time. Battelle Rencontres., pp. 121-235 (1968)

99. Penrose, R.: Gravitational Collapse. Gravitational Radiation and Gravitational Collapse. Ed. by C. DeWitt-Morette. Vol. 64 of IAU Symposium. Springer, pp. 82-91 (1974)

100. Poisson, E., Israel, W.: Internal structure of black holes. Phys. Rev. D (3) 41.6, pp. 17961809 (1990)

101. Ptashnik, B.I.: Ill-Posed Boundary-Value Problems for Partial Differential Equations (In Russian), p. 264. Naukova Dumka, Kiev (1984)

102. Radermacher, K.: Strong cosmic censorship in orthogonal Bianchi class B perfect fluids and vacuum models. Ann. Henri Poincaré 20(3), 689-796 (2019)

103. Reall, H.: A possible failure of determinism in general relativity. Physics 11, 6 (2018)

104. Ringström, H.: Curvature blow up in Bianchi VIII and IX vacuum spacetimes. Classical Quantum Gravity 17(4), 713-731 (2000)

105. Ringström, H.: The Bianchi IX attractor. Ann. Henri Poincaré 2(3), 405-500 (2001)

106. Ringström, H.: Existence of an asymptotic velocity and implications for the asymptotic behavior in the direction of the singularity in T3-Gowdy. Comm. Pure Appl. Math. 59(7), 977-1041 (2006)

107. Ringström, H.: Strong cosmic censorship in T3-Gowdy spacetimes. Ann. of Math. (2) 170.3, pp. 1181-1240 (2009) 
108. Sbierski, J.: On holonomy singularities in general relativity and the $\mathrm{C} 0 ; 1$ loc inextendibility of spacetimes (2020). arXiv:2007.12049

109. Schwartz, L.: Sur certains espaces de fonctions différentiables à valeurs vectorielles. Séminaire Schwartz 1. Exposé no. 10 (1954)

110. Teschl, G.: Mathematical methods in quantum mechanics. Second. Vol. 157. Graduate Studies in Mathematics. With applications to Schrödinger operators. American Mathematical Society, Providence, RI, pp. xiv+358 (2014)

111. Trèves, F.: Topological vector spaces, distributions and kernels. Academic Press, New York- London, pp. xvi+624 (1967)

112. Van de Moortel, M.: Stability and instability of the sub-extremal Reissner-Nordström black hole interior for the Einstein-Maxwell-Klein-Gordon equations in spherical symmetry. Comm. Math. Phys. 360(1), 103-168 (2018)

113. Van de Moortel, M.: The breakdown of weak null singularities inside black holes (2019). arXiv: 1912.10890

114. Van de Moortel, M.: Mass inflation and the C2-inextendibility of spherically symmetric charged scalar field dynamical black holes. Comm. Math. Phys. 382(2), 1263-1341 (2021)

115. Wainwright, J., Hsu, L.: A dynamical systems approach to Bianchi cosmologies: orthogonal models of class A. Classical Quantum Gravity 6(10), 1409-1431 (1989)

116. Wang, W.-M.: Energy supercritical nonlinear Schrödinger equations: quasiperiodic solutions. Duke Math. J. 165(6), 1129-1192 (2016)

117. Wang, W.-M.: Quasi-periodic solutions for nonlinear wave equations. C. R. Math. Acad. Sci. Paris 353(7), 601-604 (2015)

118. Warnick, C.M.: The massive wave equation in asymptotically AdS spacetimes. Comm. Math. Phys. 321(1), 85-111 (2013)

119. Warnick, C.M.: On quasinormal modes of asymptotically anti-de Sitter black holes. Comm. Math. Phys. 333(2), 959-1035 (2015)

Publisher's Note Springer Nature remains neutral with regard to jurisdictional claims in published maps and institutional affiliations. 